\title{
Nuclear axial current operators to fourth order in chiral effective field theory
}

\author{
H. Krebs, ${ }^{1, *}$ E. Epelbaum, ${ }^{1,2, \dagger}$ and U.-G. Meißner ${ }^{3,4,5, \ddagger}$ \\ ${ }^{1}$ Institut für Theoretische Physik II, Ruhr-Universität Bochum, D-44780 Bochum, Germany \\ ${ }^{2}$ Kavli Institute for Theoretical Physics, University of California, Santa Barbara, CA 93016, USA \\ ${ }^{3}$ Helmholtz-Institut für Strahlen- und Kernphysik and Bethe Center for Theoretical Physics, \\ Universität Bonn, D-53115 Bonn, Germany \\ ${ }^{4}$ Institut für Kernphysik, Institute for Advanced Simulation, \\ and Jülich Center for Hadron Physics, Forschungszentrum Jülich, D-52425 Jülich, Germany \\ ${ }^{5}$ JARA - High Performance Computing, Forschungszentrum Jülich, D-52425 Jülich, Germany
}

(Dated: October 20, 2016)

\begin{abstract}
We present the complete derivation of the nuclear axial charge and current operators as well as the pseudoscalar operators to fourth order in the chiral expansion relative to the dominant onebody contribution using the method of unitary transformation. We demonstrate that the unitary ambiguity in the resulting operators can be eliminated by the requirement of renormalizability and by matching of the pion-pole contributions to the nuclear forces. We give expressions for the renormalized single-, two- and three-nucleon contributions to the charge and current operators and pseudoscalar operators including the relevant relativistic corrections. We also verify explicitly the validity of the continuity equation.
\end{abstract}

PACS numbers: 13.75.Cs,21.30.-x

\section{INTRODUCTION}

The past quarter century has witnessed enormous progress towards formulating low-energy nuclear physics within a systematically improvable and well-founded framework of chiral effective field theory (EFT) as initiated by Weinberg in the early 1990ties [1]. It relies on the most general effective Lagrangian which incorporates the chiral symmetry of QCD and the various patterns of its breaking. For the two-flavor case of the up- and down-quarks, the effective Lagrangian is written in terms of pions, which are identified with (pseudo)-Goldstone bosons of the broken chiral symmetry $\mathrm{SU}(2)_{L} \times \mathrm{SU}(2)_{R} \rightarrow \mathrm{SU}(2)_{V}$, and the relevant matter fields such as the nucleons, complemented by various external sources that parametrize e.g. the explicit chiral symmetry breaking. The Goldstone-boson nature of the pions enables their perturbative treatment at low energy within the framework of chiral perturbation theory. When processes involving two or more nucleons are considered, chiral perturbation theory allows one to derive interaction potentials and exchange current operators to be used in the framework of the $A$-nucleon Schrödinger equation. The formulation thus reduces to the conventional quantum mechanical many-body problem which can be efficiently dealt with numerically using a variety of $a b$ initio approaches developed over the past decades. In addition to being firmly rooted in the symmetries of QCD, the important advantages of nuclear chiral EFT in comparison with more phenomenological approaches comprise its systematic improvability, the consistency between two- and many-body forces and exchange current operators, a unified treatment of processes involving pions and the intimate relation between nucleon and nuclear structure [2].

The chiral EFT approach outlined above and based on pions and nucleons as the only active degrees of freedom has been extensively exploited to derive nuclear forces and to analyze few- and many-nucleon systems, see Refs. [3, 4] for review articles. Recently, the description of the two-nucleon $(2 \mathrm{~N})$ force has been pushed to fifth order in the chiral 
expansion [5, 6], i.e. the simultaneous expansion in powers of the nucleon three-momenta and pion masses, and even most of the sixth-order terms have been derived [7]. Three- (3N), four- (4N) and more-nucleon forces start contributing at third, fourth and sixth orders, respectively. They have been worked out completely up to fourth order in the chiral expansion [8-13], see also Refs. [14-17] for the derivation of some of the fifth-order terms in the three-nucleon force. A systematic investigation of the role of the many-body forces is an important frontier in low-energy nuclear physics [18]. With all these developments, coupled with on-going efforts towards a reliable quantification of various sources of theoretical uncertainties [19-24], nuclear chiral EFT is now entering the precision era [25].

Few- and many-nucleon reactions with external electroweak and pionic probes have also been investigated in the framework of chiral EFT, see e.g. [3, 26, 27] and references therein. In particular, electromagnetic exchange current operators were first discussed in this framework in the seminal paper by Park et al. [28], who, however only focused on the near-threshold kinematics. More recently, the electromagnetic currents were re-derived for more general kinematical conditions by the JLab-Pisa group [29-31] using the framework of time-ordered perturbation theory (TOPT) and by the Bochum-Bonn group [32, 33] within the method of unitary transformation (MUT) [34, 35], which will be described below. It is important to keep in mind that nuclear forces and currents are not directly observable. Contrary to the S-matrix, they are not uniquely defined and can be changed by means of unitary transformations or, equivalently, by changing the basis in the Fock space. It is, therefore, important to maintain consistency between nuclear forces and current operators to ensure that all these objects correspond to the same choice of the basis in the Fock space. Being derived within the same theoretical approach, the exchange electromagnetic charge and current operators worked out in Refs. [32, 33] are, by construction, consistent with the expressions for the nuclear forces given in Refs. [9, 10, 12-16, 36]. Notice further that the unitary ambiguity associated with the freedom in the choice of the basis in the Fock space has been shown in $[10,33]$ to be strongly reduced by demanding renormalizability at the level of the nuclear Hamiltonian and current operators. In particular, exploiting this freedom in a systematic way was crucial to ensure cancellations between the ultraviolet divergences appearing in loop contributions to the one-pion exchange electromagnetic current operator and the corresponding counterterms in the effective Lagrangian leading to finite matrix elements of the currents [33]. Given the different choices for the unitary phases made by the JLab-Pisa and Bochum-Bonn groups, it is not surprising that the resulting expressions for the current operators exhibit strong differences, see also [37] for a discussion. First exploratory applications of (some of) the novel exchange current contributions derived in $[32,33]$ to the ${ }^{2} \mathrm{H}$ and ${ }^{3} \mathrm{He}$ photodisintegration reactions yielded promising results and have shown a significant sensitivity to the new terms in certain observables [38], see also Ref. [39] for a study of the magnetic form factor of the deuteron. A more complete treatment using the last generation of the chiral nucleon-nucleon potentials of Refs. [5, 21] would require the implementation of the regularization scheme consistent with the interactions. Work along these lines is in progress, see Ref. [40] for a first step in this direction.

In this paper we focus on the iso-triplet axial-vector charge and current operators which have been first considered in the framework of chiral EFT in the pioneering paper by Park et al. [41], see also [42], who, however, have ignored pion-pole terms and contributions from reducible-like diagrams involving purely nucleonic states (at least) in one of the time orderings. The resulting expressions have been employed in a number of studies of few-nucleon processes and in nuclear structure calculations, see [43-46] for recent examples. There are several reasons for a strong interest in developing a precision theory for nuclear electroweak reactions in the framework of chiral EFT. First, the lowest-order short-range two-nucleon axial current operator depends on a low-energy constant (LEC) which also contributes to the leading $3 \mathrm{~N}$ force $[9,47]$, the $\mathrm{P}$-wave pion production operator in $2 \mathrm{~N}$ collisions [48, 49] and to pion photoproduction and radiative capture reactions in the $2 \mathrm{~N}$ system [50,51]. Thus, chiral EFT opens an intriguing possibility for "bridging" these very different reactions within a unified theoretical approach [52]. Secondly, there is a strong interest in improving the accuracy and reliability of theoretical predictions for nuclear reactions involving neutrinos such as e.g. the solar proton-proton fusion and the hep processes which figure importantly in nuclear astrophysics. Clearly, this requires a precise determination of the short-range part of the axial current operator. This is the main motivation of the ongoing MuSun experiment at PSI [53] which aims at the determination of the rate of muon capture on the deuteron with a precision of $1.5 \%$. Notice that the theoretical predictions for the doublet capture rate $\Lambda_{1 / 2}$ show a significant spread, see e.g. [54, 55]. Last but not least, the appearance of the same LEC in the leading $3 \mathrm{~N}$ force and the short-range part of the $2 \mathrm{~N}$ weak current opens an exciting possibility to perform nontrivial precision tests of chiral EFT in few-nucleon systems by carrying out a simultaneous calculation of nucleon-deuteron scattering, the binding energy, radius and the precisely known half-life of ${ }^{3} \mathrm{H}$ and the muon capture rates on ${ }^{2} \mathrm{H}$ and ${ }^{3} \mathrm{He}$, accompanied with a careful analysis of the theoretical uncertainties along the lines of Refs. [21, 22].

Recently, the $2 \mathrm{~N}$ contributions to the axial charge and current operators at the leading one-loop order were re-derived by Baroni et al. [56] using TOPT in the same framework as used by the JLab-Pisa group to derive the electromagnetic currents in Refs. [29-31]. However, as already pointed out, the resulting expressions for certain contributions to the 

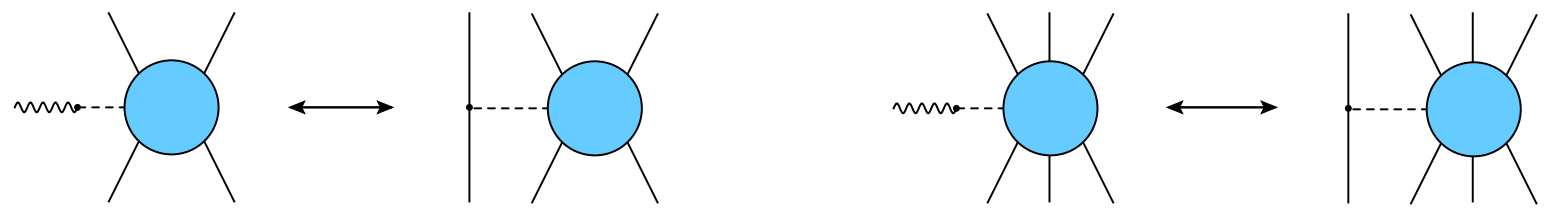

FIG. 1: Matching of the pion-pole contributions to the axial current to the corresponding terms in the nuclear force as explained in the text. Solid, dashed and wavy lines refer to nucleons, pions and the external axial sources, respectively. Solid dots denote the the lowest-order vertices from the effective Lagrangians $\mathcal{L}_{\pi}^{(2)}$ and $\mathcal{L}_{\pi N}^{(1)}$, while shaded circles represent the irreducible parts of the corresponding amplitudes.

electromagnetic currents differ from the ones of Refs. [32, 33] worked out by our group using the MUT. Specifically, while the expressions for the two-pion exchange terms derived by both groups agree with each other, the one-pion exchange and contact operators are different. Notice further that JLab-Pisa group has not performed a complete renormalization of the one-pion exchange current and charge operators. In [37], the origin of some of these differences was clarified. In particular, the one-loop contributions to the short-range current operator re-derived in that work were found to vanish in agreement with our results, while the revised one-loop contributions to the charge operator still turned out to be different from those of our work [33]. These remaining differences can probably be explained by the unitary ambiguity of the considered operators which manifests itself in a different treatment of reducible-like diagrams in the two approaches. Notice that while the unitary ambiguity of the nuclear Hamiltonian, charge and current operators is systematically addressed in our approach by employing a broad class of unitary transformations (UTs) on the nucleonic subspace of the Fock space compatible with the chiral order of the calculation, the JLab-Pisa group has examined only the kind of UTs associated with different off-the-energy-shell extensions of the one-pion exchange potential [31], see also Ref. [57] for a related early work. In this context, it is important to emphasize that the inclusion of a broader class of unitary transformations was found to be necessary to renormalize the one-loop contributions to the $3 \mathrm{~N}$ force $[10,36]$ and to the one-pion exchange current operator [33].

The above discussion provides a strong motivation to derive the exchange axial charge and current operators using the MUT, which was employed in the calculations of nuclear forces in Refs. $[9,10,12-16,36]$. In this paper, we fill this gap and consider the contributions to the nuclear axial charge and current operators to fourth order in the chiral expansion relative to the leading one-body terms, i.e. to leading two-loop, one-loop and tree-level order for the $1 \mathrm{~N}, 2 \mathrm{~N}$ and $3 \mathrm{~N}$ terms, respectively, which are, per construction, consistent with the expressions for the nuclear forces in Refs. $[9,10,12-16,36]$. We find a very high degree of unitary ambiguity in the resulting operators which is parametrized by 33 continuously varying parameters $\alpha_{i}^{a x}$ plus an additional phase $\beta_{1}^{a x}$. Such a richness of the possible UTs can be traced back to the appearance of pion-pole contributions. In addition to the renormalizability requirement, we demand that the pion-pole contributions to the $1 \mathrm{~N}, 2 \mathrm{~N}$ and $3 \mathrm{~N}$ axial current operators match the corresponding expressions for the $2 \mathrm{~N}, 3 \mathrm{~N}$ and $4 \mathrm{~N}$ forces, evaluated at the pion pole, see Fig. 1. This choice is not only the most natural one but is expected to be advantageous in calculations of observables utilizing a finite cutoff. In particular, it will provide a simple way to regularize the pion-pole contributions to the axial current operator in exactly the same way as done in the chiral nuclear potentails. The resulting expressions for the exchange axial charge and current operators turn out to be independent on the phases of the considered UTs. Further, the same approach is applied to derive the nuclear pseudoscalar currents to fourth order in the chiral expansion relative to the dominant one-body contribution. This allows us to perform a highly nontrivial check of our results by explicitly verifying the continuity equation for all considered contributions to the charge and current operators.

Our paper is organized as follows. In section II we discuss in detail the formalism to derive nuclear forces and current operators in chiral EFT using the MUT. In particular, we consider constraints imposed by the chiral symmetry and work out the explicit form of the continuity equations for the iso-triplet vector and axial current operators. We also address the relation of the axial charge and current operators to the $S$-matrix and work out constraints imposed by Poincaré invariance. Finally, we discuss in this section the unitary ambiguity of the resulting operators and specify our standard choice of the unitary phases fixed by the requirement of renormalizability and by matching of the pion-pole contributions to the corresponding nuclear forces. We then specify our notation in section III and derive explicit expressions for one-, two- and three-nucleon axial charge and current operators up to fourth order in the chiral expansion relative to the leading single-nucleon axial current contribution in sections IV, V and VI, respectively. Our final results for the various contributions to the axial charge and current operators are summarized in section VII. 
Next, section VIII is devoted to the derivation of the pseudoscalar current operator, while our final results for the various contributions are summarized in section IX. The validity of the continuity equation for all derived operators is verified in section X. Next, in section XI, we compare our expressions with those obtained by Baroni et al. in Refs. [56, 76]. Finally, the main results of our paper are summarized in section XII. Last but not least, the appendices $\mathrm{A}, \mathrm{B}$ and $\mathrm{C}$ contain a general proof of simultaneous block diagonalizability of the generators of the Poincaré group in the Fock space and explicit definitions of the employed additional unitary transformations.

\section{CHIRAL EFT FOR NUCLEAR FORCES AND CURRENTS: FOUNDATIONS}

Throughout this work, we restrict ourselves to the two-flavor case of the up- and down-quarks and employ the heavybaryon formulation of chiral EFT based on pions and nucleons as the active degrees of freedom. For the purpose of our work, the following terms in the effective Lagrangian describing the interactions between pions and nucleons in the presence of external sources are needed:

$$
\mathcal{L}_{\mathrm{eff}}=\mathcal{L}_{\pi}^{(2)}+\mathcal{L}_{\pi}^{(4)}+\mathcal{L}_{\pi N}^{(1)}+\mathcal{L}_{\pi N}^{(2)}+\mathcal{L}_{\pi N}^{(3)}+\mathcal{L}_{N N}^{(0)}+\mathcal{L}_{\pi N N}^{(1)}
$$

where the superscripts refer to the number of derivatives and/or insertions of the pion mass $M_{\pi}$. Here and in what follows, we employ the operator basis given in Refs. [58] and [59] for the pionic and pion-nucleon Lagrangians $\mathcal{L}_{\pi}$ and $\mathcal{L}_{\pi N}$, respectively. The relevant terms are also listed in appendix A of Ref. [56] (except for vertices involving pseudoscalar sources), where the same effective Lagrangian is used. For the $2 \mathrm{~N}$ Lagrangian $\mathcal{L}_{N N}^{(0)}$, we use the standard notation employed in the calculations of nuclear forces, see e.g. [3]. It involves two terms with the corresponding LECs denoted as $C_{S}$ and $C_{T}$. The Lagrangian $\mathcal{L}_{\pi N N}^{(1)}$ can also be found in [3] and involves just a single term with the corresponding LEC denoted by $D .{ }^{1}$

In the pion and single-nucleon sectors, the effective chiral Lagrangian can be used to calculate the scattering amplitude within the chiral expansion in powers of $Q \in\left\{M_{\pi} / \Lambda_{b}, p / \Lambda_{b}\right\}$, where $p$ refers to four- (three-)momenta of external pions (nucleons) while $\Lambda_{b}$ denotes the breakdown scale which is expected to be of the order of $\Lambda_{b} \equiv \Lambda_{\chi} \sim M_{\rho} \sim$ $4 \pi F_{\pi} \sim 1 \mathrm{GeV}$. As already mentioned in the introduction, in the few-nucleon sector, the perturbative chiral expansion is carried out for the kernel of the corresponding dynamical equation such as e.g. the Lippmann-Schwinger equation for the case of two nucleons or its generalizations for systems involving three- and more nucleons. In the absence of external sources, the kernel is identified with the (interacting part of the) nuclear Hamiltonian while $A$-body terms describing the coupling to the external vector and axial vector sources give rise to the $A$-nucleon electroweak charge and current operators. The breakdown scale of the chiral expansion for nuclear forces was estimated in Ref. [21] to be of the order of $\Lambda_{b} \sim 600 \mathrm{MeV}$. Throughout this work, we adopt the counting scheme for the nucleon mass $m$ which is usually employed in few-nucleon calculations, see $[1,3,60]$ for more details, namely $m \sim \Lambda_{b}^{2} / M_{\pi}$, instead of assigning $m \sim \Lambda_{b}$ as done in the single-baryon sector.

The derivation of the nuclear forces, charge and current operators requires a subtraction of reducible contributions generated by iterations of the dynamical equation. This can be achieved using a variety of approaches including TOPT and the MUT, see [60] for a pedagogical account of various techniques. We now briefly outline the main steps in the derivation within the MUT, see Ref. [33] for a more complete description.

\section{A. Interactions with time derivatives}

As the first step in the derivation of the nuclear forces and currents in chiral EFT using the MUT, we employ the canonical formalism to determine the Hamiltonian for pions, nucleons and external sources from the heavy-baryon effective chiral Lagrangian. Due to the appearance of derivative couplings in the effective Lagrangian, the derivation of the Hamiltonian requires a careful treatment. Even at lowest orders in the chiral expansion, one encounters

\footnotetext{
1 This LEC is usually expressed in terms of a dimensionless constant $c_{D}[9]$.
} 
interactions with time derivatives acting on the pion and nucleon fields. At higher orders, there appear even second or higher-order time derivatives. However, in order to derive the Hamiltonian via a Legendre transformation in the usual way, we need a Lagrangian which includes at most one time derivative of the fields. By using the equations of motion (field redefinitions), one can always eliminate all second- and higher-order time derivatives of the pion fields and all time derivatives of the nucleon fields. The resulting modified Lagrangian represents an equally good starting point for our calculations as the original one since field redefinitions do not affect on-shell scattering amplitudes. With the modified Lagrangian, we can construct the effective Hamiltonian in the usual way. Time derivatives of the nucleon fields do not show up in the interaction terms in the static limit and are always suppressed by inverse powers of the nucleon mass $m$. As an example, consider the following term in the effective Lagrangian ${ }^{2}$

$$
\frac{1}{m^{2}} N^{\dagger}\left(D^{2}-(v \cdot D)^{2}\right) i v \cdot D N+\text { h.c. }
$$

where $N$ denotes the nucleon field, $v$ is the nucleon four-velocity and $D$ is the covariant derivative. We refer the reader to Ref. [59] for more details on the notation and explicit expressions for the pion-nucleon Lagrangian. The above term must be taken into account as it generates a relativistic $1 / \mathrm{m}^{2}$-contribution to the single-nucleon axial vector current $\overrightarrow{\boldsymbol{A}}_{1 \mathrm{~N}}$ at order $Q$. When performing calculations within the MUT, it is convenient to use the equation of motion for the nucleon field

$$
i v \cdot D N=-g_{A} S \cdot u N+\mathcal{O}\left(Q^{2}\right)
$$

or, equivalently, perform a field redefinition

$$
N \rightarrow N-\frac{1}{m^{2}}\left(D^{2}-(v \cdot D)^{2}\right) N
$$

to eliminate the term in Eq. (2.2) in favor of the new vertex

$$
-g_{A} \frac{1}{m^{2}} N^{\dagger}\left(D^{2}-(v \cdot D)^{2}\right) S \cdot u N+\text { h.c. },
$$

which does not involve the time derivative of the nucleon field but a series of higher-order terms which are irrelevant for our present application.

For details concerning the treatment of the $\bar{d}_{22}$-vertex, which involves a time derivative of the external axial vector source, the reader is referred to appendix $\mathrm{C}$.

\section{B. "Strong" unitary transformations}

After the elimination of the various terms involving time derivatives as described in the previous section, the canonical formalism can be applied straightforwardly to derive the effective Hamiltonian $H$ corresponding to the Lagrangian in Eq. (2.1), which governs the pion-nucleon dynamics in the presence of external fields. In general, the chiral EFT Hamiltonian $H[a, v, s, p]$ depends on external axial-vector, vector, scalar and pseudoscalar sources $a_{\mu}, v_{\mu}, s$ and $p$, respectively, whose flavor structure and transformation properties with respect to chiral rotations will be specified in section II D. All these sources are functions of space and time.

Next, one has to integrate out the pion fields, i.e. to decouple the purely nucleonic subspace of the Fock space from the rest. This is achieved via a suitably chosen unitary transformation on the Fock space. Following Okubo [35], the unitary operator $U_{\text {Okubo }}$ can be parametrized in terms of an operator $A=\lambda A \eta$. Here and in what follows, $\eta$ and $\lambda$ denote projection operators onto the purely nucleonic and the remaining parts of the Fock space with the properties $\eta^{2}=\eta, \lambda^{2}=\lambda, \eta \lambda=\lambda \eta=0$ and $\eta+\lambda=1$. The requirement of decoupling of the $\eta$-subspace of the Fock space, $\eta U_{\text {Okubo }}^{\dagger} H_{s} U_{\text {Okubo }} \lambda=\lambda U_{\text {Okubo }}^{\dagger} H_{s} U_{\text {Okubo }} \eta=0$, leads to the nonlinear decoupling equation for the operator $A$,

$$
\lambda\left(H_{s}-\left[A, H_{s}\right]-A H_{s} A\right) \eta=0 .
$$

\footnotetext{
2 Notice that all quantities in the effective chiral Lagrangian are defined in the chiral limit. Our final results are, however, expressed in terms of physical masses and coupling constants.
} 
Here, $H_{s}$ is defined as

$$
H_{s}:=H\left[0,0, s=m_{q}, 0\right],
$$

where $m_{q}$ is the light quark mass, which we will express in terms of the physical pion mass via the relation

$$
2 B m_{q}=M_{\pi}^{2}+\mathcal{O}\left(M_{\pi}^{4}\right) .
$$

From here on, we work in the isospin limit $m_{q}=m_{u}=m_{d}$. The LEC $B$ can be extracted from quark condensate in the isospin limit

$$
\langle 0|\bar{u} u| 0\rangle=\langle 0|\bar{d} d| 0\rangle=-F^{2} B\left(1+\mathcal{O}\left(m_{q}\right)\right),
$$

where $F$ denotes the pion decay constant in the chiral limit. The solution of the decoupling equation and the computation of the operators $U_{\text {Okubo }}$ and $\eta U_{\text {Okubo }}^{\dagger} H U_{\text {Okubo }} \eta$ are carried out perturbatively within the chiral expansion. This is most easily achieved by counting the inverse powers of the hard scale as explained in Ref. [36]. Specifically, the various terms in the interaction part of the Hamiltonian $H_{I}$ can be classified according to the inverse mass dimension $\kappa$ of the corresponding coupling constants,

$$
H_{I}=\sum_{\kappa} H^{(\kappa)} \quad \text { with } \quad \kappa=d+\frac{3}{2} a+b+c-4 .
$$

Here, $d$ is the number of derivatives and/or insertions of $M_{\pi}$ while $a, b$ and $c$ refer to the number of nucleon fields, pion fields and external sources, in order. Notice that we only consider terms with, at most, a single coupling to the axial or pseudoscalar source so that $c=0$ or 1 . Further, one has $\kappa \geq 1$ for interaction terms with $c=0$ and $\kappa \geq-1$ $(\kappa \geq-2)$ for vertices involving a coupling to the axial (pseudoscalar) source, i.e. with $c=1$. It is easy to see [36] that the chiral dimension $\nu$ of the resulting nuclear forces $V$, charge and current operators $\boldsymbol{A}^{0}$ and $\overrightarrow{\boldsymbol{A}}$, which can be read off from the terms in $\eta U_{\text {Okubo }}^{\dagger} H U_{\text {Okubo }} \eta$ involving a coupling to the external axial source $\boldsymbol{a}^{\mu}$, is determined by the overall inverse mass dimension of the coupling constants. Specifically, for the nuclear forces, one has

$$
\nu=-2+\sum_{i} V_{i} \kappa_{i}
$$

while the chiral dimension of the nuclear charge and current operators is given by

$$
\nu=-3+\sum_{i} V_{i} \kappa_{i}
$$

where the shift relative to the dimension of the nuclear forces accounts for the mass dimension of the external source. Here, $V_{i}$ denotes the number of vertices of a type $\kappa_{i}$. Using these results, the decoupling equation can be solved recursively by utilizing an expansion in powers of the inverse overall mass dimension of the coupling constants, and the resulting contributions to $\eta U_{\text {Okubo }}^{\dagger} H U_{\text {Okubo }} \eta$ can be worked out to a desired order $\nu$, see $[12,13,32,33,36,60]$ for more details and explicit expressions. Notice further that while formulating the power counting in terms of the inverse mass dimension $\kappa$ is particularly convenient for algebraic calculations within the MUT, one can also express the chiral dimension $\nu$ in terms of different variables such as the number of loops, see Ref. [1]. This notation is commonly employed in chiral perturbation theory and is particularly convenient when using diagrammatic approaches. We refer the readers to Ref. [60] for further details.

The Okubo parametrization of the UT in terms of the operator $A=\lambda A \eta$ does not describe the most general possible UT. In particular, one can subsequently perform additional UTs on the $\eta$ space. Such additional UTs are, in fact, required to achieve renormalizability of the nuclear forces [10] (and current operators). The additional UTs employed on the $\eta$-space may or may not depend on the external sources. Here and in what follows, we will denote the "strong" $\eta$-space transformations, which do not depend on the external sources, by $U_{\eta}$. Since the corresponding generators, by definition, do not depend on the external sources, the operators $U_{\eta}$ are not explicitly time-dependent.

\section{Unitary transformations involving external sources}

The second class of unitary $\eta$ space transformations are the ones which explicitly depend on external sources. We denote them by $U[a, v, s, p]$. Since these transformations explicitly depend on external sources, they also do explicitly 
depend on time $t$. In the absence of external sources, they, by definition, are required to reduce to:

$$
U\left[0,0, m_{q}, 0\right]=1 .
$$

In general, a time-dependent unitary transformation $U(t)$ of a given Hamiltonian $H$ (which might have an explicit time dependence through external sources) is not just given by $U^{\dagger}(t) H U(t)$. This can be easily seen from the Schrödinger equation

$$
i \frac{\partial}{\partial t} \Psi=H \Psi
$$

that leads to

$$
i \frac{\partial}{\partial t} U(t) U^{\dagger}(t) \Psi=U(t) i \frac{\partial}{\partial t} U^{\dagger}(t) \Psi+\left(i \frac{\partial}{\partial t} U(t)\right) U^{\dagger}(t) \Psi=H U(t) U^{\dagger}(t) \Psi
$$

Multiplying both sides by $U^{\dagger}(t)$ and bringing the term with the time-derivative of the unitary transformation on the right-hand side, we obtain the Schrödinger equation for the transformed state $\Psi^{\prime}=U^{\dagger}(t) \Psi$ in the form

$$
i \frac{\partial}{\partial t} \Psi^{\prime}=\left[U^{\dagger}(t) H U(t)-U^{\dagger}(t)\left(i \frac{\partial}{\partial t} U(t)\right)\right] \Psi^{\prime}
$$

Thus, the unitary transformation of the Hamiltonian $H$ is given by

$$
H \rightarrow U^{\dagger}(t) H U(t)+\left(i \frac{\partial}{\partial t} U^{\dagger}(t)\right) U(t)
$$

We see that in the case of a time-dependent UT, there is an additional term which depends on the time-derivative of the operator $U(t)$. For this reason, the transformed Hamiltonian in our case depends on external sources and their time derivatives:

$$
\begin{aligned}
& H_{\mathrm{eff}}[a, \dot{a}, v, \dot{v}, s, \dot{s}, p, \dot{p}]= \\
& \eta U^{\dagger}[a, v, s, p] U_{\eta}^{\dagger} U_{\text {Okubo }}^{\dagger} H[a, v, s, p] U_{\text {Okubo }} U_{\eta} U[a, v, s, p] \eta+\eta\left(i \frac{\partial}{\partial t} U^{\dagger}[a, v, s, p]\right) U[a, v, s, p] \eta
\end{aligned}
$$

For $a=v=p=0$ and $s=m_{q}$ we obtain the nuclear potential

$$
V:=H_{\mathrm{eff}}\left[a=0, \dot{a}=0, v=0, \dot{v}=0, s=m_{q}, \dot{s}=0, p=0, \dot{p}=0\right]-H_{0},
$$

where $H_{0}$ is the free nucleon Hamiltonian. The individual contributions to $V$ have a form similar to those obtained in TOPT and are given by a sequence of vertices and the corresponding energy denominators. For example, the leading and subleading contributions to the nuclear force $V^{\left(Q^{0}\right)}$ and $V^{\left(Q^{2}\right)}$ constructed solely from the lowest order $\pi N$ coupling proportional to the nucleon axial-vector constant $g_{A}$ from $\mathcal{L}_{\pi N}^{(1)}$ have the form

$$
\begin{aligned}
V^{\left(Q^{0}\right)} & =-\eta H_{2,1}^{(1)} \frac{\lambda^{1}}{E_{\pi}} H_{2,1}^{(1)} \eta \\
V^{\left(Q^{2}\right)} & =-\frac{1}{2} \eta H_{2,1}^{(1)} \frac{\lambda^{1}}{E_{\pi}} H_{2,1}^{(1)} \frac{\lambda^{2}}{E_{\pi}} H_{2,1}^{(1)} \frac{\lambda^{1}}{E_{\pi}} H_{2,1}^{(1)} \eta+\frac{1}{2} \eta H_{2,1}^{(1)} \frac{\lambda^{1}}{E_{\pi}^{2}} H_{2,1}^{(1)} \eta H_{2,1}^{(1)} \frac{\lambda^{1}}{E_{\pi}} H_{2,1}^{(1)} \eta+\text { h.c. }
\end{aligned}
$$

where we have adopted the notation introduced in [36] with $H_{a, b}^{(\kappa)}$ denoting an interaction from the Hamiltonian with $a$ nucleon and $b$ pion fields. Further, $\lambda^{i}$ denotes a projection operator onto states with $i$ pions while $E_{\pi}=$ $\sum_{i} \omega_{i}=\sum_{i} \sqrt{\vec{p}_{i}^{2}+M_{\pi}^{2}}$ is the pion kinetic energy. The operator $V^{\left(Q^{0}\right)}$ contributes to the nucleon self-energy and gives rise to the one-pion exchange $2 \mathrm{~N}$ potential while the terms in $V^{\left(Q^{2}\right)}$ contribute to the nucleon self-energy, renormalization of the one-pion exchange $2 \mathrm{~N}$ potential, the leading two-pion exchange $2 \mathrm{~N}$ potential and the tree-level two-pion exchange $3 \mathrm{~N}$ forces (which, however, turn out to vanish). The explicit form of these contributions is easily obtained by substituting the explicit expressions for the vertices $H_{2,1}^{(1)}$, written in second quantization, and performing the algebra. Here and in what follows, all loop integrals are calculated in the standard way using dimensional regularization. Finally, we emphasize that the contributions which do not involve reducible topologies can be more efficiently calculated using the Feynman graph technique. This is, in fact, the way some of the presented results are obtained. 


\section{Chiral symmetry constraints and the continuity equations}

Under chiral $\mathrm{SU}(2)_{L} \times \mathrm{SU}(2)_{R}$ rotations, the external sources transform as

$$
\begin{aligned}
r_{\mu} & \rightarrow r_{\mu}^{\prime}=R r_{\mu} R^{\dagger}+i R \partial_{\mu} R^{\dagger}, \\
l_{\mu} & \rightarrow l_{\mu}^{\prime}=L l_{\mu} L^{\dagger}+i L \partial_{\mu} L^{\dagger}, \\
s+i p & \rightarrow s^{\prime}+i p^{\prime}=R(s+i p) L^{\dagger}, \\
s-i p & \rightarrow s^{\prime}-i p^{\prime}=L(s-i p) R^{\dagger} .
\end{aligned}
$$

The vector and axial-vector sources can be expressed as a linear combination of the left- and right-handed sources:

$$
v_{\mu}=\frac{1}{2}\left(r_{\mu}+l_{\mu}\right) \quad \text { and } \quad a_{\mu}=\frac{1}{2}\left(r_{\mu}-l_{\mu}\right) .
$$

In the above expressions, $R$ and $L$ denote independent chiral $\mathrm{SU}(2)$ transformations which can be parametrized in the exponential form

$$
R=\exp \left(\frac{i}{2} \boldsymbol{\tau} \cdot \boldsymbol{\epsilon}_{R}(\vec{x}, t)\right) \quad \text { and } \quad L=\exp \left(\frac{i}{2} \boldsymbol{\tau} \cdot \boldsymbol{\epsilon}_{L}(\vec{x}, t)\right)
$$

Using the standard parametrization of the external sources in terms of the isoscalar and isovector components [61],

$$
v_{\mu}=v_{\mu}^{(s)}+\frac{1}{2} \boldsymbol{\tau} \cdot \boldsymbol{v}, \quad a_{\mu}=\frac{1}{2} \boldsymbol{\tau} \cdot \boldsymbol{a}, \quad s=s_{0}+\boldsymbol{\tau} \cdot \boldsymbol{s}, \quad p=p_{0}+\boldsymbol{\tau} \cdot \boldsymbol{p}
$$

the transformation properties of the sources with respect to infinitesimal $\mathrm{SU}(2)_{L} \times \mathrm{SU}(2)_{R}$ rotations have the form

$$
\begin{aligned}
\boldsymbol{v}_{\mu} & \rightarrow \boldsymbol{v}_{\mu}^{\prime}=\boldsymbol{v}_{\mu}+\boldsymbol{v}_{\mu} \times \boldsymbol{\epsilon}_{V}+\boldsymbol{a}_{\mu} \times \boldsymbol{\epsilon}_{A}+\partial_{\mu} \boldsymbol{\epsilon}_{V}, \\
\boldsymbol{a}_{\mu} & \rightarrow \boldsymbol{a}_{\mu}^{\prime}=\boldsymbol{a}_{\mu}+\boldsymbol{a}_{\mu} \times \boldsymbol{\epsilon}_{V}+\boldsymbol{v}_{\mu} \times \boldsymbol{\epsilon}_{A}+\partial_{\mu} \boldsymbol{\epsilon}_{A}, \\
s_{0} & \rightarrow s_{0}^{\prime}=s_{0}-\boldsymbol{p} \cdot \boldsymbol{\epsilon}_{A}, \\
\boldsymbol{s} & \rightarrow \boldsymbol{s}^{\prime}=\boldsymbol{s}+\boldsymbol{s} \times \boldsymbol{\epsilon}_{V}-p_{0} \boldsymbol{\epsilon}_{A}, \\
i p_{0} & \rightarrow i p_{0}^{\prime}=i\left(p_{0}+\boldsymbol{s} \cdot \boldsymbol{\epsilon}_{A}\right), \\
i \boldsymbol{p} & \rightarrow i \boldsymbol{p}^{\prime}=i\left(\boldsymbol{p}+\boldsymbol{p} \times \boldsymbol{\epsilon}_{V}+s_{0} \boldsymbol{\epsilon}_{A}\right),
\end{aligned}
$$

where

$$
\boldsymbol{\epsilon}_{V}=\frac{1}{2}\left(\boldsymbol{\epsilon}_{R}+\boldsymbol{\epsilon}_{L}\right) \quad \text { and } \quad \boldsymbol{\epsilon}_{A}=\frac{1}{2}\left(\boldsymbol{\epsilon}_{R}-\boldsymbol{\epsilon}_{L}\right)
$$

Notice that as it is well known, the singlet axial-vector current is not conserved as the $\mathrm{U}(1)_{A}$ is anomalously broken.

We now proceed similar to Ref. [62]. Starting with the original Schrödinger equation ${ }^{3}$

$$
i \frac{\partial}{\partial t} \Psi=H_{\mathrm{eff}}[a, \dot{a}, v, \dot{v}, s, \dot{s}, p, \dot{p}] \Psi
$$

we expect that there is an (in general, time-dependent) unitary transformation $U$ on the Fock space such that

$$
i \frac{\partial}{\partial t} U^{\dagger} \Psi=H_{\mathrm{eff}}\left[a^{\prime}, \dot{a}^{\prime}, v^{\prime}, \dot{v}^{\prime}, s^{\prime}, \dot{s}^{\prime}, p^{\prime}, \dot{p}^{\prime}\right] U^{\dagger} \Psi
$$

which means that observables are not affected by chiral rotations. In other words, we expect that the Hamiltonians $H_{\mathrm{eff}}\left[a^{\prime}, \dot{a}^{\prime}, v^{\prime}, \dot{v}^{\prime}, s^{\prime}, \dot{s}^{\prime}, p^{\prime}, \dot{p}^{\prime}\right]$ and $H_{\mathrm{eff}}[a, \dot{a}, v, \dot{v}, s, \dot{s}, p, \dot{p}]$ are unitary equivalent:

$$
H_{\mathrm{eff}}\left[a^{\prime}, \dot{a}^{\prime}, v^{\prime}, \dot{v}^{\prime}, s^{\prime}, \dot{s}^{\prime}, p^{\prime}, \dot{p}^{\prime}\right]=U^{\dagger} H_{\mathrm{eff}}[a, \dot{a}, v, \dot{v}, s, \dot{s}, p, \dot{p}] U+\left(i \frac{\partial}{\partial t} U^{\dagger}\right) U
$$

\footnotetext{
${ }^{3}$ In general, also second and higher order time-derivatives of external sources can appear in the Hamiltonian. These terms, however, are only relevant at higher chiral orders beyond the accuracy of the current work.
} 
We make an ansatz for the unitary transformation $U$ by writing it in the form

$$
U=\exp \left(i \int d^{3} x\left[\boldsymbol{R}_{0}^{v}(\vec{x}) \cdot \boldsymbol{\epsilon}_{V}(\vec{x}, t)+\boldsymbol{R}_{1}^{v}(\vec{x}) \cdot \dot{\boldsymbol{\epsilon}}_{V}(\vec{x}, t)+\boldsymbol{R}_{0}^{a}(\vec{x}) \cdot \boldsymbol{\epsilon}_{A}(\vec{x}, t)+\boldsymbol{R}_{1}^{a}(\vec{x}) \cdot \dot{\boldsymbol{\epsilon}}_{A}(\vec{x}, t)\right]\right),
$$

with $R_{0,1}^{v, a}(\vec{x})$ being some Hermitian field operators in the Schrödinger picture. Eq. (2.29) can be used to derive the continuity equation for the currents. Setting $v=\dot{v}=a=\dot{a}=p=\dot{p}=\dot{s}=s=0$ and $s_{0}=m_{q}=\left(m_{u}+m_{d}\right) / 2$ on the right-hand side of Eq. (2.29) and keeping only terms linear in $\boldsymbol{\epsilon}_{V}, \boldsymbol{\epsilon}_{A}$ and their time derivatives, we obtain

$$
\begin{aligned}
& \left.H_{\mathrm{eff}}\left[a^{\prime}, \dot{a}^{\prime}, v^{\prime}, \dot{v}^{\prime}, s^{\prime}, \dot{s}^{\prime}, p^{\prime}, \dot{p}^{\prime}\right]\right|_{v=\dot{v}=a=\dot{a}=p=\dot{p}=\dot{s}=0, s=m_{q}}= \\
& W+\int d^{3} x\left(i\left[W, \boldsymbol{R}_{0}^{v}(\vec{x})\right] \cdot \boldsymbol{\epsilon}_{V}(\vec{x}, t)+i\left[W, \boldsymbol{R}_{1}^{v}(\vec{x})\right] \cdot \dot{\boldsymbol{\epsilon}}_{V}(\vec{x}, t)+i\left[W, \boldsymbol{R}_{0}^{a}(\vec{x})\right] \cdot \boldsymbol{\epsilon}_{A}(\vec{x}, t)\right. \\
& \left.+i\left[W, \boldsymbol{R}_{1}^{a}(\vec{x})\right] \cdot \dot{\boldsymbol{\epsilon}}_{A}(\vec{x}, t)+\boldsymbol{R}_{0}^{v}(\vec{x}) \cdot \dot{\boldsymbol{\epsilon}}_{V}(\vec{x}, t)+\boldsymbol{R}_{1}^{v}(\vec{x}) \cdot \ddot{\boldsymbol{\epsilon}}_{V}(\vec{x}, t)+\boldsymbol{R}_{0}^{a}(\vec{x}) \cdot \dot{\boldsymbol{\epsilon}}_{A}(\vec{x}, t)+\boldsymbol{R}_{1}^{a}(\vec{x}) \cdot \ddot{\boldsymbol{\epsilon}}_{A}(\vec{x}, t)\right),
\end{aligned}
$$

with $W \equiv H_{0}+V$. On the other hand, we can directly expand the left-hand side of Eq. (2.29) in $\boldsymbol{\epsilon}_{V}, \boldsymbol{\epsilon}_{A}$ and their time derivatives to get

$$
\begin{aligned}
& \left.H_{\mathrm{eff}}\left[a^{\prime}, \dot{a}^{\prime}, v^{\prime}, \dot{v}^{\prime}, s^{\prime}, \dot{s}^{\prime}, p^{\prime}, \dot{p}^{\prime}\right]\right|_{v=\dot{v}=a=\dot{a}=p=\dot{p}=\dot{s}=s=0, s_{0}=m_{q}=} \\
& W+\int d^{3} x\left(\boldsymbol{V}_{\mu}^{(0)}(\vec{x}) \cdot \partial^{\mu} \boldsymbol{\epsilon}_{V}(\vec{x}, t)+\boldsymbol{V}_{\mu}^{(1)}(\vec{x}) \cdot \partial^{\mu} \dot{\boldsymbol{\epsilon}}_{V}(\vec{x}, t)+\boldsymbol{A}_{\mu}^{(0)}(\vec{x}) \cdot \partial^{\mu} \boldsymbol{\epsilon}_{A}(\vec{x}, t)+\boldsymbol{A}_{\mu}^{(1)}(\vec{x}) \cdot \partial^{\mu} \dot{\boldsymbol{\epsilon}}_{A}(\vec{x}, t)\right. \\
& \left.+m_{q} \boldsymbol{P}^{(0)}(\vec{x}) \cdot \boldsymbol{\epsilon}_{A}(\vec{x}, t)+m_{q} \boldsymbol{P}^{(1)}(\vec{x}) \cdot \dot{\boldsymbol{\epsilon}}_{A}(\vec{x}, t)\right)
\end{aligned}
$$

where the vector, axial-vector and pseudoscalar currents are defined by

$$
\begin{aligned}
V_{\mu}^{(0) j}(\vec{x}):=\frac{\delta H_{\mathrm{eff}}}{\delta v_{j}^{\mu}(\vec{x}, t)}, \quad V_{\mu}^{(1) j}(\vec{x}):=\frac{\delta H_{\mathrm{eff}}}{\delta \dot{v}_{j}^{\mu}(\vec{x}, t)}, \quad A_{\mu}^{(0) j}(\vec{x}):=\frac{\delta H_{\mathrm{eff}}}{\delta a_{j}^{\mu}(\vec{x}, t)}, \\
A_{\mu}^{(1) j}(\vec{x}):=\frac{\delta H_{\mathrm{eff}}}{\delta \dot{a}_{j}^{\mu}(\vec{x}, t)}, \quad P_{j}^{(0)}(\vec{x}):=\frac{\delta H_{\mathrm{eff}}}{\delta p_{j}(\vec{x}, t)}, \quad P_{j}^{(1)}(\vec{x}):=\frac{\delta H_{\mathrm{eff}}}{\delta \dot{p}_{j}(\vec{x}, t)},
\end{aligned}
$$

with $j=1,2,3$ an isospin index. In all these expressions, the functional derivatives are taken at $v=\dot{v}=a=\dot{a}=p=$ $\dot{p}=\dot{s}=s=0$ and $s_{0}=m_{q}$. Matching Eqs. (2.31) and (2.32) with respect to $\ddot{\boldsymbol{\epsilon}}_{V}(\vec{x}, t)$ and $\ddot{\boldsymbol{\epsilon}}_{A}(\vec{x}, t)$, we read off

$$
\boldsymbol{R}_{1}^{v}(\vec{x})=\boldsymbol{V}_{0}^{(1)}(\vec{x}), \quad \boldsymbol{R}_{1}^{a}(\vec{x})=\boldsymbol{A}_{0}^{(1)}(\vec{x}) .
$$

Next, matching the coefficients in front of the first derivatives $\dot{\boldsymbol{\epsilon}}_{V}(\vec{x}, t)$ and $\dot{\boldsymbol{\epsilon}}_{A}(\vec{x}, t)$ yields

$$
\begin{aligned}
\boldsymbol{R}_{0}^{v}(\vec{x})+i\left[W, \boldsymbol{R}_{1}^{v}(\vec{x})\right] & =\boldsymbol{V}_{0}^{(0)}(\vec{x})-\vec{\nabla} \cdot \overrightarrow{\boldsymbol{V}}^{(1)}(\vec{x}), \\
\boldsymbol{R}_{0}^{a}(\vec{x})+i\left[W, \boldsymbol{R}_{1}^{a}(\vec{x})\right] & =\boldsymbol{A}_{0}^{(0)}(\vec{x})-\vec{\nabla} \cdot \overrightarrow{\boldsymbol{A}}^{(1)}(\vec{x})+m_{q} \boldsymbol{P}^{(1)}(\vec{x}) .
\end{aligned}
$$

Finally, matching the coefficients in front of $\epsilon_{V}(\vec{x}, t)$ and $\epsilon_{A}(\vec{x}, t)$ gives

$$
\begin{aligned}
i\left[W, \boldsymbol{R}_{0}^{v}(\vec{x})\right] & =-\vec{\nabla} \cdot \overrightarrow{\boldsymbol{V}}^{(0)}(\vec{x}), \\
i\left[W, \boldsymbol{R}_{0}^{a}(\vec{x})\right] & =-\vec{\nabla} \cdot \overrightarrow{\boldsymbol{A}}^{(0)}(\vec{x})+m_{q} \boldsymbol{P}^{(0)}(\vec{x}) .
\end{aligned}
$$

Combining these relations, we obtain the continuity equations

$$
\begin{aligned}
i\left[W, \boldsymbol{V}_{0}^{(0)}(\vec{x})-\vec{\nabla} \cdot \overrightarrow{\boldsymbol{V}}^{(1)}(\vec{x})-i\left[W, \boldsymbol{V}_{0}^{(1)}(\vec{x})\right]\right] & =-\vec{\nabla} \cdot \overrightarrow{\boldsymbol{V}}^{(0)}(\vec{x}), \\
i\left[W, \boldsymbol{A}_{0}^{(0)}(\vec{x})-\vec{\nabla} \cdot \overrightarrow{\boldsymbol{A}}^{(1)}(\vec{x})-i\left[W, \boldsymbol{A}_{0}^{(1)}(\vec{x})\right]+m_{q} \boldsymbol{P}^{(1)}(\vec{x})\right] & =-\vec{\nabla} \cdot \overrightarrow{\boldsymbol{A}}^{(0)}(\vec{x})+m_{q} \boldsymbol{P}^{(0)}(\vec{x}) .
\end{aligned}
$$

Notice that the form of the continuity equation we obtain differs from the usual one with $\boldsymbol{V}_{\mu}^{(1)}(\vec{x})=\boldsymbol{A}_{\mu}^{(1)}(\vec{x})=$ $\boldsymbol{P}^{(1)}(\vec{x})=0$. In our case, these terms originate from the additional unitary transformations involving external sources, and they cannot be discarded. ${ }^{4}$ Clearly, $\boldsymbol{V}_{\mu}^{(1)}(\vec{x}), \boldsymbol{A}_{\mu}^{(1)}(\vec{x})$ and $\boldsymbol{P}^{(1)}(\vec{x})$ are proportional to the unitary phases

\footnotetext{
${ }^{4}$ It has already been mentioned in the literature that the continuity equation gets modified if one uses time-dependent unitary transformations [63].
} 
so that the resulting contributions affect only the off-shell behavior of the current operators. In order to avoid the introduction of a set of axial-vector $\left\{\boldsymbol{A}_{\mu}^{(0)}, \boldsymbol{A}_{\mu}^{(1)}\right\}$ and vector $\left\{\boldsymbol{V}_{\mu}^{(0)}, \boldsymbol{V}_{\mu}^{(1)}\right\}$ currents which are not four-vectors individually, we will combine the two current operators into a single one required to be a four-vector, see sections IIF and II G for more details. In momentum space, the current can be defined by

$$
\tilde{V}_{\mu}^{j}\left(\vec{k}, k_{0}\right):=\frac{\delta H_{\mathrm{eff}}}{\delta \tilde{v}_{j}^{\mu}\left(\vec{k}, k_{0}\right)}, \quad \tilde{A}_{\mu}^{j}\left(\vec{k}, k_{0}\right):=\frac{\delta H_{\mathrm{eff}}}{\delta \tilde{a}_{j}^{\mu}\left(\vec{k}, k_{0}\right)}, \quad \tilde{P}{ }^{j}\left(\vec{k}, k_{0}\right):=\frac{\delta H_{\mathrm{eff}}}{\delta \tilde{p} j\left(\vec{k}, k_{0}\right)},
$$

where $H_{\text {eff }}$ is taken at $t=0$ and the functional derivatives are taken at $v=\dot{v}=a=\dot{a}=p=\dot{p}=\dot{s}=s=0, s_{0}=m_{q}$. The Fourier transform for the sources is defined by

$$
v_{\mu}^{j}(x)=: \int d^{4} q \exp (-i q \cdot x) \tilde{v}_{\mu}^{j}(q), \quad a_{\mu}^{j}(x)=: \int d^{4} q \exp (-i q \cdot x) \tilde{a}_{\mu}^{j}(q), \quad p^{j}(x)=: \int d^{4} q \exp (-i q \cdot x) \tilde{p}^{j}(q) .
$$

The currents in Eq. (2.38) can be expressed as a linear combination of the previously defined currents:

$$
\begin{aligned}
\tilde{V}_{\mu}^{j}\left(\vec{k}, k_{0}\right) & =\tilde{V}_{\mu}^{(0) j}(\vec{k})-i k_{0} \tilde{V}_{\mu}^{(1) j}(\vec{k}), \\
\tilde{A}_{\mu}^{j}\left(\vec{k}, k_{0}\right) & =\tilde{A}_{\mu}^{(0) j}(\vec{k})-i k_{0} \tilde{A}_{\mu}^{(1) j}(\vec{k}), \\
\tilde{P}^{j}\left(\vec{k}, k_{0}\right) & =\tilde{P}^{(0) j}(\vec{k})-i k_{0} \tilde{P}^{(1) j}(\vec{k}),
\end{aligned}
$$

where

$$
\begin{aligned}
\tilde{V}_{\mu}^{(l) j}(\vec{k}) & =\int d^{3} x \exp (i \vec{k} \cdot \vec{x}) V_{\mu}^{(l) j}(\vec{x}), \quad \tilde{A}_{\mu}^{(l) j}(\vec{k})=\int d^{3} x \exp (i \vec{k} \cdot \vec{x}) A_{\mu}^{(l) j}(\vec{x}), \\
\tilde{P}^{(l) j}(\vec{k}) & =\int d^{3} x \exp (i \vec{k} \cdot \vec{x}) P^{(l) j}(\vec{x}), \quad l=0,1 .
\end{aligned}
$$

A linear appearance of the energy transfer $k_{0}$ is an off-shell effect and is found to be unavoidable if the currents are to be renormalized. It emerges as a consequence of additional unitary transformations involving external sources, which are needed to maintain renormalizability. The continuity equations given in Eq. (2.37) in coordinate space can be rewritten in momentum space as

$$
\begin{aligned}
{\left[W, \tilde{\boldsymbol{V}}_{0}(\vec{k}, 0)-\frac{\partial}{\partial k_{0}} \vec{k} \cdot \overrightarrow{\tilde{\boldsymbol{V}}}\left(\vec{k}, k_{0}\right)+\frac{\partial}{\partial k_{0}}\left[W, \tilde{\boldsymbol{V}}_{0}\left(\vec{k}, k_{0}\right)\right]\right] } & =\vec{k} \cdot \overrightarrow{\tilde{\boldsymbol{V}}}(\vec{k}, 0) \\
{\left[W, \tilde{\boldsymbol{A}}_{0}(\vec{k}, 0)-\frac{\partial}{\partial k_{0}} \vec{k} \cdot \overrightarrow{\tilde{\boldsymbol{A}}}\left(\vec{k}, k_{0}\right)+\frac{\partial}{\partial k_{0}}\left[W, \tilde{\boldsymbol{A}}{ }_{0}\left(\vec{k}, k_{0}\right)\right]+m_{q} i \frac{\partial}{\partial k_{0}} \tilde{\boldsymbol{P}}\left(\vec{k}, k_{0}\right)\right] } & =\vec{k} \cdot \overrightarrow{\tilde{\boldsymbol{A}}}(\vec{k}, 0)-m_{q} i \tilde{\boldsymbol{P}}(\vec{k}, 0) .
\end{aligned}
$$

The continuity equations are direct consequences of the chiral symmetry and provide non-trivial tests of the derived current operators. Obviously, similar expressions can be found for the singlet vector current related to the $U(1)_{V}$ symmetry (baryon number conservation).

\section{E. Relation to the $S$-matrix}

It is instructive to analyze in detail the relation between the current operators defined in the previous section to $S$-matrix elements. Here and in what follows, we restrict our discussion to the axial currents which are the main focus of our paper.

We begin with the definition of $S$-matrix in the Heisenberg representation,

$$
S=T \exp \left(-i S_{A}[a]\right), \quad \text { with } \quad S_{A}[a]=\int d^{4} x\left[\boldsymbol{A}_{\mu}^{(0) H}(x) \cdot \boldsymbol{a}^{\mu}(x)+\boldsymbol{A}_{\mu}^{(1) H}(x) \cdot \dot{\boldsymbol{a}}^{\mu}(x)\right] .
$$

Here, $T$ denotes the time ordering operator and

$$
\boldsymbol{A}_{\mu}^{(j) H}(x):=\exp \left(i W x_{0}\right) \boldsymbol{A}_{\mu}^{(j)}(\vec{x}) \exp \left(-i W x_{0}\right), \quad j=0,1 .
$$


In linear approximation we can drop the $T$ operator. Sandwiching the $S$-matrix operator between the final and initial states $|\alpha\rangle$ and $|\beta\rangle$, respectively, we get

$$
\langle\alpha|S| \beta\rangle=\langle\alpha \mid \beta\rangle-i\left\langle\alpha\left|S_{A}[a]\right| \beta\right\rangle \text {. }
$$

Using the eigenvalue relations

$$
W|\alpha\rangle=E_{\alpha}|\alpha\rangle \quad \text { and } \quad W|\beta\rangle=E_{\beta}|\beta\rangle
$$

we obtain

$$
\langle\alpha|S| \beta\rangle=\langle\alpha \mid \beta\rangle-i \int d^{4} x \exp \left(i\left(E_{\alpha}-E_{\beta}\right) x_{0}\right)\left(\left\langle\alpha\left|\boldsymbol{A}_{\mu}^{(0)}(\vec{x}) \cdot \boldsymbol{a}^{\mu}\left(x_{0}, \vec{x}\right)\right| \beta\right\rangle+\left\langle\alpha\left|\boldsymbol{A}_{\mu}^{(1)}(\vec{x}) \cdot \dot{\boldsymbol{a}}^{\mu}\left(x_{0}, \vec{x}\right)\right| \beta\right\rangle\right) .
$$

Taking the functional derivative with respect to the sources in momentum space we get

$$
\begin{aligned}
\frac{\delta}{\delta \tilde{a}^{j \mu}\left(k_{0}, \vec{k}\right)}\langle\alpha|S| \beta\rangle & =-i \int d^{4} x \exp \left(i\left(E_{\alpha}-E_{\beta}-k_{0}\right) x_{0}\right) \exp (i \vec{k} \cdot \vec{x})\left(\left\langle\alpha\left|A_{\mu}^{(0) j}(\vec{x})\right| \beta\right\rangle-i k_{0}\left\langle\alpha\left|A_{\mu}^{(1) j}(\vec{x})\right| \beta\right\rangle\right) \\
& =-i 2 \pi \delta\left(E_{\alpha}-E_{\beta}-k_{0}\right)\left(\left\langle\alpha\left|\tilde{A}_{\mu}^{(0) j}(\vec{k})\right| \beta\right\rangle-i k_{0}\left\langle\alpha\left|\tilde{A}_{\mu}^{(1) j}(\vec{k})\right| \beta\right\rangle\right) \\
& =-i 2 \pi \delta\left(E_{\alpha}-E_{\beta}-k_{0}\right)\left\langle\alpha\left|\tilde{A}_{\mu}^{j}\left(k_{0}, \vec{k}\right)\right| \beta\right\rangle .
\end{aligned}
$$

Thus, we see that the current operator, defined in the Schrödinger picture as described above, is indeed identical to the $S$-matrix contribution of the axial-vector current on the energy shell.

Notice that Eq. (2.43) can be rewritten upon performing a partial integration in time

$$
S_{A}[a]=\int d^{4} x\left(\boldsymbol{A}_{\mu}^{(0) H}(x)-\frac{\partial}{\partial t} \boldsymbol{A}_{\mu}^{(1) H}(x)\right) \cdot \boldsymbol{a}^{\mu}(x)=\int d^{4} x\left(\boldsymbol{A}_{\mu}^{(0) H}(x)-i\left[W, \boldsymbol{A}_{\mu}^{(1) H}(x)\right]\right) \cdot \boldsymbol{a}^{\mu}(x) .
$$

This would lead to the following modification of the current

$$
\boldsymbol{A}_{\mu}^{H}(x) \rightarrow \boldsymbol{A}_{\mu}^{\prime H}(x)=\boldsymbol{A}_{\mu}^{(0) H}(x)-i\left[W, \boldsymbol{A}_{\mu}^{(1) H}(x)\right] .
$$

Notice that the resulting current operator $\boldsymbol{A}_{\mu}^{\prime}(\vec{x})=\boldsymbol{A}_{\mu}^{\prime H}\left(\vec{x}, x_{0}=0\right)$ satisfies the usual, non-modified continuity equation

$$
i\left[W, \boldsymbol{A}_{0}^{\prime}(\vec{x})\right]=-\vec{\nabla} \cdot \vec{A}^{\prime}(\vec{x})+2 m_{q} \boldsymbol{P}^{\prime}(\vec{x})
$$

where, similar to the axial-vector current, we have introduced the corresponding modified version of the pseudoscalar current

$$
\boldsymbol{P}^{\prime}(\vec{x})=\boldsymbol{P}^{(0)}(\vec{x})-i\left[W, \boldsymbol{P}^{(1)}(\vec{x})\right]
$$

Eq. (2.51) is obtained straightforwardly by inserting the definitions of Eqs. (2.50) and (2.52) in Eq. (2.37). The current $\boldsymbol{A}_{\mu}^{\prime H}$ is, however, identical to the current without additional unitary transformations involving external fields. To see this we consider the axial-vector current contribution to the effective Hamiltonian before applying the unitary transformation involving external fields

$$
U_{\eta}^{\dagger} U_{\text {Okubo }}^{\dagger} H[a] U_{\text {Okubo }} U_{\eta}=W+\int d^{3} x \boldsymbol{B}_{\mu}(\vec{x}) \cdot \boldsymbol{a}^{\mu}\left(x_{0}, \vec{x}\right)
$$

Parametrizing an additional unitary transformation with external sources by

$$
U[a]=1-\int d^{3} x \boldsymbol{C}_{\mu}(\vec{x}) \cdot \boldsymbol{a}^{\mu}\left(x_{0}, \vec{x}\right)+\mathcal{O}\left(a^{2}\right),
$$

where $\boldsymbol{C}_{\mu}$ is an antihermitian field operator, we get

$$
\eta U^{\dagger}[a] U_{\eta}^{\dagger} U_{\text {Okubo }}^{\dagger} H[a] U_{\text {Okubo }} U_{\eta} U[a] \eta+i \eta\left(\frac{\partial}{\partial x_{0}} U^{\dagger}[a]\right) U[a] \eta=W+\int d^{3} x\left[\left(\boldsymbol{B}_{\mu}(\vec{x})+\left[\boldsymbol{C}_{\mu}(\vec{x}), W\right]\right) \cdot \boldsymbol{a}^{\mu}\left(x_{0}, \vec{x}\right)\right.
$$




$$
\left.+i \boldsymbol{C}_{\mu}(\vec{x}) \cdot \dot{\boldsymbol{a}}^{\mu}\left(x_{0}, \vec{x}\right)\right]
$$

We then read off

$$
\boldsymbol{A}_{\mu}^{(0)}(\vec{x})=\boldsymbol{B}_{\mu}(\vec{x})+\left[\boldsymbol{C}_{\mu}(\vec{x}), W\right] \quad \text { and } \quad \boldsymbol{A}_{\mu}^{(1)}(\vec{x})=i \boldsymbol{C}_{\mu}(\vec{x})
$$

and conclude

$$
\boldsymbol{A}_{\mu}^{\prime H}(x)=\boldsymbol{B}_{\mu}^{H}(x)+\left[\boldsymbol{C}_{\mu}^{H}(x), W\right]+\left[W, \boldsymbol{C}_{\mu}^{H}(x)\right]=\boldsymbol{B}_{\mu}^{H}(x)
$$

As already pointed out above, additional unitary transformations involving external sources are needed for the renormalization of the current. Since their effects are switched off in the current $\boldsymbol{A}_{\mu}^{\prime}$, we prefer to work with the current $\boldsymbol{A}_{\mu}$ in order to have properly renormalized current operators.

\section{F. Poincaré invariance constraints}

It is instructive to analyze the four-vector constraint on $\boldsymbol{A}_{\mu}$. In the Heisenberg picture, this means

$$
\exp (-i \vec{e} \cdot \vec{K} \theta) \boldsymbol{A}_{\mu}^{H}(x) \exp (i \vec{e} \cdot \vec{K} \theta)=\Lambda_{\mu}{ }^{\nu}(\theta) \boldsymbol{A}_{\nu}^{H}\left(\Lambda^{-1}(\theta) x\right)
$$

where $\vec{K}$ is a boost operator, $\vec{e}$ is a boost direction and $\Lambda$ is a $4 \times 4$ boost matrix which depends on the boost direction $\vec{e}$ and a boost angle $\theta$ :

$$
\Lambda(\theta)\left(\begin{array}{c}
x_{0} \\
\vec{x}
\end{array}\right)=\left(\begin{array}{c}
x_{0} \cosh (\theta)+\vec{e} \cdot \vec{x} \sinh (\theta) \\
\vec{x}+\vec{e} x_{0} \sinh (\theta)+\vec{e}(\cosh (\theta)-1) \vec{e} \cdot \vec{x}
\end{array}\right)=\left(\begin{array}{c}
x_{0} \\
\vec{x}
\end{array}\right)+\theta\left(\begin{array}{c}
\vec{e} \cdot \vec{x} \\
\vec{e} x_{0}
\end{array}\right)+\mathcal{O}\left(\theta^{2}\right) .
$$

For a given four-vector $x=\left(x^{0}, \vec{x}\right)$, we can introduce an orthogonal four-vector via

$$
x^{\perp}=\left(\vec{e} \cdot \vec{x}, \vec{e} x_{0}\right),
$$

so that a Lorentz transformation has the form

$$
\Lambda(\theta) x=x+\theta x^{\perp}+\mathcal{O}\left(\theta^{2}\right) .
$$

Since in coordinate space the current $\boldsymbol{A}_{\mu}^{H}(x)$ is given in terms of $\boldsymbol{A}_{\mu}^{(0)}$ and $\boldsymbol{A}_{\mu}^{(1)}$, we rewrite this relation as

$$
\exp (-i \vec{e} \cdot \vec{K} \theta) S_{A}[a] \exp (i \vec{e} \cdot \vec{K} \theta)=S_{A}\left[a^{\prime}\right], \quad \boldsymbol{a}^{\prime \mu}(x)=\left(\Lambda^{-1}\right)^{\mu}{ }_{\nu}(\theta) \boldsymbol{a}^{\nu}(\Lambda(\theta) x),
$$

where $S_{A}[a]$ is defined in Eq. (2.43). The time derivative of $\boldsymbol{a}^{\prime}$ is given by

$$
\dot{\boldsymbol{a}}^{\prime \mu}(x)=\left.\left(\Lambda^{-1}\right)_{\nu}^{\mu}(\theta) \frac{\partial}{\partial y^{\alpha}} \boldsymbol{a}^{\nu}(y)\right|_{y=\Lambda(\theta) x} \Lambda_{0}^{\alpha}(\theta) .
$$

We now make the substitution $x \rightarrow \Lambda^{-1}(\theta) x$ in the integral appearing in the definition of $S_{A}\left[a^{\prime}\right]$ to obtain

$$
\begin{aligned}
& \exp (-i \vec{e} \cdot \vec{K} \theta) S_{A}[a] \exp (i \vec{e} \cdot \vec{K} \theta) \\
& =\Lambda_{\mu}{ }^{\nu}(\theta) \int d^{4} x\left(\boldsymbol{A}_{\nu}^{(0) H}\left(\Lambda^{-1}(\theta) x\right) \cdot \boldsymbol{a}^{\mu}(x)+\boldsymbol{A}_{\nu}^{(1) H}\left(\Lambda^{-1}(\theta) x\right) \cdot \frac{\partial}{\partial x^{\alpha}} \boldsymbol{a}^{\mu}(x) \Lambda_{0}^{\alpha}(\theta)\right) .
\end{aligned}
$$

For an infinitesimally small $\theta$, this leads to

$$
\begin{aligned}
\frac{\partial}{\partial x^{\alpha}} \boldsymbol{a}^{\mu}(x) \Lambda_{0}^{\alpha}(\theta) & =\left(\Lambda^{-1}\right)_{0}{ }^{\alpha}(\theta) \frac{\partial}{\partial x^{\alpha}} \boldsymbol{a}^{\mu}(x)=\dot{\boldsymbol{a}}^{\mu}(x)+\theta \vec{e} \cdot \vec{\nabla} \boldsymbol{a}^{\mu}(x), \\
\boldsymbol{A}_{\nu}^{(l) H}\left(\Lambda^{-1}(\theta) x\right) & =\boldsymbol{A}_{\nu}^{(l) H}(x)-\theta x_{\alpha}^{\perp} \frac{\partial}{\partial x_{\alpha}} \boldsymbol{A}_{\nu}^{(l) H}(x) .
\end{aligned}
$$


Applying this to Eq. (2.64) we get

$$
\begin{aligned}
-i\left[\vec{e} \cdot \vec{K}, S_{A}[a]\right] & =S_{A^{\perp}}[a]-\int d^{4} x\left[x_{\nu}^{\perp}\left(\frac{\partial}{\partial x_{\nu}} \boldsymbol{A}_{\mu}^{(0) H}(x)\right) \cdot \boldsymbol{a}^{\mu}(x)+x_{\nu}^{\perp}\left(\frac{\partial}{\partial x_{\nu}} \boldsymbol{A}_{\mu}^{(1) H}(x)\right) \cdot \dot{\boldsymbol{a}}^{\mu}(x)\right. \\
& \left.-\boldsymbol{A}_{\mu}^{(1) H}(x) \cdot\left(\vec{e} \cdot \vec{\nabla} \boldsymbol{a}^{\mu}(x)\right)\right],
\end{aligned}
$$

with

$$
S_{A^{\perp}}[a]=\int d^{4} x\left(\boldsymbol{A}_{\mu}^{(0) H \perp}(x) \cdot \boldsymbol{a}^{\mu}(x)+\boldsymbol{A}_{\mu}^{(1) H \perp}(x) \cdot \dot{\boldsymbol{a}}^{\mu}(x)\right) .
$$

After a partial integration in the spatial components, one obtains

$$
\begin{aligned}
-i\left[\vec{e} \cdot \vec{K}, S_{A}[a]\right] & =\int d^{4} x\left[\left(\boldsymbol{A}_{\mu}^{(0) H \perp}(x)-x_{\nu}^{\perp} \frac{\partial}{\partial x_{\nu}} \boldsymbol{A}_{\mu}^{(0) H}(x)-\vec{e} \cdot \vec{\nabla} \boldsymbol{A}_{\mu}^{(1) H}(x)\right) \cdot \boldsymbol{a}^{\mu}(x)\right. \\
& \left.\left.+\int \boldsymbol{A}_{\mu}^{(1) H \perp}(x)-x_{\nu}^{\perp} \frac{\partial}{\partial x_{\nu}} \boldsymbol{A}_{\mu}^{(1) H}(x)\right) \cdot \dot{\boldsymbol{a}}^{\mu}(x)\right] \\
& =\int d^{4} x\left[\left(\boldsymbol{A}_{\mu}^{(0) H \perp}(x)-i \vec{e} \cdot \vec{x}\left[W, \boldsymbol{A}_{\mu}^{(0) H}(x)\right]-x_{0} \vec{e} \cdot \vec{\nabla} \boldsymbol{A}_{\mu}^{(0) H}(x)-\vec{e} \cdot \vec{\nabla} \boldsymbol{A}_{\mu}^{(1) H}(x)\right) \cdot \boldsymbol{a}^{\mu}(x)\right. \\
& \left.+\left(\boldsymbol{A}_{\mu}^{(1) H \perp}(x)-i \vec{e} \cdot \vec{x}\left[W, \boldsymbol{A}_{\mu}^{(1) H}(x)\right]-x_{0} \vec{e} \cdot \vec{\nabla} \boldsymbol{A}_{\mu}^{(1) H}(x)\right) \cdot \dot{\boldsymbol{a}}^{\mu}(x)\right] .
\end{aligned}
$$

To proceed further, we need the following commutation relations

$$
\left[W, K_{i}\right]=-i P_{i} \quad \text { and } \quad\left[W, P_{i}\right]=0
$$

from the Poincaré algebra. Using Hadamard's lemma

$$
\exp (A) B \exp (-A)=B+[A, B]+\frac{1}{2}[A,[A, B]]+\frac{1}{3 !}[A,[A,[A, B]]]+\ldots,
$$

which is valid for any square matrices $A$ and $B$, we get

$$
\exp \left(-i W x_{0}\right) \vec{e} \cdot \vec{K} \exp \left(i W x_{0}\right)=\vec{e} \cdot \vec{K}-i x_{0}[W, \vec{e} \cdot \vec{K}]=\vec{e} \cdot \vec{K}-x_{0} \vec{e} \cdot \vec{P} .
$$

Notice that all higher commutators vanish due to $\left[W, P_{i}\right]=0$. We conclude that

$$
\begin{aligned}
-i\left[\vec{e} \cdot \vec{K}, \boldsymbol{A}_{\mu}^{(l) H}(x)\right] & =-i \exp \left(i W x_{0}\right)\left[\exp \left(-i W x_{0}\right) \vec{e} \cdot \vec{K} \exp \left(i W x_{0}\right), \boldsymbol{A}_{\mu}^{(l)}(\vec{x})\right] \exp \left(-i W x_{0}\right) \\
& =-i \exp \left(i W x_{0}\right)\left[\vec{e} \cdot \vec{K}-x_{0} \vec{e} \cdot \vec{P}, \boldsymbol{A}_{\mu}^{(l)}(\vec{x})\right] \exp \left(-i W x_{0}\right) .
\end{aligned}
$$

Using the relation

$$
\left[i \vec{e} \cdot \vec{P}, \boldsymbol{A}_{\mu}^{(l)}(\vec{x})\right]=-\vec{e} \cdot \vec{\nabla} \boldsymbol{A}_{\mu}^{(l)}(\vec{x}),
$$

which follows from a general translation

$$
\boldsymbol{A}_{\mu}^{(l)}(\vec{x})=\exp (-i \vec{P} \cdot \vec{x}) \boldsymbol{A}_{\mu}^{(l)}(0) \exp (i \vec{P} \cdot \vec{x}),
$$

we can rewrite Eq. (2.72) as

$$
-i\left[\vec{e} \cdot \vec{K}, \boldsymbol{A}_{\mu}^{(l) H}(x)\right]=-i \exp \left(i W x_{0}\right)\left[\vec{e} \cdot \vec{K}, \boldsymbol{A}_{\mu}^{(l)}(\vec{x})\right] \exp \left(-i W x_{0}\right)-x_{0} \vec{e} \cdot \vec{\nabla} \boldsymbol{A}_{\mu}^{(l) H}(x) .
$$

With this relation, we can finally bring Eq. (2.68) into the form

$$
-i \int d^{4} x \exp \left(i W x_{0}\right)\left(\left[\vec{e} \cdot \vec{K}, \boldsymbol{A}_{\mu}^{(0)}(\vec{x})\right] \cdot \boldsymbol{a}^{\mu}(x)+\left[\vec{e} \cdot \vec{K}, \boldsymbol{A}_{\mu}^{(1)}(\vec{x}) \cdot \dot{\boldsymbol{a}}^{\mu}(x)\right]\right) \exp \left(-i W x_{0}\right)
$$




$$
\begin{aligned}
= & \int d^{4} x\left[\left(\boldsymbol{A}_{\mu}^{(0) H \perp}(x)-i \vec{e} \cdot \vec{x}\left[W, \boldsymbol{A}_{\mu}^{(0) H}(x)\right]-\vec{e} \cdot \vec{\nabla} \boldsymbol{A}_{\mu}^{(1) H}(x)\right) \cdot \boldsymbol{a}^{\mu}(x)\right. \\
& \left.+\left(\boldsymbol{A}_{\mu}^{(1) H \perp}(x)-i \vec{e} \cdot \vec{x}\left[W, \boldsymbol{A}_{\mu}^{(1) H}(x)\right]\right) \cdot \dot{\boldsymbol{a}}^{\mu}(x)\right] .
\end{aligned}
$$

Sandwiching this relation between the final and initial states $|\alpha\rangle$ and $|\beta\rangle$, respectively, and taking the functional derivative with respect to the axial-vector source in momentum space on both sides, we end up with our final result

$$
\begin{aligned}
& 2 \pi \delta\left(E_{\alpha}-E_{\beta}-k_{0}\right)\left\langle\alpha\left|\left[-i \vec{e} \cdot \vec{K}, \tilde{\boldsymbol{A}}_{\mu}^{(0)}(\vec{k})-i k_{0} \tilde{\boldsymbol{A}}_{\mu}^{(1)}(\vec{k})\right]\right| \beta\right\rangle \\
= & \left.2 \pi \delta\left(E_{\alpha}-E_{\beta}-k_{0}\right)\langle\alpha|\left(\tilde{\boldsymbol{A}}_{\mu}^{(0) \perp}(\vec{k})-i k_{0} \tilde{\boldsymbol{A}}_{\mu}^{(1) \perp}(\vec{k})-\vec{e} \cdot \vec{\nabla}_{k}\left[W, \tilde{\boldsymbol{A}}_{\mu}^{(0)}(\vec{k})-i k_{0} \tilde{\boldsymbol{A}}_{\mu}^{(1)}(\vec{k})\right]\right)+i \vec{e} \cdot \vec{k} \tilde{\boldsymbol{A}}_{\mu}^{(1)}(\vec{k})\right)|\beta\rangle .
\end{aligned}
$$

We can also write this relation in the operator form as

$$
-i\left[\vec{e} \cdot \vec{K}, \tilde{\boldsymbol{A}}_{\mu}(k)\right]=\tilde{\boldsymbol{A}}_{\mu}^{\perp}(k)-\vec{e} \cdot \vec{\nabla}_{k}\left[W, \tilde{\boldsymbol{A}}_{\mu}(k)\right]-\vec{e} \cdot \vec{k} \frac{\partial}{\partial k_{0}} \tilde{\boldsymbol{A}}_{\mu}(k)+i\left[W, \boldsymbol{X}_{\mu}\right]-i k_{0} \boldsymbol{X}_{\mu}
$$

where $X_{\mu}$ is an arbitrary operator satisfying

$$
\lim _{k_{0} \rightarrow E_{\beta}-E_{\alpha}}\left(k_{0}+E_{\alpha}-E_{\beta}\right)\left\langle\beta\left|\boldsymbol{X}_{\mu}\right| \alpha\right\rangle=0 .
$$

\section{G. Effective boost operator}

In order to explicitly verify the four-vector condition of Eq. (2.77) we need to construct the boost operator $\vec{K}$. As usual, we start from the classical conserved Noether current which is a reflection of the fact that proper orthochronous Lorentz transformations represent the symmetry of any relativistic field theory. An infinitesimal proper orthochronous Lorentz transformation, which is a combination of a rotation and a boost, is given by

$$
x_{\mu} \rightarrow x_{\mu}+\epsilon_{\mu \nu} x^{\nu}
$$

with $\epsilon_{\mu \nu}=-\epsilon_{\nu \mu}$ an antisymmetric infinitesimal angle. Consider a field transformation

$$
\begin{aligned}
\pi^{a}(x) & \rightarrow \pi^{\prime a}(x)=\pi^{a}\left(\Lambda^{-1} x\right)=\pi^{a}\left(x-\epsilon \cdot x+\mathcal{O}\left(\epsilon^{2}\right)\right) \\
\partial_{\mu} \pi^{a}(x) & \rightarrow \partial_{\mu} \pi^{\prime a}(x)=\left(\frac{\partial}{\partial y^{\mu}} \pi^{a}(y)-\epsilon_{\alpha \mu} \frac{\partial}{\partial y_{\alpha}} \pi^{a}(y)+\mathcal{O}\left(\epsilon^{2}\right)\right)_{y=x-\epsilon \cdot x+\mathcal{O}\left(\epsilon^{2}\right)} \\
N_{i}(x) & \rightarrow N_{i}^{\prime}(x)=\left(1+\frac{1}{2} \epsilon_{\mu \nu} \Sigma^{\mu \nu}+\mathcal{O}\left(\epsilon^{2}\right)\right) N_{i}\left(x-\epsilon \cdot x+\mathcal{O}\left(\epsilon^{2}\right)\right) \\
\partial_{\mu} N_{i}(x) & \left.\rightarrow \partial_{\mu} N_{i}^{\prime}(x)=\left(\frac{\partial}{\partial y^{\mu}} N_{i}(y)+\frac{1}{2} \epsilon_{\alpha \beta} \Sigma^{\alpha \beta} \frac{\partial}{\partial y^{\mu}} N_{i}(y)-\epsilon_{\alpha \mu} \frac{\partial}{\partial y_{\alpha}} N_{i}(y)+\mathcal{O}\left(\epsilon^{2}\right)\right)\right)_{\left.y=x-\epsilon \cdot x+\mathcal{O}\left(\epsilon^{2}\right)\right)},
\end{aligned}
$$

where $i$ is the isospin index of the nucleon,

$$
\Sigma^{\mu \nu}=\frac{i}{2}\left[\gamma^{\mu}, \gamma^{\nu}\right]
$$

and the $\gamma^{\mu}$ are the Dirac matrices. If we set all external sources to zero (or to $m_{q}$ in the case of the scalar source), the effective chiral Lagrangian density does not depend explicitly on $x$, and we get

$$
\left.\frac{\partial}{\partial \epsilon^{\nu \sigma}} \mathcal{L}_{\mathrm{ChPT}}\left(\boldsymbol{\pi}^{\prime}, N^{\prime}\right)\right|_{\epsilon=0}=-\left(x_{\sigma} \partial_{\nu}-x_{\nu} \partial_{\sigma}\right) \mathcal{L}_{\mathrm{ChPT}}(\boldsymbol{\pi}, N)=\partial_{\mu}\left(x_{\nu} g^{\mu}{ }_{\sigma}-x_{\sigma} g^{\mu}{ }_{\nu}\right) \mathcal{L}_{\mathrm{ChPT}}(\boldsymbol{\pi}, N)=: \partial_{\mu} F_{\nu \sigma}^{\mu},
$$

where we assume that the chiral Lagrangian density is a Lorentz scalar:

$$
\partial_{\mu} \pi^{a}(x) \rightarrow \partial_{\mu} \pi^{a}(x)-\epsilon_{\alpha \mu} \partial^{\alpha} \pi^{a}(x)+\mathcal{O}\left(\epsilon^{2}\right),
$$




$$
\begin{aligned}
N_{i}(x) & \rightarrow N_{i}(x)+\frac{1}{2} \epsilon_{\mu \nu} \Sigma^{\mu \nu} N_{i}(x)+\mathcal{O}\left(\epsilon^{2}\right), \\
\partial_{\mu} N_{i}(x) & \rightarrow \partial_{\mu} N_{i}(x)+\frac{1}{2} \epsilon_{\alpha \beta} \Sigma^{\alpha \beta} \partial_{\mu} N_{i}(x)-\epsilon_{\alpha \mu} \partial^{\alpha} N_{i}(x)+\mathcal{O}\left(\epsilon^{2}\right) .
\end{aligned}
$$

The conserved classical Noether current is given by

$$
\mathcal{M}_{c}^{\mu \alpha \beta}=\frac{\partial \mathcal{L}_{\mathrm{ChPT}}}{\partial \partial_{\mu} \pi^{a}(x)} D^{\alpha \beta} \pi^{a}(x)+\frac{\partial \mathcal{L}_{\mathrm{ChPT}}}{\partial \partial_{\mu} N_{i}(x)} D^{\alpha \beta} N_{i}(x)+D^{\beta \alpha} \bar{N}_{i}(x) \frac{\partial \mathcal{L}_{\mathrm{ChPT}}}{\partial \partial_{\mu} \bar{N}_{i}(x)}-F^{\mu \alpha \beta}(x),
$$

where the subscript " $c$ " stays for canonical and

$$
\begin{aligned}
& D^{\alpha \beta} \pi^{a}(x)=\left.\frac{\partial}{\partial \epsilon_{\alpha \beta}} \pi^{\prime a}(x)\right|_{\epsilon=0}=x^{\alpha} \partial^{\beta} \pi^{a}(x)-x^{\beta} \partial^{\alpha} \pi^{a}(x), \\
& D^{\alpha \beta} N_{i}(x)=\left.\frac{\partial}{\partial \epsilon_{\alpha \beta}} N_{i}^{\prime a}(x)\right|_{\epsilon=0}=\left(\Sigma^{\alpha \beta}+x^{\alpha} \partial^{\beta}-x^{\beta} \partial^{\alpha}\right) N_{i}(x) .
\end{aligned}
$$

In terms of the energy-momentum tensor

$$
T^{\mu \nu}=\frac{\partial \mathcal{L}_{\mathrm{ChPT}}}{\partial \partial_{\mu} \pi^{a}(x)} \partial^{\nu} \pi^{a}(x)+\frac{\partial \mathcal{L}_{\mathrm{ChPT}}}{\partial \partial_{\mu} N_{i}(x)} \partial^{\nu} N_{i}(x)+\partial^{\nu} \bar{N}_{i}(x) \frac{\partial \mathcal{L}_{\mathrm{ChPT}}}{\partial \partial_{\mu} \bar{N}_{i}(x)}-g^{\mu \nu} \mathcal{L}_{\mathrm{ChPT}},
$$

$\mathcal{M}_{c}^{\mu \alpha \beta}$ is given by

$$
\mathcal{M}_{c}^{\mu \alpha \beta}=x^{\alpha} T^{\mu \beta}-x^{\beta} T^{\mu \alpha}+\frac{\partial \mathcal{L}_{\mathrm{ChPT}}}{\partial \partial_{\mu} N_{i}(x)} \Sigma^{\alpha \beta} N_{i}(x)+\bar{N}_{i}(x) \Sigma^{\beta \alpha} \frac{\partial \mathcal{L}_{\mathrm{ChPT}}}{\partial \partial_{\mu} \bar{N}_{i}(x)} .
$$

Due to current conservation

$$
\partial_{\mu} \mathcal{M}_{c}^{\mu \alpha \beta}=0
$$

there are six conserved charges: the boost

$$
K_{c}^{j}=\int d^{3} x \mathcal{M}_{c}^{0 j 0}=\int d^{3} x\left(x^{j} T^{00}-x^{0} T^{0 j}+\frac{\partial \mathcal{L}_{\mathrm{ChPT}}}{\partial \partial_{0} N_{i}(x)} \Sigma^{j 0} N_{i}(x)+\bar{N}_{i}(x) \Sigma^{0 j} \frac{\partial \mathcal{L}_{\mathrm{ChPT}}}{\partial \partial_{0} \bar{N}_{i}(x)}\right)
$$

and the angular momentum

$$
J_{c}^{k}=\frac{1}{2} \epsilon^{i j k} \int d^{3} x \mathcal{M}_{c}^{0 i j}=\frac{1}{2} \epsilon^{i j k} \int d^{3} x\left(x^{i} T_{c}^{0 j}-x^{j} T_{c}^{0 i}+\frac{\partial \mathcal{L}_{\mathrm{ChPT}}}{\partial \partial_{0} N_{n}(x)} \Sigma^{i j} N_{n}(x)+\bar{N}_{n}(x) \Sigma^{j i} \frac{\partial \mathcal{L}_{\mathrm{ChPT}}}{\partial \partial_{0} \bar{N}_{n}(x)}\right) .
$$

In this notation, the boost appears to explicitly depend on the spin $\Sigma^{0 j}$. One can, however, give a simpler form of the boost if one uses the Belinfante energy-momentum tensor

$$
\Theta^{\mu \nu}(x)=T^{\mu \nu}(x)+\frac{1}{2} \partial_{\alpha} X^{\alpha \mu \nu}(x),
$$

which is assumed to be symmetric under the interchange $\mu \leftrightarrow \nu$. The tensor $X^{\alpha \mu \nu}$ has to be antisymmetric under the $\alpha \leftrightarrow \mu$ interchange,

$$
X^{\alpha \mu \nu}(x)=-X^{\mu \alpha \nu}(x),
$$

in order to maintain the current conservation relation

$$
\partial_{\mu} \Theta^{\mu \nu}=\partial_{\mu} T^{\mu \nu}=0
$$

The symmetry requirement under the interchange $\mu \leftrightarrow \nu$ gives a constraint on the tensor $X^{\lambda \mu \nu}$

$$
0=\Theta^{\mu \nu}(x)-\Theta^{\nu \mu}(x)=T^{\mu \nu}(x)-T^{\nu \mu}(x)+\frac{1}{2} \partial_{\alpha}\left(X^{\alpha \mu \nu}(x)-X^{\alpha \nu \mu}(x)\right) .
$$

Due to the invariance of the Lagrangian under the transformation in Eq. (2.84), we get

$$
T^{\mu \nu}-T^{\nu \mu}=-\frac{\partial \mathcal{L}_{\mathrm{ChPT}}}{\partial \partial_{\alpha} N_{i}(x)} \Sigma^{\mu \nu} \partial_{\alpha} N_{i}(x)-\bar{N}_{i}(x) \overleftarrow{\partial}_{\alpha} \Sigma^{\nu \mu} \frac{\partial \mathcal{L}_{\mathrm{ChPT}}}{\partial \partial_{\alpha} \bar{N}_{i}(x)}-\frac{\partial \mathcal{L}_{\mathrm{ChPT}}}{\partial N_{i}(x)} \Sigma^{\mu \nu} N_{i}(x)-\bar{N}_{i}(x) \Sigma^{\nu \mu} \frac{\partial \mathcal{L}_{\mathrm{ChPT}}}{\partial \bar{N}_{i}(x)}
$$




$$
=-\partial_{\alpha}\left(\frac{\partial \mathcal{L}_{\mathrm{ChPT}}}{\partial \partial_{\alpha} N_{i}(x)} \Sigma^{\mu \nu} N_{i}(x)+\bar{N}_{i}(x) \Sigma^{\nu \mu} \frac{\partial \mathcal{L}_{\mathrm{ChPT}}}{\partial \partial_{\alpha} \bar{N}_{i}(x)}\right)
$$

where we have used the equation of motion in the second line. Matching Eqs. (2.95) and (2.96), we obtain the relation

$$
X^{\alpha \mu \nu}-X^{\alpha \nu \mu}=2\left(\frac{\partial \mathcal{L}_{\mathrm{ChPT}}}{\partial \partial_{\alpha} N_{i}(x)} \Sigma^{\mu \nu} N_{i}(x)+\bar{N}_{i}(x) \Sigma^{\nu \mu} \frac{\partial \mathcal{L}_{\mathrm{ChPT}}}{\partial \partial_{\alpha} \bar{N}_{i}(x)}\right)
$$

The ansatz

$$
\begin{aligned}
X^{\alpha \mu \nu} & =\frac{\partial \mathcal{L}_{\mathrm{ChPT}}}{\partial \partial_{\alpha} N_{i}(x)} \Sigma^{\mu \nu} N_{i}(x)-\frac{\partial \mathcal{L}_{\mathrm{ChPT}}}{\partial \partial_{\mu} N_{i}(x)} \Sigma^{\alpha \nu} N_{i}(x)+\frac{\partial \mathcal{L}_{\mathrm{ChPT}}}{\partial \partial_{\nu} N_{i}(x)} \Sigma^{\mu \alpha} N_{i}(x) \\
& +\bar{N}_{i}(x) \Sigma^{\nu \mu} \frac{\partial \mathcal{L}_{\mathrm{ChPT}}}{\partial \partial_{\alpha} \bar{N}_{i}(x)}-\bar{N}_{i}(x) \Sigma^{\nu \alpha} \frac{\partial \mathcal{L}_{\mathrm{ChPT}}}{\partial \partial_{\mu} \bar{N}_{i}(x)}+\bar{N}_{i}(x) \Sigma^{\alpha \mu} \frac{\partial \mathcal{L}_{\mathrm{ChPT}}}{\partial \partial_{\nu} \bar{N}_{i}(x)}
\end{aligned}
$$

solves Eq. (2.97). Given that $X^{\alpha \mu \nu}=X^{\alpha \nu \mu}$ by construction, only the antisymmetric part survives on the right-hand side of Eq. (2.97). In terms of $X^{\alpha \mu \nu}$, we can rewrite the angular momentum tensor as

$$
\mathcal{M}_{c}^{\mu \alpha \beta}=x^{\alpha} T^{\mu \beta}-x^{\beta} T^{\mu \alpha}+\frac{1}{2}\left(X^{\mu \alpha \beta}-X^{\mu \beta \alpha}\right) .
$$

One can now redefine the angular momentum tensor as

$$
\begin{aligned}
\mathcal{M}^{\mu \alpha \beta} & =\mathcal{M}_{c}^{\mu \alpha \beta}+\frac{1}{2} \partial_{\lambda}\left(x^{\alpha} X^{\lambda \mu \beta}-x^{\beta} X^{\lambda \mu \alpha}\right) \\
& =\mathcal{M}_{c}^{\mu \alpha \beta}+\frac{1}{2}\left(x^{\alpha} \partial_{\lambda} X^{\lambda \mu \beta}-x^{\beta} \partial_{\lambda} X^{\lambda \mu \alpha}\right)+\frac{1}{2}\left(X^{\alpha \mu \beta}-X^{\beta \mu \alpha}\right),
\end{aligned}
$$

which leads to

$$
\mathcal{M}^{\mu \alpha \beta}=x^{\alpha} \Theta^{\mu \beta}-x^{\beta} \Theta^{\mu \alpha}
$$

The current $\mathcal{M}^{\mu \alpha \beta}$ is conserved since

$$
\partial_{\mu} \mathcal{M}^{\mu \alpha \beta}=\frac{1}{2} \partial_{\mu} \partial_{\lambda}\left(x^{\alpha} X^{\lambda \mu \beta}-x^{\beta} X^{\lambda \mu \alpha}\right)=0 .
$$

The last equation is valid since the tensor in the bracket is antisymmetric under the interchange $\lambda \leftrightarrow \mu$. We obtain the time-independent boost function

$$
K^{j}=\int d^{3} x\left(x^{j} \Theta^{00}-x^{0} \Theta^{0 j}\right)
$$

from the conserved angular momentum tensor $\mathcal{M}^{\mu \alpha \beta}$, while the angular momentum function has the form

$$
J^{k}=\frac{1}{2} \epsilon^{i j k} \int d^{3} x \mathcal{M}^{0 i j}=\frac{1}{2} \epsilon^{i j k} \int d^{3} x\left(x^{i} \Theta^{0 j}-x^{j} \Theta^{0 i}\right) .
$$

Notice that due to antisymmetry of $X^{\lambda \mu \beta}$ under the interchange $\lambda \leftrightarrow \mu$, one gets

$$
\partial_{0}\left(x^{\alpha} X^{00 \beta}-x^{\beta} X^{00 \alpha}\right)=0 .
$$

For this reason,

$$
K^{j}=K_{c}^{j} \quad \text { and } \quad J^{k}=J_{c}^{k} .
$$

We see that with the symmetric energy-momentum tensor, the definition of the boost function becomes more compact. This, however, is just a matter of notation. In either case, at $x_{0}=0$ the boost operator has a contribution from the Hamiltonian density $T^{00}$ and and from spin part proportional to $\Sigma^{0 j}$.

Once we defined the boost function, we take the same definition in quantum field theory by promoting fields to operators. In the final step, we need to block diagonalize the boost operator $K^{j}$ in Fock space. As has been shown in Ref. [64, 65] the Hamiltonian and boost operators get block diagonalized simultaneously by the Okubo unitary 
transformation. The proof in Ref. [64] was, however, restricted to Yukawa-like interactions that are linear in meson fields. This is certainly not enough for chiral EFT. Fortunately, the algebraic considerations of that work, which are based on commutation relations of the Poincaré algebra, can be generalized to any interaction. We give the somewhat lengthy proof of this statement in Appendix A. In order to calculate an effective boost operator acting on the nucleonic part of the Fock space, we follow our usual strategy by first applying the Okubo transformation on the chiral boost operator and subsequently performing additional unitary transformations on the $\eta$ space, which do not depend on the external sources. The resulting effective boost operator is given by

$$
K_{\mathrm{eff}}^{j}=\eta U_{\eta}^{\dagger} U_{\text {Okubo }}^{\dagger} K^{j} U_{\text {Okubo }} U_{\eta} \eta
$$

Due to translational invariance of the unitary transformations $U_{\eta}$ and $U_{\text {Okubo, we can write the boost as }}$

$$
\left\langle\alpha\left|K^{j}\right| \beta\right\rangle=(2 \pi)^{3}\left(i \frac{\partial}{\partial P_{\alpha}^{j}} \delta\left(\vec{P}_{\alpha}-\vec{P}_{\beta}\right)\right)\left\langle\alpha\left|\eta U_{\eta}^{\dagger} U_{\text {Okubo }}^{\dagger} \Theta^{00}(0) U_{\text {Okubo }} U_{\eta} \eta\right| \beta\right\rangle .
$$

Here $|\alpha\rangle$ and $|\beta\rangle$ are chosen to be eigenstates of the free Hamiltonian $H_{0}$, and the total momentum is an eigenvalue of the momentum operator

$$
P^{j}|\alpha\rangle=P_{\alpha}^{j}|\alpha\rangle, \quad P^{j}|\beta\rangle=P_{\beta}^{j}|\beta\rangle, \quad H_{0}|\alpha\rangle=E_{\alpha}|\alpha\rangle, \quad H_{0}|\beta\rangle=E_{\beta}|\beta\rangle .
$$

In this notation, the boost and Hamiltonian matrix elements look very similar. Indeed, the effective Hamiltonian is given by

$$
\left\langle\alpha\left|H_{0}+V\right| \beta\right\rangle=(2 \pi)^{3} \delta\left(\vec{P}_{\alpha}-\vec{P}_{\beta}\right)\left\langle\alpha\left|\eta U_{\eta}^{\dagger} U_{\text {Okubo }}^{\dagger} \Theta^{00}(0) U_{\text {Okubo }} U_{\eta} \eta\right| \beta\right\rangle .
$$

So we see that both for the boost and Hamiltonian, we need to calculate the matrix elements

$$
\left\langle\alpha\left|\eta U_{\eta}^{\dagger} U_{\text {Okubo }}^{\dagger} \Theta^{00}(0) U_{\text {Okubo }} U_{\eta} \eta\right| \beta\right\rangle \text {. }
$$

The only difference between the Hamiltonian and the boost is that the Hamiltonian matrix element is given by (2.111) multiplied with the momentum-conserving delta function, while the boost matrix element is given by (2.111) multiplied with a derivative of the delta function. A particularly convenient way to calculate this matrix element is by introducing a coupling with an external source $\rho(x)$ in the original chiral pion-nucleon Hamiltonian via

$$
H_{\rho}=\left.\int d^{3} x \rho(\vec{x}) \Theta^{00}(x)\right|_{x_{0}=0} .
$$

Taking the Fourier transform

$$
\rho(\vec{x})=\int d^{3} q \exp (i \vec{q} \cdot \vec{x}) \tilde{\rho}(\vec{q})
$$

we can calculate

$$
\frac{\delta H_{\rho}}{\delta \tilde{\rho}(\vec{q})}=\left.\int d^{3} x \exp (i \vec{q} \cdot \vec{x}) \Theta^{00}(x)\right|_{x_{0}=0} .
$$

After application of a unitary transformation to this operator, we obtain the desired matrix element by integrating over $\vec{q}$ :

$$
\int \frac{d^{3} q}{(2 \pi)^{3}}\left\langle\alpha\left|\eta U_{\eta}^{\dagger} U_{\text {Okubo }}^{\dagger} \frac{\delta H_{\rho}}{\delta \tilde{\rho}(\vec{q})} U_{\text {Okubo }} U_{\eta} \eta\right| \beta\right\rangle=\left\langle\alpha\left|\eta U_{\eta}^{\dagger} U_{\text {Okubo }}^{\dagger} \Theta^{00}(0) U_{\text {Okubo }} U_{\eta} \eta\right| \beta\right\rangle .
$$

For the calculation of the left-hand side, we proceed in a similar way as for the calculation of the current operator with an incoming momentum $\vec{q}$. Replacing finally the momentum transfer by

$$
\vec{q}=\vec{P}_{\alpha}-\vec{P}_{\beta},
$$

and dropping the momentum-conserving delta function (which is equivalent to performing an integration over $\vec{q}$ ) yields the right-hand side of Eq. (2.115). 


\section{H. Constraints on unitary phases}

The derived matrix elements for the current operators involve loop integrals which are ultraviolet divergent and need to be renormalized. This is carried out in the usual way by rewriting the bare LECs in terms of the renormalized ones and the corresponding counterterms, which have to cancel the ultraviolet (UV) divergences appearing in the loop integrals. Contrary to the scattering amplitude which is evaluated on shell, there is no guarantee that all UV divergences in the expressions for the nuclear potentials and exchange charge and current operators are cancelled by the counterterms. ${ }^{5}$ In fact, already the leading one-loop contributions to the $3 \mathrm{~N}$ force calculated by using the Okubo transformation cannot be renormalized [10]. Notice, however, that the parametrization of the unitary transformation in terms of the operator $\lambda A \eta$ corresponds to one particular choice of basis in the Fock space. The unitary ambiguity of the nuclear forces and currents can be systematically accounted for by invoking additional unitary transformations on the $\eta$-subspace of the Fock space. It was shown in [10] by introducing six additional unitary transformations, expressed in terms of continuously varying parameters $\alpha_{i}, i=1, \ldots 6$, that the renormalization of the $3 \mathrm{~N}$ force can be achieved for certain choices of the phases $\alpha_{i}$. Interestingly, the static part of the resulting nuclear potentials at order $Q^{4}$ was found to be independent on the unitary phases $\alpha_{i}$. On the other hand, the non-static contributions to the $2 \mathrm{~N}$ and $3 \mathrm{~N}$ forces at the same order do exhibit some degree of unitary ambiguity which manifests itself in the dependence on two (arbitrary) phases $\bar{\beta}_{8,9}$, see $[13,33]$ for more details and $[57,66]$ for an earlier discussion. Notice further that we had to invoke yet additional unitary transformations depending on the external electromagnetic source and leaving the results in the strong sector unaffected in order to renormalize the one-loop contributions to the one-pion exchange $2 \mathrm{~N}$ current operator [33]. Similarly, for the axial charge and current operators considered in this paper, we introduce all possible unitary transformations on the $\eta$-subspace of the Fock space, whose generators involve a single insertion of the external axial source. Clearly, such transformations do not affect the results we have obtained in the strong and electromagnetic sectors. In appendix B, we provide an explicit list of various unitary transformations which may contribute to the axial charge and current operators up to order $Q$ parametrized in terms of 33 phases $\alpha_{1, \ldots, 15}^{a x}, \alpha_{16}^{a x, \mathrm{LO}}$, $\alpha_{16}^{a x, \text { Static }}, \alpha_{16}^{a x, 1 / m}, \alpha_{16}^{a x}$,Tadpole,$\alpha_{17, \ldots, 30}^{a x}$ and an additional phase $\beta_{1}^{a x}$ related to the $d_{22}$-vertex with time derivative acting on the axial source as detailed in appendix C. The very high degree of unitary ambiguity as compared to the nuclear forces and electromagnetic currents at the same chiral order can be traced back to the appearance of the pion-axial-source interaction with $\kappa=-1$, which enters 30 out of the 33 generators listed in appendix B. Here and in what follows, we impose the following three conditions on the phases of the unitary transformations:

1. We require the one-loop contributions to the axial-vector current to be expressible in the form of 4-dimensional integrals with heavy-baryon propagators. This requirement is necessary for factorizability of the exchanged pions which itself is necessary (but not sufficient) for the renormalization of the axial-vector current. It leads to the following 14 constraints

$$
\begin{aligned}
\alpha_{6}^{a x} & =-\alpha_{4}^{a x}, \\
\alpha_{7}^{a x} & =-\alpha_{4}^{a x}, \\
\alpha_{8}^{a x} & =\alpha_{5}^{a x}, \\
\alpha_{9}^{a x} & =-\alpha_{4}^{a x}+\alpha_{5}^{a x}, \\
\alpha_{11}^{a x} & =\frac{1}{2}\left(\alpha_{4}^{a x}+\alpha_{5}^{a x}\right), \\
\alpha_{13}^{a x} & =\alpha_{4}^{a x}-\alpha_{5}^{a x}-\alpha_{7}^{a x}+\alpha_{10}^{a x}, \\
\alpha_{14}^{a x} & =\frac{1}{2}\left(\alpha_{4}^{a x}-\alpha_{5}^{a x}\right), \\
\alpha_{15}^{a x} & =-\alpha_{4}^{a x}-\alpha_{7}^{a x}+\alpha_{12}^{a x}, \\
\alpha_{20}^{a x} & =-\alpha_{17}^{a x}-\alpha_{18}^{a x}-\alpha_{19}^{a x}, \\
\alpha_{24}^{a x} & =-\alpha_{21}^{a x}-\alpha_{22}^{a x}-\alpha_{23}^{a x}, \\
\alpha_{27}^{a x} & =\alpha_{25}^{a x}, \\
\alpha_{28}^{a x} & =\alpha_{25}^{a x}-\alpha_{26}^{a x},
\end{aligned}
$$

\footnotetext{
${ }^{5}$ When evaluating the on-shell scattering amplitude, the remaining divergences have to cancel against the ones emerging from iterations of the dynamical equation.
} 


$$
\begin{aligned}
& \alpha_{29}^{a x}=\alpha_{25}^{a x}-\alpha_{26}^{a x}, \\
& \alpha_{30}^{a x}=-\alpha_{26}^{a x} .
\end{aligned}
$$

2. The resulting expressions for the axial charge and current operators are required to be properly renormalized. For the one-loop contributions to the one-pion exchange charge and current operators, this requires that all UV divergences are cancelled by expressing the relevant bare LECs $l_{i}$ from $\mathcal{L}_{\pi}^{(4)}$ and $d_{i}$ from $\mathcal{L}_{\pi N}^{(3)}$ in terms of the renormalized ones via

$$
\begin{aligned}
l_{i} & =l_{i}^{r}(\mu)+\gamma_{i} \lambda=: \frac{1}{16 \pi^{2}} \bar{l}_{i}+\gamma_{i} \lambda+\frac{\gamma_{i}}{16 \pi^{2}} \ln \left(\frac{M_{\pi}}{\mu}\right), \\
d_{i} & =d_{i}^{r}(\mu)+\frac{\beta_{i}}{F^{2}} \lambda=: \bar{d}_{i}+\frac{\beta_{i}}{F^{2}} \lambda+\frac{\beta_{i}}{16 \pi^{2} F^{2}} \ln \left(\frac{M_{\pi}}{\mu}\right),
\end{aligned}
$$

where $\mu$ is the scale introduced by dimensional regularization. Further, the quantity $\lambda$ is defined as

$$
\lambda=\frac{\mu^{d-4}}{16 \pi^{2}}\left(\frac{1}{d-4}+\frac{1}{2}\left(\gamma_{\mathrm{E}}-\ln 4 \pi-1\right)\right),
$$

with $\gamma_{\mathrm{E}}=-\Gamma^{\prime}(1) \simeq 0.577$ the Euler constant and $d$ the number of space-time dimensions. The $\beta$ - and $\gamma$ functions appearing in the above expressions are well known [58,67]. For the sake of completeness, we list below the expressions relevant to our calculations:

$$
\begin{aligned}
\gamma_{3} & =-\frac{1}{2}, \\
\gamma_{4} & =2, \\
\beta_{2}=-2 \beta_{5}=\frac{1}{2} \beta_{6} & =-\frac{1}{12}\left(1+5 g_{A}^{2}\right), \\
\beta_{15}=\beta_{18}=\beta_{22}=\beta_{23} & =0, \\
\beta_{16} & =\frac{1}{2} g_{A}+g_{A}^{3} .
\end{aligned}
$$

A cancellation of the UV divergences in the loop contributions to the single nucleon and to the one-pion exchange axial charge operators by the counterterms fixed by the $\beta$-functions listed above yields additional constraints on the unitary phases:

$$
\begin{gathered}
\alpha_{10}^{a x}+\alpha_{11}^{a x}=-\frac{1}{2}, \\
\alpha_{16}^{a x, \text { Tadpole }}=\alpha_{16}^{a x, \text { Static }}=\alpha_{16}^{a x, \mathrm{LO}}=-1 .
\end{gathered}
$$

A more general requirement of the cancellation of power-low divergences in addition to the logarithmic ones, which are taken care of in dimensional regularization, implies that the current should be renormalizable in $d=3$ dimensions. This yields an additional constraint

$$
\alpha_{1}^{a x}=0
$$

Thus, in total, we obtain 19 constraints from the requirement that the axial-vector current is renormalizable.

3. In addition to the renormalizability constraints specified above, we require the irreducible two- and three-nucleon pion-production amplitudes appearing in the expressions for the current operators to match the corresponding ones which appear in the expressions for the three- and four-nucleon forces, respectively, as visualized schematically earlier in Fig. 1. More precisely, we require the following matching condition between the one-pion exchange contributions to the nuclear forces and the corresponding axial current operator at the pion pole:

$$
\left.\lim _{k^{2} \rightarrow-M_{\pi}^{2}}\left(k^{2}+M_{\pi}^{2}\right) W\right|_{k^{2}=-M_{\pi}^{2}}=\lim _{k^{2} \rightarrow-M_{\pi}^{2}}\left(k^{2}+M_{\pi}^{2}\right) \tilde{A}_{\mu}^{b}(k) \tilde{a}^{\mu b}(k) .
$$

Here, $k \equiv|\vec{k}|$ refers to the momentum of the exchanged pion, while $k \cdot \tilde{a}^{b}(k)$ denotes the leading pion-nucleon vertex rather than the leading axial-source-pion interaction. This matching condition does not only represent a very natural choice of unitary phases as it makes the consistency between nuclear forces and current operators explicit, but is also expected to be advantageous from the practical point of view. In particular, it allows one to 
regularize the pion-pole contributions to the current operators in the way consistent with the regularization of the nuclear potentials. Notice further that in the actual calculation, the matching requirement, in combination with the renormalizability condition, leads to the vanishing of some pion-pole terms in the modified charge, i.e. in the quantity which enters the commutator on the left-hand side of Eq. (2.42),

$$
\tilde{\boldsymbol{A}}_{0}(\vec{k}, 0)-\frac{\partial}{\partial k_{0}} \vec{k} \cdot \overrightarrow{\tilde{\boldsymbol{A}}}\left(\vec{k}, k_{0}\right)+\frac{\partial}{\partial k_{0}}\left[W, \tilde{\boldsymbol{A}}\left(\vec{k}, k_{0}\right)\right]+m_{q} i \frac{\partial}{\partial k_{0}} \tilde{\boldsymbol{P}}\left(\vec{k}, k_{0}\right) .
$$

More precisely, we see that after the requirement of the renormalizability and matching to the $3 \mathrm{~N}$ force, the modified charge has no pion poles in the static limit. With the same constraints on the unitary phases, relativistic corrections to the modified charge have no second-order pion poles, i.e. no contributions proportional to $1 /\left(k^{2}+\right.$ $\left.M_{\pi}^{2}\right)^{2}$ (but do have simple poles). For this reasons, the continuity Eq. (2.42) simplifies for this choice of the unitary phases.

The required matching conditions lead to a number of constraints on the unitary phases which are summarized below. First, matching the two-pion-exchange two-nucleon current operator to the corresponding two-pion-onepion contributions to the three-nucleon force $(3 \mathrm{NF})$ at $\mathrm{N}^{3} \mathrm{LO}$ yields the three constraints

$$
\begin{aligned}
& \alpha_{10}^{a x}=1-2 \alpha_{4}^{a x}+\alpha_{5}^{a x}, \\
& \alpha_{26}^{a x}=1-\alpha_{25}^{a x} . \\
& \alpha_{12}^{a x}=0 .
\end{aligned}
$$

Next, matching the $1 / m$-corrections to the two-nucleon current involving the contact interactions with the corresponding $\mathrm{N}^{3} \mathrm{LO}$ three-nucleon forces gives an additional constraint

$$
\alpha_{23}^{a x}=1-\alpha_{21}^{a x}-\alpha_{22}^{a x} .
$$

Finally, matching the $1 / m$-corrections of the one-pion exchange axial-vector current with the corresponding $1 / m$-corrections to the $\mathrm{N}^{3} \mathrm{LO} 3 \mathrm{NF}$ gives two more constraints:

$$
\begin{aligned}
\alpha_{17}^{a x}+\alpha_{18}^{a x}+\alpha_{19}^{a x} & =\frac{1}{2}\left(1+2 \bar{\beta}_{8}\right), \\
\alpha_{16}^{1 / m} & =-\frac{1}{2}\left(1+2 \bar{\beta}_{9}\right) .
\end{aligned}
$$

As detailed in the previous sections, the expressions for the charge and current operators do, in general, involve contributions which depend on the energy transfer $k_{0}$ and arise from the explicit time dependence of the employed unitary transformations. In the Breit-frame with $k_{0}=0$, any choice of the non-locality for the nuclear force, which at $\mathrm{N}^{3} \mathrm{LO}$ is parametrized in terms of the phases $\bar{\beta}_{8}$ and $\bar{\beta}_{9}$, is consistent with the matching condition. In a general frame, one encounters pion-pole contributions to the axial-vector $2 \mathrm{~N}$ current operator proportional to the energy transfer $k_{0}$, which match the corresponding $1 / m$-corrections of the $3 \mathrm{NFs}$ only if one chooses

$$
\bar{\beta}_{8}=\frac{1}{2} \text {. }
$$

Similarly, the $k_{0} / m$-contributions to the single-nucleon current can be required to match the corresponding $1 / m$-corrections of one-pion exchange NN potential. This finally leads to

$$
\bar{\beta}_{9}=\frac{1}{2} \text {. }
$$

Note that with the last constraint, all $\alpha_{16}^{a x}$ phases are equal

$$
\alpha_{16}^{a x, \text { Tadpole }}=\alpha_{16}^{a x, \text { Static }}=\alpha_{16}^{a x, \mathrm{LO}}=\alpha_{16}^{a x, 1 / m}=-1 .
$$

In the following sections, we will show results for an arbitrary choice of $\bar{\beta}_{8}$ and $\bar{\beta}_{9}$. One should, however, keep in mind that the relativistic corrections to the axial current operator proportional to the energy transfer $k_{0}$ match the corresponding nuclear forces only for the particular choice of the phases $\bar{\beta}_{8}$ and $\bar{\beta}_{9}$ specified in Eqs. (2.128) and (2.129). Matching all remaining pion-pole contributions to the nuclear axial current operator, not explicitly mentioned above, with the corresponding terms in the nuclear potentials does not introduce any additional constraints on the phases. Here and in what follows, Eqs. (2.117), (2.121), (2.122), (2.125), (2.126) and (2.127) will be referred to as our standard choice of the unitary phases. 
With these constraints the currents have no further ambiguities (with respect to the considered class of unitary transformations), and the expressions become unique modulo the phases $\bar{\beta}_{8}$ and $\bar{\beta}_{9}$. Notice further that the continuity equation (2.42), being a manifestation of the symmetry, is valid independently on the choice of the unitary phases and thus does not lead to any constraints. Similarly, Eq. (2.77) represents a constraint on the axial current operator due to Poincaré invariance and is valid for all choices of the unitary phases, too. On the other hand, one may ask whether the unitary phases can be chosen in such a way, that Eq. (2.77) remains valid off the energy shell, i.e. the operator equation (2.78) holds true for $\boldsymbol{X}_{\mu}=0$. While we found this indeed to be possible, the resulting constraints on the unitary phases appear to be incompatible with the renormalizability requirement. Both Eqs. (2.42) and (2.77) represent extremely useful tools to check the expressions for the derived operators. We have explicitly verified that these equations are satisfied for the axial charge and current operators we have derived, see section X for a detailed discussion of the continuity equation.

\section{NOTATION FOR THE CURRENT OPERATORS}

Throughout this work, we employ the standard nuclear physics convention based on the nonrelativistic normalization for the nucleon states

$$
\left\langle\vec{p}^{\prime} \mid \vec{p}\right\rangle=\delta\left(\vec{p}^{\prime}-\vec{p}\right)
$$

In relativistic quantum field theory calculations, one usually uses

$$
\left\langle p^{\prime} \mid p\right\rangle=(2 \pi)^{3} 2 E_{p} \delta\left(\vec{p}^{\prime}-\vec{p}\right),
$$

with the nucleon energy given by

$$
E_{p}=\sqrt{\vec{p}^{2}+m^{2}}
$$

and factorizes the momentum conservation term $(2 \pi)^{3} \delta$ (sum of incoming momenta) out of the connected contributions to the scattering amplitude. Using the normalization as given in Eq. (3.1), it is convenient to factorize the factors of $(2 \pi)^{-3 A+3}$ out of the expressions for $A$-body operators. Specifically, we define

$$
\begin{aligned}
&\left\langle\vec{p}_{1}^{\prime}\left|\hat{A}_{2 \mathrm{~N}}^{\mu, a}\right| \vec{p}_{1}\right\rangle=: \delta\left(\vec{p}_{1}^{\prime}-\vec{p}_{1}-\vec{k}\right) A_{1 \mathrm{~N}}^{\mu, a}, \\
&\left\langle\vec{p}_{1}^{\prime} \vec{p}_{2}^{\prime}\left|\hat{A}_{2 \mathrm{~N}}^{\mu, a}\right| \vec{p}_{1} \vec{p}_{2}\right\rangle=:(2 \pi)^{-3} \delta\left(\vec{p}_{1}^{\prime}+\vec{p}_{2}^{\prime}-\vec{p}_{1}-\vec{p}_{2}-\vec{k}\right) A_{2 \mathrm{~N}}^{\mu, a}, \\
&\left\langle\vec{p}_{1}^{\prime} \vec{p}_{2}^{\prime} \vec{p}_{3}^{\prime}\left|\hat{A}_{3 \mathrm{~N}}^{\mu, a}\right| \vec{p}_{1} \vec{p}_{2} \vec{p}_{3}\right\rangle=:(2 \pi)^{-6} \delta\left(\vec{p}_{1}^{\prime}+\vec{p}_{2}^{\prime}+\vec{p}_{3}^{\prime}-\vec{p}_{1}-\vec{p}_{2}-\vec{p}_{3}-\vec{k}\right) A_{3 \mathrm{~N}}^{\mu, a},
\end{aligned}
$$

where $\vec{p}_{i}\left(\vec{p}_{i}^{\prime}\right)$ denotes the incoming (outgoing) momentum of nucleon $i, \vec{k}$ is the momentum of the external axial source while $a$ is an isospin index. Further, $\hat{\boldsymbol{A}}_{\mathrm{nN}}^{\mu}$ means that $\boldsymbol{A}_{\mathrm{nN}}^{\mu}$ is to be understood as an $n$-nucleon operator. Notice that the phase factors of $1 / \sqrt{2 E_{p}}$ for every nucleon field with momentum $p$ are kept in the expressions for the currents $\boldsymbol{A}^{\mu}$. This is particularly important for the calculation of $1 / \mathrm{m}^{2}$-corrections to the single-nucleon current. Here and in what follows, we suppress the " " over $\boldsymbol{A}^{\mu}$ to simplify the notation. It should be understood that all expressions for the axial charge and current operators given in the next sections are defined in momentum space according to Eq. (2.38). We will also use the same notation for the pseudoscalar current operators $\boldsymbol{P}_{\mathrm{nN}}$. In the following, we derive the contributions to the single-, two- and three-nucleon operators $A_{1 \mathrm{~N}}^{\mu, a} \equiv\left(A_{1 \mathrm{~N}}^{0, a}, \vec{A}_{1 \mathrm{~N}}^{a}\right), A_{2 \mathrm{~N}}^{\mu, a} \equiv\left(A_{2 \mathrm{~N}}^{0, a}, \vec{A}_{2 \mathrm{~N}}^{a}\right)$ and $A_{3 \mathrm{~N}}^{\mu, a} \equiv\left(A_{3 \mathrm{~N}}^{0, a}, \vec{A}_{3 \mathrm{~N}}^{a}\right)$ up to order $Q$ in the chiral expansion.

Last but not least, it is important to specify the dynamical equation since it affects the form of the relativistic corrections to both the current operators and nuclear forces [57]. Here and in what follows, we employ the $A$-nucleon Schrödinger equation with the relativistic expression for the kinetic-energy term, i.e.: ${ }^{6}$

$$
\left[\sum_{i}\left(\sqrt{p_{i}^{2}+m_{i}^{2}}-m_{i}\right)+V\right] \Psi=E \Psi,
$$

\footnotetext{
${ }^{6}$ Using the relativistic expression for the kinetic energy instead of its $1 / m$-expanded form is a matter of practical convenience. In the power counting scheme we employ, the standard nonrelativistic approximation of the kinetic energy is valid up-to-and-including $\mathrm{N}^{2} \mathrm{LO}$, i.e. order- $Q^{3}$ relative to the dominant terms in the nuclear force and current operator.
} 


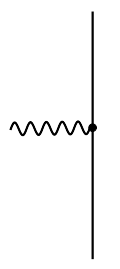

(1)

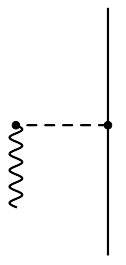

(2)

FIG. 2: Diagrams generating the lowest-order contribution $\overrightarrow{\boldsymbol{A}}_{1 \mathrm{~N}}^{\left(Q^{-3}\right)}$. Diagram (2) also contributes to $\boldsymbol{A}_{1 \mathrm{~N}}^{0\left(Q^{-1}\right)}$ as explained in the text. Solid dots denote vertices from $\mathcal{L}_{\pi N}^{(1)}$. Diagrams resulting from the application of the time reversal operation are not shown. For remaining notation see Fig. 1.

where $p_{i} \equiv\left|\vec{p}_{i}\right|$ and $m_{i}$ are the three-momentum and mass of the nucleon $i$. Notice that in the two-nucleon sector, it is customary to rewrite this expression in a form which resembles that of the usual nonrelativistic equation. This, in fact, is the convention adopted in the new chiral potentials of Refs. [5, 21]. Here and in what follows, we refrain from using this convention since we will also consider three-nucleon operators.

\section{SINGLE-NUCLEON AXIAL CHARGE AND CURRENT OPERATORS}

We now turn to the derivation of the contributions to the nuclear axial charge and current operators using the MUT and discuss the various classes of the contributions grouped according to the number of nucleons involved and the range of the interaction. To facilitate a comparison with calculations using different methods, we will explicitly indicate the dependence of the various terms upon the unitary phases, even though our final expressions are only given for the standard choice corresponding to Eqs. (2.117), (2.121), (2.122), (2.125), (2.126) and (2.127).

The first contribution to the single-nucleon axial vector current appears at order $Q^{-3}$ from the well-known diagrams shown in Fig. 2. The chiral order of the corresponding contributions can be read off from Eq. (2.12) by noticing that the leading-order $a_{\mu} N N, a_{\mu} \pi$ and $\pi N N$ vertices have, according to Eq. (2.10), the dimensions of $\kappa=0, \kappa=-1$ and $\kappa=1$, respectively. While there is no dependence on unitary phases at order $Q^{-3}$, diagram (2) of Fig. 2 also generates a contribution to the charge operator at order $Q^{-1}$ :

$$
(2) \sim k_{0} \frac{\alpha_{16}^{a x, \mathrm{LO}}}{k^{2}+M_{\pi}^{2}} .
$$

Here and in what follows, $\sim X$ means that the corresponding expressions involve terms proportional to $X$. If $k_{0}$ would be counted as a quantity of order $Q$, the contribution of this diagram would appear already at leading order. However, on shell, we associate $k_{0}$ with the difference of kinetic energies such that it counts as order $Q^{3}$ in our scheme. Thus, diagram (2) starts to contribute at order $Q^{-1}$. The well-known leading-order result for the axial charge and current operators has the form

$$
\begin{aligned}
& A_{1 \mathrm{~N}: \text { static }}^{0, a\left(Q^{-3}\right)}=0, \\
& \vec{A}_{1 \mathrm{~N}: \text { static }}^{a\left(Q^{-3}\right)}=-\frac{g_{A}}{2} \tau_{i}^{a}\left(\vec{\sigma}_{i}-\frac{\vec{k} \vec{k} \cdot \vec{\sigma}_{i}}{k^{2}+M_{\pi}^{2}}\right) .
\end{aligned}
$$

Notice that in the notation we are using, the chiral dimension of an $n$-nucleon contribution to the current operator can be easily read off from the corresponding expression by simply counting the powers of the soft scale (i.e. the three-momenta of the nucleons and external sources and $M_{\pi}$ ) and adding to the resulting dimension the factor of $3(n-2)$ to account for the different normalization of $n$-nucleon states.

There are only vanishing contributions at order $Q^{-2}$ :

$$
A_{1 \mathrm{~N}: \text { static }}^{0, a\left(Q^{-2}\right)}=0, \quad \vec{A}_{1 \mathrm{~N}: \text { static }}^{a\left(Q^{-2}\right)}=0 .
$$




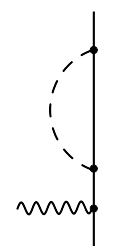

(1)

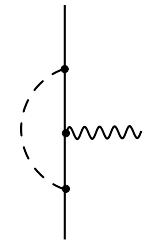

(2)

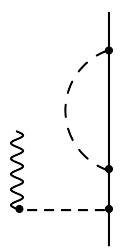

(3)

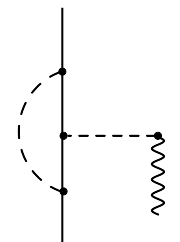

(4)

FIG. 3: One-loop diagrams which yield non-vanishing contributions to $\overrightarrow{\boldsymbol{A}}_{1 \mathrm{~N}}^{\left(Q^{-1}\right)}$ and $\boldsymbol{A}_{1 \mathrm{~N}}^{0(Q)}$. For notation see Fig. 2.

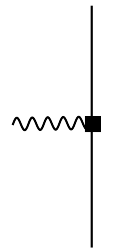

(1)

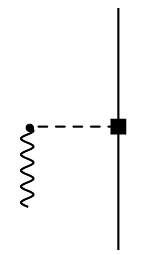

(2)

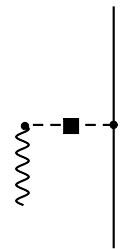

(3)

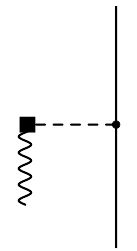

(4)

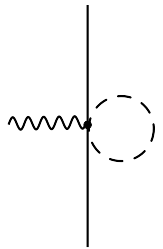

(5)

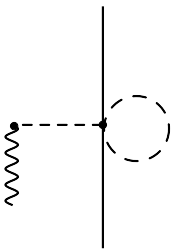

(6)

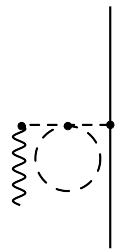

(7)

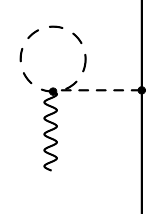

(8)

FIG. 4: Tree-level and tadpole diagrams yielding non-vanishing contributions to $\boldsymbol{A}_{1 \mathrm{~N}}^{\mu\left(Q^{-1}\right)}$. Solid dots (filled squares) denote vertices from $\mathcal{L}_{\pi N}^{(1)}$ or $\mathcal{L}_{\pi \pi}^{(2)}\left(\mathcal{L}_{\pi N}^{(3)}\right.$ or $\left.\mathcal{L}_{\pi \pi}^{(4)}\right)$. For remaining notation see Fig. 2.

At order $Q^{-1}$, we encounter three kinds of corrections. First, as already mentioned, there are terms emerging from the time-dependence of unitary transformations in diagram (2) of Fig. 2, which have the form

$$
\begin{aligned}
A_{1 \mathrm{~N}: \mathrm{UT}^{\prime}}^{0, a\left(Q^{-1}\right)} & =\frac{g_{A}}{2} \frac{k_{0}}{k^{2}+M_{\pi}^{2}} \vec{k} \cdot \vec{\sigma}_{i} \tau_{i}^{a}, \\
\vec{A}_{1 \mathrm{~N}: \mathrm{UT}^{\prime}}^{a\left(Q^{-1}\right)} & =0 .
\end{aligned}
$$

Secondly, we encounter the leading one-loop contributions together with tree-level diagrams with a single insertion of the $\mathcal{L}_{\pi N}^{(3)}$ vertices. There are no loop contributions to the charge operator at this order. More precisely, the leading one-loop contributions to the charge operator turn out to be proportional to the energy transfer and are thus shifted to order $Q$. In Fig. 3, we show the non-vanishing loop diagrams that contribute to the current (charge) operator at order $Q^{-1}(Q)$. The explicit dependence on the unitary phases for the charge operator is given by

$$
(3),(4) \sim k_{0} \frac{\alpha_{10}^{a x}+\alpha_{11}^{a x}-\alpha_{13}^{a x}}{k^{2}+M_{\pi}^{2}} .
$$

One-loop contributions to the current operator are, on the other hand, independent of the unitary phases. Further, in Fig. 4, we show tree-level and tadpole diagrams which generate nonvanishing contributions to the current operator, which turn out to be independent on the unitary phases. The final result for the charge and current operators at order $Q^{-1}$ in the static limit is given by

$$
\begin{aligned}
& A_{1 \mathrm{~N}: \text { static }}^{0, a\left(Q^{-1}\right)}=0 \\
& \vec{A}_{1 \mathrm{~N}: \text { static }}^{a\left(Q^{-1}\right)}=\frac{1}{2} \bar{d}_{22}\left(\vec{\sigma}_{i} k^{2}-\vec{k} \vec{k} \cdot \vec{\sigma}_{i}\right) \tau_{i}^{a}-\bar{d}_{18} M_{\pi}^{2} \tau_{i}^{a} \frac{\vec{k} \vec{k} \cdot \vec{\sigma}_{i}}{k^{2}+M_{\pi}^{2}} .
\end{aligned}
$$

Finally, we have to take into account the leading relativistic $1 / m$-corrections which in our counting scheme start contributing at order $Q^{-1}$. The corresponding diagrams are shown in Fig. 5. Diagram (1) contributes only to the charge operator at order $Q^{-1}$. On the other hand, diagram (2) contributes to the charge at order $Q^{-1}$ but also yields a correction to the current operator which is proportional to $k_{0}$ and, therefore, contributes at order $Q$. We find the 


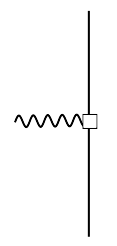

(1)

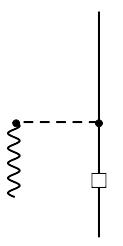

(2)

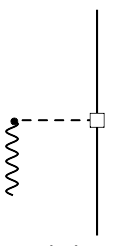

(3)

FIG. 5: Relativistic $1 / m$-contributions to $\boldsymbol{A}_{1 \mathrm{~N}}^{0\left(Q^{-1}\right)}$ and $\overrightarrow{\boldsymbol{A}}_{1 \mathrm{~N}}^{(Q)}$. Solid dots (open rectangles) denote vertices from $\mathcal{L}_{\pi N}^{(1)}(1 / m-$ corrections from $\mathcal{L}_{\pi N}^{(2)}$ ). For remaining notation see Fig. 2.

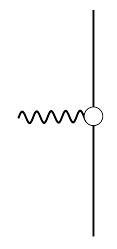

(1)

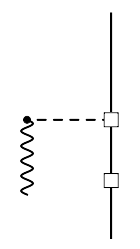

(2)

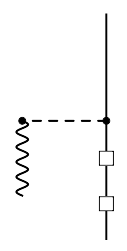

(3)<smiles>CCC(OC)OC</smiles>

(4)

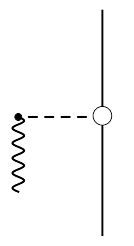

(5)

FIG. 6: Relativistic $1 / m^{2}$-contributions to $\overrightarrow{\boldsymbol{A}}_{1 \mathrm{~N}}^{(Q)}$. Solid dots (open rectangles) denote vertices from $\mathcal{L}_{\pi N}^{(1)}(1 / m$-corrections from $\left.\mathcal{L}_{\pi N}^{(2)}\right)$. Open circle denotes $1 / m^{2}$-corrections from $\mathcal{L}_{\pi N}^{(3)}$. For remaining notation see Fig. 2.

following dependence of the resulting contributions on the unitary phases for the charge

$$
\text { (2) } \sim \frac{1+\alpha_{16}^{a x, \mathrm{LO}}}{k^{2}+M_{\pi}^{2}},
$$

and current operator

$$
\begin{aligned}
& (2) \sim \frac{k_{0}}{m} \frac{\alpha_{17}^{a x}+\alpha_{18}^{a x}+\alpha_{19}^{a x}}{\left(k^{2}+M_{\pi}^{2}\right)^{2}}, \\
& (3) \sim \frac{k_{0}}{m} \frac{\alpha_{16}^{a x, 1 / m}}{k^{2}+M_{\pi}^{2}} .
\end{aligned}
$$

The final result for the $1 / m$ corrections to the $1 \mathrm{~N}$ axial-vector current for our standard choice of unitary phases reads

$$
\begin{aligned}
A_{1 \mathrm{~N}: 1 / m}^{0, a\left(Q^{-1}\right)} & =-\frac{g_{A}}{2 m} \tau_{i}^{a} \vec{\sigma}_{i} \cdot \vec{k}_{i}, \\
\vec{A}_{1 \mathrm{~N}: 1 / \mathrm{m}}^{a\left(Q^{-1}\right)} & =0,
\end{aligned}
$$

where

$$
\vec{k}=\vec{p}_{i}^{\prime}-\vec{p}_{i}, \quad \vec{k}_{i}=\frac{\vec{p}_{i}^{\prime}+\vec{p}_{i}}{2}
$$

This completes the derivation of the $1 \mathrm{~N}$ terms at order $Q^{-1}$.

There are no corrections to the $1 \mathrm{~N}$ charge and current operators at order $Q^{0}$. In particular, the absence of $c_{i} / m$ corrections and $k_{0}$-dependent contributions can be understood from the fact that there are no order- $Q^{-2}$ terms while the contributions of one-loop diagrams with a single insertion of subleading interactions vanish after renormalization. 
Finally, there are various contributions at $\operatorname{order} Q$. We begin with terms proportional to $k_{0} / m$ which emerge from diagrams (2) and (3) of Fig. 5. Their dependence on unitary phases is given by Eq. (4.9). For the standard choice of unitary phases, the explicit expressions have the form

$$
\begin{aligned}
& A_{1 \mathrm{~N}: 1 / m, \mathrm{UT}^{\prime}}^{0, a(Q)}=0 \\
& \vec{A}_{1 \mathrm{~N}: 1 / m, \mathrm{UT}^{\prime}}^{a(Q)}=-\frac{g_{A} k_{0}}{8 m} \frac{\vec{k}}{k^{2}+M_{\pi}^{2}} \tau_{i}^{a}\left(2\left(1+2 \bar{\beta}_{9}\right) \vec{\sigma}_{i} \cdot \vec{k}_{i}-\left(1+2 \bar{\beta}_{8}\right) \vec{k} \cdot \vec{\sigma}_{i} \frac{p_{i}^{\prime 2}-p_{i}^{2}}{k^{2}+M_{\pi}^{2}}\right) .
\end{aligned}
$$

For the static part which is proportional to $k_{0}$, we get nonvanishing contributions from diagrams (3) and (4) of Fig. 3 as well as from the diagrams shown in Fig. 4. For the standard choice of unitary phases we obtain after renormalization

$$
\begin{aligned}
& A_{1 \mathrm{~N}: \text { static, } \mathrm{UT}^{\prime}}^{0, a(Q)}=-k_{0} \frac{\tau_{i}}{2} \vec{k} \cdot \vec{\sigma}_{i}\left[\bar{d}_{22}+\frac{2 \bar{d}_{18} M_{\pi}^{2}}{k^{2}+M_{\pi}^{2}}\right], \\
& \vec{A}_{1 \mathrm{~N}: \text { static, } \mathrm{UT}^{\prime}}^{a(Q)}=0 .
\end{aligned}
$$

So far, the $k_{0}$ dependence in our expressions emerged entirely from the time dependence of the unitary transformations. The contribution proportional to $\bar{d}_{22}$ in Eq. (4.14), however, originates directly from the interaction term

$$
-d_{22} N^{\dagger} S_{\mu}\left(\partial^{2} \boldsymbol{a}^{\mu}-\partial^{\mu} \partial_{\nu} \boldsymbol{a}^{\nu}\right) \cdot \boldsymbol{\tau} N=d_{22} N^{\dagger} S_{\mu}\left(\partial^{\mu} \dot{\boldsymbol{a}}^{0}\right) \cdot \boldsymbol{\tau} N+\ldots
$$

of the Lagrangian $\mathcal{L}_{\pi N}^{(3)}$ when evaluating the corresponding Feynman diagram. In the formulation presented so far, we have not included time-derivatives of the axial vector source in the interaction. Rather, their time-derivatives were eliminated from the action by performing partial integration in time. In Appendix C, we show that the term $\propto \bar{d}_{22}$ indeed emerges from a corresponding unitary transformations if the time-derivative of the axial source is eliminated via partial time-integration.

The second class of order- $Q$ contributions involves relativistic $1 / m^{2}$-corrections. The corresponding non-vanishing diagrams are visualized in Fig. 6. They contribute only to the current operator. The dependence on the unitary phases of the contributions from diagrams in Fig. 6 is given by

$$
\begin{aligned}
(2) & \sim 1+\alpha_{16}^{a x, 1 / m}, \\
(3),(4) & \sim 1-\alpha_{17}^{a x}-\alpha_{18}^{a x}-\alpha_{19}^{a x},
\end{aligned}
$$

while diagrams (1) and (5) do not depend on unitary phases. Our final result for $1 / \mathrm{m}^{2}$-corrections at order $Q$ reads

$$
\begin{aligned}
A_{1 \mathrm{~N}: 1 / m^{2}}^{0, a(Q)} & =0 \\
\vec{A}_{1 \mathrm{~N}: 1 / \mathrm{m}^{2}}^{a(Q)} & =\frac{g_{A}}{16 m^{2}} \tau_{i}^{a}\left(\vec{k} \vec{k} \cdot \vec{\sigma}_{i}\left(1-2 \bar{\beta}_{8}\right) \frac{\left(p_{i}^{\prime 2}-p_{i}^{2}\right)^{2}}{\left(k^{2}+M_{\pi}^{2}\right)^{2}}-2 \vec{k} \frac{\left(p_{i}^{\prime 2}+p_{i}^{2}\right) \vec{k} \cdot \vec{\sigma}_{i}-2 \bar{\beta}_{9}\left(p_{i}^{\prime 2}-p_{i}^{2}\right) \vec{k}_{i} \cdot \vec{\sigma}_{i}}{k^{2}+M_{\pi}^{2}}\right. \\
& \left.+2 i\left[\vec{k} \times \vec{k}_{i}\right]+\vec{k} \vec{k} \cdot \vec{\sigma}_{i}-4 \vec{k}_{i} \vec{k}_{i} \cdot \vec{\sigma}_{i}+\vec{\sigma}_{i}\left(2\left(p_{i}^{\prime 2}+p_{i}^{2}\right)-k^{2}\right)\right) .
\end{aligned}
$$

In order to obtain this result, we have calculated the contributions of all diagrams in Fig. 6 using the MUT. Notice that we have also included in Eq. (4.18) the corrections accounting for the nonrelativistic normalization of the nucleon fields which amounts to multiplying the expressions for the current operator with the factors of $\sqrt{m / E_{p}}$ for every external nucleon with a momentum $p$, see $[21,57]$ for details. Expanding the result in $1 / m$ we obtain, in addition to the expressions for diagrams of Fig. 6, the contribution

$$
\delta A_{1 \mathrm{~N}: 1 / m^{2}}^{\mu, a,(Q)}=\sqrt{\frac{m}{E_{p_{i}^{\prime}}}} A_{1 \mathrm{~N}: \text { static }}^{\mu, a,\left(Q^{-3}\right)} \sqrt{\frac{m}{E_{p_{i}}}}-A_{1 \mathrm{~N}: \text { static }}^{\mu, a,\left(Q^{-3}\right)}=-\frac{p_{i}^{\prime 2}+p_{i}^{2}}{4 m^{2}} A_{1 \mathrm{~N}: \text { static }}^{\mu, a,\left(Q^{-3}\right)}+\mathcal{O}\left(1 / m^{4}\right),
$$

which is already accounted for in Eq. (4.18).

The third kind of order- $Q$ contributions emerges from relativistic $1 / m$-corrections to the leading one-loop terms. The corresponding non-vanishing diagrams are shown in Figs. 7, 8 and 9. The dependence on the unitary phases has the form given by

$$
(2) \sim 1+\alpha_{16}^{a x, \text { Static }},
$$




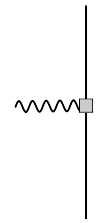

(1)

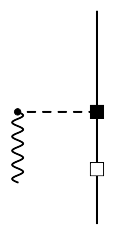

(2)

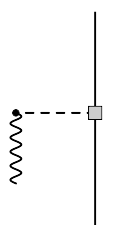

(3)

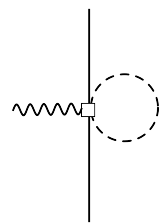

(4)

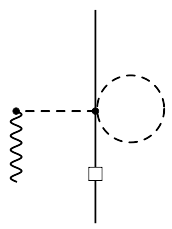

(5)

FIG. 7: Relativistic $1 / m$-contributions to $\boldsymbol{A}_{1 \mathrm{~N}}^{\mu(Q)}$ from tree-level and tadpole diagrams. Solid dots (open rectangles) denote vertices from $\mathcal{L}_{\pi N}^{(1)}\left(1 / m\right.$-corrections from $\left.\mathcal{L}_{\pi N}^{(2)}\right)$. Shaded rectangles denote $1 / m$-corrections from $\mathcal{L}_{\pi N}^{(3)}$. Black rectangles denote vertices from $\mathcal{L}_{\pi N}^{(3)}$ in the static limit. For remaining notation see Fig. 2.

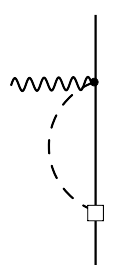

(6)

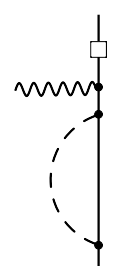

(13)

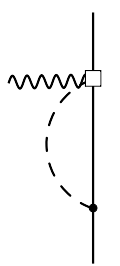

(7)

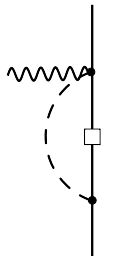

(8)

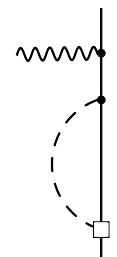

(9)

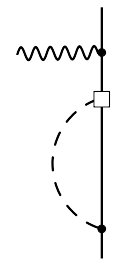

(10)

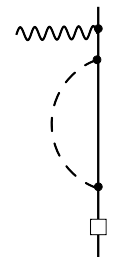

(11)

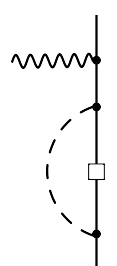

(12)

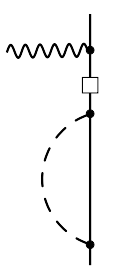

(14)
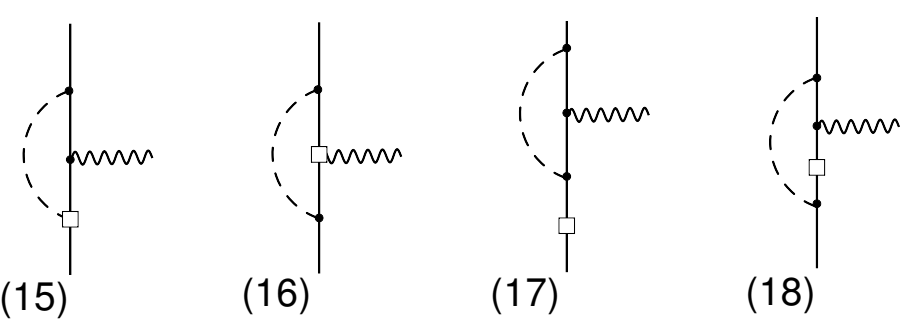

FIG. 8: Non-pion-pole relativistic $1 / m$-contributions to $\boldsymbol{A}_{1 \mathrm{~N}}^{\mu(Q)}$ from one-loop diagrams. Solid dots (open rectangles) denote vertices from $\mathcal{L}_{\pi N}^{(1)}\left(1 / m\right.$-corrections from $\left.\mathcal{L}_{\pi N}^{(2)}\right)$. For remaining notation see Fig. 2.

$$
\begin{aligned}
(5) & \sim 1+\alpha_{16}^{a x, \text { Tadpole }}, \\
(21),(22),(25),(27) & \sim 1+\alpha_{16}^{a x, \mathrm{LO}}, \\
(24) & \sim 6+2\left(\alpha_{10}^{a x}+\alpha_{11}^{a x}-\alpha_{13}^{a x}\right)+3 \alpha_{16}^{a x, \mathrm{LO}}, \\
(26),(29) & \sim 3+2\left(\alpha_{10}^{a x}+\alpha_{11}^{a x}-\alpha_{13}^{a x}\right),
\end{aligned}
$$

for the charge operator and

$$
\begin{aligned}
(9),(21) & \sim 1+2 \bar{\beta}_{9} \\
(10),(22) & \sim 1-2 \bar{\beta}_{9}, \\
(11) & \sim 1+2 \bar{\beta}_{8}+2 \alpha_{1}^{a x} \\
(13) & \sim \alpha_{1}^{a x} \\
(14) & \sim 1-2 \bar{\beta}_{8} \\
(23) & \sim 1+\alpha_{16}^{a x, 1 / m} \\
(24) & \sim\left(1+2 \bar{\beta}_{8}-2 \alpha_{12}^{a x}\right) \frac{1}{k^{2}+M_{\pi}^{2}}[\ldots]+\left(1-\alpha_{13}^{a x}\right) \frac{1}{\left(k^{2}+M_{\pi}^{2}\right)^{2}}[\ldots], \\
(26) & \sim \alpha_{12}^{a x} \frac{1}{k^{2}+M_{\pi}^{2}}[\ldots]+\left(2-\alpha_{13}^{a x}-\alpha_{17}^{a x}-\alpha_{18}^{a x}-\alpha_{19}^{a x}\right) \frac{1}{\left(k^{2}+M_{\pi}^{2}\right)^{2}}[\ldots],
\end{aligned}
$$




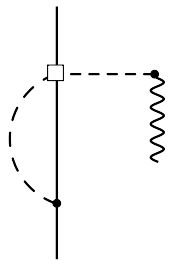

(19)

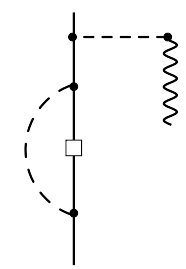

(25)

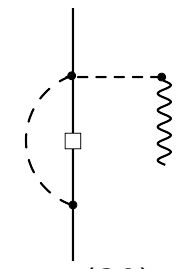

(20)

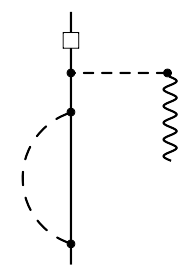

(26)

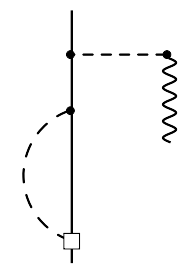

(21)

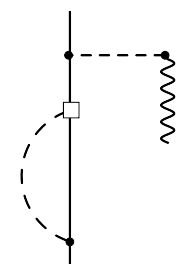

(22)

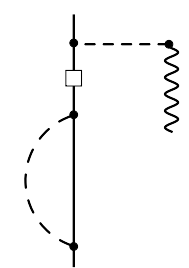

(27)

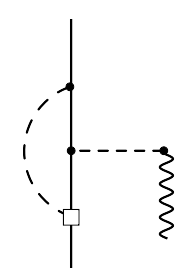

(28)

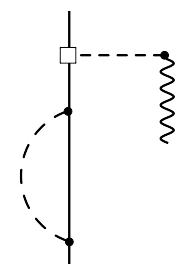

(23)

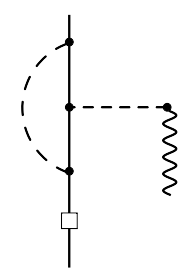

(29)

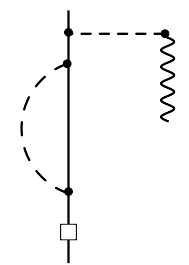

(24)

FIG. 9: Pion-pole relativistic $1 / m$-contributions to $\boldsymbol{A}_{1 \mathrm{~N}}^{\mu(Q)}$ from one-loop diagrams. Solid dots (open rectangles) denote vertices from $\mathcal{L}_{\pi N}^{(1)}\left(1 / m\right.$-corrections from $\left.\mathcal{L}_{\pi N}^{(2)}\right)$. For remaining notation see Fig. 2.

$$
(27) \sim\left(1-2 \bar{\beta}_{8}\right) \frac{1}{k^{2}+M_{\pi}^{2}}[\ldots]+\left(1-\alpha_{17}^{a x}-\alpha_{18}^{a x}-\alpha_{19}^{a x}\right) \frac{1}{\left(k^{2}+M_{\pi}^{2}\right)^{2}}[\cdots]
$$

for the current operators, where $[\ldots]$ denote spin-isospin-momentum structures which do not depend on the unitary phases. All other diagrams do not induce any dependence on the unitary phases. For the standard choice of the phases we obtain the following simple result after renormalization:

$$
\begin{aligned}
& A_{1 \mathrm{~N}: 1 / m}^{0, a)}=\vec{d}_{22} \vec{k}_{i} \cdot \vec{\sigma}_{i} \tau_{i}^{a} \frac{k^{2}}{2 m}, \\
& \vec{A}_{1 \mathrm{~N}: 1 / \mathrm{m}}^{a(Q)}=0 .
\end{aligned}
$$

Before discussing the final class of corrections at order $Q$ corresponding to static contributions at two-loop level, it is instructive to compare our results with the on-shell expressions for the three-point function with an external axial-vector source, which can be parametrized in terms of the form factors $G_{A}(t)$ and $G_{P}(t)$. The relativistic parametrization is given by

$$
\left\langle N\left(p_{i}^{\prime}\right)\left|A^{\mu, a}(0)\right| N\left(p_{i}\right)\right\rangle=\frac{1}{2 m} \bar{u}\left(p_{i}^{\prime}\right)\left[\gamma^{\mu} \gamma_{5} G_{A}(t)+\frac{k^{\mu}}{2 m} \gamma_{5} G_{P}(t)\right] \frac{\tau_{i}^{a}}{2} u\left(p_{i}\right),
$$

where

$$
t=k_{0}^{2}-k^{2}, \quad(\text { with } k \equiv|\vec{k}|)
$$

and the spinors are normalized as

$$
\bar{u}\left(p_{i}\right) u\left(p_{i}\right)=2 m .
$$

The chiral expansion of the form factors can be found e.g. in [68, 69], see also [70, 71] for results obtained within Lorentz-invariant formulations. Rewritten in our notation, the chiral expansion of the axial form factor is given by

$$
G_{A}(t)=g_{A}+\left(\bar{d}_{22}+f_{0}^{A} M_{\pi}^{2}\right) t+f_{1}^{A} t^{2}+G_{A}^{\left(Q^{4}\right)}(t)+\mathcal{O}\left(Q^{5}\right)
$$

where $f_{i}^{A}$ are LECs of dimension $\mathrm{GeV}^{-4}$ and

$$
G_{A}^{\left(Q^{4}\right)}(t)=\frac{t^{3}}{\pi} \int_{9 M_{\pi}^{2}}^{\infty} \frac{\operatorname{Im} G_{A}^{\left(Q^{4}\right)}\left(t^{\prime}\right)}{t^{\prime 3}\left(t^{\prime}-t-i \epsilon\right)} d t^{\prime}
$$


with the imaginary part calculated utilizing the Cutkosky rules [68]

$$
\begin{aligned}
\operatorname{Im} G_{A}^{\left(Q^{4}\right)}(t) & =\frac{g_{A}}{192 \pi^{3} F_{\pi}^{4}} \int_{z^{2}<1} d \omega_{1} d \omega_{2}\left[6 g_{A}^{2}\left(\sqrt{t} \omega_{1}-M_{\pi}^{2}\right)\left(\frac{l_{2}}{l_{1}}+z\right) \frac{\arccos (-z)}{\sqrt{1-z^{2}}}\right. \\
& \left.+2 g_{A}^{2}\left(M_{\pi}^{2}-\sqrt{t} \omega_{1}-\omega_{1}^{2}\right)+M_{\pi}^{2}-\sqrt{t} \omega_{1}+2 \omega_{1}^{2}\right]
\end{aligned}
$$

where

$$
\omega_{i}=\sqrt{l_{i}^{2}+M_{\pi}^{2}} \quad \text { with } \quad i=1,2, \quad \text { and } \quad z=\hat{l}_{1} \cdot \hat{l}_{2}=\frac{\omega_{1} \omega_{2}-\sqrt{t}\left(\omega_{1}+\omega_{2}\right)+\frac{1}{2}\left(t+M_{\pi}^{2}\right)}{l_{1} l_{2}}
$$

Here and in what follows, $l_{i} \equiv\left|\vec{l}_{i}\right|$, while $\hat{l}_{i} \equiv \vec{l}_{i} / l_{i}$. In the chiral limit, the expression for $\operatorname{Im} G_{A}^{\left(Q^{4}\right)}(t) \operatorname{simplifies}$ to

$$
\left.\operatorname{Im} G_{A}^{\left(Q^{4}\right)}(t)\right|_{M_{\pi}=0}=\frac{2 \stackrel{\circ}{g}_{A} t^{2}}{9(16 \pi)^{3} F^{4}}\left[\stackrel{\circ}{g}_{A}^{2}\left(\frac{64}{35} \pi^{2}+1\right)-1\right]
$$

where $\stackrel{\circ}{g}_{A}$ and $F$ are the axial coupling and pion decay constants in the chiral limit, respectively. Note that $\operatorname{Im} G_{A}^{\left(Q^{4}\right)}(t)$ grows at least quadratically in $t$ [68], and for this reason one has to introduce three subtractions in the dispersion integral of Eq. (4.28). The linear combination $\bar{d}_{22}+f_{0}^{A} M_{\pi}^{2}$ is related to the axial radius of the nucleon via

$$
\bar{d}_{22}+f_{0}^{A} M_{\pi}^{2}=\frac{g_{A}}{6}\left\langle r_{A}^{2}\right\rangle
$$

The LECs $f_{i}^{A}$ refer to the corresponding linear combinations of LEC's from $\mathcal{L}_{\pi N}^{(5)}$. Notice that there are no contributions to $f_{i}^{A}$ from $1 / m^{2}$ corrections since the latter emerge only from loop diagrams and for this reason start contributing at higher orders. Indeed, the tree-level relativistic corrections associated with diagram (1) of Fig. 2 are entirely given by the $1 / m$-expansion of $\bar{u}\left(p^{\prime}\right) \gamma^{\mu} \gamma_{5} u(p) / 2 m$ :

$$
\begin{aligned}
\bar{u}\left(p^{\prime}\right) \gamma^{0} \gamma_{5} u(p) / 2 m & =\frac{\vec{k}_{i} \cdot \vec{\sigma}_{i}}{m}+\mathcal{O}\left(m^{-3}\right), \\
\bar{u}\left(p^{\prime}\right) \gamma^{j} \gamma_{5} u(p) / 2 m & =\sigma_{i}^{j}-\frac{1}{16 m^{2}}\left[4 i\left[\vec{k} \times \vec{k}_{i}\right]^{j}+2 k^{j} \vec{k} \cdot \vec{\sigma}_{i}-\sigma^{j}\left(k^{2}+2\left(p_{i}^{\prime 2}+p_{i}^{2}-2 k_{i}^{2}\right)\right)-8 k_{i}^{j} \vec{k}_{i} \cdot \vec{\sigma}_{i}\right]+\mathcal{O}\left(m^{-4}\right) .
\end{aligned}
$$

The pseudoscalar form-factor is given up to order $Q^{4}$ by [72]

$$
G_{P}(t)=\frac{4 m g_{\pi N} F_{\pi}}{M_{\pi}^{2}-t}-\frac{2}{3} g_{A} m^{2}\left\langle r_{A}^{2}\right\rangle+m^{2} f_{1}^{P} t+m^{2} G_{P}^{\left(Q^{2}\right)}(t)+\mathcal{O}\left(Q^{3}\right)
$$

where $f_{i}^{P}$ denotes the corresponding linear combinations of the LECs of dimension $\mathrm{GeV}^{-4}$ from $\mathcal{L}_{\pi N}^{(5)}$ and

$$
G_{P}^{\left(Q^{2}\right)}(t)=\frac{t^{2}}{\pi} \int_{9 M_{\pi}^{2}}^{\infty} \frac{\operatorname{Im} G_{P}^{\left(Q^{2}\right)}\left(t^{\prime}\right)}{t^{\prime 2}\left(t^{\prime}-t-i \epsilon\right)} d t^{\prime}
$$

with the imaginary part calculated by the Cutkosky rules [72]

$$
\operatorname{Im} G_{P}^{\left(Q^{2}\right)}(t)=\operatorname{Im} G_{P}^{(1)}(t)+\operatorname{Im} G_{P}^{(2)}(t)
$$

and

$$
\begin{aligned}
\operatorname{Im} G_{P}^{(1)}(t) & =\frac{g_{A}}{8 \pi^{3} F_{\pi}^{4}} \int_{z^{2}<1} d \omega_{1} d \omega_{2}\left[\frac{1}{18}-\frac{M_{\pi}^{4}}{12\left(t-M_{\pi}^{2}\right)^{2}}+\frac{4 \omega_{1}^{2}-M_{\pi}^{2}}{6 t}+\frac{\omega_{1}^{2}\left(3 M_{\pi}^{2}-t\right)}{\left(t-M_{\pi}^{2}\right)^{2}}+\frac{2 M_{\pi}^{2} \omega_{1} \omega_{2} z}{t\left(t-M_{\pi}^{2}\right)} \frac{l_{2}}{l_{1}}\right] \\
\operatorname{Im} G_{P}^{(2)}(t) & =\frac{g_{A}^{3}}{8 \pi^{3} F_{\pi}^{4} t} \int_{z^{2}<1} d \omega_{1} d \omega_{2}\left[\left(M_{\pi}^{2}-\sqrt{t} \omega_{1}\right)\left(z+\frac{l_{2}}{l_{1}}\right) \frac{\arccos (-z)}{\sqrt{1-z^{2}}}+\frac{l_{1}^{2}}{3}+\frac{t}{9}\right. \\
& \left.+\frac{M_{\pi}^{2}}{t-M_{\pi}^{2}}\left(\frac{7}{8} \sqrt{t}-\omega_{1}-\omega_{2}\right)\left(2 \omega_{1} z \frac{l_{2}}{l_{1}}+\sqrt{t}+\left(\left(t+M_{\pi}^{2}\right)\left(4 \omega_{1}-\sqrt{t}\right)-4 \sqrt{t} \omega_{1} \omega_{2}\right) \frac{\arccos (-z)}{2 l_{1} l_{2} \sqrt{1-z^{2}}}\right)\right] .
\end{aligned}
$$


In the chiral limit, one finds

$$
\left.\operatorname{Im} G_{P}^{\left(Q^{2}\right)}(t)\right|_{M_{\pi}=0}=\frac{\stackrel{\circ}{g}_{A} t}{9(8 \pi)^{3} F^{4}}\left[1-\stackrel{\circ}{g}_{A}^{2}\left(1+\frac{64}{35} \pi^{2}\right)\right],
$$

where we have already used the Goldberger-Treiman relation. Due to linear growth of $\operatorname{Im} G_{P}^{\left(Q^{2}\right)}(t)$ in $t[72]$, one needs two subtractions in the dispersion integral of Eq. (4.35).

Similarly to the case of the axial form-factor, there are no $1 / m^{2}$-corrections in $f_{i}^{P}$. In a relativistic calculation, diagram (2) of Fig. 2 generates the contribution

$$
\frac{4 m g_{\pi N} F_{\pi}}{M_{\pi}^{2}-t}
$$

to the pseudoscalar form factor. The nonrelativistic expansion then emerges entirely from the expansion of $\bar{u}\left(p^{\prime}\right) \gamma_{5} u(p) / 2 m$ in inverse powers of the nucleon mass,

$$
\bar{u}\left(p^{\prime}\right) \gamma_{5} u(p) / 2 m=-\frac{\vec{k} \cdot \vec{\sigma}_{i}}{2 m}+\mathcal{O}\left(1 / m^{3}\right) .
$$

We are now in the position to compare our results for the single-nucleon axial-vector current operator with the on-shell results up-to-and-including the one-loop corrections. Notice that given the absence of the iterative contributions to the single-nucleon scattering amplitude, ${ }^{7}$ we do expect the derived expressions for the charge and current operator to match the three-point function with an external axial vector source on the energy shell. Using the Goldberger-Treiman discrepancy

$$
g_{\pi N}=\frac{g_{A} m}{F_{\pi}}\left(1-\frac{2 M_{\pi}^{2} \bar{d}_{18}}{g_{A}}\right),
$$

one observes that our results for the charge operator agree with the on-shell expressions even when the energyconserving delta-function is dropped. For the current operator, the results for the off-shell kinematics agree only up-to-and-including $1 / m$-corrections, while the disagreement starts first at the level of the $1 / m^{2}-$ and $k_{0} / m$-terms. The difference is given by

$$
\begin{aligned}
& {\left[\sqrt{\frac{m}{E_{p_{i}^{\prime}}}} \frac{1}{2 m} \bar{u}\left(p_{i}^{\prime}\right)\left[\vec{\gamma} \gamma_{5} G_{A}(t)+\frac{\vec{k}}{2 m} \gamma_{5} G_{P}(t)\right] \frac{\tau_{i}^{a}}{2} u\left(p_{i}\right) \sqrt{\frac{m}{E_{p_{i}}}}\right]_{1 / m^{2}-\text { and } k_{0} / m-\text { parts }}+\vec{A}_{1 \mathrm{~N}: 1 / m^{2}}^{a(Q)}+\vec{A}_{1 \mathrm{~N}: 1 / \mathrm{m}, \mathrm{UT}^{\prime}}^{a(Q)} } \\
= & -\left(k_{0}-\frac{p_{i}^{\prime}}{2 m}+\frac{p_{i}^{2}}{2 m}\right) \frac{g_{A}}{8 m} \frac{\vec{k}}{k^{2}+M_{\pi}^{2}} \tau_{i}^{a}\left[-\left(1+2 \bar{\beta}_{8}\right)\left(p_{i}^{\prime 2}-p_{i}^{2}\right) \frac{\vec{k} \cdot \vec{\sigma}_{i}}{k^{2}+M_{\pi}^{2}}+2\left(1+2 \bar{\beta}_{9}\right) \vec{k}_{i} \cdot \vec{\sigma}_{i}\right],
\end{aligned}
$$

which, given the on-shell relation $k_{0}={ }_{1}^{\prime 2} / 2 m-p_{i}^{2} / 2 m$, is indeed an off-the-energy-shell effect. Notice that the off-shell difference disappears for $\bar{\beta}_{8}=\bar{\beta}_{9}=-1 / 2$, which is, however, incompatible with the matching condition to the nuclear forces except for the Breit frame. It is remarkable that even the static terms proportional to $k_{0}$, which are parametrized by unitary phases and for this reason describe off-shell effects, agree with the on-shell result for our standard choice of the phases. In particular, if $\alpha_{16}^{a x, \mathrm{LO}}$ were not fixed, the leading contribution to the charge operator at order $Q^{-1}$ would read

$$
A_{1 \mathrm{~N}: \mathrm{UT}^{\prime}}^{0, a\left(Q^{-1}\right)}=-\alpha_{16}^{a x, \mathrm{LO}} \frac{g_{A}}{2} \frac{k_{0}}{k^{2}+M_{\pi}^{2}} \vec{k} \cdot \vec{\sigma}_{i} \tau_{i}^{a}
$$

On the other hand, the leading contribution to the on-shell charge comes from the pseudoscalar form factor given by

$$
-\frac{1}{2 m} \bar{u}\left(p_{i}^{\prime}\right) \frac{k^{0}}{2 m} \gamma_{5} G_{P}(t) \frac{\tau_{i}^{a}}{2} u\left(p_{i}\right)=\frac{g_{A}}{2} \frac{k_{0}}{k^{2}+M_{\pi}^{2}} \vec{k} \cdot \vec{\sigma}_{i} \tau_{i}^{a},
$$

\footnotetext{
7 It is important to keep in mind that diagrams like the ones shown in Fig. 6 can not be interpreted as iterations with the effective potential since the non-relativistic kinetic energy and its corrections at higher orders in the $1 / m$-expansion are not part of the potential, see Eq. (3.5).
} 
where we have used Eqs. (4.40), (4.34) with $t=-k^{2}$ along with the Goldberger-Treiman relation

$$
g_{\pi N}=\frac{g_{A} m}{F_{\pi}} .
$$

Obviously for $\alpha_{16}^{a x, \mathrm{LO}} \neq-1$ the two results would disagree. The renormalizability condition, however, dictates the choice $\alpha_{16}^{a x, \mathrm{LO}}=-1$, which leads to the agreement with the on-shell result.

Based on the above results, we conjecture that the static two-loop contributions to the axial current operator can be obtained by taking the on-shell result for the corresponding form factors and dropping the energy-conserving deltafunction. This allows us to give the last missing piece in the current operator at order $Q$ without explicitly evaluating it using the method of UT:

$$
\begin{aligned}
& A_{1 \mathrm{~N}: \text { static }}^{0, a(Q)}=0 \\
& \vec{A}_{1 \mathrm{~N}: \text { static }}^{a(Q)}=-\frac{1}{2} \tau_{i}^{a} \vec{\sigma}_{i}\left(-f_{0}^{A} M_{\pi}^{2} k^{2}+f_{1}^{A} k^{4}+G_{A}^{\left(Q^{4}\right)}\left(-k^{2}\right)\right)+\frac{1}{8} \vec{k} \vec{k} \cdot \vec{\sigma}_{i} \tau_{i}^{a}\left(-4 f_{0}^{A} M_{\pi}^{2}-f_{1}^{P} k^{2}+G_{P}^{\left(Q^{2}\right)}\left(-k^{2}\right)\right) .
\end{aligned}
$$

To summarize, our final result for the single-nucleon axial charge and current operators up to order $Q$ can be expressed in terms of the nucleon form factors the following compact form

$$
\begin{aligned}
& A_{1 \mathrm{~N}}^{0, a}=-\frac{G_{A}\left(-k^{2}\right)}{2 m} \tau_{i}^{a} \vec{k}_{i} \cdot \vec{\sigma}_{i}+\frac{G_{P}\left(-k^{2}\right)}{8 m^{2}} \tau_{i}^{a} k_{0} \vec{k} \cdot \vec{\sigma}_{i}, \\
& \vec{A}_{1 \mathrm{~N}}^{a}=-\frac{G_{A}\left(-k^{2}\right)}{2} \tau_{i}^{a} \vec{\sigma}_{i}+\frac{G_{P}\left(-k^{2}\right)}{8 m^{2}} \tau_{i}^{a} \vec{k} \vec{k} \cdot \vec{\sigma}_{i}+\vec{A}_{1 \mathrm{~N}: 1 / m, \mathrm{UT}^{\prime}}^{a(Q)}+\vec{A}_{1 \mathrm{~N}: 1 / \mathrm{m}^{2}}^{a(Q)},
\end{aligned}
$$

where the last two terms are specified in Eqs. (4.13) and (4.18).

\section{TWO-NUCLEON AXIAL CHARGE AND CURRENT OPERATORS}

\section{A. Contributions at orders $Q^{-1}$ and $Q^{0}$}

As already mentioned above, the chiral expansion for the $2 \mathrm{~N}$ axial four-current operator starts at order $Q^{-1}$. The relevant diagrams generating the dominant contributions are shown in the first line of Fig. 10. As should be clear from the previous sections, all diagrams shown here and in the following are to be understood as representing the irreducible (i.e. non-iterative) pieces of the corresponding amplitudes. For the charge operator, the last two diagrams in the first line of Fig. 10 depend on the lowest-order unitary transformation

$$
(4),(5) \sim 1+\alpha_{16}^{a x, \mathrm{LO}} \text {. }
$$

For the current operator, the contributions of the leading-order tree-level graphs depend on unitary phases as follows:

$$
\begin{aligned}
& (1) \sim k_{0} \alpha_{1}^{a x}, \\
& (3) \sim k_{0}\left(\alpha_{25}^{a x}+\alpha_{26}^{a x}\right), \\
& (4) \sim \frac{k_{0} \alpha_{12}^{a x}}{k^{2}+M_{\pi}^{2}}[\ldots]+\frac{k_{0} \alpha_{13}^{a x}}{\left(k^{2}+M_{\pi}^{2}\right)^{2}}[\ldots], \\
& (5) \sim k_{0}\left(\alpha_{21}^{a x}+\alpha_{22}^{a x}+\alpha_{23}^{a x}\right) .
\end{aligned}
$$

As already pointed out, we adopt the choice of unitary phases $\alpha_{1}^{a x}=\alpha_{12}^{a x}=0, \alpha_{13}^{a x}=\alpha_{25}^{a x}+\alpha_{26}^{a x}=\alpha_{21}^{a x}+\alpha_{22}^{a x}+\alpha_{23}^{a x}=1$ and $\alpha_{16}^{a x, \mathrm{LO}}=-1$ which is dictated by renormalizability and by matching to the nuclear force. With this choice, the expressions for the one-pion-exchange contributions take the form

$$
\begin{aligned}
A_{2 \mathrm{~N}: 1 \pi}^{0, a}\left(Q^{-1}\right) & =-\frac{i g_{A} \vec{q}_{1} \cdot \vec{\sigma}_{1}\left[\boldsymbol{\tau}_{1} \times \boldsymbol{\tau}_{2}\right]^{a}}{4 F_{\pi}^{2}\left(q_{1}^{2}+M_{\pi}^{2}\right)}+1 \leftrightarrow 2, \\
\vec{A}_{2 \mathrm{~N}: 1 \pi}^{a\left(Q^{-1}\right)} & =0
\end{aligned}
$$




\section{leading order $\left(Q^{-1}\right)$ :}

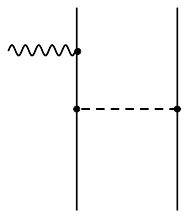

(1)

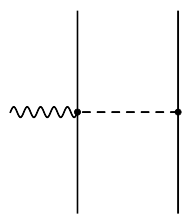

(2)

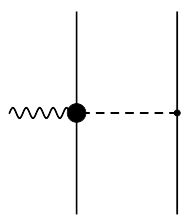

(6)

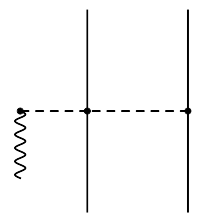

(3)

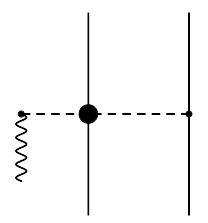

(7)

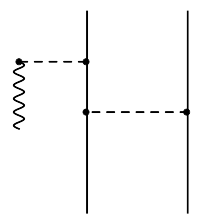

(4)

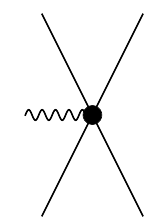

(8)

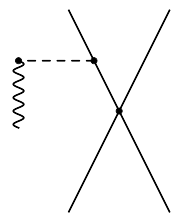

(5)

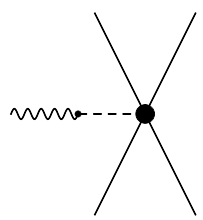

(9)

FIG. 10: Diagrams leading to the lowest-order contributions to the $2 \mathrm{~N}$ axial charge $\boldsymbol{A}_{2 \mathrm{~N}}^{0\left(Q^{-1}\right)}$ (upper line) and current operator $\overrightarrow{\boldsymbol{A}}_{2 \mathrm{~N}}^{\left(Q^{0}\right)}$ (lower line). Filled circles denote the subleading vertices from the effective Lagrangians $\mathcal{L}_{\pi N}^{(2)}$ and $\mathcal{L}_{\pi N N}^{(1)}$. Diagrams resulting from the interchange of the nucleon lines and/or application of the time reversal operation are not shown. For remaining notation see Fig. 1.

where $\vec{q}_{i}=\vec{p}_{i}^{\prime}-\vec{p}_{i}\left(\boldsymbol{\tau}_{i}\right)$ denotes the momentum transfer (Pauli isospin matrix) of nucleon $i$ and $q_{i} \equiv\left|\vec{q}_{i}\right|$. For the standard choice of the unitary phases, the short-range contribution of the last diagram in the first line of Fig. 10 vanishes:

$$
\begin{aligned}
A_{2 \mathrm{~N}: \text { cont }}^{0, a}\left(Q^{-1}\right) & =0, \\
\vec{A}_{2 \mathrm{~N}: \text { cont }}^{a\left(Q^{-1}\right)} & =0 .
\end{aligned}
$$

Notice that the choice $\alpha_{16}^{a x, \mathrm{LO}}=\alpha_{16}^{a x \text {,Static }}=\alpha_{16}^{a x, \text { Tadpole }}=-1$ switches off all pion-pole contributions to the charge. This choice is dictated by (off-shell) renormalizability of the single-nucleon charge operator, where terms proportional to $k_{0}$ are required to be finite.

Next, at order $Q^{0}$, one encounters the contributions to the $2 \mathrm{~N}$ axial current operator only, which originate from diagrams shown in the second line of Fig. 10. There are no charge contributions at this order. Again, the corresponding expressions are well-known and have the form

$$
\begin{aligned}
\vec{A}_{2 \mathrm{~N}: 1 \pi}^{a\left(Q^{0}\right)} & =\frac{g_{A}}{2 F_{\pi}^{2}} \frac{\vec{\sigma}_{1} \cdot \vec{q}_{1}}{q_{1}^{2}+M_{\pi}^{2}}\left\{\tau_{1}^{a}\left[-4 c_{1} M_{\pi}^{2} \frac{\vec{k}}{k^{2}+M_{\pi}^{2}}+2 c_{3}\left(\vec{q}_{1}-\frac{\vec{k} \vec{k} \cdot \vec{q}_{1}}{k^{2}+M_{\pi}^{2}}\right)\right]+c_{4}\left[\boldsymbol{\tau}_{1} \times \boldsymbol{\tau}_{2}\right]^{a}\left(\vec{q}_{1} \times \vec{\sigma}_{2}-\frac{\vec{k} \vec{k} \cdot \vec{q}_{1} \times \vec{\sigma}_{2}}{k^{2}+M_{\pi}^{2}}\right)\right. \\
& \left.-\frac{\kappa_{v}}{4 m}\left[\boldsymbol{\tau}_{1} \times \boldsymbol{\tau}_{2}\right]^{a} \vec{k} \times \vec{\sigma}_{2}\right\}+1 \leftrightarrow 2 \\
\vec{A}_{2 \mathrm{~N}: \text { cont }}^{a\left(Q^{0}\right)} & =-\frac{1}{4} D \tau_{1}^{a}\left(\vec{\sigma}_{1}-\frac{\vec{k} \vec{\sigma}_{1} \cdot \vec{k}}{k^{2}+M_{\pi}^{2}}\right)+1 \leftrightarrow 2
\end{aligned}
$$

where $c_{i}$ and $D$ denote the LECs from $\mathcal{L}_{\pi N}^{(2)}$ and $\mathcal{L}_{\pi N N}^{(1)}$, respectively, while $\kappa_{v}$ is the isovector anomalous magnetic moment of the nucleon. Further, we use the notation with $k \equiv|\vec{k}|$. It is easy to verify that the pion-pole contributions to the axial current fulfill the matching relations

$$
-\left.\frac{g_{A}}{2 F_{\pi}^{2}} \sum_{a} \tau_{3}^{a} \vec{\sigma}_{3} \cdot \vec{A}_{2 \mathrm{~N}: 1 \pi}^{a\left(Q^{0}\right)}\right|_{\vec{k}=-\vec{q}_{3}}=\left[V_{\mathrm{TPE}}^{3 \mathrm{NF}}\right]_{13}, \quad-\left.\frac{g_{A}}{2 F_{\pi}^{2}} \sum_{a} \tau_{3}^{a} \vec{\sigma}_{3} \cdot \vec{A}_{2 \mathrm{~N}: \operatorname{cont}}^{a\left(Q^{0}\right)}\right|_{\vec{k}=-\vec{q}_{3}}=\left[V_{\mathrm{OPE}}^{3 \mathrm{NF}}\right]_{12},
$$

where $\left[V_{\mathrm{TPE}}^{3 \mathrm{NF}}\right]_{13}\left(\left[V_{\mathrm{OPE}}^{3 \mathrm{NF}}\right]_{12}\right)$ denotes the part of the order- $Q^{3}$ two-pion exchange $3 \mathrm{~N}$ force in Eq. (2) (Eq. (10)) of $[9]$ symmetric with respect to the interchange of nucleons 1 and 3 (1 and 2). 


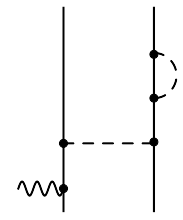

(1)<smiles>CCC(C)C(C)(C)C(C)C</smiles>

(8)<smiles>C[14CH][14C](C)(C)[14C](C)(C)[14CH3]</smiles>

(15)

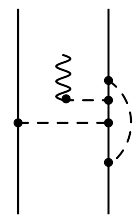

(22)

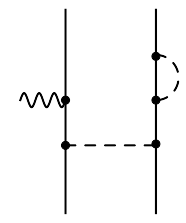

(2)

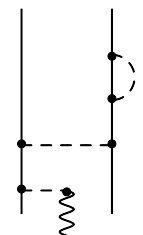

(9)<smiles>CC=[Te]C(C)(C)C(C)C</smiles>

(16)

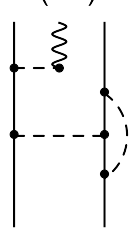

(23)<smiles>CCC(C)C(C)C(C)C</smiles>

(3)<smiles>C[14CH2][14C](C)(C)[14C](C)C</smiles>

(4)<smiles>CC(C)C(C)C(C)C</smiles>

(5)

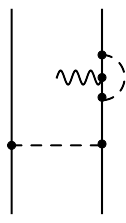

(6)

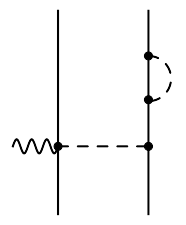

(7)

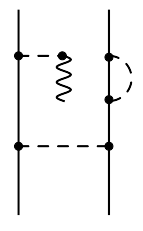

(10)<smiles>CCC(C)C(C)C(C)C</smiles>

(11)

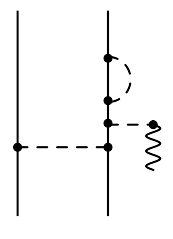

(12)

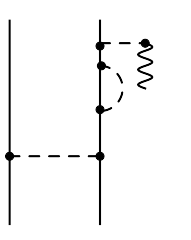

(13)

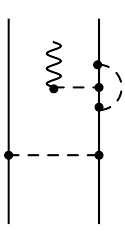

(14)<smiles>C[14CH]=[14C](C)[14CH](C)C</smiles>

(17)<smiles>CC=[V]C(C)(C)C</smiles>

(18)<smiles>CC1=C(C)C(C)(N)C1C</smiles>

(19)

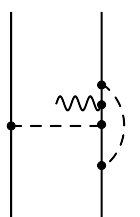

(20)

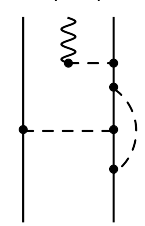

(21)

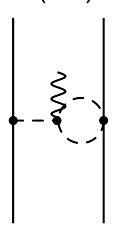

(28)

FIG. 11: Non-tadpole one-loop one-pion-exchange diagrams contributing to $\boldsymbol{A}_{2 \mathrm{~N}}^{\mu(Q)}$. For notation see Fig. 10.

\section{B. One-pion-exchange contributions at order $Q$}

In Fig. 11 we show all one-loop diagrams of non-tadpole type with a single pion being exchanged between the nucleons, which produce non-vanishing contributions to the axial charge and/or current operators. Specifically, we found that diagrams (1), (2), (7), (8), (11-18), (21), (23), (24), (26) and (28) generate non-vanishing contributions to the axial charge, from which those of the diagrams (1), (2), (11-14), (21), (23) do explicitly depend on the unitary phases in the following way:

$$
\begin{aligned}
(1),(2),(11),(21),(23) & \sim 1+\alpha_{16}^{a x, \mathrm{LO}}, \\
(12),(14) & \sim 1-\alpha_{4}^{a x}+\alpha_{5}^{a x}, \\
(13) & \sim 2+\alpha_{16}^{a x, \mathrm{LO}}-\alpha_{4}^{a x}+\alpha_{5}^{a x} .
\end{aligned}
$$

For the axial current, the diagrams (1-6), (9-15), (19-22) and (25-27) give non-vanishing contributions, from which those of graphs (1-5), (9-11), (13), (15) turn out to depend on the unitary phases:

$$
\begin{aligned}
(1),(2),(5) & \sim 1+\alpha_{1}^{a x}, \\
(3),(4) & \sim \alpha_{1}^{a x}, \\
(15) & \sim 2-\alpha_{25}^{a x}-\alpha_{26}^{a x}, \\
(9),(10),(13) & \sim\left(1-2 \alpha_{4}^{a x}+\alpha_{5}^{a x}-\alpha_{10}^{a x}\right) \frac{1}{\left(k^{2}+M_{\pi}^{2}\right)^{2}}[\ldots]+\left(1-\alpha_{12}^{a x}\right) \frac{1}{k^{2}+M_{\pi}^{2}}[\ldots],
\end{aligned}
$$




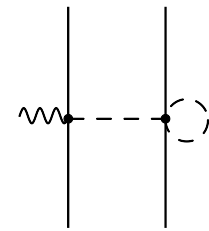

(1)

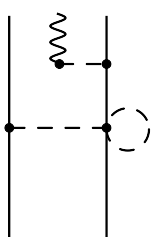

(7)

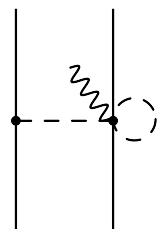

(2)

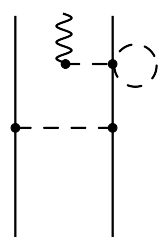

(8)

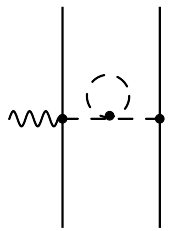

(3)

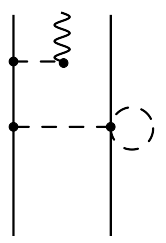

(9)

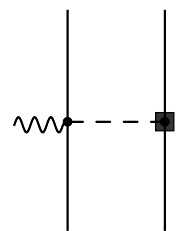

(4)

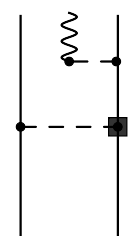

(10)

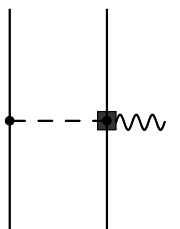

(5)

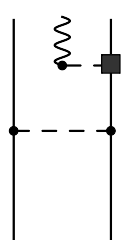

(11)

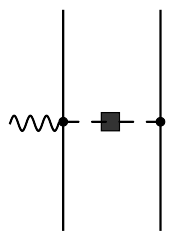

(6)

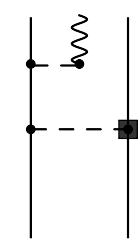

(12)

FIG. 12: One-pion-exchange tadpole and tree-level dragrams involving $d_{i}$-vertices from $\mathcal{L}_{\pi N}^{(3)}$ (denoted by filled squares) which contribute to $\boldsymbol{A}_{2 \mathrm{~N}}^{0(Q)}$. For remaining notation see Fig. 10 .

$$
(11),(12) \sim\left(1-2 \alpha_{4}^{a x}+\alpha_{5}^{a x}-\alpha_{10}^{a x}\right) \frac{1}{\left(k^{2}+M_{\pi}^{2}\right)^{2}}[\ldots]+\alpha_{12}^{a x} \frac{1}{k^{2}+M_{\pi}^{2}}[\ldots] .
$$

One observes that the contributions to the axial current operator from diagrams (3) and (4) vanish for the standard choice of the unitary phases. For the charge operator, diagrams (1), (2), (11), (21), (23) also turn out to yield vanishing contributions for the standard choice of the unitary phases.

Next, in Fig. 12 we show all non-vanishing one-pion-exchange tadpole diagrams and tree graphs involving $d_{i}$-vertices from $\mathcal{L}_{\pi N}^{(3)}$ and $l_{i}$-vertices from $\mathcal{L}_{\pi}^{(4)}$. We found that these diagrams contribute only to the axial charge operator. Further, graphs (7-12) yield contributions which depend on the unitary phases as

$$
\begin{aligned}
(7),(9),(10),(12) & \sim 1+\alpha_{16}^{a x, \text { LO }}, \\
(8) & \sim 1+\alpha_{16}^{a x, \text { Tadpole }}, \\
(11) & \sim 1+\alpha_{16}^{a x, \text { Static }},
\end{aligned}
$$

and vanish for the standard choice of the phases. Evaluating the contributions from the diagrams depicted in Figs. 11 and 12 for our standard choice of the unitary phases, replacing all bare LECs $l_{i}$ and $d_{i}$ in terms of their renormalized values as defined in Eq. (2.118), and expressing the results in terms of physical parameters $F_{\pi}, M_{\pi}$ and $g_{A}$, see e.g. [33], leads to our final result for the static order- $Q$ contributions to the $2 \mathrm{~N}$ one-pion-exchange axial current and charge operators:

$$
\begin{aligned}
\vec{A}_{2 \mathrm{~N}: 1 \pi}^{a(Q)} & =\frac{4 F_{\pi}^{2}}{g_{A}} \frac{\vec{q}_{1} \cdot \vec{\sigma}_{1}}{q_{1}^{2}+M_{\pi}^{2}}\left\{\left[\boldsymbol{\tau}_{1} \times \boldsymbol{\tau}_{2}\right]^{a}\left(\left[\vec{q}_{1} \times \vec{\sigma}_{2}\right] h_{1}\left(q_{2}\right)+\left[\vec{q}_{2} \times \vec{\sigma}_{2}\right] h_{2}\left(q_{2}\right)\right)+\boldsymbol{\tau}_{1}^{a}\left(\vec{q}_{1}-\vec{q}_{2}\right) h_{3}\left(q_{2}\right)\right\} \\
& +\frac{4 F_{\pi}^{2}}{g_{A}} \frac{\vec{q}_{1} \cdot \vec{\sigma}_{1} \vec{k}}{\left(k^{2}+M_{\pi}^{2}\right)\left(q_{1}^{2}+M_{\pi}^{2}\right)}\left\{\boldsymbol{\tau}_{1}^{a} h_{4}\left(q_{2}\right)+\left[\boldsymbol{\tau}_{1} \times \boldsymbol{\tau}_{2}\right]^{a} \vec{q}_{1} \cdot\left[\vec{q}_{2} \times \vec{\sigma}_{2}\right] h_{5}\left(q_{2}\right)\right\}+1 \leftrightarrow 2, \\
A_{2 \mathrm{~N}: 1 \pi}^{0, a(Q)} & =i \frac{4 F_{\pi}^{2}}{g_{A}} \frac{\vec{q}_{1} \cdot \vec{\sigma}_{1}}{q_{1}^{2}+M_{\pi}^{2}}\left\{\left[\boldsymbol{\tau}_{1} \times \boldsymbol{\tau}_{2}\right]^{a}\left(h_{6}\left(q_{2}\right)+k^{2} h_{7}\left(q_{2}\right)\right)+\tau_{1}^{a} \vec{q}_{1} \cdot\left[\vec{q}_{2} \times \vec{\sigma}_{2}\right] h_{8}\left(q_{2}\right)\right\}+1 \leftrightarrow 2,
\end{aligned}
$$

where the scalar functions $h_{i}\left(q_{2}\right)$ are given by

$$
\begin{aligned}
& h_{1}\left(q_{2}\right)=-\frac{g_{A}^{6} M_{\pi}}{128 \pi F_{\pi}^{6}}, \\
& h_{2}\left(q_{2}\right)=\frac{g_{A}^{4} M_{\pi}}{256 \pi F_{\pi}^{6}}+\frac{g_{A}^{4} A\left(q_{2}\right)\left(4 M_{\pi}^{2}+q_{2}^{2}\right)}{256 \pi F_{\pi}^{6}},
\end{aligned}
$$




$$
\begin{aligned}
h_{3}\left(q_{2}\right) & =\frac{g_{A}^{4}\left(g_{A}^{2}+1\right) M_{\pi}}{128 \pi F_{\pi}^{6}}+\frac{g_{A}^{4} A\left(q_{2}\right)\left(2 M_{\pi}^{2}+q_{2}^{2}\right)}{128 \pi F_{\pi}^{6}}, \\
h_{4}\left(q_{2}\right) & =\frac{g_{A}^{4}}{256 \pi F_{\pi}^{6}}\left(A\left(q_{2}\right)\left(2 M_{\pi}^{4}+5 M_{\pi}^{2} q_{2}^{2}+2 q_{2}^{4}\right)+\left(4 g_{A}^{2}+1\right) M_{\pi}^{3}+2\left(g_{A}^{2}+1\right) M_{\pi} q_{2}^{2}\right), \\
h_{5}\left(q_{2}\right) & =-\frac{g_{A}^{4}}{256 \pi F_{\pi}^{6}}\left(A\left(q_{2}\right)\left(4 M_{\pi}^{2}+q_{2}^{2}\right)+\left(2 g_{A}^{2}+1\right) M_{\pi}\right), \\
h_{6}\left(q_{2}\right) & =\frac{g_{A}^{2}\left(3\left(64+128 g_{A}^{2}\right) M_{\pi}^{2}+8\left(19 g_{A}^{2}+5\right) q_{2}^{2}\right)}{36864 \pi^{2} F_{\pi}^{6}}-\frac{g_{A}^{2}}{768 \pi^{2} F_{\pi}^{6}} L\left(q_{2}\right)\left(\left(8 g_{A}^{2}+4\right) M_{\pi}^{2}+\left(5 g_{A}^{2}+1\right) q_{2}^{2}\right) \\
& +\frac{\bar{d}_{18} g_{A} M_{\pi}^{2}}{8 F_{\pi}^{4}}-\frac{g_{A}^{2}\left(2 \bar{d}_{2}+\bar{d}_{6}\right)\left(M_{\pi}^{2}+q_{2}^{2}\right)}{16 F_{\pi}^{4}}-\frac{\bar{d}_{5} g_{A}^{2} M_{\pi}^{2}}{2 F_{\pi}^{4}}, \\
h_{7}\left(q_{2}\right) & =\frac{g_{A}^{2}\left(2 \bar{d}_{2}-\bar{d}_{6}\right)}{16 F_{\pi}^{4}}, \\
h_{8}\left(q_{2}\right) & =-\frac{g_{A}^{2}\left(\bar{d}_{15}-2 \bar{d}_{23}\right)}{8 F_{\pi}^{4}} .
\end{aligned}
$$

Here, the loop functions $L(q)$ and $A(q)$ are defined as

$$
L(q)=\frac{\sqrt{q^{2}+4 M_{\pi}^{2}}}{q} \ln \left(\frac{\sqrt{q^{2}+4 M_{\pi}^{2}}+q}{2 M_{\pi}}\right) \quad \text { and } \quad A(q)=\frac{1}{2 q} \arctan \left(\frac{q}{2 M_{\pi}}\right) .
$$

Notice that as desired, the pion-pole contributions to the current operator are directly related to the two-pion exchange contributions to the order- $Q^{4}\left(\mathrm{~N}^{3} \mathrm{LO}\right) 3 \mathrm{~N}$ force. In particular, the following relation holds true

$$
h_{4}\left(q_{2}\right)=\mathcal{A}^{(4)}\left(q_{2}\right), \quad h_{5}\left(q_{2}\right)=\mathcal{B}^{(4)}\left(q_{2}\right),
$$

where the scalar functions $\mathcal{A}^{(4)}\left(q_{2}\right)$ and $\mathcal{B}^{(4)}\left(q_{2}\right)$ entering the $3 \mathrm{~N}$ force are defined in Eq. (3.4) of [14].

Finally, apart from the static contributions, we need to take into account for the leading relativistic corrections emerging from tree-level diagrams with a single insertion of $1 / m$-vertices from the Lagrangian $\mathcal{L}_{\pi N}^{(2)}$. We stress again that due to the employed counting for the nucleon mass with $m \sim \Lambda_{b}^{2} / M_{\pi}$, these contributions are shifted one order higher relative to the ones emerging from tree-level diagrams with a single insertion of the $c_{i}$-vertices from $\mathcal{L}_{\pi N}^{(2)}$ shown in the second line of Fig. 10. In Fig. 13, we show all diagrams leading to non-vanishing contributions to the axial current operator. Notice that no $1 / m$-corrections to the $2 \mathrm{~N}$ axial charge operator appear at this order. Diagrams (1-17) turn out to induce a dependence on the unitary phases in the following way:

$$
\begin{aligned}
(1),(2) & \sim 1+2 \bar{\beta}_{8}+2 \alpha_{1}^{a x}, \\
(3) & \sim-1+2 \bar{\beta}_{8}+2 \alpha_{1}^{a x}, \\
(4) & \sim \alpha_{1}^{a x}, \\
(5) & \sim-1+2 \bar{\beta}_{8}, \\
(6),(14) & \sim-1+2 \bar{\beta}_{9}, \\
(7),(13) & \sim 1+2 \bar{\beta}_{9}, \\
(8),(9),(10) & \sim\left(-1+2 \alpha_{4}^{a x}-\alpha_{5}^{a x}+\alpha_{10}^{a x}\right) \frac{1}{\left(k^{2}+M_{\pi}^{2}\right)^{2}}[\ldots]+\left(-1-2 \bar{\beta}_{8}+2 \alpha_{12}^{a x}\right) \frac{1}{k^{2}+M_{\pi}^{2}}[\ldots], \\
(11) & \sim\left(-2+2 \alpha_{4}^{a x}-\alpha_{5}^{a x}+\alpha_{10}^{a x}+\alpha_{17}^{a x}+\alpha_{18}^{a x}+\alpha_{19}^{a x}\right) \frac{1}{\left(k^{2}+M_{\pi}^{2}\right)^{2}}[\ldots]+\alpha_{12}^{a x} \frac{1}{k^{2}+M_{\pi}^{2}}[\ldots], \\
(12) & \sim\left(-1+\alpha_{17}^{a x}+\alpha_{18}^{a x}+\alpha_{19}^{a x}\right) \frac{1}{\left(k^{2}+M_{\pi}^{2}\right)^{2}}[\ldots]+\left(-1+2 \bar{\beta}_{8}\right) \frac{1}{k^{2}+M_{\pi}^{2}}[\ldots], \\
(15) & \sim 1+\alpha_{16}^{a x, 1 / m}, \\
(16) & \sim-1+\alpha_{25}^{a x}+\alpha_{26}^{a x}, \\
(17) & \sim-2+\alpha_{25}^{a x}+\alpha_{26}^{a x} .
\end{aligned}
$$

Again, our standard choice of the unitary phases leads to some simplifications. In particular, it eliminates the contributions from diagrams (4) and (16). The explicit results for the $1 / m$-corrections to the one-pion-exchange 


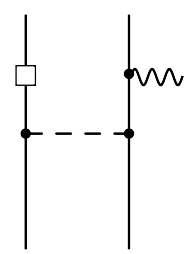

(1)

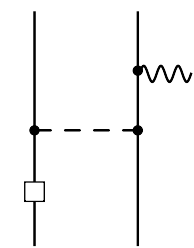

(2)

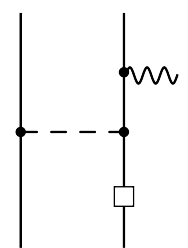

(3)

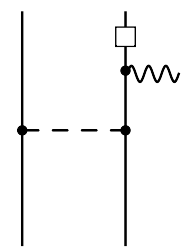

(4)

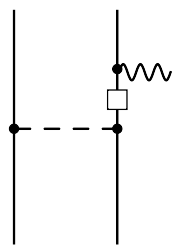

(5)

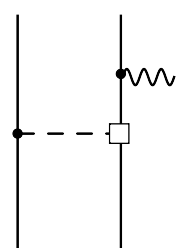

(6)<smiles>C[Te]C(C)C(C)C</smiles>

(7)

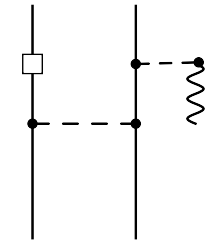

(8)

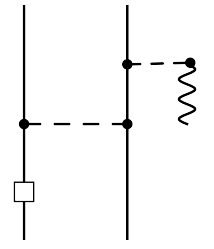

(9)

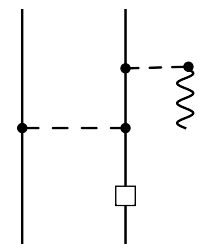

(10)

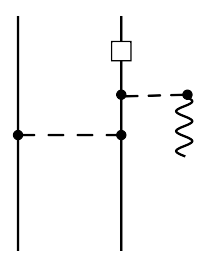

(11)

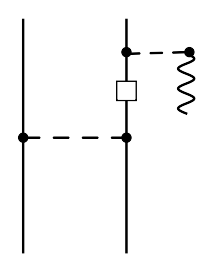

(12)

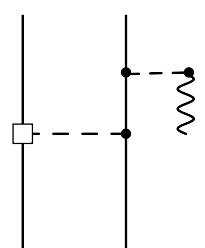

(13)

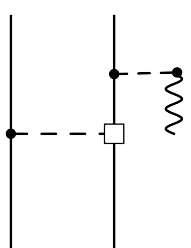

(14)

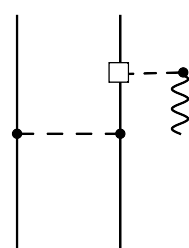

(15)

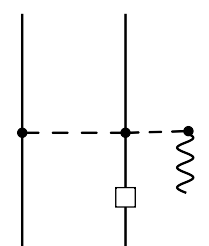

(16)

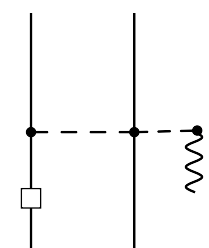

(17)

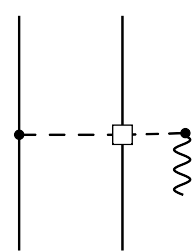

(18)

FIG. 13: One-pion exchange diagrams leading to non-vanishing $1 / m$-contributions to $\overrightarrow{\boldsymbol{A}}_{2 \mathrm{~N}}^{(Q)}$. Open rectangles refer to $1 / m$ vertices from $\mathcal{L}_{\pi N}^{(2)}$. For remaining notation see Fig. 10 .

current operator have the form

$$
\begin{aligned}
\vec{A}_{2 \mathrm{~N}: 1 \pi, 1 / m}^{a(Q)} & =\frac{g_{A}}{16 F_{\pi}^{2} m}\left\{i\left[\boldsymbol{\tau}_{1} \times \boldsymbol{\tau}_{2}\right]^{a}\left[\frac{1}{\left(q_{1}^{2}+M_{\pi}^{2}\right)^{2}}\left(\vec{B}_{1}-\frac{\vec{k} \vec{k} \cdot \vec{B}_{1}}{k^{2}+M_{\pi}^{2}}\right)+\frac{1}{q_{1}^{2}+M_{\pi}^{2}}\left(\frac{\vec{B}_{2}}{\left(k^{2}+M_{\pi}^{2}\right)^{2}}+\frac{\vec{B}_{3}}{k^{2}+M_{\pi}^{2}}+\vec{B}_{4}\right)\right]\right. \\
& \left.+\tau_{1}^{a}\left[\frac{1}{\left(q_{1}^{2}+M_{\pi}^{2}\right)^{2}}\left(\vec{B}_{5}-\frac{\vec{k} \vec{k} \cdot \vec{B}_{5}}{k^{2}+M_{\pi}^{2}}\right)+\frac{1}{q_{1}^{2}+M_{\pi}^{2}}\left(\frac{\vec{B}_{6}}{\left(k^{2}+M_{\pi}^{2}\right)^{2}}+\frac{\vec{B}_{7}}{k^{2}+M_{\pi}^{2}}+\vec{B}_{8}\right)\right]\right\}+1 \leftrightarrow 2, \quad(5.19)
\end{aligned}
$$

where the vector-valued quantities $\vec{B}_{i}$ depend on various momenta and the Pauli spin matrices and are given by

$$
\begin{aligned}
\vec{B}_{1} & =g_{A}^{2} \vec{q}_{1} \cdot \vec{\sigma}_{1}\left[-2\left(1+2 \bar{\beta}_{8}\right) \vec{q}_{1} \vec{k}_{1} \cdot \vec{q}_{1}-\left(1-2 \bar{\beta}_{8}\right)\left(2 \vec{q}_{1} \vec{k}_{2} \cdot \vec{q}_{1}-i \vec{q}_{1} \times \vec{\sigma}_{2} \vec{k} \cdot \vec{q}_{1}\right],\right. \\
\vec{B}_{2} & =\left(1-2 \bar{\beta}_{8}\right) g_{A}^{2} \vec{k} \vec{k} \cdot \vec{q}_{1} \vec{q}_{1} \cdot \vec{\sigma}_{1}\left[2 \vec{k} \cdot \vec{k}_{2}-i \vec{k} \cdot \vec{q}_{1} \times \vec{\sigma}_{2}\right], \\
\vec{B}_{3} & =2 \vec{k}\left[-g_{A}^{2}\left(\left(1+2 \bar{\beta}_{9}\right) \vec{k} \cdot \vec{q}_{1} \vec{k}_{1} \cdot \vec{\sigma}_{1}+\left(1-2 \bar{\beta}_{9}\right) \vec{q}_{1} \cdot \vec{\sigma}_{1}\left(\vec{k} \cdot \vec{k}_{2}+\vec{k}_{2} \cdot \vec{q}_{1}\right)\right)\right. \\
& \left.+\vec{q}_{1} \cdot \vec{\sigma}_{1}\left(\vec{k} \cdot \vec{k}_{2}+i \vec{k} \cdot \vec{q}_{1} \times \vec{\sigma}_{2}-\vec{k}_{1} \cdot \vec{q}_{1}+\vec{k}_{2} \cdot \vec{q}_{1}\right)\right], \\
\vec{B}_{4} & =g_{A}^{2}\left[2\left(1+2 \bar{\beta}_{9}\right) \vec{q}_{1} \vec{k}_{1} \cdot \vec{\sigma}_{1}+\left(1-2 \bar{\beta}_{9}\right) \vec{q}_{1} \cdot \vec{\sigma}_{1}\left(2 \vec{k}_{2}-i \vec{k} \times \vec{\sigma}_{2}\right)\right]-2 \vec{q}_{1} \cdot \vec{\sigma}_{1}\left(i \vec{q}_{1} \times \vec{\sigma}_{2}-i \vec{k} \times \vec{\sigma}_{2}+2 \vec{k}_{2}\right), \\
\vec{B}_{5} & =g_{A}^{2} \vec{q}_{1} \cdot \vec{\sigma}_{1}\left[\left(1-2 \bar{\beta}_{8}\right)\left(\vec{q}_{1} \vec{k} \cdot \vec{q}_{1}-2 i \vec{q}_{1} \times \vec{\sigma}_{2} \vec{k}_{2} \cdot \vec{q}_{1}\right)-2 i\left(1+2 \bar{\beta}_{8}\right) \vec{q}_{1} \times \vec{\sigma}_{2} \vec{k}_{1} \cdot \vec{q}_{1}\right], \\
\vec{B}_{6} & =-\left(1-2 \bar{\beta}_{8}\right) g_{A}^{2} \vec{k} \vec{q}_{1} \cdot \vec{\sigma}_{1}\left[\left(\vec{k} \cdot \vec{q}_{1}\right)^{2}-2 i \vec{k} \cdot \vec{k}_{2} \vec{k} \cdot \vec{q}_{1} \times \vec{\sigma}_{2}\right], \\
\vec{B}_{7} & =g_{A}^{2} \vec{k}\left[\left(1-2 \bar{\beta}_{9}\right) \vec{q}_{1} \cdot \vec{\sigma}_{1}\left(-2 i\left(\vec{k} \cdot \vec{k}_{2} \times \vec{\sigma}_{2}+\vec{k}_{2} \cdot \vec{q}_{1} \times \vec{\sigma}_{2}\right)+k^{2}+q_{1}^{2}\right)-2 i\left(1+2 \bar{\beta}_{9}\right) \vec{k}_{1} \cdot \vec{\sigma}_{1} \vec{k} \cdot \vec{q}_{1} \times \vec{\sigma}_{2}\right], \\
\vec{B}_{8} & =-g_{A}^{2}\left[\left(1-2 \bar{\beta}_{9}\right) \vec{q}_{1} \cdot \vec{\sigma}_{1}\left(\vec{k}-2 i \vec{k}_{2} \times \vec{\sigma}_{2}\right)-2 i\left(1+2 \bar{\beta}_{9}\right) \vec{q}_{1} \times \vec{\sigma}_{2} \vec{k}_{1} \cdot \vec{\sigma}_{1}\right] .
\end{aligned}
$$

It is not quite straightforward to make a connection between the derived relativistic corrections to the axial current operator and the corresponding $1 / m$-terms appearing in the $3 \mathrm{~N}$ force at $\mathrm{N}^{3} \mathrm{LO}$. This is because the later ones also 


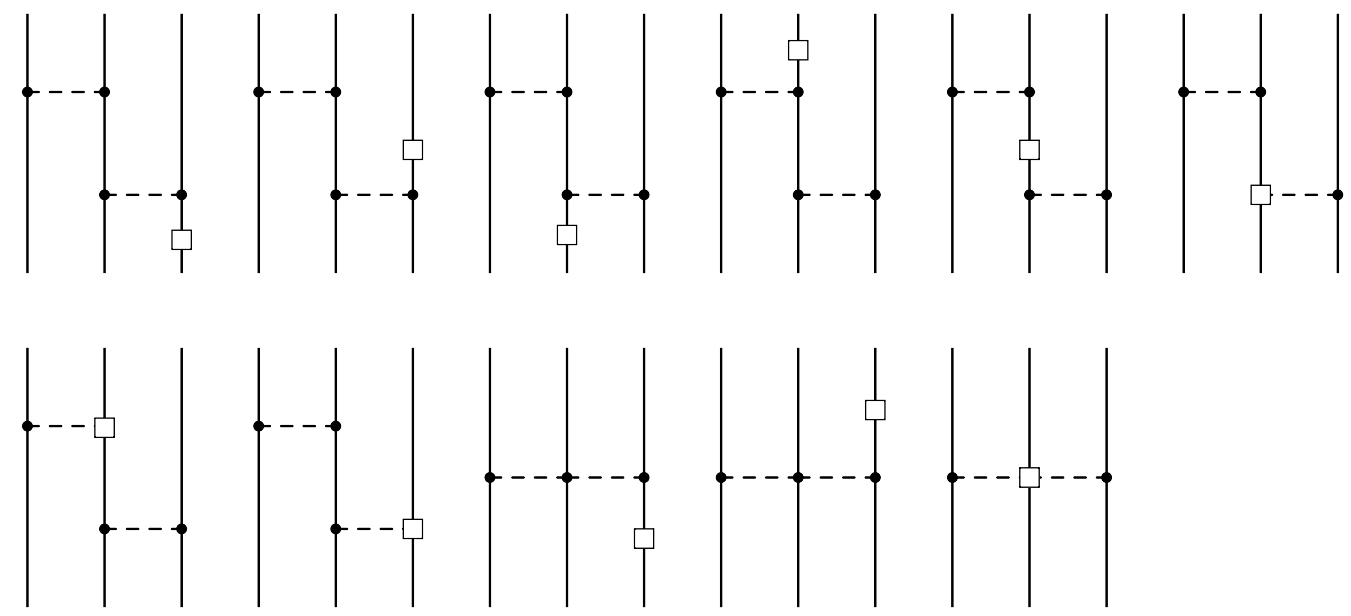

FIG. 14: Diagrams leading to non-vanishing relativistic corrections to the two-pion exchange $3 \mathrm{~N}$ force at $\mathrm{N}^{3} \mathrm{LO}$ which do not include the $1 / m$-vertices from $\mathcal{L}_{\pi N}^{(2)}$ (shown by open squares) at the leftmost nucleon line. Time reversed diagrams are not shown. For remaining notation see Fig. 1.

receive contributions from diagrams involving an insertion of the $1 / m$-vertex at the nucleon line, which we regard as being attributed to the axial-vector source (i.e. the leftmost nucleon line in the $3 \mathrm{~N}$ force shown in Fig. 1), and which is connected with the two-nucleon system via one-pion exchange. Thus, to establish the connection, we have to consider only those topologies in the $3 \mathrm{~N}$ force, which do not include such contributions. In Fig. 14 we show all relevant diagrams which generate non-vanishing terms in the $3 \mathrm{~N}$ force. We have calculated the resulting contributions $\left[V_{\mathrm{TPE}, 1 / m}^{3 \mathrm{NF}}\right]_{\text {modified }}$ and verified the validity of the relation

$$
\left[V_{\mathrm{TPE}, 1 / m}^{3 \mathrm{NF}}\right]_{\text {modified }}=-\left.\frac{g_{A}}{2 F_{\pi}^{2}} \sum_{a} \tau_{3}^{a} \vec{\sigma}_{3} \cdot \vec{A}_{2 \mathrm{~N}: 1 \pi, 1 / m}^{a(Q)}\right|_{\vec{k}=-\vec{q}_{3}, k^{2}=-M_{\pi}^{2}}+\mathcal{O}\left(\left(k^{2}+M_{\pi}^{2}\right)^{0}\right) .
$$

Last but not least, there are also contributions proportional to the energy transfer $k_{0}$ stemming from time-derivatives of the unitary transformations in diagrams shown in Fig. 10. As already mentioned earlier, $k_{0}$ counts as a quantity of order $Q^{3}$ so that the contributions from diagrams (1),(3) and (4) of Fig. 10 are shifted from order $Q^{-1}$ to order $Q$. For the standard choice of unitary phases we obtain

$$
\begin{aligned}
& A_{2 \mathrm{~N}: 1 \pi, \mathrm{UT}^{\prime}}^{0, a(Q)}=0, \\
& \vec{A}_{2 \mathrm{~N}: 1 \pi, \mathrm{UT}^{\prime}}^{a(Q)}=-i \frac{g_{A}}{8 F_{\pi}^{2}} \frac{k_{0} \vec{k} \vec{q}_{1} \cdot \vec{\sigma}_{1}}{\left(k^{2}+M_{\pi}^{2}\right)\left(q_{1}^{2}+M_{\pi}^{2}\right)}\left(\left[\boldsymbol{\tau}_{1} \times \boldsymbol{\tau}_{2}\right]^{a}\left(1-\frac{2 g_{A}^{2} \vec{k} \cdot \vec{q}_{1}}{k^{2}+M_{\pi}^{2}}\right)-\frac{2 g_{A}^{2} \tau_{1}^{a} \vec{k} \cdot\left[\vec{q}_{1} \times \vec{\sigma}_{2}\right]}{k^{2}+M_{\pi}^{2}}\right)+1 \leftrightarrow 2 .
\end{aligned}
$$

The current contribution in Eq. (5.23) involves the pion production operator, which, again, can be matched to the corresponding expressions in the $3 \mathrm{~N}$ force. The energy transfer $k_{0}$ can then be written as the difference of the initial and final kinetic energies of the third nucleon,

$$
k_{0}=\frac{p_{3}^{2}}{2 m}-\frac{p_{3}^{\prime 2}}{2 m}
$$

Notice that $k_{0}$ refers to the outgoing (incoming) energy transfer of the third nucleon (subsystem of the nucleons 1 and 2). Thus, we need to consider a subset of diagrams generating relativistic corrections to the two-pion exchange $3 \mathrm{~N}$ force with the kinetic-energy insertions at the third nucleon, i.e. at the leftmost nucleon lines in Fig. 15. The explicit expressions for the corresponding $3 \mathrm{~N}$ force contributions are given by

$$
\left[V_{\mathrm{TPE}, k_{0}}^{3 \mathrm{NF}}\right]_{\text {modified }}=-i \frac{g_{A}^{2}}{16 F_{\pi}^{4}} \frac{k_{0} \vec{q}_{3} \cdot \vec{\sigma}_{3} \vec{q}_{1} \cdot \vec{\sigma}_{1}}{\left(q_{3}^{2}+M_{\pi}^{2}\right)\left(q_{1}^{2}+M_{\pi}^{2}\right)}\left[\boldsymbol{\tau}_{3} \cdot\left[\boldsymbol{\tau}_{1} \times \boldsymbol{\tau}_{2}\right]\left(1+\left(1+2 \bar{\beta}_{8}\right) \frac{g_{A}^{2} \vec{q}_{3} \cdot \vec{q}_{1}}{q_{3}^{2}+M_{\pi}^{2}}\right)\right.
$$




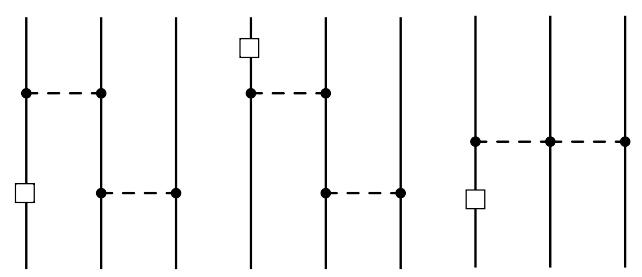

FIG. 15: Diagrams leading to non-vanishing contributions to the two-pion exchange $3 \mathrm{~N}$ force at $\mathrm{N}^{3} \mathrm{LO}$ which include the $1 / m$-vertices from $\mathcal{L}_{\pi N}^{(2)}$ (shown by open squares) at the leftmost nucleon line. The indicated kinetic energy insertions at the leftmost nucleon line can be identically expressed in terms of the energy transfer $k_{0}$ of Eq. (5.24). Time reversed diagrams are not shown.

$$
\left.+\left(1+2 \bar{\beta}_{8}\right) \frac{g_{A}^{2} \boldsymbol{\tau}_{1} \cdot \boldsymbol{\tau}_{3} \vec{q}_{3} \cdot\left[\vec{q}_{1} \times \vec{\sigma}_{2}\right]}{q_{3}^{2}+M_{\pi}^{2}}\right]+1 \leftrightarrow 2,
$$

where $k_{0}$ is specified in Eq. (5.24). We then find that the difference between the $3 \mathrm{~N}$ force in the above equation and the contribution reconstructed from the axial current in Eq. (5.23) is given by

$$
\begin{aligned}
& {\left[V_{\mathrm{TPE}, k_{0}}^{3 \mathrm{NF}}\right]_{\text {modified }}+\left.\frac{g_{A}}{2 F_{\pi}^{2}} \sum_{a} \tau_{3}^{a} \vec{\sigma}_{3} \cdot \vec{A}_{2 \mathrm{~N}: 1 \pi, \mathrm{UT}^{\prime}}^{a}\right|_{\vec{k}=-\vec{q}_{3}, k^{2}=-M_{\pi}^{2}}=} \\
& \left(1-2 \bar{\beta}_{8}\right) i \frac{g_{A}^{4}}{16 F_{\pi}^{4}} \frac{k_{0} \vec{q}_{3} \cdot \vec{\sigma}_{3} \vec{q}_{1} \cdot \vec{\sigma}_{1}}{\left(q_{3}^{2}+M_{\pi}^{2}\right)^{2}\left(q_{1}^{2}+M_{\pi}^{2}\right)}\left(\boldsymbol{\tau}_{3} \cdot\left[\boldsymbol{\tau}_{1} \times \boldsymbol{\tau}_{2}\right] \vec{q}_{3} \cdot \vec{q}_{1}+\boldsymbol{\tau}_{1} \cdot \boldsymbol{\tau}_{3} \vec{q}_{3} \cdot\left[\vec{q}_{1} \times \vec{\sigma}_{2}\right]\right)+1 \leftrightarrow 2 .
\end{aligned}
$$

This shows that the matching of $\vec{A}_{2 \mathrm{~N}: 1 \pi, \mathrm{UT}^{\prime}}^{a(Q)}$ to the $3 \mathrm{~N}$ force is only possible for $\bar{\beta}_{8}=1 / 2$.

\section{Two-pion-exchange contributions}

We now turn to the two-pion exchange contributions. In Fig. 16, we show all diagrams yielding non-vanishing results for the axial charge and/or current operator with two exchanged pions. For the axial charge, diagrams (1), (4), (5), (7), (10), (13), (17), (19), (21) give non-vanishing contributions, from which those of graphs (4), (10), (17), (19), (21) appear to depend on the unitary phases via

$$
(4),(10),(17),(19),(21) \sim 1+\alpha_{16}^{a x, L O} .
$$

Clearly, all these contributions vanish for our standard choice. For the axial current operator, we find non-vanishing results from diagrams (2-6), (8-12), (14-18), (20), which in the case of graphs (2-6) depend on the unitary phases according to

$$
\begin{aligned}
(2),(3) & \sim 1+2 \alpha_{1}^{a x} \\
(5) & \sim-3+2 \alpha_{25}^{a x}+2 \alpha_{26}^{a x}, \\
(4),(6) & \sim\left(-1+2 \alpha_{4}^{a x}-\alpha_{5}^{a x}+\alpha_{10}^{a x}\right) \frac{1}{\left(k^{2}+M_{\pi}^{2}\right)^{2}}[\cdots]+\left(-1+2 \alpha_{12}^{a x}\right) \frac{1}{k^{2}+M_{\pi}^{2}} .
\end{aligned}
$$

Notice that the contributions involving second-order pion-pole terms resulting from diagrams (4), (6) vanish for our standard choice of the unitary phases. The final results for the two-pion exchange operators read

$$
\begin{aligned}
\vec{A}_{2 \mathrm{~N}: 2 \pi}^{a(Q)} & =\frac{2 F_{\pi}^{2}}{g_{A}} \frac{\vec{k}}{k^{2}+M_{\pi}^{2}}\left\{\tau_{1}^{a}\left(-\vec{q}_{1} \cdot \vec{\sigma}_{2} \vec{q}_{1} \cdot \vec{k} g_{1}\left(q_{1}\right)+\vec{q}_{1} \cdot \vec{\sigma}_{2} g_{2}\left(q_{1}\right)-\vec{k} \cdot \vec{\sigma}_{2} g_{3}\left(q_{1}\right)\right)+\tau_{2}^{a}\left(-\vec{q}_{1} \cdot \vec{\sigma}_{1} \vec{q}_{1} \cdot \vec{k} g_{4}\left(q_{1}\right)\right.\right. \\
& \left.-\vec{k} \cdot \vec{\sigma}_{1} g_{5}\left(q_{1}\right)-\vec{q}_{1} \cdot \vec{\sigma}_{2} \vec{q}_{1} \cdot \vec{k} g_{6}\left(q_{1}\right)+\vec{q}_{1} \cdot \vec{\sigma}_{2} g_{7}\left(q_{1}\right)+\vec{k} \cdot \vec{\sigma}_{2} \vec{q}_{1} \cdot \vec{k} g_{8}\left(q_{1}\right)-\vec{k} \cdot \vec{\sigma}_{2} g_{9}\left(q_{1}\right)\right) \\
& \left.+\left[\boldsymbol{\tau}_{1} \times \boldsymbol{\tau}_{2}\right]^{a}\left(-\vec{q}_{1} \cdot\left[\vec{\sigma}_{1} \times \vec{\sigma}_{2}\right] \vec{q}_{1} \cdot \vec{k} g_{10}\left(q_{1}\right)+\vec{q}_{1} \cdot\left[\vec{\sigma}_{1} \times \vec{\sigma}_{2}\right] g_{11}\left(q_{1}\right)-\vec{q}_{1} \cdot \vec{\sigma}_{2} \vec{q}_{1} \cdot\left[\vec{q}_{2} \times \vec{\sigma}_{1}\right] g_{12}\left(q_{1}\right)\right)\right\}
\end{aligned}
$$




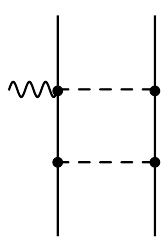

(1)<smiles>CC1C(C)C12C(C)[C@H]2C</smiles>

(7)<smiles>CC1(C)C2(C)C(C)(C)C12C</smiles>

(13)

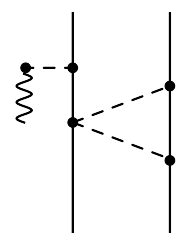

(19)

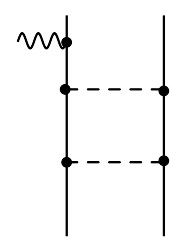

(2)

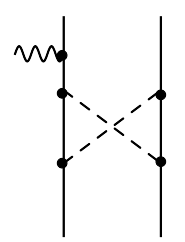

(8)

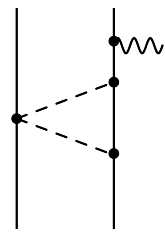

(14)

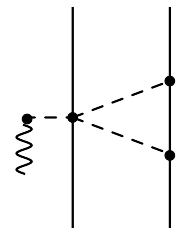

(20)

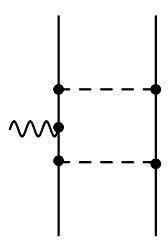

(3)

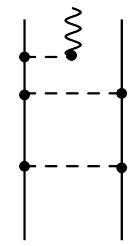

(4)

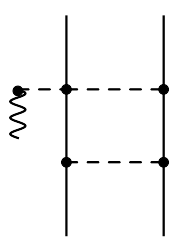

(5)

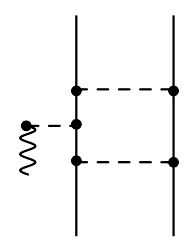

(6)

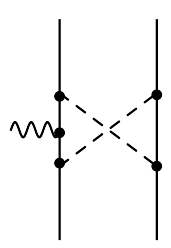

(9)

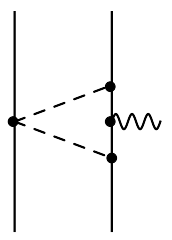

(15)

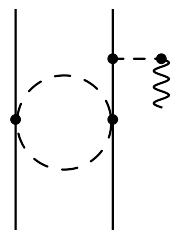

(21)

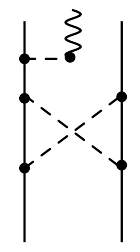

(10)

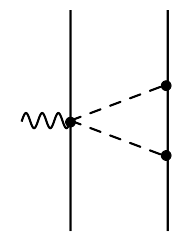

(16)<smiles>CC1C(C)C12C(C)C2(C)C</smiles>

(11)

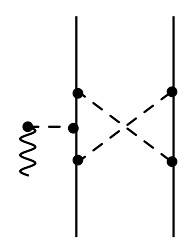

(12)

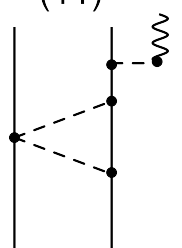

(17)

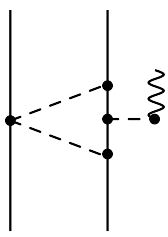

(18)

FIG. 16: Two-pion-exchange diagrams contributing to $\boldsymbol{A}_{2 \mathrm{~N}}^{\mu(Q)}$. For notation see Fig. 10.

$$
\begin{aligned}
& +\frac{2 F_{\pi}^{2}}{g_{A}}\left\{\vec{q}_{1}\left(\tau_{2}^{a} \vec{q}_{1} \cdot \vec{\sigma}_{1} g_{13}\left(q_{1}\right)+\tau_{1}^{a} \vec{q}_{1} \cdot \vec{\sigma}_{2} g_{14}\left(q_{1}\right)\right)-\tau_{1}^{a} \vec{\sigma}_{2} g_{15}\left(q_{1}\right)-\tau_{2}^{a} \vec{\sigma}_{2} g_{16}\left(q_{1}\right)-\tau_{2}^{a} \vec{\sigma}_{1} g_{17}\left(q_{1}\right)\right\} \\
& +1 \leftrightarrow 2
\end{aligned}
$$

$$
A_{2 \mathrm{~N}: 2 \pi}^{0, a(Q)}=i \frac{2 F_{\pi}^{2}}{g_{A}}\left\{\left[\boldsymbol{\tau}_{1} \times \boldsymbol{\tau}_{2}\right]^{a} \vec{q}_{1} \cdot \vec{\sigma}_{2} g_{18}\left(q_{1}\right)+\tau_{2}^{a} \vec{q}_{1} \cdot\left[\vec{\sigma}_{1} \times \vec{\sigma}_{2}\right] g_{19}\left(q_{1}\right)\right\}+1 \leftrightarrow 2,
$$

where the scalar functions $g_{i}\left(q_{1}\right)$ are defined as

$$
\begin{aligned}
& g_{1}\left(q_{1}\right)=\frac{g_{A}^{4} A\left(q_{1}\right)\left(\left(8 g_{A}^{2}-4\right) M_{\pi}^{2}+\left(g_{A}^{2}+1\right) q_{1}^{2}\right)}{256 \pi F_{\pi}^{6} q_{1}^{2}}-\frac{g_{A}^{4} M_{\pi}\left(\left(8 g_{A}^{2}-4\right) M_{\pi}^{2}+\left(3 g_{A}^{2}-1\right) q_{1}^{2}\right)}{256 \pi F_{\pi}^{6} q_{1}^{2}\left(4 M_{\pi}^{2}+q_{1}^{2}\right)} \\
& g_{2}\left(q_{1}\right)=\frac{g_{A}^{4} A\left(q_{1}\right)\left(2 M_{\pi}^{2}+q_{1}^{2}\right)}{128 \pi F_{\pi}^{6}}+\frac{g_{A}^{4} M_{\pi}}{128 \pi F_{\pi}^{6}}, \\
& g_{3}\left(q_{1}\right)=-\frac{g_{A}^{4} A\left(q_{1}\right)\left(\left(8 g_{A}^{2}-4\right) M_{\pi}^{2}+\left(3 g_{A}^{2}-1\right) q_{1}^{2}\right)}{256 \pi F_{\pi}^{6}}-\frac{\left(3 g_{A}^{2}-1\right) g_{A}^{4} M_{\pi}}{256 \pi F_{\pi}^{6}}, \\
& g_{4}\left(q_{1}\right)=-\frac{g_{A}^{6} A\left(q_{1}\right)}{128 \pi F_{\pi}^{6}} \\
& g_{5}\left(q_{1}\right)=-q_{1}^{2} g_{4}\left(q_{1}\right),
\end{aligned}
$$




$$
\begin{aligned}
g_{6}\left(q_{1}\right) & =g_{8}\left(q_{1}\right)=g_{10}\left(q_{1}\right)=g_{12}\left(q_{1}\right)=0, \\
g_{7}\left(q_{1}\right) & =\frac{g_{A}^{4} A\left(q_{1}\right)\left(2 M_{\pi}^{2}+q_{1}^{2}\right)}{128 \pi F_{\pi}^{6}}+\frac{\left(2 g_{A}^{2}+1\right) g_{A}^{4} M_{\pi}}{128 \pi F_{\pi}^{6}}, \\
g_{9}\left(q_{1}\right) & =\frac{g_{A}^{6} M_{\pi}}{64 \pi F_{\pi}^{6}}, \\
g_{11}\left(q_{1}\right) & =-\frac{g_{A}^{4} A\left(q_{1}\right)\left(4 M_{\pi}^{2}+q_{1}^{2}\right)}{512 \pi F_{\pi}^{6}}-\frac{g_{A}^{4} M_{\pi}}{512 \pi F_{\pi}^{6}}, \\
g_{13}\left(q_{1}\right) & =-\frac{g_{A}^{6} A\left(q_{1}\right)}{128 \pi F_{\pi}^{6}}, \\
g_{14}\left(q_{1}\right) & =\frac{g_{A}^{4} A\left(q_{1}\right)\left(\left(8 g_{A}^{2}-4\right) M_{\pi}^{2}+\left(g_{A}^{2}+1\right) q_{1}^{2}\right)}{256 \pi F_{\pi}^{6} q_{1}^{2}}+\frac{g_{A}^{4} M_{\pi}\left(\left(4-8 g_{A}^{2}\right) M_{\pi}^{2}+\left(1-3 g_{A}^{2}\right) q_{1}^{2}\right)}{256 \pi F_{\pi}^{6} q_{1}^{2}\left(4 M_{\pi}^{2}+q_{1}^{2}\right)} \\
g_{15}\left(q_{1}\right) & =\frac{g_{A}^{4} A\left(q_{1}\right)\left(\left(8 g_{A}^{2}-4\right) M_{\pi}^{2}+\left(3 g_{A}^{2}-1\right) q_{1}^{2}\right)}{256 \pi F_{\pi}^{6}}+\frac{\left(3 g_{A}^{2}-1\right) g_{A}^{4} M_{\pi}}{256 \pi F_{\pi}^{6}}, \\
g_{16}\left(q_{1}\right) & =\frac{g_{A}^{4} A\left(q_{1}\right)\left(2 M_{\pi}^{2}+q_{1}^{2}\right)}{64 \pi F_{\pi}^{6}}+\frac{g_{A}^{4} M_{\pi}}{64 \pi F_{\pi}^{6}}, \\
g_{17}\left(q_{1}\right) & =-\frac{g_{A}^{6} q_{1}^{2} A\left(q_{1}\right)}{128 \pi F_{\pi}^{6}}, \\
g_{18}\left(q_{1}\right) & =\frac{g_{A}^{2} L\left(q_{1}\right)\left(\left(4-8 g_{A}^{2}\right) M_{\pi}^{2}+\left(1-3 g_{A}^{2}\right) q_{1}^{2}\right)}{128 \pi^{2} F_{\pi}^{6}\left(4 M_{\pi}^{2}+q_{1}^{2}\right)}, \\
g_{19}\left(q_{1}\right) & =\frac{g_{A}^{4} L\left(q_{1}\right)}{32 \pi^{2} F_{\pi}^{6}} .
\end{aligned}
$$

Our standard choice of the unitary phases ensures that the $2 \mathrm{~N}$ irreducible pion production amplitude entering the pion-pole contributions to the axial current operator equals the one appearing in the one-pion-two-pion-exchange $3 \mathrm{~N}$ force at $\mathrm{N}^{3} \mathrm{LO}$. This manifests itself in the relations

$$
g_{i}\left(q_{1}\right)=F_{i}\left(q_{1}\right), \quad i=1, \ldots, 12
$$

where $F_{i}\left(q_{1}\right)$ are the scalar functions entering the corresponding $3 \mathrm{~N}$ force and defined in Eq. (3.2) of [15]. ${ }^{8}$ Notice further that the loop contributions to the current operator are finite in dimensional regularization, whereas the divergences in the loop integrals appearing in the axial charge are absorbed into redefinition of LECs accompanying the contact operators to be specified below.

\section{Short-range contributions at order $Q$}

We first consider static contributions and begin with tree-level diagrams, which emerge from the terms in the effective Lagrangian $\mathcal{L}_{N N}^{(2)}$ involving one derivative and one insertion of the axial vector source. While there are no contributions to the current at this order, four independent structures appear in the charge operator. Using the notation of Ref. [56], the tree-level contributions read:

$$
\begin{aligned}
\vec{A}_{2 \mathrm{~N}: \text { cont }}^{a(Q)} & =0 \\
A_{2 \mathrm{~N}: \text { cont }}^{0, a(Q)} & =i z_{1}\left[\boldsymbol{\tau}_{1} \times \boldsymbol{\tau}_{2}\right]^{a} \vec{\sigma}_{1} \cdot \vec{q}_{2}+i z_{2}\left[\boldsymbol{\tau}_{1} \times \boldsymbol{\tau}_{2}\right]^{a} \vec{\sigma}_{1} \cdot \vec{q}_{1}+i z_{3} \tau_{1}^{a} \vec{q}_{2} \cdot \vec{\sigma}_{1} \times \vec{\sigma}_{2} \\
& +z_{4}\left(\tau_{1}^{a}-\tau_{2}^{a}\right)\left(\vec{\sigma}_{1}-\vec{\sigma}_{2}\right) \cdot \vec{k}_{1}+1 \leftrightarrow 2,
\end{aligned}
$$

with $z_{i}$ denoting the corresponding LECs.

\footnotetext{
${ }^{8}$ Notice that in [15], we have only shown explicitly non-polynomial contributions to the scalar functions $F_{i}$ since the polynomial terms, which for dimensional reasons have to be momentum-independent and proportional to $M_{\pi}$, only lead to finite shifts of the LEC $c_{D}$. Eq. (5.32) is valid both for polynomial and non-polynomial parts.
} 
Next, we show in Fig. 17 all non-vanishing one-loop diagrams involving a single insertion of the leading contact interactions from $\mathcal{L}_{N N}^{(0)}$. For the axial charge, diagrams (3), (5), (8), (10), (15-17), (20), (25-30) are found to give non-vanishing contributions, from which those of graphs (3), (5), (8), (10), (15-17), (20), (25-30) depend on the choice of the unitary phases:

$$
\begin{aligned}
(3),(5),(8),(10),(15-17),(20),(28-30) & \sim 1+\alpha_{16}^{a x, \mathrm{LO}}, \\
(25) & \sim 2-\alpha_{4}^{a x}+\alpha_{5}^{a x}+\alpha_{16}^{a x, \mathrm{LO}}, \\
(26),(27) & \sim-1+\alpha_{4}^{a x}-\alpha_{5}^{a x} .
\end{aligned}
$$

For our standard choice of the unitary phases, we obtain a vanishing result for the pion-pole terms in the axial charge. All ultraviolet divergences in the loop contributions to the axial charge operator are cancelled by the corresponding counterterms upon expressing the bare LECs $z_{i}$ in terms of their renormalized values $\bar{z}_{i}$ via

$$
z_{i}=\bar{z}_{i}+\beta_{z_{i}} \frac{1}{F_{\pi}^{4}}\left(\lambda+\frac{1}{16 \pi^{2}} \ln \left(\frac{M_{\pi}}{\mu}\right)\right) .
$$

The corresponding $\beta$-functions read:

$$
\begin{aligned}
& \beta_{z_{1}}=\frac{\left(3 g_{A}^{2}-1\right) g_{A}}{4}, \\
& \beta_{z_{2}}=-\frac{\left(5 g_{A}^{2}+1\right) g_{A}}{12}, \\
& \beta_{z_{3}}=-g_{A}^{3}, \\
& \beta_{z_{4}}=0 .
\end{aligned}
$$

The remaining finite contributions to the charge operator at order $Q$ can be absorbed into a redefinition of the renormalized LECs $\bar{z}_{i}$ via the following shifts:

$$
\begin{aligned}
& \bar{z}_{1} \rightarrow \bar{z}_{1}+\frac{\left(1-g_{A}^{2}\right) g_{A}}{128 \pi^{2} F_{\pi}^{4}} \\
& \bar{z}_{2} \rightarrow \bar{z}_{2}+\frac{g_{A}}{4 F_{\pi}^{2}}\left(2 \bar{d}_{2}+\bar{d}_{6}\right) \\
& \bar{z}_{3} \rightarrow \frac{g_{A}^{3}}{32 \pi^{2} F_{\pi}^{4}}
\end{aligned}
$$

For the current operator, diagrams (1-9), (11-14), (17-30) are found to yield non-vanishing contributions, from which those of graphs (1-5), (21), (23-25), (27-30) depend on the unitary phases:

$$
\begin{aligned}
(1),(2) & \sim 1+2 \alpha_{1}^{a x}, \\
(3) & \sim\left(-1+2 \alpha_{4}^{a x}-\alpha_{5}^{a x}+\alpha_{10}^{a x}\right) \frac{1}{\left(k^{2}+M_{\pi}^{2}\right)^{2}}[\ldots]+\left(-1+2 \alpha_{12}^{a x}\right) \frac{1}{k^{2}+M_{\pi}^{2}}[\ldots], \\
(4) & \sim\left(-2+2 \alpha_{4}^{a x}-\alpha_{5}^{a x}+\alpha_{10}^{a x}+\alpha_{21}^{a x}+\alpha_{22}^{a x}+\alpha_{23}^{a x}\right) \frac{1}{\left(k^{2}+M_{\pi}^{2}\right)^{2}}[\ldots]+\left(-1+2 \alpha_{12}^{a x}\right) \frac{1}{k^{2}+M_{\pi}^{2}}[\ldots], \\
(5),(24),(28-30) & \sim-1+\alpha_{21}^{a x}+\alpha_{22}^{a x}+\alpha_{23}^{a x}, \\
(21) & \sim 1+\alpha_{1}^{a x}, \\
(23) & \sim \alpha_{1}^{a x}, \\
(25) & \sim\left(-1+2 \alpha_{4}^{a x}-\alpha_{5}^{a x}+\alpha_{10}^{a x}\right) \frac{1}{\left(k^{2}+M_{\pi}^{2}\right)^{2}}[\ldots]+\left(-1+\alpha_{12}^{a x}\right) \frac{1}{k^{2}+M_{\pi}^{2}}[\ldots], \\
(27) & \sim\left(-2+2 \alpha_{4}^{a x}-\alpha_{5}^{a x}+\alpha_{10}^{a x}+\alpha_{21}^{a x}+\alpha_{22}^{a x}+\alpha_{23}^{a x}\right) \frac{1}{\left(k^{2}+M_{\pi}^{2}\right)^{2}}[\ldots]+\alpha_{12}^{a x} \frac{1}{k^{2}+M_{\pi}^{2}}[\ldots] .
\end{aligned}
$$

For our standard choice, we find a vanishing result for the short-range current operator after renormalization. This is consistent with the matching condition to the nuclear forces, since the static one-pion-contact 3NF's vanish after antisymmetrization. Thus, after renormalization and antisymmetrization, there are no static contributions to the short-range axial current operator at order $Q$, while those to the charge operator are given by Eq. (5.33). 


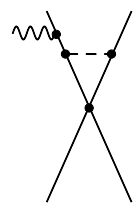

(1)

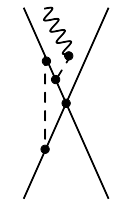

(9)

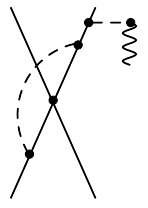

(17)

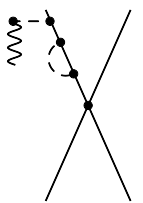

(25)

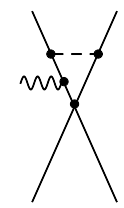

(2)

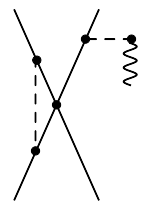

(10)

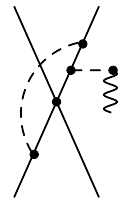

(18)

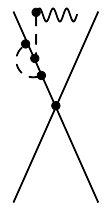

(26)

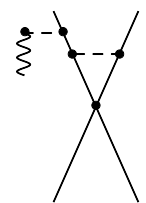

(3)

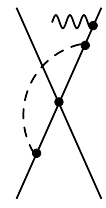

(11)

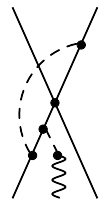

(19)

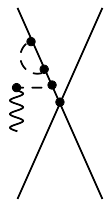

(27)

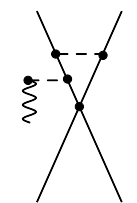

(4)

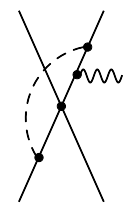

(12)

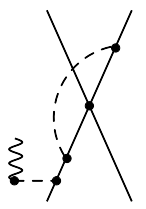

(20)

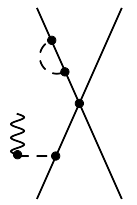

(28)

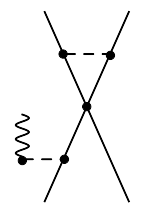

(5)

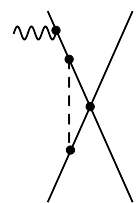

(6)

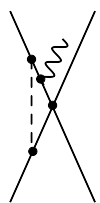

(7)

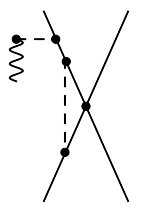

(8)

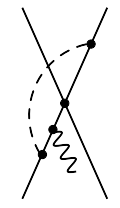

(13)

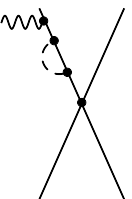

(21)

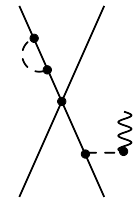

(29)

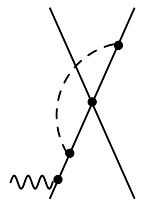

(14)

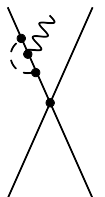

(22)

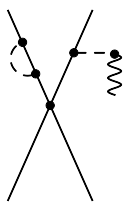

(30)

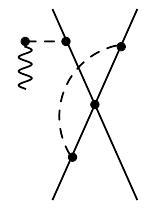

(15)

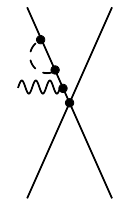

(23)

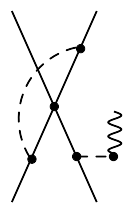

(16)

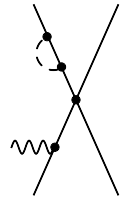

(24)

FIG. 17: Loop diagrams with contact interactions contributing to $\boldsymbol{A}_{2 \mathrm{~N}}^{\mu(Q)}$. Solid dots denote vertices from $\mathcal{L}_{\pi N}^{(1)}, \mathcal{L}_{\pi}^{(2)}$ or $\mathcal{L}_{N N}^{(0)}$. For remaining notation see Fig. 10.

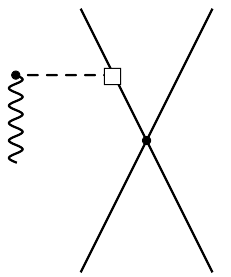

(1)

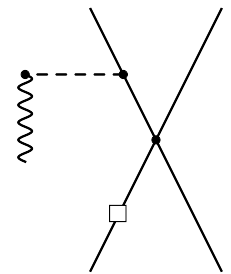

(2)

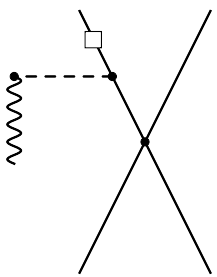

(3)

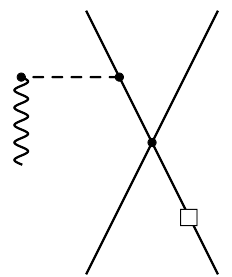

(4)

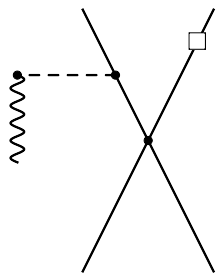

(5)

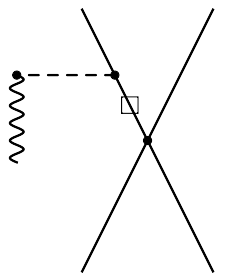

(6)

FIG. 18: Tree-level diagrams with contact interactions leading to $1 / m$-contributions to $\overrightarrow{\boldsymbol{A}}_{2 \mathrm{~N}}^{(Q)}$. Solid dots (open squares) denote vertices from $\mathcal{L}_{\pi N}^{(1)}\left(1 / m\right.$-corrections from $\left.\mathcal{L}_{\pi N}^{(2)}\right)$. For remaining notation see Fig. 10.

In addition to the static terms considered above, one also encounters relativistic $1 / m$ corrections involving a single insertion of the LO contact interactions from $\mathcal{L}_{N N}^{(0)}$. We find that the contributions to the axial charge disappear regardless of the choice of the unitary phases. For the vector current, non-vanishing $1 / m$-corrections emerge from the diagrams shown in Fig. 18. The unitary phase dependence of these diagrams is given by

$$
\begin{aligned}
(1) & \sim 1+\alpha_{16}^{a x, 1 / m}, \\
(2),(4),(5) & \sim \alpha_{21}^{a x}+\alpha_{22}^{a x}+\alpha_{23}^{a x}-1, \\
(3) & \sim \alpha_{17}^{a x}+\alpha_{18}^{a x}+\alpha_{19}^{a x}+\alpha_{21}^{a x}+\alpha_{22}^{a x}+\alpha_{23}^{a x}-2,
\end{aligned}
$$




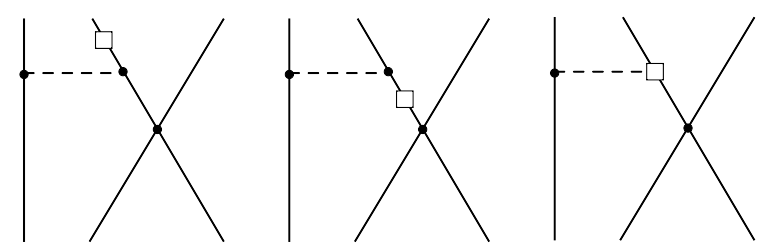

FIG. 19: Diagrams leading to non-vanishing relativistic corrections to the one-pion-exchange-contact $3 \mathrm{~N}$ force at $\mathrm{N}^{3} \mathrm{LO}$ which do not include the $1 / m$-vertices from $\mathcal{L}_{\pi N}^{(2)}$ (shown by open squares) at the leftmost nucleon line. Time reversed diagrams are not shown. For remaining notation see Fig. 1.

$$
\text { (6) } \sim \alpha_{17}^{a x}+\alpha_{18}^{a x}+\alpha_{19}^{a x}-1
$$

For our standard choice of the unitary phases, we find the following result for the axial current operator:

$$
\begin{aligned}
\vec{A}_{2 \mathrm{~N}: \text { cont }, 1 / m}^{a(Q)} & =-\frac{g_{A}}{4 m} \frac{\vec{k}}{k^{2}+M_{\pi}^{2}} \tau_{1}^{a}\left\{\left(1-2 \bar{\beta}_{9}\right)\left(C_{S} \vec{q}_{2} \cdot \vec{\sigma}_{1}+C_{T}\left(\vec{q}_{2} \cdot \vec{\sigma}_{2}+2 i \vec{k}_{1} \cdot \vec{\sigma}_{1} \times \vec{\sigma}_{2}\right)\right)\right. \\
& \left.-\frac{1-2 \bar{\beta}_{8}}{k^{2}+M_{\pi}^{2}}\left(C_{S} \vec{k} \cdot \overrightarrow{q_{2}} \vec{k} \cdot \vec{\sigma}_{1}+C_{T}\left(\vec{k} \cdot \overrightarrow{q_{2}} \vec{k} \cdot \vec{\sigma}_{2}+2 i \vec{k} \cdot \vec{k} \vec{k}_{1} \cdot \vec{\sigma}_{1} \times \vec{\sigma}_{2}\right)\right)\right\}+1 \leftrightarrow 2 .
\end{aligned}
$$

To verify the matching condition with the $3 \mathrm{~N}$ force, we have calculated the contribution $\left[V_{1 \pi-\text { cont, } 1 / m}^{3 \mathrm{NF}}\right]$ modified of the diagrams shown in Fig. 19, which do not involve $1 / m$-corrections at the leftmost nucleon line. We have then verified that the following relation is indeed valid:

$$
\left[V_{1 \pi-\text { cont }, 1 / m}^{3 \mathrm{NF}}\right]_{\text {modified }}=-\left.\frac{g_{A}}{2 F_{\pi}^{2}} \sum_{a} \tau_{3}^{a} \vec{\sigma}_{3} \cdot \vec{A}_{2 \mathrm{~N}: \text { cont }, 1 / m}^{a(Q)}\right|_{\vec{k}=-\vec{q}_{3}, k^{2}=-M_{\pi}^{2}}+\mathcal{O}\left(\left(k^{2}+M_{\pi}^{2}\right)^{0}\right) .
$$

Finally, there are also contributions from diagram (5) of Fig. 10, which are proportional to the energy transfer $k^{0}$. For the standard choice of unitary phases we obtain the following result:

$$
\begin{aligned}
& A_{2 \mathrm{~N}: \text { cont, } \mathrm{UT}^{\prime}}^{0, a(Q)}=0, \\
& \vec{A}_{2 \mathrm{~N}: \text { cont, } \mathrm{UT}^{\prime}}^{a(Q)}=-i k_{0} \vec{k} \frac{g_{A} C_{T} \vec{k} \cdot \vec{\sigma}_{1}\left[\boldsymbol{\tau}_{1} \times \boldsymbol{\tau}_{2}\right]^{a}}{\left(k^{2}+M_{\pi}^{2}\right)^{2}}+1 \leftrightarrow 2 .
\end{aligned}
$$

Similar to the one-pion exchange current, this expression matches the corresponding terms in the $3 \mathrm{~N}$ force for the choice of $\bar{\beta}_{8}=1 / 2$.

\section{THREE-NUCLEON AXIAL CURRENTS}

We now turn to the $3 \mathrm{~N}$ axial current operators, whose dominant contributions appear at order $Q$ from tree-level diagrams constructed solely from the lowest-order vertices. In Fig. 20, we show all graphs which yield non-vanishing contributions to the axial current, and that do not involve contact interactions. Interestingly, we find only vanishing contributions to the $3 \mathrm{~N}$ charge operator at this order. Out of the 26 diagrams shown in Fig. 20, graphs (1-18) yield expressions which depend on the unitary phases as follows:

$$
\begin{aligned}
(1-4) & \sim 1+\alpha_{1}^{a x}, \\
(5-7) & \sim \alpha_{1}^{a x} \\
(12),(13) & \sim-1+\alpha_{25}^{a x}+\alpha_{26}^{a x}, \\
(17),(18) & \sim-2+\alpha_{25}^{a x}+\alpha_{26}^{a x},
\end{aligned}
$$




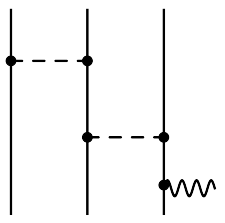

(1)

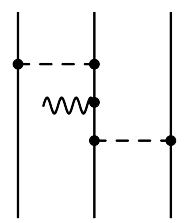

(7)

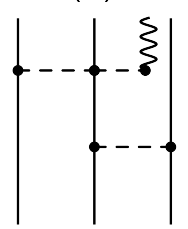

(13)

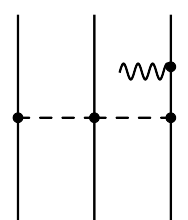

(19)

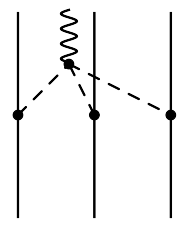

(25)

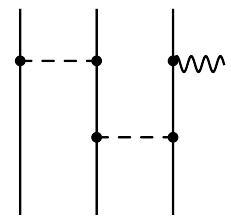

(2)

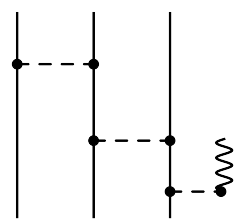

(8)

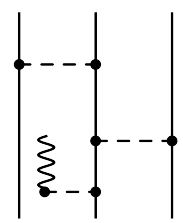

(14)

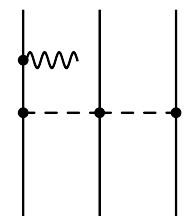

(20)

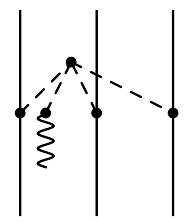

(26)

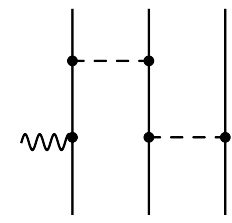

(3)

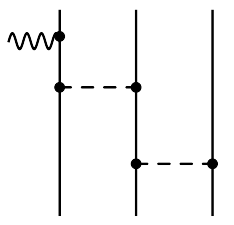

(4)

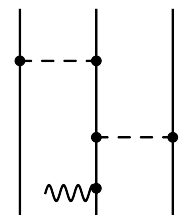

(5)

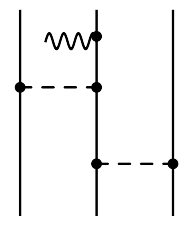

(6)

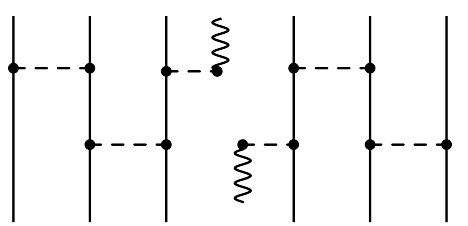

(9)

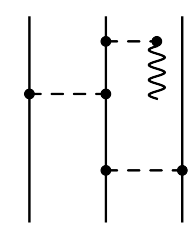

(15)

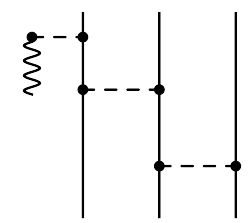

(11)

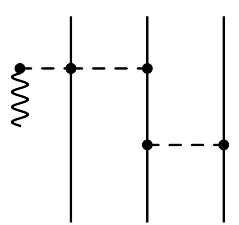

(17)

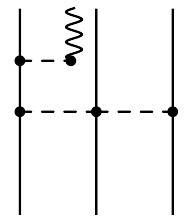

(23)

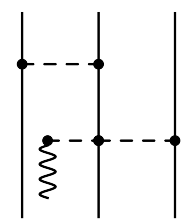

(12)

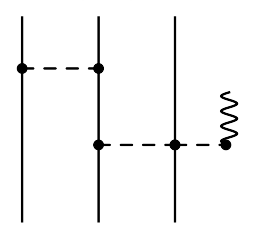

(18)

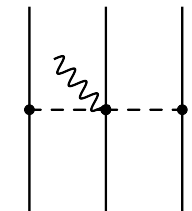

(21)

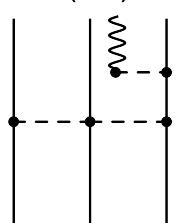

(22)

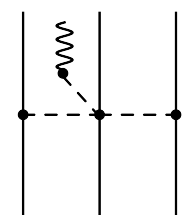

(24)

FIG. 20: Tree-level two-pion exchange diagrams leading to non-vanishing contributions to $\vec{A}_{3 \mathrm{~N}}^{(Q)}$. For notation see Fig. 10.

$$
\begin{aligned}
(8-11) & \sim\left(-1+2 \alpha_{4}^{a x}-\alpha_{5}^{a x}+\alpha_{10}^{a x}\right) \frac{1}{\left(k^{2}+M_{\pi}^{2}\right)^{2}}[\ldots]+\left(-1+\alpha_{12}^{a x}\right) \frac{1}{k^{2}+M_{\pi}^{2}}[\ldots], \\
(14-16) & \sim\left(-1+2 \alpha_{4}^{a x}-\alpha_{5}^{a x}+\alpha_{10}^{a x}\right) \frac{1}{\left(k^{2}+M_{\pi}^{2}\right)^{2}}[\ldots]+\alpha_{12}^{a x} \frac{1}{k^{2}+M_{\pi}^{2}}[\ldots] .
\end{aligned}
$$

For our standard choice of the unitary phases, the contributions from diagrams (5-7) and (12-16) as well as all expressions involving second-order pion-pole terms are found to vanish. In order to facilitate a comparison with the four-nucleon force at $\mathrm{N}^{3} \mathrm{LO}$, we write the resulting expression for the $3 \mathrm{~N}$ axial current $\vec{A}_{3 \mathrm{~N}: \pi}^{a(Q)}$ in the form

$$
\vec{A}_{3 \mathrm{~N}: \pi}^{a(Q)}=-\frac{2 F_{\pi}^{2}}{g_{A}} \sum_{i=1}^{8} \vec{C}_{i}^{a}+5 \text { permutations }
$$

where

$$
\begin{aligned}
\vec{C}_{1}^{a} & =\frac{g_{A}^{6}}{16 F_{\pi}^{6}} \vec{q}_{2} \cdot \vec{\sigma}_{2}\left[\tau _ { 2 } ^ { a } \left[\vec{q}_{3}\left(\left(\vec{q}_{2} \cdot \vec{q}_{3}+q_{2}^{2}\right)\left(\boldsymbol{\tau}_{1} \cdot \boldsymbol{\tau}_{3}-\vec{\sigma}_{1} \cdot \vec{\sigma}_{3}\right)+\vec{q}_{2} \cdot \vec{\sigma}_{1}\left(\vec{q}_{2} \cdot \vec{\sigma}_{3}+\vec{q}_{3} \cdot \vec{\sigma}_{3}\right)\right)\right.\right. \\
& -\vec{q}_{2}\left(\vec{q}_{3} \cdot \vec{\sigma}_{1}\left(\vec{q}_{2} \cdot \vec{\sigma}_{3}+\vec{q}_{3} \cdot \vec{\sigma}_{3}\right)-\left(\vec{q}_{2} \cdot \vec{q}_{3}+q_{3}^{2}\right) \vec{\sigma}_{1} \cdot \vec{\sigma}_{3}-\left(\vec{q}_{2} \cdot \vec{q}_{3}+q_{2}^{2}\right) \boldsymbol{\tau}_{1} \cdot \boldsymbol{\tau}_{3}\right)
\end{aligned}
$$




$$
\begin{aligned}
& \left.-\vec{\sigma}_{3}\left(\left(\vec{q}_{2} \cdot \vec{q}_{3}+q_{3}^{2}\right) \vec{q}_{2} \cdot \vec{\sigma}_{1}-\left(\vec{q}_{2} \cdot \vec{q}_{3}+q_{2}^{2}\right) \vec{q}_{3} \cdot \vec{\sigma}_{1}\right)\right]-\left[\boldsymbol{\tau}_{2} \times \boldsymbol{\tau}_{3}\right]^{a}\left(\vec{q}_{2} \times \vec{\sigma}_{1}+\vec{q}_{3} \times \vec{\sigma}_{1}\right)\left(\vec{q}_{2} \cdot \vec{q}_{3}+q_{2}^{2}\right) \\
& \left.-\left(\overrightarrow{q_{2}}+\vec{q}_{3}\right)\left(\left[\boldsymbol{\tau}_{1} \times \boldsymbol{\tau}_{2}\right]^{a} \vec{q}_{2} \cdot \vec{q}_{3} \times \vec{\sigma}_{3}+\tau_{3}^{a} \boldsymbol{\tau}_{1} \cdot \boldsymbol{\tau}_{2}\left(\vec{q}_{2} \cdot \overrightarrow{q_{3}}+q_{2}^{2}\right)\right)\right] \frac{1}{\left[q_{2}^{2}+M_{\pi}^{2}\right]\left[\left(\vec{q}_{1}-\vec{k}\right)^{2}+M_{\pi}^{2}\right]^{2}}, \\
& \vec{C}_{2}^{a}=\frac{g_{A}^{4}}{32 F_{\pi}^{6}} \frac{\vec{q}_{2} \cdot \vec{\sigma}_{2}\left[\left[\boldsymbol{\tau}_{2} \times \boldsymbol{\tau}_{3}\right]^{a}\left(\vec{k} \times \vec{\sigma}_{1}-\vec{q}_{1} \times \vec{\sigma}_{1}\right)+\left(\vec{k}-\vec{q}_{1}\right)\left(\tau_{3}^{a} \boldsymbol{\tau}_{1} \cdot \boldsymbol{\tau}_{2}-\tau_{2}^{a} \boldsymbol{\tau}_{1} \cdot \boldsymbol{\tau}_{3}\right)\right]}{\left[q_{2}^{2}+M_{\pi}^{2}\right]\left[\left(\vec{q}_{1}-\vec{k}\right)^{2}+M_{\pi}^{2}\right]}, \\
& \vec{C}_{3}^{a}=\frac{g_{A}^{4}}{32 F_{\pi}^{6}} \frac{\left(\vec{k}-2 \vec{q}_{3}\right) \tau_{3}^{a} \boldsymbol{\tau}_{1} \cdot \boldsymbol{\tau}_{2} \vec{q}_{1} \cdot \vec{\sigma}_{1} \vec{q}_{2} \cdot \vec{\sigma}_{2}\left(\vec{k} \cdot \vec{\sigma}_{3}-2 \vec{q}_{1} \cdot \vec{\sigma}_{3}\right)}{\left[q_{1}^{2}+M_{\pi}^{2}\right]\left[q_{2}^{2}+M_{\pi}^{2}\right]\left[q_{3}^{2}+M_{\pi}^{2}\right]}, \\
& \vec{C}_{4}^{a}=\frac{g_{A}^{4}}{32 F_{\pi}^{6}} \frac{\vec{\sigma}_{1} \vec{q}_{2} \cdot \vec{\sigma}_{2} \vec{q}_{3} \cdot \vec{\sigma}_{3}\left(\tau_{1}^{a} \boldsymbol{\tau}_{2} \cdot \boldsymbol{\tau}_{3}-\tau_{3}^{a} \boldsymbol{\tau}_{1} \cdot \boldsymbol{\tau}_{2}\right)}{\left[q_{2}^{2}+M_{\pi}^{2}\right]\left[q_{3}^{2}+M_{\pi}^{2}\right]} \\
& \vec{C}_{5}^{a}=\frac{g_{A}^{6}}{16 F_{\pi}^{6}} \vec{k} \vec{q}_{1} \cdot \vec{\sigma}_{1}\left[-\tau_{1}^{a} \vec{q}_{1} \cdot \vec{q}_{2} \times \vec{\sigma}_{2}\left(\vec{k} \cdot \vec{q}_{1} \times \vec{\sigma}_{3}+\vec{k} \cdot \vec{q}_{2} \times \vec{\sigma}_{3}\right)-\left[\boldsymbol{\tau}_{1} \times \boldsymbol{\tau}_{3}\right]^{a} \vec{q}_{1} \cdot \vec{q}_{2} \times \vec{\sigma}_{2}\left(\vec{k} \cdot \overrightarrow{q_{1}}+\vec{k} \cdot \vec{q}_{2}\right)\right. \\
& +\left[\boldsymbol{\tau}_{1} \times \boldsymbol{\tau}_{2}\right]^{a}\left(\vec{q}_{1} \cdot \vec{q}_{2}+q_{1}^{2}\right)\left(\vec{k} \cdot \vec{q}_{1} \times \vec{\sigma}_{3}+\vec{k} \cdot \vec{q}_{2} \times \vec{\sigma}_{3}\right)-\left(\boldsymbol{\tau}_{2} \cdot \boldsymbol{\tau}_{3} \tau_{1}^{a}-\boldsymbol{\tau}_{1} \cdot \boldsymbol{\tau}_{3} \tau_{2}^{a}\right) \\
& \left.\times\left(\vec{q}_{1} \cdot \vec{q}_{2}+q_{1}^{2}\right)\left(\vec{k} \cdot \vec{q}_{1}+\vec{k} \cdot \vec{q}_{2}\right)\right] \frac{1}{\left[k^{2}+M_{\pi}^{2}\right]\left[q_{1}^{2}+M_{\pi}^{2}\right]\left[\left(\vec{q}_{1}+\vec{q}_{2}\right)^{2}+M_{\pi}^{2}\right]^{2}}, \\
& \vec{C}_{6}^{a}=-\frac{g_{A}^{4}}{32 F_{\pi}^{6}} \vec{k} \vec{q}_{1} \cdot \vec{\sigma}_{1}\left[\left(\vec{k} \cdot \vec{q}_{1} \times \vec{\sigma}_{3}+\vec{k} \cdot \vec{q}_{2} \times \vec{\sigma}_{3}\right)\left[\boldsymbol{\tau}_{1} \times \boldsymbol{\tau}_{2}\right]^{a}-\left(\vec{k} \cdot \vec{q}_{1}+\vec{k} \cdot \vec{q}_{2}+\vec{q}_{1} \cdot \vec{q}_{2}+q_{1}^{2}\right)\right. \\
& \left.\times\left(\boldsymbol{\tau}_{2} \cdot \boldsymbol{\tau}_{3} \tau_{1}^{a}-\boldsymbol{\tau}_{1} \cdot \boldsymbol{\tau}_{3} \tau_{2}^{a}\right)-\vec{q}_{1} \cdot \vec{q}_{2} \times \vec{\sigma}_{2}\left[\boldsymbol{\tau}_{1} \times \boldsymbol{\tau}_{3}\right]^{a}\right] \frac{1}{\left[k^{2}+M_{\pi}^{2}\right]\left[q_{1}^{2}+M_{\pi}^{2}\right]\left[\left(\vec{q}_{1}+\vec{q}_{2}\right)^{2}+M_{\pi}^{2}\right]}, \\
& \vec{C}_{7}^{a}=-\frac{g_{A}^{4}}{32 F_{\pi}^{6}} \frac{\vec{k} \vec{q}_{1} \cdot \vec{\sigma}_{1} \vec{q}_{2} \cdot \vec{\sigma}_{2} \vec{q}_{3} \cdot \vec{\sigma}_{3} \boldsymbol{\tau}_{1} \cdot \boldsymbol{\tau}_{2} \tau_{3}^{a}\left(M_{\pi}^{2}+2 \vec{q}_{1} \cdot \vec{q}_{2}+q_{1}^{2}+q_{2}^{2}\right)}{\left[k^{2}+M_{\pi}^{2}\right]\left[q_{1}^{2}+M_{\pi}^{2}\right]\left[q_{2}^{2}+M_{\pi}^{2}\right]\left[q_{3}^{2}+M_{\pi}^{2}\right]}, \\
& \vec{C}_{8}^{a}=-\frac{g_{A}^{4}}{64 F_{\pi}^{6}} \frac{\vec{k} \vec{q}_{2} \cdot \vec{\sigma}_{2} \vec{q}_{3} \cdot \vec{\sigma}_{3}\left(\boldsymbol{\tau}_{2} \cdot \tau_{3} \tau_{1}^{a}\left(\vec{q}_{2} \cdot \vec{\sigma}_{1}+\vec{q}_{3} \cdot \vec{\sigma}_{1}\right)-2 \boldsymbol{\tau}_{1} \cdot \tau_{2} \tau_{3}^{a}\left(\vec{q}_{1} \cdot \vec{\sigma}_{1}+\vec{q}_{2} \cdot \vec{\sigma}_{1}\right)\right)}{\left[k^{2}+M_{\pi}^{2}\right]\left[q_{2}^{2}+M_{\pi}^{2}\right]\left[q_{3}^{2}+M_{\pi}^{2}\right]} .
\end{aligned}
$$

The pion-pole terms can be related to the corresponding contributions to the leading four-nucleon force at $\mathrm{N}^{3} \mathrm{LO}$ if we multiply the axial current operator by $-g_{A} /\left(2 F_{\pi}^{2}\right) \overrightarrow{\sigma_{4}} \tau_{4}^{a}$ and replace $\vec{k} \rightarrow-\vec{q}_{4}$ :

$$
\begin{aligned}
V_{\text {class-I }} & =\left.\frac{1}{2} \sum_{a} \tau_{4}^{a} \vec{\sigma}_{4} \cdot \vec{C}_{5}^{a}\right|_{\vec{k}=-\vec{q}_{4}}+23 \text { permutations, } \\
V_{\text {class-II }}^{1} & =\left.\frac{1}{2} \sum_{a} \tau_{4}^{a} \vec{\sigma}_{4} \cdot \vec{C}_{6}^{a}\right|_{\vec{k}=-\vec{q}_{4}}+23 \text { permutations, } \\
V_{\text {class-II }}^{2} & =\left.\frac{1}{4} \sum_{a} \tau_{4}^{a} \vec{\sigma}_{4} \cdot \vec{C}_{7}^{a}\right|_{\vec{k}=-\vec{q}_{4}}+\left.\frac{1}{3} \sum_{a} \tau_{4}^{a} \vec{\sigma}_{4} \cdot \vec{C}_{8}^{a}\right|_{\vec{k}=-\vec{q}_{4}}+23 \text { permutations }
\end{aligned}
$$

where the expressions for the four-nucleon force contributions $V_{\text {class-I }}, V_{\text {class-II }}^{1}$ and $V_{\text {class-II }}^{2}$ are taken from [36].

Finally, in Fig. 21, we show all non-vanishing diagrams which contribute to the $3 \mathrm{~N}$ axial vector current and include one or more insertions of the leading $2 \mathrm{~N}$ contact interactions. These diagrams also do not contribute to the axial charge. All these diagrams yield contributions which depend on the unitary phases:

$$
\begin{aligned}
& (1),(2) \sim 1+\alpha_{1}^{a x}, \\
& (3),(4) \sim \alpha_{1}^{a x},
\end{aligned}
$$

(7), (9), (10), $(13-19) \sim-1+\alpha_{21}^{a x}+\alpha_{22}^{a x}+\alpha_{23}^{a x}$,

$(5),(6) \sim\left(-1+2 \alpha_{4}^{a x}-\alpha_{5}^{a x}+\alpha_{10}^{a x}\right) \frac{1}{\left(k^{2}+M_{\pi}^{2}\right)^{2}}[\ldots]+\left(-1+\alpha_{12}^{a x}\right) \frac{1}{k^{2}+M_{\pi}^{2}}[\ldots]$,

(8) $\sim\left(-1+2 \alpha_{4}^{a x}-\alpha_{5}^{a x}+\alpha_{10}^{a x}\right) \frac{1}{\left(k^{2}+M_{\pi}^{2}\right)^{2}}[\cdots]+\alpha_{12}^{a x} \frac{1}{k^{2}+M_{\pi}^{2}}[\cdots]$,

(12) $\sim-2+\alpha_{25}^{a x}+\alpha_{26}^{a x}$,

(11) $\sim\left(-2+2 \alpha_{4}^{a x}-\alpha_{5}^{a x}+\alpha_{10}^{a x}+\alpha_{21}^{a x}+\alpha_{22}^{a x}+\alpha_{23}^{a x}\right) \frac{1}{\left(k^{2}+M_{\pi}^{2}\right)^{2}}[\ldots]+\alpha_{12}^{a x} \frac{1}{k^{2}+M_{\pi}^{2}}[\ldots]$. 


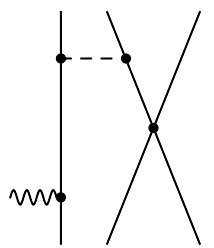

(1)

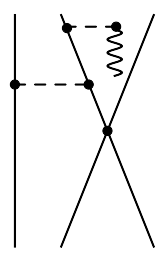

(8)

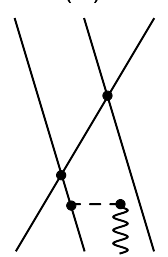

(15)

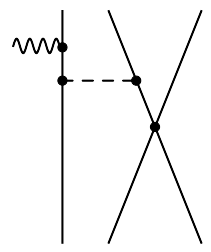

(2)

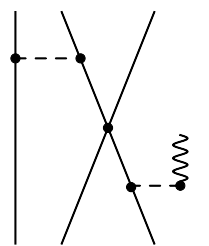

(9)

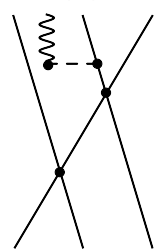

(16)

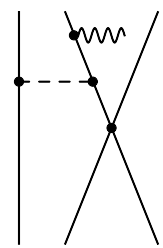

(3)

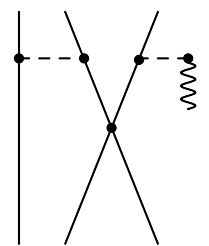

(10)

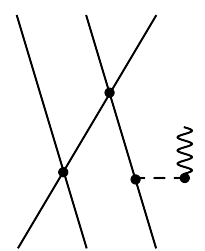

(17)

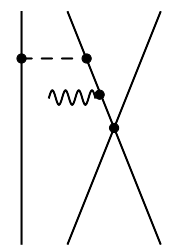

(4)

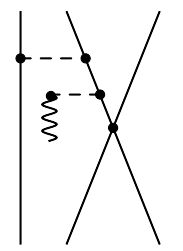

(11)

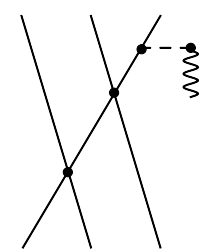

(18)

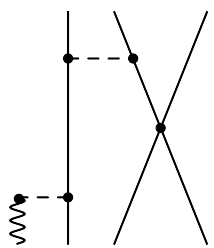

(5)

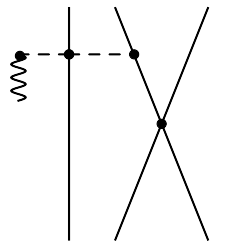

(12)

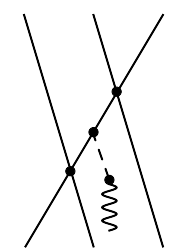

(19)

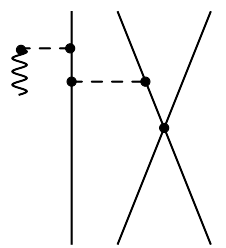

(6)

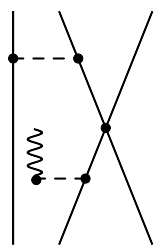

(7)

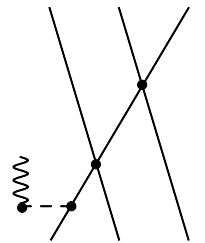

(13)

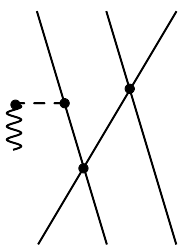

(14)

FIG. 21: Tree-level diagrams involving one or two insertions of the leading contact interactions from $\mathcal{L}_{N N}^{(0)}$ which lead to non-vanishing contributions to $\vec{A}_{3 \mathrm{~N}}^{(Q)}$. For notation see Fig. 10.

For our standard choice of the unitary phases, the contributions of graphs (3), (4), (7-11), (13-19) and all expressions involving second-order pion-pole terms vanish yielding:

$$
\vec{A}_{3 \mathrm{~N}: \text { cont }}^{a(Q)}=-\frac{2 F_{\pi}^{2}}{g_{A}} \sum_{i=1}^{3} \vec{D}_{i}^{a}+5 \text { permutations }
$$

with

$$
\begin{aligned}
\vec{D}_{1}^{a} & =-\frac{g_{A}^{4} C_{T}}{4 F_{\pi}^{4}}\left[\left(\vec{k}-\vec{q}_{1}\right)\left[\boldsymbol{\tau}_{1} \times \boldsymbol{\tau}_{3}\right]^{a}\left(\vec{k} \cdot \vec{\sigma}_{2} \times \vec{\sigma}_{3}-\vec{q}_{1} \cdot \vec{\sigma}_{2} \times \vec{\sigma}_{3}\right)-\left(\tau_{2}^{a}-\tau_{3}^{a}\right)\left(\left(\vec{k}-\vec{q}_{1}\right) \vec{\sigma}_{1} \cdot \vec{\sigma}_{2}\left(\vec{k} \cdot \vec{\sigma}_{3}-\vec{q}_{1} \cdot \vec{\sigma}_{3}\right)\right.\right. \\
& \left.\left.+\vec{\sigma}_{3}\left(\left(\vec{k} \cdot \vec{\sigma}_{1}-\vec{q}_{1} \cdot \vec{\sigma}_{1}\right)\left(\vec{k} \cdot \vec{\sigma}_{2}-\vec{q}_{1} \cdot \vec{\sigma}_{2}\right)+\left(2 \vec{k} \cdot \vec{q}_{1}-k^{2}-q_{1}^{2}\right) \vec{\sigma}_{1} \cdot \vec{\sigma}_{2}\right)\right)\right] \frac{1}{\left[\left(\vec{q}_{1}-\vec{k}\right)^{2}+M_{\pi}^{2}\right]^{2}}, \\
\vec{D}_{2}^{a} & =\frac{g_{A}^{4} C_{T}}{4 F_{\pi}^{4}} \frac{\vec{k}\left(\vec{q}_{1} \cdot \vec{\sigma}_{1} \times \vec{\sigma}_{3}+\vec{q}_{3} \cdot \vec{\sigma}_{1} \times \vec{\sigma}_{3}\right)\left(\tau_{3}^{a} \vec{k} \cdot \vec{q}_{2} \times \vec{\sigma}_{2}+\left[\boldsymbol{\tau}_{2} \times \boldsymbol{\tau}_{3}\right]^{a}\left(k^{2}-\vec{k} \cdot \vec{q}_{2}\right)\right)}{\left[k^{2}+M_{\pi}^{2}\right]\left[\left(\vec{q}_{1}+\vec{q}_{3}\right)^{2}+M_{\pi}^{2}\right]^{2}}, \\
\vec{D}_{3}^{a} & =-\frac{g_{A}^{2} C_{T}}{8 F_{\pi}^{4}} \frac{\vec{k}\left(\vec{q}_{1} \cdot \vec{\sigma}_{1} \times \vec{\sigma}_{3}+\vec{q}_{3} \cdot \vec{\sigma}_{1} \times \vec{\sigma}_{3}\right)\left[\boldsymbol{\tau}_{2} \times \boldsymbol{\tau}_{3}\right]^{a}}{\left[k^{2}+M_{\pi}^{2}\right]\left[\left(\vec{q}_{1}+\vec{q}_{3}\right)^{2}+M_{\pi}^{2}\right]} .
\end{aligned}
$$

The connection to the four-nucleon force manifests itself through the relations

$$
\begin{aligned}
V_{\text {class-IV }} & =\left.\sum_{a} \tau_{4}^{a} \vec{\sigma}_{4} \cdot \vec{D}_{2}^{a}\right|_{\vec{k}=-\vec{q}_{4}}+23 \text { permutations, } \\
V_{\text {class-V }} & =\left.\sum_{a} \tau_{4}^{a} \vec{\sigma}_{4} \cdot \vec{D}_{3}^{a}\right|_{\vec{k}=-\vec{q}_{4}}+23 \text { permutations, }
\end{aligned}
$$

where the expressions for the four-nucleon force contributions $V_{\text {class-IV }}$ and $V_{\text {class-V }}^{2}$ are given in [36]. 
TABLE I: Chiral expansion of the nuclear axial current operator up to $\mathrm{N}^{3} \mathrm{LO}$.

\begin{tabular}{|c|c|c|c|}
\hline order & single-nucleon & two-nucleon & three-nucleon \\
\hline $\mathrm{LO}\left(Q^{-3}\right)$ & $\vec{A}_{1 \mathrm{~N}: \text { static }}^{a}$, Eq. $(4.2)$ & - & - \\
\hline $\operatorname{NLO}\left(Q^{-1}\right)$ & $\vec{A}_{1 \mathrm{~N} \text { : static }}^{a}$, Eq. (4.7) & - & - \\
\hline $\mathrm{N}^{2} \mathrm{LO}\left(Q^{0}\right)$ & - & $\begin{array}{r}\vec{A}_{2 \mathrm{~N}: 1 \pi}^{a} \text {, Eq. }(5.7) \\
+\vec{A}_{2 \mathrm{~N}: \text { cont }}^{a} \text {, Eq. }(5.8)\end{array}$ & - \\
\hline $\mathrm{N}^{3} \mathrm{LO}(Q)$ & $\begin{aligned} & \vec{A}_{1 \mathrm{~N}: \mathrm{static}}^{a}, \\
+\vec{A}_{1 \mathrm{~N}: 1 / m, \mathrm{UT}^{\prime}}^{a}, & \text { Eq. }(4.46) \\
+\vec{A}_{1 \mathrm{~N}: 1 / \mathrm{m}^{2}}^{a}, & \text { Eq. }(4.18)\end{aligned}$ & 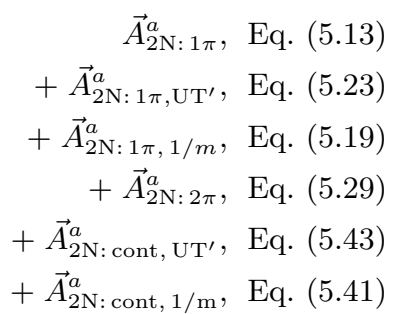 & $\begin{array}{r}\vec{A}_{3 \mathrm{~N}: \pi}^{a}, \text { Eq. }(6.2) \\
+\vec{A}_{3 \mathrm{~N}: \text { cont }}^{a}, \text { Eq. }(6.6)\end{array}$ \\
\hline
\end{tabular}

TABLE II: Chiral expansion of the nuclear axial charge operator up to $\mathrm{N}^{3} \mathrm{LO}$.

\begin{tabular}{|c|c|c|c|}
\hline order & single-nucleon & two-nucleon & three-nucleon \\
\hline $\mathrm{LO}\left(Q^{-3}\right)$ & - & - & - \\
\hline $\operatorname{NLO}\left(Q^{-1}\right)$ & $\begin{array}{r}A_{1 \mathrm{~N}: \mathrm{UT}^{\prime}}^{0, a}, \text { Eq. (4.4) } \\
+A_{1 \mathrm{~N}: 1 / m}^{0, a}, \text { Eq. (4.10) }\end{array}$ & $A_{2 \mathrm{~N}: 1 \pi}^{0, a}$, Eq. (5.3) & - \\
\hline $\mathrm{N}^{2} \mathrm{LO}\left(Q^{0}\right)$ & - & - & - \\
\hline $\mathrm{N}^{3} \mathrm{LO}(Q)$ & 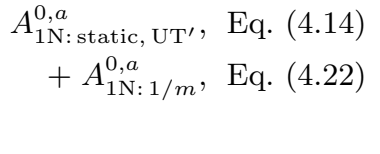 & $\begin{aligned} A_{2 \mathrm{~N}: 1 \pi}^{0, a}, & \text { Eq. }(5.14) \\
+A_{2 \mathrm{~N}: 2 \pi}^{0, a}, & \text { Eq. }(5.30) \\
+A_{2 \mathrm{~N}: \mathrm{cont}}^{0, a}, & \text { Eq. }(5.34)\end{aligned}$ & - \\
\hline
\end{tabular}

\section{SUMMARY OF THE DERIVED CONTRIBUTIONS}

In this section we provide a summary of the derived contributions to the nuclear axial charge and current operators

$$
\boldsymbol{A}^{\mu}=\boldsymbol{A}_{1 \mathrm{~N}}^{\mu}+\boldsymbol{A}_{2 \mathrm{~N}}^{\mu}+\boldsymbol{A}_{3 \mathrm{~N}}^{\mu}+\ldots
$$

The chiral power counting implies that $n$-nucleon operators are, in general, suppressed by two powers of the expansion parameter relative to $n-1$-nucleon operators. Thus, to LO we have:

$$
A_{1 \mathrm{~N}}^{\mu} \sim \mathcal{O}\left(Q^{-3}\right), \quad A_{2 \mathrm{~N}}^{\mu} \sim \mathcal{O}\left(Q^{-1}\right), \quad A_{3 \mathrm{~N}}^{\mu} \sim \mathcal{O}(Q), \quad \ldots
$$

Therefore, up to $\mathrm{N}^{3} \mathrm{LO}(Q)$ in the chiral expansion, i.e. up to order $Q^{4}$ relative to the dominant contribution at order $Q^{-3}$, it is necessary to include single-, two- and three-nucleon operators.

We have worked out all contributions to the nuclear axial current up to $\mathrm{N}^{3} \mathrm{LO}$. The chiral expansion of the current and charge operators is summarized in Tables I and II, respectively. We distinguish explicitly between the static contributions, terms which are proportional to the energy transfer (which are labelled with "UT'") and relativistic corrections $\left(1 / m\right.$ and $\left.1 / m^{2}\right)$. Further, the contributions to the exchange operator are classified according to the range of the interaction between the nucleons (" $1 \pi$ ", " $2 \pi$ " and "cont"). It is important to keep in mind that the expansion 
pattern of the derived operators and their explicit form are, to a large extent, specific to the employed choice of unitary transformations and to the chosen counting scheme for the nucleon mass. To illustrate the importance of consistency between nuclear forces and current operators, we notice that the contributions to the two- and three-nucleon forces and the single- and two-nucleon axial current operators at $\mathrm{N}^{3} \mathrm{LO}$ depend on the phases $\bar{\beta}_{8,9}$, which parametrize the unitary ambiguity of the leading relativistic corrections to the corresponding operators. The approximate independence of the calculated observables on these unitary phases can only be achieved using consistent nuclear forces and current operators.

Last but not least, we would like to comment on isospin-breaking (IB) contributions to the nuclear axial charge and current operators. While we have not explicitly considered IB corrections in our analysis, the only IB contributions to the exchange operators at the considered order within the power counting scheme of Ref. [73] can be accounted for by simply using the proper pion masses in the propagator of the leading $2 \mathrm{~N}$ charge operator $A_{2 \mathrm{~N}: 1 \pi}^{0, a}\left(Q^{-1}\right)$ in Eq. $(5.3)$. IB corrections to the $2 \mathrm{~N}$ current operator start contributing at order $Q^{2}$ which is not considered in our work.

\section{PSEUDOSCALAR CURRENTS}

Chiral symmetry connects different physical processes via chiral Ward identities. In particular, the axial-vector current is connected to pseudoscalar current via the continuity equation (2.42). Thus, the pseudoscalar current can be determined (up to unitary ambiguity) from the axial current using Eq. (2.42). Instead of following this approach, we use chiral EFT in combination with the MUT as explained in the previous sections to independently derive the pseudoscalar current up-to-and-including order $Q^{4}$ relative to the dominant one-body term. This will allow us to perform a highly nontrivial check of our results by explicitly verifying the validity of the continuity equation (2.42). Notice that the derived expressions might also be useful in connection with ongoing and future direct-detection experiments of weakly-interacting massive particles (WIMP) through scattering off nuclei, see Ref. [74] for a related discussion.

Throughout this section, we follow the notation of Ref. [61] and introduce a LEC $G_{\pi}$ defined as

$$
\left\langle 0\left|\bar{q} i \gamma_{5} \tau^{a} q\right| \pi^{b}\right\rangle=\delta_{a b} G_{\pi},
$$

and related to the physical pion mass via

$$
F_{\pi} M_{\pi}^{2}=m_{q} G_{\pi}
$$

Notice that the current operators describing nuclear response on external pseudoscalar source which couples to the QCD density $\bar{q} i \gamma_{5} \tau^{a} q$ are renormalization-scale dependent. This manifests itself in all resulting expressions for the current operator being proportional to the LEC $G_{\pi}$, whose value depends on the choice of renormalization conditions in QCD. Notice that in the continuity equation, pseudoscalar current operators always occur in a renormalizationscale invariant combination with the quark mass $m_{q}$, see Eqs. (2.42) and (8.2). As an alternative convention, one could directly absorb the quark mass into the definition of the pseudoscalar quark density. This would result in renormalization-scale invariant expressions for the pseudoscalar operators, which can be obtained from our expressions by making the replacement $G_{\pi} \rightarrow F_{\pi} M_{\pi}^{2}$ and shifting the contributions two orders higher in the chiral expansion. In the following we will express all pseudoscalar currents in terms of the quark mass $m_{q}$.

The diagrammatic structure of the various contributions to the pseudoscalar current is the same as in the calculation of the axial-vector current. For this reason we can use the set of diagrams introduced in sections IV-VI, with axial-vector sources being replaced by pseudoscalar ones, also for the calculation of the pseudoscalar current. Notice, however, that certain vertices contribute to the axial-vector current but vanish when the axial-vector source is replaced by the pseudoscalar one. This leads to a simplification such that only a subset of diagrams discussed in the previous sections will have to be considered. The same statement is also valid for contributions involving additional unitary transformations. In order to write down the most general set of additional unitary transformations with a pseudoscalar source we simply take the unitary transformations listed in Appendix B and replace all vertices involving axial-vector sources by the corresponding ones with pseudoscalar sources

$$
A_{a, b}^{\left(\kappa_{i}\right)} \rightarrow P_{a, b}^{\left(\kappa_{j}\right)}
$$

where $P_{a, b}^{\left(\kappa_{j}\right)}$ denotes an interaction from the Hamiltonian with one pseudoscalar current, $a$ nucleons, $b$ pion fields and $\kappa_{j}$ defined by Eq. (2.10). Since the unitary phases of the additional unitary transformations with pseudoscalar sources 
may, in general, be different from the unitary phases of the similar transformations with axial vector sources, we also make a replacement

$$
\alpha_{i}^{a x} \rightarrow \alpha_{i}^{p}
$$

in Eq. (B.4) and consider $\alpha_{i}^{p}$ as independent parameters. By this procedure we obtain, for example,

$$
S_{1}^{a x}=\alpha_{1}^{a x} \eta A_{2,0}^{(0)} \eta H_{2,1}^{(1)} \lambda^{1} \frac{1}{E_{\pi}^{3}} H_{2,1}^{(1)} \eta \rightarrow S_{1}^{p}=\alpha_{1}^{p} \eta P_{2,0}^{(0)} \eta H_{2,1}^{(1)} \lambda^{1} \frac{1}{E_{\pi}^{3}} H_{2,1}^{(1)} \eta,
$$

for the first generator of the unitary transformation from Eq. (B.4). Similar replacements are performed for all transformations of Appendix B. In this way, we end up with 33 additional phases which we fix by applying the same requirements as in the case of the axial-vector case. There is, however, a simplification due to the absence of certain interactions with pseudoscalar sources, namely

$$
P_{2,0}^{(0)}=P_{2,1}^{(1)}=P_{2,2}^{(2)}=0,
$$

which implies

$$
S_{1}^{p}=S_{2}^{p}=S_{3}^{p}=0
$$

For this reason, we only need to consider 30 unitary phases $\alpha_{i}^{p}$ with $i=4, \ldots, 15,17, \ldots, 30, \alpha_{16}^{p, \text { LO }}, \alpha_{16}^{p \text {, Static }}, \alpha_{16}^{p, 1 / m}$ and $\alpha_{16}^{p \text {, Tadpole }}$.

\section{A. Unitary phases of pseudoscalar transformations}

To fix the values of the additional unitary transformations involving a pseudoscalar source, we proceed as in the case of the axial vector current:

1. We require the one-loop contributions to the pseudoscalar current to be expressible in the form of 4-dimensional integrals with heavy-baryon propagators. This requirement is necessary for factorizability of the exchanged pions which is necessary (but not sufficient) for the renormalization of the pseudoscalar current. It leads to the following 7 constraints

$$
\begin{aligned}
\alpha_{8}^{p} & =\alpha_{5}^{p}, \\
\alpha_{9}^{p} & =-\alpha_{4}^{p}+\alpha_{5}^{p}+\alpha_{6}^{p}-\alpha_{7}^{p}, \\
\alpha_{14}^{p} & =\alpha_{4}^{p}-\alpha_{11}^{p}, \\
\alpha_{27}^{p} & =\alpha_{25}^{p}, \\
\alpha_{28}^{p} & =\alpha_{25}^{p}-\alpha_{26}^{p}, \\
\alpha_{29}^{p} & =\alpha_{25}^{p}-\alpha_{26}^{p}, \\
\alpha_{30}^{p} & =-\alpha_{26}^{p} .
\end{aligned}
$$

2. The resulting expressions for the pseudoscalar current operators are required to be properly renormalized. However, we found that this requirement does not yield any further constraints on the pseudoscalar unitary phases.

3. We require the pion-pole part of the pseudoscalar current to match the corresponding expressions for the nuclear forces upon replacing the leading pseudoscalar source-pion interaction by the leading pion-nucleon vertex. Matching the two-pion-exchange current to the corresponding two-pion-one-pion contributions of $\mathrm{N}^{3} \mathrm{LO}$ $3 \mathrm{NF}$ gives three constraints

$$
\begin{aligned}
\alpha_{13}^{p} & =2+\alpha_{5}^{p}+\alpha_{6}^{p}-\alpha_{10}^{p}-\alpha_{11}^{p}-\alpha_{14}^{p} \\
\alpha_{15}^{p} & =-\alpha_{12}^{p} \\
\alpha_{26}^{p} & =1-\alpha_{25}^{p} .
\end{aligned}
$$


Finally, matching the $1 / m$-corrections of the pseudoscalar current with the corresponding $1 / m$-corrections to the $\mathrm{N}^{3} \mathrm{LO} 3 \mathrm{NFs}$ yields the last three constraints:

$$
\begin{aligned}
\alpha_{20}^{p} & =-1-2 \bar{\beta}_{8}+\alpha_{17}^{p}+\alpha_{18}^{p}+\alpha_{19}^{p}, \\
\alpha_{24}^{p} & =-2+\alpha_{21}^{p}+\alpha_{22}^{p}+\alpha_{23}^{p}, \\
\alpha_{16}^{p, 1 / m} & =-\frac{1}{2}\left(1+2 \bar{\beta}_{9}\right) .
\end{aligned}
$$

Matching the pion-pole contributions to the three-nucleon pseudoscalar current operator with the corresponding terms in the four-nucleon force does not produce any additional constraints. The same is true for the singlenucleon current operator.

With these constraints, the pseudoscalar current has no further unitary ambiguity and becomes unique (modulo the phases $\bar{\beta}_{8}$ and $\left.\bar{\beta}_{9}\right)$ at the considered chiral order. Furthermore, we have verified that the pion-pole contributions to the single-nucleon (two-nucleon) pseudoscalar current operator proportional to the energy transfer $k_{0}$ match the corresponding terms in the two-nucleon (three-nucleon) force for the choice of $\bar{\beta}_{9}=1 / 2\left(\bar{\beta}_{8}=1 / 2\right)$. Here and in what follows, Eqs. (8.8), (8.9) and (8.10) are referred to as the standard choice of the unitary phases for the pseudoscalar current operator.

We are now in the position to present explicit results for the pseudoscalar nuclear currents up to fourth order in the chiral expansion. Due to the direct relations between pion pole contributions to the pseudoscalar and axial-vector current as described below, the corresponding matching relations with the nuclear forces can be taken from sections $\mathrm{V}$, VI and will not be given explicitly in the following.

\section{B. Single-nucleon pseudoscalar current operator}

The single-nucleon pseudoscalar current starts to contribute at order $Q^{-4}$. The dominant contribution emerges from diagram (2) of Fig. 2. At this order, there is no dependence on the unitary phases. The leading-order expression for the pseudoscalar current is given by

$$
P_{1 \mathrm{~N}: \text { static }}^{a\left(Q^{-4}\right)}=i \frac{M_{\pi}^{2} g_{A}}{2 m_{q}} \tau_{i}^{a} \frac{\vec{k} \cdot \vec{\sigma}_{i}}{k^{2}+M_{\pi}^{2}}=-i \frac{1}{m_{q}} \vec{k} \cdot \vec{A}_{1 \mathrm{~N}: \text { static }}^{a\left(Q^{-3}\right)},
$$

with $\vec{A}_{1 \mathrm{~N}: \text { static }}^{a\left(Q^{-3}\right)}$ given in Eq. $(4.2)$.

At order $Q^{-3}$, there are only vanishing contributions, i.e.

$$
P_{1 \mathrm{~N}: \text { static }}^{a\left(Q^{-3}\right)}=0
$$

Next, at order $Q^{-2}$, we have to account for two kinds of corrections. The first one comes from the leading oneloop contributions, along with the corresponding counter-terms from the $\mathcal{L}_{\pi N}^{(3)}$ Lagrangian. One-loop diagrams that contribute to the pseudoscalar current at order $Q^{-2}$ are shown in Fig. 3. Only diagrams (3) and (4) yield nonvanishing contributions. There is no explicit dependence on unitary phases for pseudoscalar current at this order. Non-vanishing counterterms and tadpoles are given by diagrams $(1-4)$ and $(6-8)$ of Fig. 4 . None of the resulting contributions depend on unitary phases. The final result for the pseudoscalar current operator at order $Q^{-2}$ in the static limit is given by

$$
P_{1 \mathrm{~N}: \text { static }}^{a\left(Q^{-2}\right)}=i \frac{M_{\pi}^{2} \bar{d}_{18}}{m_{q}} k^{2} \tau_{i}^{a} \frac{\vec{k} \cdot \vec{\sigma}_{i}}{k^{2}+M_{\pi}^{2}}=-i \frac{1}{m_{q}} \vec{k} \cdot \vec{A}_{1 \mathrm{~N}: \text { static }}^{a}\left(Q^{-1}\right)
$$

with $\vec{A}_{1 \mathrm{~N} \text { : static }}^{a\left(Q^{-1}\right)}$ given in Eq. (4.7).

The second kind of contributions at order $Q^{-2}$ could emerge from relativistic $1 / m$-corrections. However, the corresponding diagrams shown in Fig. 5 only generate operators which are proportional to $k_{0}$ and thus do not contribute 
at order $Q^{-2}$. More precisely, the non-vanishing contributions are generated by diagrams (2) and (3) of Fig. 5. Their unitary phase dependence is given by

$$
\begin{aligned}
& (2) \sim \frac{k_{0}}{m} \frac{\alpha_{17}^{p}+\alpha_{18}^{p}+\alpha_{19}^{p}-\alpha_{20}^{p}}{\left(k^{2}+M_{\pi}^{2}\right)^{2}}, \\
& (3) \sim \frac{k_{0}}{m} \frac{\alpha_{16}^{p, 1 / m}}{k^{2}+M_{\pi}^{2}} .
\end{aligned}
$$

Given that these terms contribute at order $Q^{0}$, we find

$$
P_{1 \mathrm{~N}: 1 / m}^{a\left(Q^{-2}\right)}=0
$$

At order $Q^{-1}$, there are no $c_{i} / m$-corrections and no $k_{0}$-dependent contributions to the $1 \mathrm{~N}$ pseudoscalar current, which can be traced back to vanishing $Q^{-3}$ terms. We also found only vanishing contributions from one-loop diagrams with a single insertion of subleading vertices (after renormalization). Thus, there are no contributions to the single-nucleon pseudoscalar current at order $Q^{-1}$.

Finally there are various corrections at order $Q^{0}$. We begin with terms proportional to $k_{0} / m$ which emerge from diagrams (2) and (3) shown in Fig. 5. Their unitary phase dependence is given by Eq. (8.14), and the explicit expressions for the standard choice of the unitary phases are given by

$$
P_{1 \mathrm{~N}: 1 / m, \mathrm{UT}^{\prime}}^{a\left(Q^{0}\right)}=i \frac{M_{\pi}^{2}}{m_{q} k^{2}} \vec{k} \cdot \vec{A}_{1 \mathrm{~N}: 1 / m, \mathrm{UT}^{\prime}}^{a},
$$

with $\vec{A}_{1 \mathrm{~N}: 1 / m, \mathrm{UT}^{\prime}}^{a}$ given in Eq. (4.13).

The second kind of corrections could emerge from static terms proportional to the energy transfer $k_{0}$. We, however, find a vanishing result for such contributions.

The third kind of order- $Q^{0}$ contributions is given by relativistic $1 / m^{2}$-corrections. The corresponding diagrams yielding non-vanishing results are (2), (3), (4) and (5) of Fig. 6. The unitary phase dependence of these graphs is given by

$$
\begin{aligned}
(2) & \sim 1+\alpha_{16}^{p, 1 / m} \\
(3),(4) & \sim 2-\alpha_{17}^{p}-\alpha_{18}^{p}-\alpha_{19}^{p}+\alpha_{20}^{p} .
\end{aligned}
$$

The contribution of diagram (5) does not depend on unitary phases. Our final result for $1 / \mathrm{m}^{2}$-corrections at order $Q^{0}$ for the standard choice of the phases is given by

$$
P_{1 \mathrm{~N}: 1 / \mathrm{m}^{2}}^{a\left(Q^{0}\right)}=i \frac{M_{\pi}^{2} g_{A}}{16 m_{q} m^{2}} \tau_{i}^{a}\left(\vec{k} \cdot \vec{\sigma}_{i}\left(1-2 \bar{\beta}_{8}\right) \frac{\left(p_{i}^{\prime 2}-p_{i}^{2}\right)^{2}}{\left(k^{2}+M_{\pi}^{2}\right)^{2}}-2 \frac{\left(p_{i}^{\prime 2}+p_{i}^{2}\right) \vec{k} \cdot \vec{\sigma}_{i}-2 \bar{\beta}_{9}\left(p_{i}^{\prime 2}-p_{i}^{2}\right) \vec{k}_{i} \cdot \vec{\sigma}_{i}}{k^{2}+M_{\pi}^{2}}\right)
$$

Notice that this expression is related to the pion-pole terms in the corresponding axial current operator $\vec{A}_{1 \mathrm{~N}: 1 / \mathrm{m}^{2}}^{a}$, whose expression is given in Eq. (4.18), via

$$
P_{1 \mathrm{~N}: 1 / \mathrm{m}^{2}}^{a\left(Q^{0}\right)}=\left.i \frac{M_{\pi}^{2}}{m_{q} k^{2}} \vec{k} \cdot \vec{A}_{1 \mathrm{~N}: 1 / \mathrm{m}^{2}}^{a(Q)}\right|_{\text {pion-pole terms }} .
$$

The fourth kind of order- $Q^{0}$ contributions are coming from relativistic $1 / m$-corrections to the leading one-loop current. The corresponding non-vanishing diagrams are (2) and (3) of Fig. 7, and all diagrams of Fig. 9. The unitary phase dependence of these diagrams is given by

$$
\begin{aligned}
(2) & \sim 1+\alpha_{16}^{p, \text { Static }}, \\
(21) & \sim 1+2 \bar{\beta}_{9}, \\
(22) & \sim 1-2 \bar{\beta}_{9},
\end{aligned}
$$


(23) $\sim 1+\alpha_{16}^{p, 1 / m}$

$(24) \sim\left(1+2 \bar{\beta}_{8}-\alpha_{12}^{p}-\alpha_{15}^{p}\right) \frac{1}{k^{2}+M_{\pi}^{2}}[\ldots]+\left(2-\alpha_{4}^{p}+\alpha_{5}^{p}+\alpha_{6}^{p}-\alpha_{10}^{p}-\alpha_{13}^{p}\right) \frac{1}{\left(k^{2}+M_{\pi}^{2}\right)^{2}}[\cdots]$,

$(26) \sim\left(\alpha_{12}^{p}+\alpha_{15}^{p}\right) \frac{1}{k^{2}+M_{\pi}^{2}}[\ldots]+\left(4-\alpha_{4}^{p}+\alpha_{5}^{p}+\alpha_{6}^{p}-\alpha_{10}^{p}-\alpha_{13}^{p}-\alpha_{17}^{p}-\alpha_{18}^{p}-\alpha_{19}^{p}+\alpha_{20}^{p}\right) \frac{1}{\left(k^{2}+M_{\pi}^{2}\right)^{2}}[\ldots]$,

$(27) \sim\left(1-2 \bar{\beta}_{8}\right) \frac{1}{k^{2}+M_{\pi}^{2}}[\ldots]+\left(2-\alpha_{17}^{p}-\alpha_{18}^{p}-\alpha_{19}^{p}+\alpha_{20}^{p}\right) \frac{1}{\left(k^{2}+M_{\pi}^{2}\right)^{2}}[\ldots]$,

while the remaining graphs do not depend on the unitary phases. For our standard choice of the phases we get a vanishing result after renormalization,

$$
P_{1 \mathrm{~N}: 1 / \mathrm{m}}^{a\left(Q^{0}\right)}=0
$$

Before specifying the last missing kind of corrections emerging from static contributions at the two-loop level, it is instructive to compare our results with the on-shell expressions for pseudoscalar amplitude, which can be written in terms of the form factor $G_{\pi N}(t)$ as follows

$$
\begin{aligned}
\left\langle N\left(p_{i}^{\prime}\right)\left|P^{a}(0)\right| N\left(p_{i}\right)\right\rangle & \equiv\left\langle N\left(p_{i}^{\prime}\right)\left|\bar{q} i \gamma_{5} \tau^{a} q\right| N\left(p_{i}\right)\right\rangle=: \frac{G_{\pi}}{M_{\pi}^{2}-t} G_{\pi N}(t) \frac{1}{2 m} \bar{u}\left(p_{i}^{\prime}\right) i \gamma_{5} \tau_{i}^{a} u\left(p_{i}\right) \\
& =i \tau_{i}^{a} \frac{G_{\pi}}{2 m} \frac{G_{\pi N}(t)}{M_{\pi}^{2}-t}\left(-\vec{k} \cdot \vec{\sigma}_{i}+\mathcal{O}\left(1 / m^{2}\right)\right) .
\end{aligned}
$$

The second equation in the first line of (8.22) serves as a definition of the form factor $G_{\pi N}(t)$, which for a particular choice of pion interpolating fields can be identified with the pion-nucleon form factor. As it is well known, the form factor $G_{\pi N}(t)$ is related to the axial and pseudoscalar form factors $G_{A}(t)$ and $G_{P}(t)$ via the Partially Conserved Axial Current $(\mathrm{PCAC})$ relation:

$$
2 m G_{A}(t)+\frac{t}{2 m} G_{P}(t)=2 \frac{M_{\pi}^{2} F_{\pi}}{M_{\pi}^{2}-t} G_{\pi N}(t) .
$$

Using this relation along with Eqs. (4.27) and (4.34), we can read off the chiral expansion of the form-factor $G_{\pi N}(t)$ :

$$
G_{\pi N}(t)=\frac{m}{F_{\pi}}\left(g_{A}-2 \bar{d}_{18} t+\left[1-\frac{t}{M_{\pi}^{2}}\right]\left[G_{A}^{\left(Q^{4}\right)}(t)+\frac{t}{4} G_{P}^{\left(Q^{2}\right)}(t)\right]\right)+\mathcal{O}\left(Q^{5}\right) .
$$

Notice that since

$$
G_{A}^{\left(Q^{4}\right)}(t)+\frac{t}{4} G_{P}^{\left(Q^{2}\right)}(t)=M_{\pi}^{2} f(t)
$$

where the function $f(t)$ is finite in the chiral limit, the form factor $G_{\pi N}(t)$ is clearly well-defined in the chiral limit. Plugging Eq. (8.24), truncated at order $Q^{4}$, into the parametrization (8.22), we read off the on-shell expression for the static contribution to the single-nucleon pseudoscalar current operator $P_{1 \mathrm{~N} \text { : static }}^{a\left(Q^{0}\right)}$ from the relation

$$
\left\langle N\left(p_{i}^{\prime}\right)\left|P^{a}(0)\right| N\left(p_{i}\right)\right\rangle+P_{1 \mathrm{~N}: \text { static }}^{a\left(Q^{-4}\right)}+P_{1 \mathrm{~N}: \text { static }}^{a\left(Q^{-2}\right)}+P_{1 \mathrm{~N}: \text { static }}^{a\left(Q^{0}\right)}=\mathcal{O}\left(1 / m^{2}\right)
$$

to be

$$
P_{1 \mathrm{~N}: \text { static }}^{a\left(Q^{0}\right)}=i \frac{1}{8 m_{q}} \vec{k} \cdot \vec{\sigma}_{i} \tau_{i}^{a}\left(4 G_{A}^{\left(Q^{4}\right)}\left(-k^{2}\right)-k^{2} G_{P}^{\left(Q^{2}\right)}\left(-k^{2}\right)\right)
$$

As in the case of the axial-vector current, we conjecture that the static contribution to the single-nucleon pseudoscalar current calculated using the MUT at the two-loop level coincides with the above on-shell expression.

It is also instructive to verify the consistency between our results for the current operator and the parametrization (8.22) for $1 / \mathrm{m}^{2}$-corrections. Taking into account the nonrelativistic normalization of the nucleon states in the expressions for the current, we obtain the relation

$$
\left[\frac{G_{\pi}}{M_{\pi}^{2}-t} G_{\pi N}(t) \frac{1}{2 m} \sqrt{\frac{m}{E_{p_{i}^{\prime}}}} \bar{u}\left(p_{i}^{\prime}\right) i \gamma_{5} \tau_{i}^{a} u\left(p_{i}\right) \sqrt{\frac{m}{E_{p_{i}}}}\right]_{1 / m^{2}-\mathrm{part}}+P_{1 \mathrm{~N}: 1 / m^{2}}^{a(Q)}+P_{1 \mathrm{~N}: \mathrm{UT}^{\prime}}^{a(Q)}
$$




$$
=-i\left(k_{0}-\frac{p_{i}^{\prime 2}}{2 m}+\frac{p_{i}^{2}}{2 m}\right) \frac{g_{A} M_{\pi}^{2}}{8 m_{q} m} \frac{1}{k^{2}+M_{\pi}^{2}} \tau_{i}^{a}\left[-\left(1+2 \bar{\beta}_{8}\right)\left(p_{i}^{\prime 2}-p_{i}^{2}\right) \frac{\vec{k} \cdot \vec{\sigma}_{i}}{k^{2}+M_{\pi}^{2}}+2\left(1+2 \bar{\beta}_{9}\right) \vec{k}_{i} \cdot \vec{\sigma}_{i}\right] .
$$

As expected, we observe that our results for the current operator agree with the parametrization (8.22) on the energy shell, i.e. with $k_{0}=p_{i}^{\prime 2} / 2 m-p_{i}^{2} / 2 m$. As in the case of the axial-vector current, the two expressions agree even off the energy shell for a particular choice of the phases $\bar{\beta}_{8,9}$, namely $\bar{\beta}_{8}=\bar{\beta}_{9}=-1 / 2$.

To conclude, to order $Q^{0}$, the single nucleon pseudoscalar current operator can be written in terms of the axial and and induced pseudoscalar form factors of the nucleon as

$$
P_{1 \mathrm{~N}}^{a}=i \frac{1}{2 m_{q}} \tau_{i}^{a} \vec{k} \cdot \vec{\sigma}_{i}\left(G_{A}\left(-k^{2}\right)-\frac{k^{2}}{4 m^{2}} G_{P}\left(-k^{2}\right)\right)+P_{1 \mathrm{~N}: 1 / m, \mathrm{UT}^{\prime}}^{a\left(Q^{0}\right)}+P_{1 \mathrm{~N}: 1 / \mathrm{m}^{2}}^{a\left(Q^{0}\right)},
$$

where the expressions for the last two terms are given in Eqs. (8.16), and (8.18). As already pointed out above, $P_{1 \mathrm{~N}}^{a}$ is well-defined in the chiral limit (except at the kinematical point $k=0$ ).

\section{Two-nucleon pseudoscalar operators}

\section{Contributions at orders $Q^{-2}$ and $Q^{-1}$}

We now turn to the two-nucleon contributions to the pseudoscalar current operator, whose chiral expansion is expected to start at order $Q^{-2}$. The relevant diagrams are (3), (4) and (5) of Fig. 10. All of them yield contributions which depend on the unitary phases:

$$
\begin{aligned}
& (3) \sim k_{0}\left(\alpha_{25}^{p}+\alpha_{26}^{p}\right), \\
& (4) \sim \frac{k_{0}\left(\alpha_{12}^{p}+\alpha_{15}^{p}\right)}{k^{2}+M_{\pi}^{2}}[\ldots]+\frac{k_{0}\left(\alpha_{4}^{p}-\alpha_{5}^{p}-\alpha_{6}^{p}+\alpha_{10}^{p}+\alpha_{13}^{p}\right)}{\left(k^{2}+M_{\pi}^{2}\right)^{2}}[\ldots], \\
& (5) \sim k_{0}\left(\alpha_{21}^{p}+\alpha_{22}^{p}+\alpha_{23}^{p}-\alpha_{24}^{p}\right) .
\end{aligned}
$$

The resulting expressions are proportional to the energy transfer $k_{0}$, so that their actual contributions are shifted to order $Q^{0}$. For this reason, we find a vanishing result for both the one-pion-exchange and contact interaction $2 N$ currents at the order $Q^{-2}$ :

$$
P_{2 \mathrm{~N}: 1 \pi}^{a\left(Q^{-2}\right)}=P_{2 \mathrm{~N}: \text { cont }}^{a\left(Q^{-2}\right)}=0
$$

At order $Q^{-1}$, one encounters various contributions to the $2 \mathrm{~N}$ pseudoscalar current operator stemming from diagrams shown in the second line of Fig. 10, whose explicit expressions have the form

$$
\begin{aligned}
& P_{2 \mathrm{~N}: 1 \pi}^{a\left(Q^{-1}\right)}=-i \frac{1}{m_{q}} \vec{k} \cdot \vec{A}_{2 \mathrm{~N}: 1 \pi}^{a\left(Q^{0}\right)}, \\
& P_{2 \mathrm{~N}: \mathrm{cont}}^{a\left(Q^{-1}\right)}=-i \frac{1}{m_{q}} \vec{k} \cdot \vec{A}_{2 \mathrm{~N}: \mathrm{cont}}^{a\left(Q^{0}\right)},
\end{aligned}
$$

with $\vec{A}_{2 \mathrm{~N}: 1 \pi}^{a\left(Q^{0}\right)}$ and $\vec{A}_{2 \mathrm{~N}: \text { cont }}^{a\left(Q^{0}\right)}$ given in Eqs. (5.7) and (5.8), respectively.

\section{One-pion-exchange contributions at order $Q^{0}$}

We consider first one-loop graphs of non-tadpole type which are visualized in Fig. 11. For the pseudoscalar current, only diagrams $(9-14),(21-22)$ and $(25-27)$ generate nonvanishing contributions, from which those of diagrams $(9-13)$ turn out to depend on the unitary phases:

$$
(9),(10),(13) \sim\left(2-\alpha_{4}^{p}+\alpha_{5}^{p}+\alpha_{6}^{p}-\alpha_{10}^{p}-\alpha_{13}^{p}\right) \frac{1}{\left(k^{2}+M_{\pi}^{2}\right)^{2}}[\ldots]+\left(2-\alpha_{12}^{p}-\alpha_{15}^{p}\right) \frac{1}{k^{2}+M_{\pi}^{2}}[\ldots],
$$




$$
\text { (11), (12) (2- } \left.\alpha_{4}^{p}+\alpha_{5}^{p}+\alpha_{6}^{p}-\alpha_{10}^{p}-\alpha_{13}^{p}\right) \frac{1}{\left(k^{2}+M_{\pi}^{2}\right)^{2}}[\ldots]+\left(\alpha_{12}^{p}+\alpha_{15}^{p}\right) \frac{1}{k^{2}+M_{\pi}^{2}}[\ldots] .
$$

Furthermore, all one-pion-exchange tadpole diagrams and tree graphs involving $d_{i}$-vertices from $\mathcal{L}_{\pi N}^{(3)}$ and $l_{i}$-vertices from $\mathcal{L}_{\pi}^{(4)}$ from Fig. 12 lead to vanishing contributions. After renormalization of the LECs, we find our final result for the static order- $Q^{0}$ contributions to the $2 \mathrm{~N}$ one-pion-exchange pseudoscalar current operator for our standard choice of unitary phases:

$$
P_{2 \mathrm{~N}: 1 \pi}^{a\left(Q^{0}\right)}=i \frac{4 M_{\pi}^{2} F_{\pi}^{2}}{g_{A} m_{q}} \frac{\vec{q}_{1} \cdot \vec{\sigma}_{1}}{q_{1}^{2}+M_{\pi}^{2}}\left\{\tau_{1}^{a}\left[h_{1}^{P}\left(q_{2}\right)+\frac{h_{4}\left(q_{2}\right)}{k^{2}+M_{\pi}^{2}}\right]+\left[\boldsymbol{\tau}_{1} \times \boldsymbol{\tau}_{2}\right]^{a} \vec{q}_{1} \cdot\left[\vec{q}_{2} \times \vec{\sigma}_{2}\right] \frac{h_{5}\left(q_{2}\right)}{k^{2}+M_{\pi}^{2}}\right\}+1 \leftrightarrow 2
$$

where the scalar functions $h_{4}\left(q_{2}\right)$ and $h_{5}\left(q_{2}\right)$ are defined in Eq. (5.15), while $h_{1}^{P}\left(q_{2}\right)$ is given by

$$
h_{1}^{P}\left(q_{2}\right)=\frac{g_{A}^{4}}{256 \pi F_{\pi}^{6}}\left(\left(1-2 g_{A}^{2}\right) M_{\pi}+\left(2 M_{\pi}^{2}+q_{2}^{2}\right) A\left(q_{2}\right)\right) .
$$

It is easy to verify that the pion-pole contributions match the corresponding expressions in the $\mathrm{N}^{3} \mathrm{LO} 3 \mathrm{NF}$. Notice further the relation between the pion-pole terms in the pseudoscalar and the corresponding axial current operator:

$$
\left.P_{2 \mathrm{~N}: 1 \pi}^{a\left(Q^{0}\right)}\right|_{\text {pion-pole terms }}=\left.i \frac{M_{\pi}^{2}}{m_{q} k^{2}} \vec{k} \cdot \vec{A}_{2 \mathrm{~N}: 1 \pi}^{a(Q)}\right|_{\text {pion-pole terms }},
$$

where $A_{2 \mathrm{~N}: 1 \pi}^{a(Q)}$ is specified in Eq. (5.13).

Finally, apart from the static contributions, we need to account for the leading relativistic corrections emerging from tree-level diagrams with a single insertion of $1 / m$-vertices from the Lagrangian $\mathcal{L}_{\pi N}^{(2)}$. From the corresponding diagrams shown in Fig. 13 , only graphs $(8-18)$ yield nonvanishing contributions. Furthermore, diagrams $(8-17)$ turn out to induce the dependence on the unitary phases in the following way:

$$
\begin{aligned}
(8),(9),(10) & \sim\left(-2+\alpha_{4}^{p}-\alpha_{5}^{p}-\alpha_{6}^{p}+\alpha_{10}^{p}+\alpha_{13}^{p}\right) \frac{1}{\left(k^{2}+M_{\pi}^{2}\right)^{2}}[\ldots]+\left(-1-2 \bar{\beta}_{8}+\alpha_{12}^{p}+\alpha_{15}^{p}\right) \frac{1}{k^{2}+M_{\pi}^{2}}[\ldots] \\
(11) & \sim\left(-4+\alpha_{4}^{p}-\alpha_{5}^{p}-\alpha_{6}^{p}+\alpha_{10}^{p}+\alpha_{13}^{p}+\alpha_{17}^{p}+\alpha_{18}^{p}+\alpha_{19}^{p}-\alpha_{20}^{p}\right) \frac{1}{\left(k^{2}+M_{\pi}^{2}\right)^{2}}[\ldots] \\
& +\left(\alpha_{12}^{p}+\alpha_{15}^{p}\right) \frac{1}{k^{2}+M_{\pi}^{2}}[\ldots] \\
(12) & \sim\left(-2+\alpha_{17}^{p}+\alpha_{18}^{p}+\alpha_{19}^{p}-\alpha_{20}^{p}\right) \frac{1}{\left(k^{2}+M_{\pi}^{2}\right)^{2}}[\cdots]+\left(-1+2 \bar{\beta}_{8}\right) \frac{1}{k^{2}+M_{\pi}^{2}}[\ldots] \\
(13) & \sim 1+2 \bar{\beta}_{9}, \\
(14) & \sim 1-2 \bar{\beta}_{9}, \\
(15) & \sim 1+\alpha_{16}^{p, 1 / m}, \\
(16) & \sim-1+\alpha_{25}^{p}+\alpha_{26}^{p}, \\
(17) & \sim-2+\alpha_{25}^{p}+\alpha_{26}^{p} .
\end{aligned}
$$

Again, our standard choice of the unitary phases leads to certain simplifications. In particular, it eliminates the contributions from diagram (16). The explicit result for the $1 / m$-corrections to the one-pion-exchange pseudoscalar NN current operator has the form

$$
\begin{aligned}
P_{2 \mathrm{~N}: 1 \pi, 1 / m}^{a\left(Q^{0}\right)} & =i \frac{g_{A} M_{\pi}^{2}}{16 m_{q} F_{\pi}^{2} m}\left\{i\left[\boldsymbol{\tau}_{1} \times \boldsymbol{\tau}_{2}\right]^{a}\left[-\frac{\vec{B}_{1} \cdot \vec{k}}{\left(q_{1}^{2}+M_{\pi}^{2}\right)^{2}\left(k^{2}+M_{\pi}^{2}\right)}+\frac{1}{q_{1}^{2}+M_{\pi}^{2}}\left(\frac{\overrightarrow{B_{2}} \cdot \vec{k}}{k^{2}\left(k^{2}+M_{\pi}^{2}\right)^{2}}+\frac{\vec{B}_{3} \cdot \vec{k}}{k^{2}\left(k^{2}+M_{\pi}^{2}\right)}\right)\right]\right. \\
& \left.+\tau_{1}^{a}\left[-\frac{\vec{B}_{5} \cdot \vec{k}}{\left(q_{1}^{2}+M_{\pi}^{2}\right)^{2}\left(k^{2}+M_{\pi}^{2}\right)}+\frac{1}{q_{1}^{2}+M_{\pi}^{2}}\left(\frac{\vec{B}_{6} \cdot \vec{k}}{k^{2}\left(k^{2}+M_{\pi}^{2}\right)^{2}}+\frac{\vec{B}_{7} \cdot \vec{k}}{k^{2}\left(k^{2}+M_{\pi}^{2}\right)}\right)\right]\right\}+1 \leftrightarrow 2, \quad(8.39)
\end{aligned}
$$

where the vector quantities $\vec{B}_{i}$ are defined in Eq. (5.20). Similarly to the previously considered contributions, one notices that the pseudoscalar current $P_{2 \mathrm{~N}: 1 \pi}^{a\left(Q^{0}\right)}$ is directly related to the pion-pole terms in the corresponding axial current operator $\vec{A}_{2 \mathrm{~N}: 1 \pi, 1 / m}^{a(Q)}$ in Eq. (5.19). 
Last but not least, there are also contributions coming from time-derivatives of unitary transformations in diagrams (3) and (4) of Fig. 10 which are proportional to $k_{0}$, see Eq. (8.30). For the standard choice of the unitary phases, these are given by

$$
P_{2 \mathrm{~N}: 1 \pi, \mathrm{UT}^{\prime}}^{a\left(Q^{0}\right)}=i \frac{M_{\pi}^{2}}{m_{q} k^{2}} \vec{k} \cdot \vec{A}_{2 \mathrm{~N}: 1 \pi, \mathrm{UT}^{\prime}}^{a(Q)}
$$

where $\vec{A}_{2 \mathrm{~N}: 1 \pi, \mathrm{UT}^{\prime}}^{a}(Q)$ is given in Eq. (5.23).

\section{Two-pion-exchange contributions}

We now turn to the two-pion exchange contributions emerging from diagrams $(4-6),(10-12),(17),(18),(20)$ of Fig. 16. We found that graphs $(4-6)$ depend on the unitary phases via

$$
\begin{aligned}
(2),(3) & \sim 1+2 \alpha_{1}^{a x} \\
(5) & \sim-3+2 \alpha_{25}^{p}+2 \alpha_{26}^{p}, \\
(4),(6) & \sim\left(-2+\alpha_{4}^{p}-\alpha_{5}^{p}-\alpha_{6}^{p}+\alpha_{10}^{p}+\alpha_{13}^{p}\right) \frac{1}{\left(k^{2}+M_{\pi}^{2}\right)^{2}}[\ldots]+\left(-1+\alpha_{12}^{p}+\alpha_{15}^{p}\right) \frac{1}{k^{2}+M_{\pi}^{2}} .
\end{aligned}
$$

Notice that the contributions involving second-order pion-pole terms resulting from diagrams (4), (6) vanish for our standard choice of the unitary phases. The resulting pseudoscalar current can be expressed in terms of pion-pole contributions to the axial current operator $\vec{A}_{2 \mathrm{~N}: 2 \pi}^{a(Q)}$ given in Eq. (5.29) via

$$
P_{2 \mathrm{~N}: 2 \pi}^{a\left(Q^{0}\right)}=\left.i \frac{M_{\pi}^{2}}{m_{q} k^{2}} \vec{k} \cdot \vec{A}_{2 \mathrm{~N}: 2 \pi}^{a(Q)}\right|_{g_{13}=g_{14}=g_{15}=g_{16}=g_{17}=0},
$$

with the scalar functions $g_{1}\left(q_{1}\right), \ldots, g_{12}\left(q_{1}\right)$ being defined in Eq. (5.31).

\section{Short-range contributions to the pseudoscalar current at order $Q^{0}$}

We first consider the static contributions and begin with tree-level diagrams, which could emerge from the derivativeless terms in the effective Lagrangian $\mathcal{L}_{N N}$ involving a single insertion of the pseudoscalar source. It is, however, not possible to build such structures in the Lagrangian which fulfill all the required symmetries. Thus, there are no static tree-level contributions to the pseudoscalar current at this order.

Next, one-loop diagrams $(3-5),(8),(9),(17),(18),(25-30)$ of Fig. 17 involving a single insertion of the leading contact interactions from $\mathcal{L}_{N N}^{(0)}$ are found to yield nonvanishing contributions to the pseudoscalar current. The ones emerging from graphs $(3-5),(25)$ and $(27-30)$ depend on the unitary phases via

$$
\begin{aligned}
(3) & \sim\left(-2+\alpha_{4}^{p}-\alpha_{5}^{p}-\alpha_{6}^{p}+\alpha_{10}^{p}+\alpha_{13}^{p}\right) \frac{1}{\left(k^{2}+M_{\pi}^{2}\right)^{2}}[\ldots]+\left(-1+\alpha_{12}^{p}+\alpha_{15}^{p}\right) \frac{1}{k^{2}+M_{\pi}^{2}}[\ldots], \\
(4) & \sim\left(-4+\alpha_{4}^{p}-\alpha_{5}^{p}-\alpha_{6}^{p}+\alpha_{10}^{p}+\alpha_{13}^{p}+\alpha_{21}^{p}+\alpha_{22}^{p}+\alpha_{23}^{p}-\alpha_{24}^{p}\right) \frac{1}{\left(k^{2}+M_{\pi}^{2}\right)^{2}}[\ldots] \\
& +\left(-1+\alpha_{12}^{p}+\alpha_{15}^{p}\right) \frac{1}{k^{2}+M_{\pi}^{2}}[\ldots] \\
(5),(24),(28-30) & \sim-2+\alpha_{21}^{p}+\alpha_{22}^{p}+\alpha_{23}^{p}-\alpha_{24}^{p}, \\
(21) & \sim 1+\alpha_{1}^{a x}, \\
(23) & \sim \alpha_{1}^{a x}, \\
(25) & \sim\left(-2+\alpha_{4}^{p}-\alpha_{5}^{p}-\alpha_{6}^{p}+\alpha_{10}^{p}+\alpha_{13}^{p}\right) \frac{1}{\left(k^{2}+M_{\pi}^{2}\right)^{2}}[\ldots]+\left(-2+\alpha_{12}^{p}+\alpha_{15}^{p}\right) \frac{1}{k^{2}+M_{\pi}^{2}}[\ldots]
\end{aligned}
$$




$$
\begin{aligned}
(27) & \sim\left(-4+\alpha_{4}^{p}-\alpha_{5}^{p}-\alpha_{6}^{p}+\alpha_{10}^{p}+\alpha_{13}^{p}+\alpha_{21}^{a p}+\alpha_{22}^{p}+\alpha_{23}^{p}-\alpha_{24}^{p}\right) \frac{1}{\left(k^{2}+M_{\pi}^{2}\right)^{2}}[\ldots] \\
& +\left(\alpha_{12}^{p}+\alpha_{15}^{p}\right) \frac{1}{k^{2}+M_{\pi}^{2}}[\ldots] .
\end{aligned}
$$

For our standard choice, the total contribution of these diagrams is found to vanish. Given that the static onepion-contact terms in the $3 \mathrm{NF}$ at $\mathrm{N}^{3} \mathrm{LO}$ vanish after antisymmetrization, our result is consistent with the matching condition. Thus we conclude that there are no static contributions to the short-range pseudoscalar current operator at order $Q^{0}$.

In addition to the static terms, one also encounters relativistic $1 / m$-corrections involving a single insertion of the LO contact interactions from $\mathcal{L}_{N N}^{(0)}$ as shown in Fig. 18. The unitary phase dependence of the corresponding contributions is given by

$$
\begin{aligned}
(1) & \sim 1+\alpha_{16}^{p, 1 / m} \\
(2),(4),(5) & \sim \alpha_{21}^{p}+\alpha_{22}^{p}+\alpha_{23}^{p}-\alpha_{24}^{p}-2 \\
(3) & \sim \alpha_{17}^{p}+\alpha_{18}^{p}+\alpha_{19}^{p}-\alpha_{20}^{p}+\alpha_{21}^{p}+\alpha_{22}^{p}+\alpha_{23}^{p}-\alpha_{24}^{p}-4 \\
(6) & \sim \alpha_{17}^{p}+\alpha_{18}^{p}+\alpha_{19}^{p}-\alpha_{20}^{p}-2 .
\end{aligned}
$$

For our standard choice, the pseudoscalar current is expressed in terms of the axial current $\vec{A}_{2 \mathrm{~N}: \text { cont, } 1 / m}^{a(Q)}$ defined in Eq. (5.41) via

$$
P_{2 \mathrm{~N}: \mathrm{cont}, 1 / m}^{a\left(Q^{0}\right)}=i \frac{M_{\pi}^{2}}{m_{q} k^{2}} \vec{k} \cdot \vec{A}_{2 \mathrm{~N}: \text { cont }, 1 / m}^{a(Q)}
$$

Finally there are also contributions from diagram (5) of Fig. 10 which are proportional to $k^{0}$ and for this reason are demoted to order $Q^{0}$. For the standard choice of unitary phases, we find the contribution

$$
P_{2 \mathrm{~N}: \text { cont }, \mathrm{UT}^{\prime}}^{a\left(Q^{0}\right)}=i \frac{M_{\pi}^{2}}{m_{q} k^{2}} \vec{k} \cdot \vec{A}_{2 \mathrm{~N}: \mathrm{cont}, \mathrm{UT}^{\prime}}^{a(Q)},
$$

where $\vec{A}_{2 \mathrm{~N} \text { : cont, } \mathrm{UT}^{\prime}}^{a(Q)}$ is specified in Eq. $(5.43)$.

\section{Three-nucleon pseudoscalar operators}

We now discuss $3 \mathrm{~N}$ contributions to the pseudoscalar current operator which start to contribute at the same order $Q^{0}$ and emerge from tree-level diagrams constructed solely from the lowest-order vertices. 3N diagrams which yield non-vanishing contributions and which do not involve contact interactions are $(8-18)$ and $(22-26)$ of Fig. 20. Out of these diagrams, graphs $(8-18)$ yield expressions which depend on the unitary phases as follows:

$$
\begin{aligned}
(12),(13) & \sim-1+\alpha_{25}^{p}+\alpha_{26}^{p} \\
(17),(18) & \sim-2+\alpha_{25}^{p}+\alpha_{26}^{p}, \\
(8-11) & \sim\left(-2+\alpha_{4}^{p}-\alpha_{5}^{p}-\alpha_{6}^{p}+\alpha_{10}^{p}+\alpha_{13}^{p}\right) \frac{1}{\left(k^{2}+M_{\pi}^{2}\right)^{2}}[\ldots]+\left(-2+\alpha_{12}^{p}+\alpha_{15}^{p}\right) \frac{1}{k^{2}+M_{\pi}^{2}}[\ldots] \\
(14-16) & \sim\left(-2+\alpha_{4}^{p}-\alpha_{5}^{p}-\alpha_{6}^{p}+\alpha_{10}^{p}+\alpha_{13}^{p}\right) \frac{1}{\left(k^{2}+M_{\pi}^{2}\right)^{2}}[\ldots]+\left(\alpha_{12}^{p}+\alpha_{15}^{p}\right) \frac{1}{k^{2}+M_{\pi}^{2}}[\ldots] .
\end{aligned}
$$

For our standard choice of the unitary phases, the contributions from diagrams $(12-16)$ as well as all expressions involving second-order pion-pole terms turn out to vanish. In order to facilitate a comparison with the four-nucleon force at $\mathrm{N}^{3} \mathrm{LO}$, we write the resulting expression for the $3 \mathrm{~N}$ pseudoscalar current $P_{3 \mathrm{~N}: \pi}^{a\left(Q^{0}\right)}$ in the form

$$
P_{3 \mathrm{~N}: \pi}^{a\left(Q^{0}\right)}=-i \frac{2 F_{\pi}^{2} M_{\pi}^{2}}{g_{A} m_{q}} \sum_{i=5}^{8} \frac{\vec{C}_{i}^{a} \cdot \vec{k}}{k^{2}}+5 \text { permutations }=\left.i \frac{M_{\pi}^{2}}{m_{q} k^{2}} \vec{k} \cdot \vec{A}_{3 \mathrm{~N}: \pi}^{a(Q)}\right|_{\text {pion-pole terms }},
$$


where $\vec{C}_{i}^{a}$ are defined in Eq. (6.3).

Finally, diagrams $(5-19)$ of Fig. 21 involving one or more insertions of the the leading $2 \mathrm{~N}$ contact interactions also contribute to the $3 \mathrm{~N}$ pseudoscalar current. All these graphs yield expressions which depend upon the unitary phases:

$$
\begin{aligned}
(7),(9),(10),(13-19) & \sim-2+\alpha_{21}^{p}+\alpha_{22}^{p}+\alpha_{23}^{p}-\alpha_{24}^{p}, \\
(5),(6) & \sim\left(-2+\alpha_{4}^{p}-\alpha_{5}^{p}-\alpha_{6}^{p}+\alpha_{10}^{p}+\alpha_{13}^{p}\right) \frac{1}{\left(k^{2}+M_{\pi}^{2}\right)^{2}}[\ldots]+\left(-2+\alpha_{12}^{p}+\alpha_{15}^{p}\right) \frac{1}{k^{2}+M_{\pi}^{2}}[\ldots], \\
(8) & \sim\left(-2+\alpha_{4}^{p}-\alpha_{5}^{p}-\alpha_{6}^{p}+\alpha_{10}^{p}+\alpha_{13}^{p}\right) \frac{1}{\left(k^{2}+M_{\pi}^{2}\right)^{2}}[\ldots]+\left(\alpha_{12}^{p}+\alpha_{15}^{p}\right) \frac{1}{k^{2}+M_{\pi}^{2}}[\ldots], \\
(12) & \sim-2+\alpha_{25}^{p}+\alpha_{26}^{p}, \\
(11) & \sim\left(-4+\alpha_{4}^{p}-\alpha_{5}^{p}-\alpha_{6}^{p}+\alpha_{10}^{p}+\alpha_{13}^{p}+\alpha_{21}^{p}+\alpha_{22}^{p}+\alpha_{23}^{p}-\alpha_{24}^{p}\right) \frac{1}{\left(k^{2}+M_{\pi}^{2}\right)^{2}}[\ldots] \\
& +\left(\alpha_{12}^{p}+\alpha_{15}^{p}\right) \frac{1}{k^{2}+M_{\pi}^{2}}[\ldots] .
\end{aligned}
$$

For our standard choice, the contributions of graphs (7-11), (13-19) and all expressions involving second-order pionpole terms vanish yielding the result:

$$
P_{3 \mathrm{~N}: \text { cont }}^{a(Q)}=-i \frac{2 F_{\pi}^{2} M_{\pi}^{2}}{g_{A} m_{q}} \sum_{i=2}^{3} \frac{\vec{D}_{i}^{a} \cdot \vec{k}}{k^{2}}+5 \text { permutations }=\left.i \frac{M_{\pi}^{2}}{m_{q} k^{2}} \vec{k} \cdot \vec{A}_{3 \mathrm{~N}: \text { cont }}^{a(Q)}\right|_{\text {pion-pole terms }},
$$

with $\vec{D}_{i}^{a}$ defined in Eq. (6.7).

\section{SUMMARY OF THE DERIVED PSEUDOSCALAR CONTRIBUTIONS}

We now summarize the derived contributions to the nuclear pseudoscalar current operator

$$
\boldsymbol{P}=\boldsymbol{P}_{1 \mathrm{~N}}+\boldsymbol{P}_{2 \mathrm{~N}}+\boldsymbol{P}_{3 \mathrm{~N}}+\ldots
$$

The chiral power counting implies that $n$-nucleon operators are, in general, suppressed by two powers of the expansion parameter relative to $n-1$-nucleon operators so the one expects the hierarchy ${ }^{9}$

$$
\boldsymbol{P}_{1 \mathrm{~N}} \sim \mathcal{O}\left(Q^{-4}\right), \quad \boldsymbol{P}_{2 \mathrm{~N}} \sim \mathcal{O}\left(Q^{-2}\right), \quad \boldsymbol{P}_{3 \mathrm{~N}} \sim \mathcal{O}\left(Q^{0}\right), \quad \ldots
$$

Thus, at fourth order in the chiral expansion relative to the dominant one-body contribution at order, it is necessary and sufficient to include single-, two- and three-nucleon operators.

In Table III, we summarize all derived contributions to the nuclear pseudoscalar current up to $\mathrm{N}^{3} \mathrm{LO}$ based on our counting scheme for the nucleon mass and the standard choice of the unitary phases. As it is already clear from the previous section, the chiral expansion of the pseudoscalar current closely resembles that of the axial-vector current operator, see Table I, with the corresponding contributions appearing one order lower. Finally, notice that isospin breaking corrections to the exchange pseudoscalar current operator start contributing at order $Q$ which is beyond the accuracy of our analysis.

\section{CURRENT CONSERVATION}

Current conservation leads to the relation $(2.42)$ between the axial current, charge, pseudoscalar density $\hat{\overrightarrow{A^{a}}}\left(\vec{k}, k_{0}\right)$, $\hat{A}^{0, a}\left(\vec{k}, k_{0}\right), \hat{P}^{a}\left(\vec{k}, k_{0}\right)$ and the contributions to the nuclear Hamiltonian

$$
\hat{H}=\hat{H}_{1 \mathrm{~N}}^{\left(Q^{0}\right)}+\hat{V}_{2 \mathrm{~N}: 1 \pi}^{\left(Q^{0}\right)}+\hat{V}_{2 \mathrm{~N}: \text { cont }}^{\left(Q^{0}\right)}+\mathcal{O}\left(Q^{2}\right),
$$

\footnotetext{
9 The leading contribution to the two-nucleon current operator at order $Q^{-2}$ turns out to vanish.
} 
TABLE III: Chiral expansion of the nuclear pseudoscalar operator up to $\mathrm{N}^{3} \mathrm{LO}$.

\begin{tabular}{|c|c|c|c|}
\hline order & single-nucleon & two-nucleon & three-nucleon \\
\hline $\mathrm{LO}\left(Q^{-4}\right)$ & $P_{1 \mathrm{~N}: \text { static }}^{a}$, Eq. $(8.11)$ & - & - \\
\hline $\operatorname{NLO}\left(Q^{-2}\right)$ & $P_{1 \mathrm{~N}: \text { static }}^{a}$, Eq. (8.13) & - & - \\
\hline $\mathrm{N}^{2} \mathrm{LO}\left(Q^{-1}\right)$ & - & $\begin{array}{r}P_{2 \mathrm{~N}: 1 \pi}^{a}, \text { Eq. }(8.32) \\
+P_{2 \mathrm{~N}: \text { cont }}^{a}, \\
\text { Eq. }(8.33)\end{array}$ & - \\
\hline $\mathrm{N}^{3} \mathrm{LO}\left(Q^{0}\right)$ & $\begin{aligned} P_{1 \mathrm{~N}: \mathrm{static}}^{a}, & \text { Eq. }(8.27) \\
+P_{1 \mathrm{~N}: 1 / m, \mathrm{UT}^{\prime}}^{a}, & \text { Eq. }(8.16) \\
+P_{1 \mathrm{~N}: 1 / \mathrm{m}^{2}}^{a}, & \text { Eq. }(8.18)\end{aligned}$ & $\begin{aligned} P_{2 \mathrm{~N}: 1 \pi}^{a}, & \text { Eq. }(8.35) \\
+P_{2 \mathrm{~N}: 1 \pi, \mathrm{UT}^{\prime}}^{a}, & \text { Eq. }(8.40) \\
+P_{2 \mathrm{~N}: 1 \pi, 1 / m}^{a}, & \text { Eq. }(8.39) \\
+P_{2 \mathrm{~N}: 2 \pi}^{a}, & \text { Eq. }(8.42) \\
+P_{2 \mathrm{~N}: \text { cont, } \mathrm{UT}^{\prime}}^{a}, & \text { Eq. }(8.46) \\
+P_{2 \mathrm{~N}: \text { cont }, 1 / \mathrm{m}}^{a}, & \text { Eq. }(8.45)\end{aligned}$ & $\begin{aligned} P_{3 \mathrm{~N}: \pi}^{a}, & \text { Eq. }(8.48) \\
+P_{3 \mathrm{~N}: \mathrm{cont}}^{a}, & \text { Eq. }(8.50)\end{aligned}$ \\
\hline
\end{tabular}

where $\hat{X}$ means that the quantity $X$ is to be taken as an operator rather than matrix element with respect to the nucleon momenta, and $\hat{H}_{1 \mathrm{~N}}^{\left(Q^{0}\right)}$ refers to the nonrelativistic kinetic energy operator. Notice further that in order to get a correct chiral order for any sequence of operators in the convention we are using, one should take into account the suppression factor of $Q^{3}$ for every intermediate nucleonic state. For example, the chiral order of the operator $\hat{H}_{1 \mathrm{~N}}^{\left(Q^{0}\right)} \hat{A}_{1 \mathrm{~N}: 1 / m}^{0, a\left(Q^{-1}\right)}$ is $Q^{2}$, while that of $\hat{V}_{2 \mathrm{~N}: 1 \pi}^{\left(Q^{0}\right)} \hat{V}_{2 \mathrm{~N}: 1 \pi}^{\left(Q^{0}\right)} \hat{A}_{1 \mathrm{~N}: \mathrm{UT} \mathrm{T}^{\prime}}^{0, a\left(Q^{-1}\right)}$ is $Q^{5}$. Alternatively, one can, of course, also explicitly verify the chiral order of any sequence of operators by adding together the inverse mass dimension $\kappa$ of all vertices as explained in section II. Last but not least, we remind the reader that within the adopted counting scheme for the nucleon mass with $m \sim \Lambda_{b}^{2} / M_{\pi}$, the energy-transfer $k_{0}$ is counted as $k_{0} \sim Q^{2} / m=\mathcal{O}\left(Q^{3}\right)$.

In the following, we will explicitly verify the validity of the continuity equation for all derived contributions to the charge and current operators.

- Single-nucleon current operator

Requiring the continuity equation (2.42) to hold true at all considered orders in the chiral expansion, we obtain the relations

$$
\begin{aligned}
\vec{k} \cdot \hat{\vec{A}}_{1 \mathrm{~N}: \text { static }}^{a\left(Q^{-3}\right)}-m_{q} i \hat{P}_{1 \mathrm{~N}: \text { static }}^{a\left(Q^{-4}\right)}= & 0 \\
\vec{k} \cdot \hat{\vec{A}}_{1 \mathrm{~N}: \text { static }}^{a\left(Q^{-1}\right)}-m_{q} i \hat{P}_{1 \mathrm{~N}: \text { static }}^{a\left(Q^{-2}\right)}= & 0 \\
\vec{k} \cdot \hat{\vec{A}}_{1 \mathrm{~N}: 1 / m^{2}}^{a(Q)}-m_{q} i \hat{P}_{1 \mathrm{~N}: 1 / m^{2}}^{a\left(Q^{0}\right)} & =\left[\hat{H}_{1 \mathrm{~N}}^{\left(Q^{0}\right)}, \hat{A}_{1 \mathrm{~N}: 1 / m}^{0, a\left(Q^{-1}\right)}-\frac{\partial}{\partial k_{0}} \vec{k} \cdot \hat{\vec{A}}_{1 \mathrm{~N}: 1 / m, \mathrm{UT}^{\prime}}^{a(Q)}+\left[\hat{H}_{1 \mathrm{~N}}^{\left(Q^{0}\right)}, \frac{\partial}{\partial k_{0}} \hat{A}_{1 \mathrm{~N}: \mathrm{UT}^{\prime}}^{0, a}\right]\right. \\
& \left.+m_{q} i \frac{\partial}{\partial k_{0}} \hat{P}_{1 \mathrm{~N}: 1 / m, \mathrm{UT}}^{a\left(Q^{\prime}\right)}\right] \\
\vec{k} \cdot \hat{\vec{A}}_{1 \mathrm{~N}: \text { static }}^{a(Q)}-m_{q} i \hat{P}_{1 \mathrm{~N}: \text { static }}^{a\left(Q^{0}\right)} & =0 .
\end{aligned}
$$

It is easy to verify that the derived contributions fulfill the first three equations. Notice further that the last equation implies the Goldberger-Treiman-like relation between the LECs $f_{1}^{A}$ and $f_{1}^{P}$ :

$$
f_{1}^{P}=-4 f_{1}^{A} .
$$

It is then straightforward to verify the validity of the last relation in Eq. (10.5) using Eqs. (4.46) and (8.27).

- Two-nucleon current operator

At leading order, the continuity equation (2.42) leads to the relations

$$
\vec{k} \cdot \hat{\vec{A}}_{2 \mathrm{~N}: 1 \pi}^{a\left(Q^{0}\right)}-m_{q} i \hat{P}_{2 \mathrm{~N}: 1 \pi}^{a\left(Q^{-1}\right)}=0
$$




$$
\vec{k} \cdot \hat{\vec{A}}_{2 \mathrm{~N}: \text { cont }}^{a\left(Q^{0}\right)}-m_{q} i \hat{P}_{2 \mathrm{~N}: \text { cont }}^{a\left(Q^{-1}\right)}=0
$$

which are trivially fulfilled, see Eqs. (8.32) and (8.33).

At order $Q^{2}$, the continuity equation induces a number or relations between the various contributions. Identifying all order- $Q^{2}$ terms of the one-pion range in Eq. (2.42), we obtain the relation

$$
\vec{k} \cdot \hat{\vec{A}}_{2 \mathrm{~N}: 1 \pi}^{a(Q)}-m_{q} i \hat{P}_{2 \mathrm{~N}: 1 \pi}^{a\left(Q^{0}\right)}=\text { shorter-range terms . }
$$

Here, we used the fact that there are no static contributions to the single-nucleon charge operator for $k_{0}=0$ up to order $Q^{0}$. For this reason, there is no commutator of the one-pion-exchange potential with the single-nucleon charge operator at the considered order. Further, there are no static $k_{0}$-dependent contributions to the singlenucleon axial and pseudoscalar currents at orders $Q$ and $Q^{0}$, respectively. For this reason, there are no terms of the one-pion range on the left-hand side of Eq. (2.42) involving a single commutator at order $Q^{2}$. Finally, the double-commutator term involving a $k_{0}$-derivative of the single nucleon charge operator cannot give rise to operators of the one-pion range in the static limit. Notice further that the shorter-range terms on the right-hand side of Eq. (10.10) reflect the ambiguity in separating the one- and two-pion exchange contributions. Using the derived expressions for $\hat{\vec{A}}_{2 \mathrm{~N}: 1 \pi}^{a(Q)}$ and $\hat{P}_{2 \mathrm{~N}: 1 \pi}^{a\left(Q^{0}\right)}$ given in Eqs. (5.13) and (8.35), respectively, we obtain

$$
\vec{k} \cdot \hat{\vec{A}}_{2 \mathrm{~N}: 1 \pi}^{a(Q)}-m_{q} i \hat{P}_{2 \mathrm{~N}: 1 \pi}^{a\left(Q^{0}\right)}=\frac{g_{A}^{3}}{32 \pi F_{\pi}^{4}} \tau_{2}^{a} \vec{q}_{2} \cdot \vec{\sigma}_{2}\left(\left(1+g_{A}^{2}\right) M_{\pi}+\left(2 M_{\pi}^{2}+q_{1}^{2}\right) A\left(q_{1}\right)\right)+1 \leftrightarrow 2 .
$$

As expected, the expression on the right-hand side of Eq. (10.10) has no pole at $q_{1}^{2}=-M_{\pi}^{2}$ and can be cast into the form of a spectral integral taken over the region $\mu \geq 2 M_{\pi}$. It is important to keep these terms in mind when verifying the continuity equation for the two-pion exchange contributions. More precisely, the sum of the static one- and two-pion exchange contributions to the current operator, being unaffected by the above mentioned ambiguity in separating the terms according to the range, is expected to fulfill the relation

$$
\begin{aligned}
\vec{k} \cdot \hat{\vec{A}}_{2 \mathrm{~N}: 1 \pi}^{a(Q)}-m_{q} i \hat{P}_{2 \mathrm{~N}: 1 \pi}^{a\left(Q^{0}\right)}+\vec{k} \cdot \hat{\vec{A}}_{2 \mathrm{~N}: 2 \pi}^{a(Q)}-m_{q} i \hat{P}_{2 \mathrm{~N}: 2 \pi}^{a\left(Q^{0}\right)} & =\left[\hat{V}_{2 \mathrm{~N}: 1 \pi}^{\left(Q^{0}\right)}, \hat{A}_{2 \mathrm{~N}: 1 \pi}^{0, a\left(Q^{-1}\right)}-\frac{\partial}{\partial k_{0}} \vec{k} \cdot \hat{\vec{A}}_{2 \mathrm{~N}: 1 \pi, \mathrm{UT}^{\prime}}^{a(Q)}\right. \\
& \left.+\frac{\partial}{\partial k_{0}}\left[\hat{V}_{2 \mathrm{~N}: 1 \pi}^{\left(Q^{0}\right)}, \hat{A}_{1 \mathrm{~N}: \mathrm{UT}^{\prime}}^{0, a\left(Q^{-1}\right)}\right]+m_{q} i \frac{\partial}{\partial k_{0}} \hat{P}_{2 \mathrm{~N}: 1 \pi, \mathrm{UT}^{\prime}}^{a\left(Q^{0}\right)}\right]
\end{aligned}
$$

Using the derived expressions for the various charge and current operators and applying dimensional regularization to evaluate the integrals appearing on the right-hand side, we have explicitly verified that this equation indeed holds true.

Consider now static contributions to the continuity equation at order $Q^{2}$, which involve contact interactions. Since we have only vanishing terms in both the short-range axial and pseudoscalar current operators at orders $Q$ and $Q^{2}$, respectively, the right-hand side of Eq. (2.42) vanishes trivially. On the other hand, the left-hand side of this equation does contain non-vanishing terms. Notice, however, that since there is no static singlenucleon charge operator at order $Q^{-1}$ for $k_{0}=0$, we do not have any commutator with it. Similarly, we have $\hat{A}_{2 \mathrm{~N} \text { : cont }}^{0, a\left(Q^{-1}\right)}=0$ so that there cannot be any commutator with this operator. Furthermore, due to the absence of static $k_{0}$-dependent contributions to the single-nucleon pseudoscalar current up to order $Q^{-1}$, there is also no commutator of this operator with the effective Hamiltonian. Collecting the remaining static order- $Q^{2}$ short-range contributions on the left-hand side of Eq. (2.42), we end up with the relation

$$
\begin{aligned}
0 & =\left[\hat{V}_{2 \mathrm{~N}: \mathrm{cont}}^{\left(Q^{0}\right)}, \hat{A}_{2 \mathrm{~N}: 1 \pi}^{0, a\left(Q^{-1}\right)}-\frac{\partial}{\partial k_{0}} \vec{k} \cdot \hat{\vec{A}}_{2 \mathrm{~N}: 1 \pi, \mathrm{UT}^{\prime}}^{a(Q)}+\frac{\partial}{\partial k_{0}}\left[\hat{V}_{2 \mathrm{~N}: 1 \pi}^{\left(Q^{0}\right)}, \hat{A}_{1 \mathrm{~N}: \mathrm{UT}}^{0, a\left(Q^{-1}\right)}\right]+m_{q} i \frac{\partial}{\partial k_{0}} \hat{P}_{2 \mathrm{~N}: 1 \pi, \mathrm{UT}^{\prime}}^{a\left(Q^{0}\right)}\right] \\
& +\left[\hat{V}_{2 \mathrm{~N}: 1 \pi}^{\left(Q^{0}\right)},-\frac{\partial}{\partial k_{0}} \vec{k} \cdot \hat{\vec{A}}_{2 \mathrm{~N}: \mathrm{cont}, \mathrm{UT}^{\prime}}^{a(Q)}+\frac{\partial}{\partial k_{0}}\left[\hat{V}_{2 \mathrm{~N}: \mathrm{cont}}^{\left(Q^{0}\right)}, \hat{A}_{1 \mathrm{~N}: \mathrm{UT}^{\prime}}^{0, a\left(Q^{-1}\right)}\right]+m_{q} i \frac{\partial}{\partial k_{0}} \hat{P}_{2 \mathrm{~N}: \mathrm{cont}, \mathrm{UT}^{\prime}}^{a\left(Q^{0}\right)}\right] .
\end{aligned}
$$

Using the derived contributions to the current operators, we have verified that this relation is indeed fulfilled. In fact, we even find

$$
0=-\frac{\partial}{\partial k_{0}} \vec{k} \cdot \hat{\vec{A}}_{2 \mathrm{~N}: \mathrm{cont}, \mathrm{UT}^{\prime}}^{a(Q)}+\frac{\partial}{\partial k_{0}}\left[\hat{V}_{2 \mathrm{~N}: \mathrm{cont}}^{\left(Q^{0}\right)}, \hat{A}_{1 \mathrm{~N}: \mathrm{UT}^{\prime}}^{0, a\left(Q^{-1}\right)}\right]+m_{q} i \frac{\partial}{\partial k_{0}} \hat{P}_{2 \mathrm{~N}: \mathrm{cont}, \mathrm{UT}^{\prime}}^{a\left(Q^{0}\right)}
$$

so that Eq. (10.12) simplifies to

$$
0=\left[\hat{V}_{2 \mathrm{~N}: \mathrm{cont}}^{\left(Q^{0}\right)}, \hat{A}_{2 \mathrm{~N}: 1 \pi}^{0, a\left(Q^{-1}\right)}-\frac{\partial}{\partial k_{0}} \vec{k} \cdot \hat{\vec{A}}_{2 \mathrm{~N}: 1 \pi, \mathrm{UT}^{\prime}}^{a(Q)}+\frac{\partial}{\partial k_{0}}\left[\hat{V}_{2 \mathrm{~N}: 1 \pi}^{\left(Q^{0}\right)}, \hat{A}_{1 \mathrm{~N}: \mathrm{UT}^{\prime}}^{0, a\left(Q^{-1}\right)}\right]+m_{q} i \frac{\partial}{\partial k_{0}} \hat{P}_{2 \mathrm{~N}: 1 \pi, \mathrm{UT}^{\prime}}^{a\left(Q^{0}\right)}\right] .
$$


Next, for the $1 / m$-corrections to one-pion-exchange two-nucleon axial-vector current, the continuity equation implies the relation

$$
\begin{aligned}
\vec{k} \cdot \hat{\vec{A}}_{2 \mathrm{~N}: 1 \pi, 1 / m}^{a(Q)}-m_{q} i \hat{P}_{2 \mathrm{~N}: 1 \pi, 1 / m}^{a\left(Q^{0}\right)} & =\left[\hat{H}_{1 \mathrm{~N}}^{\left(Q^{0}\right)}, \hat{A}_{2 \mathrm{~N}: 1 \pi}^{0, a\left(Q^{-1}\right)}-\frac{\partial}{\partial k_{0}} \vec{k} \cdot \hat{\vec{A}}_{2 \mathrm{~N}: 1 \pi, \mathrm{UT}^{\prime}}^{a(Q)}+\frac{\partial}{\partial k_{0}}\left[\hat{V}_{2 \mathrm{~N}: 1 \pi}^{\left(Q^{0}\right)}, \hat{A}_{1 \mathrm{~N}: \mathrm{UT}^{\prime}}^{0, a}\right]\right. \\
& \left.+m_{q} i \frac{\partial}{\partial k_{0}} \hat{P}_{2 \mathrm{~N}: 1 \pi, \mathrm{UT}^{\prime}}^{a\left(Q^{0}\right)}\right]+\left[\hat{V}_{2 \mathrm{~N}: 1 \pi}^{\left(Q^{0}\right)}, \hat{A}_{1 \mathrm{~N}: 1 / m}^{0, a\left(Q^{-1}\right)}-\frac{\partial}{\partial k_{0}} \vec{k} \cdot \hat{\vec{A}_{1 \mathrm{~N}: 1 / m, \mathrm{UT}^{\prime}}^{a(Q)}}\right. \\
& \left.+\frac{\partial}{\partial k_{0}}\left[\hat{H}_{1 \mathrm{~N}}^{\left(Q^{0}\right)}, \hat{A}_{1 \mathrm{~N}: \mathrm{UT}^{\prime}}^{0, a\left(Q^{-1}\right)}\right]+m_{q} i \frac{\partial}{\partial k_{0}} \hat{P}_{1 \mathrm{~N}: 1 / m, \mathrm{UT}^{\prime}}^{a\left(Q^{0}\right)}\right]
\end{aligned}
$$

which is indeed fulfilled for the derived operators. We have also explicitly verified the validity of the continuity equation for the $1 / m$-corrections to the $2 \mathrm{~N}$ axial vector current involving contact interactions:

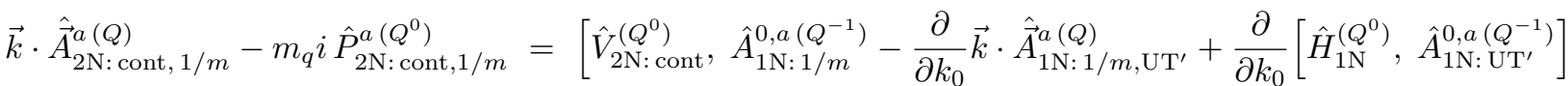

$$
\begin{aligned}
& \left.+m_{q} i \frac{\partial}{\partial k_{0}} \hat{P}_{1 \mathrm{~N}: 1 / m, \mathrm{UT}^{\prime}}^{a\left(Q^{0}\right)}\right]
\end{aligned}
$$

One may also expect further contributions to the right-hand side of Eq. (10.16) stemming from the commutator with the kinetic energy. However, as already pointed out, those contributions turn out to vanish, see Eq. (10.13).

- Three-nucleon current operator

Finally, we have verified that the following two relations are fulfilled for the derived three-nucleon current operator:

$$
\begin{aligned}
\vec{k} \cdot \hat{\vec{A}}_{3 \mathrm{~N}: \pi}^{a(Q)}-m_{q} i \hat{P}_{3 \mathrm{~N}: \pi}^{a\left(Q^{0}\right)} & =\left[\hat{V}_{2 \mathrm{~N}: 1 \pi}^{\left(Q^{0}\right)}, \hat{A}_{2 \mathrm{~N}: 1 \pi}^{0, a\left(Q^{-1}\right)}-\frac{\partial}{\partial k_{0}} \vec{k} \cdot \hat{\vec{A}}_{2 \mathrm{~N}: 1 \pi, \mathrm{UT}^{\prime}}^{a(Q)}+\frac{\partial}{\partial k_{0}}\left[\hat{V}_{2 \mathrm{~N}: 1 \pi}^{\left(Q^{0}\right)}, \hat{A}_{1 \mathrm{~N}: \mathrm{UT}^{\prime}}^{0, a\left(Q^{-1}\right)}\right]\right. \\
& \left.+m_{q} i \frac{\partial}{\partial k_{0}} \hat{P}_{2 \mathrm{~N}: 1 \pi, \mathrm{UT}^{\prime}}^{a\left(Q^{0}\right)}\right] \\
\vec{k} \cdot \hat{\vec{A}}_{3 \mathrm{~N}: \text { cont }}^{a(Q)}-m_{q} i \hat{P}_{3 \mathrm{~N}: \mathrm{cont}}^{a\left(Q^{0}\right)} & =\left[\hat{V}_{2 \mathrm{~N}: \mathrm{cont}}^{\left(Q^{0}\right)}, \hat{A}_{2 \mathrm{~N}: 1 \pi}^{0, a\left(Q^{-1}\right)}-\frac{\partial}{\partial k_{0}} \vec{k} \cdot \hat{\vec{A}}_{2 \mathrm{~N}: 1 \pi, \mathrm{UT}^{\prime}}^{a(Q)}+\frac{\partial}{\partial k_{0}}\left[\hat{V}_{2 \mathrm{~N}: 1 \pi}^{\left(Q^{0}\right)}, \hat{A}_{1 \mathrm{~N}: \mathrm{UT}^{\prime}}^{0, a\left(Q^{-1}\right)}\right]\right. \\
& \left.+m_{q} i \frac{\partial}{\partial k_{0}} \hat{P}_{2 \mathrm{~N}: 1 \pi, \mathrm{UT}^{\prime}}^{a\left(Q^{0}\right)}\right] .
\end{aligned}
$$

Again, we made use of Eq. (10.13) to simplify the right-hand side of Eq. (10.18).

To summarize, we have explicitly verified the validity of the continuity equation for all derived contributions to the current operators.

\section{COMPARISON WITH EARLIER WORK}

We are now in the position to compare our results with the earlier derivations and begin with the single-nucleon contributions. To the best of our knowledge, the most complete expressions for the single-nucleon axial charge and current operators up-to-and-including $1 / \mathrm{m}^{2}$-corrections are given in Ref. [75] in terms of the axial and pseudoscalar form factors of the nucleon. Our results for the single-nucleon charge and current operators agree with the ones of that work up-to-and-including $1 / m$-terms. More precisely, our expression for the charge operator in the first line of Eq. (4.47) agrees with the expressions in Eq. (3.8) and (3.14) of Ref. [75]. Also the static contributions to the current operator given by the first two terms in the right-hand side of the second equation in (4.47) coincide with the corresponding terms in Eqs. (3.9) and (3.14) of that work. On the other hand, for a general choice of the unitary phases $\bar{\beta}_{8,9}$, we find non-vanishing contributions to the current operator $\vec{A}_{1 \mathrm{~N}: 1 / m, \mathrm{UT}^{\prime}}^{a(Q)}$ proportional to $k_{0} / m$, see Eq. (4.13). The absence of such contributions in Ref. [75] is consistent with the choice of $\bar{\beta}_{8}=\bar{\beta}_{9}=-1 / 2$. On the other hand, our results for $1 / m^{2}$-contributions $\vec{A}_{1 \mathrm{~N}: 1 / \mathrm{m}^{2}}^{a}$ in Eq. (4.18) appear to differ from the ones in Eq. (3.9) of Ref. [75] even for the choice $\bar{\beta}_{8}=\bar{\beta}_{9}=-1 / 2$. 
We now turn to the exchange axial current operators. As already pointed out, their first derivation within the framework of chiral EFT has been carried out by Park et al. [41, 42]. Given the incompleteness of this calculation, which did not include some of the reducible-like diagrams and ignored pion-pole contributions, we refrain from a detailed comparison with that work. A more complete recent derivation of the exchange axial currents has been carried out by Baroni et al. $[56,76]$ in the framework of TOPT. As already pointed out above, the dominant contributions to the two-nucleon charge and current operators $A_{2 \mathrm{~N}: 1 \pi}^{0, a\left(Q^{-1}\right)}$ and $\vec{A}_{2 \mathrm{~N}: 1 \pi}^{a\left(Q^{0}\right)}+\vec{A}_{2 \mathrm{~N}: \text { cont }}^{a\left(Q^{0}\right)}$ are well known, and our results for these terms agree with the ones derived using TOPT in Ref. [56]. The first corrections to the dominant two-nucleon terms emerge at order $Q$, see Tables I and II. While the authors of [56] count the nucleon mass in a different way as $m \sim \Lambda_{b}$, which implies that the relativistic corrections are promoted to lower orders in the chiral expansion as compared to our approach based on the assignment $m \sim \Lambda_{b}^{2} / M_{\pi}$, they have not considered the $1 / m$-contributions to the exchange operators and focused entirely on the static terms at order $Q .{ }^{10}$ They also do not give the contributions proportional to the energy transfer. For the remaining static contributions to the two-nucleon current operator $\vec{A}_{2 \mathrm{~N}: 1 \pi}^{a(Q)}+\vec{A}_{2 \mathrm{~N}: 2 \pi}^{a(Q)}$, our expressions differ from the ones given in Refs. [56, 76]. Given that the explicit expressions for these operators are rather involved, we restrict ourselves to the case of zero momentum transfer, $\vec{k}=0$, and compare the expressions for the sum of the one- and two-pion exchange operators at order $Q .{ }^{11}$ Notice that due to to a different counting of the nucleon mass, which results in the NLO contributions to $\boldsymbol{A}_{1 \mathrm{~N}}^{\mu}$ appearing already at order $Q^{-2}$ rather than at order $Q^{-1}$ as in our counting scheme, the authors of $[56,76]$ regard the order- $Q$ contributions as being $\mathrm{N}^{4} \mathrm{LO}$. The expressions for the corresponding one- and more-pion exchange (MPE) operators $\vec{j}_{a}^{\mathrm{N} 4 \mathrm{LO}}\left(\mathrm{MPE}, \vec{q}_{1}\right)$ and $\vec{j}_{a}^{\mathrm{N} 4 \mathrm{LO}}\left(\mathrm{MPE}, \vec{q}_{1}\right)$ are given in Eqs. (16) and (17) of Ref. [76]. Only the MPE part of their current operator depends on the loop functions

$$
\begin{aligned}
\vec{j}_{a}^{\mathrm{N} 4 L O}\left(\mathrm{MPE}, \vec{q}_{1}\right) & =\frac{g_{A}^{3}}{32 \pi F_{\pi}^{4}} \tau_{2}^{a}\left[W_{1}\left(q_{1}\right) \vec{\sigma}_{1}+W_{2}\left(q_{1}\right) \vec{q}_{1} \vec{\sigma}_{1} \cdot \vec{q}_{1}+Z_{1}\left(q_{1}\right)\left(2 \vec{q}_{1} \vec{\sigma}_{2} \cdot \vec{q}_{1} \frac{1}{q_{1}^{2}+M_{\pi}^{2}}-\vec{\sigma}_{2}\right)\right] \\
& +\frac{g_{A}^{5}}{32 \pi F_{\pi}^{4}} \tau_{1}^{a} W_{3}\left(q_{1}\right)\left(\vec{\sigma}_{2} \times \vec{q}_{1}\right) \times \vec{q}_{1}-\frac{g_{A}^{3}}{32 \pi F_{\pi}^{4}}\left[\boldsymbol{\tau}_{1} \times \boldsymbol{\tau}_{2}\right]^{a} Z_{3}\left(q_{1}\right) \vec{\sigma}_{1} \times \vec{q}_{1} \vec{\sigma}_{2} \cdot \vec{q}_{1} \frac{1}{q_{1}^{2}+M_{\pi}^{2}} \\
& +1 \leftrightarrow 2,
\end{aligned}
$$

where the loop functions in our notation are given by

$$
\begin{aligned}
W_{1}\left(q_{1}\right) & =\frac{1}{2} A\left(q_{1}\right)\left[4\left(1-2 g_{A}^{2}\right) M_{\pi}^{2}+\left(1-5 g_{A}^{2}\right) q_{1}^{2}\right]+\frac{1}{2} M_{\pi}\left[g_{A}^{2}\left(\frac{4 M_{\pi}^{2}}{4 M_{\pi}^{2}+q_{1}^{2}}-9\right)+1\right], \\
W_{2}\left(q_{1}\right) & =\frac{M_{\pi}\left(4\left(2 g_{A}^{2}+1\right) M_{\pi}^{2}+\left(3 g_{A}^{2}+1\right) q_{1}^{2}\right)}{2 q_{1}^{2}\left(4 M_{\pi}^{2}+q_{1}^{2}\right)}-\frac{A\left(q_{1}\right)\left(4\left(2 g_{A}^{2}+1\right) M_{\pi}^{2}+\left(g_{A}^{2}-1\right) q_{1}^{2}\right)}{2 q_{1}^{2}}, \\
W_{3}\left(q_{1}\right) & =-\frac{4 A\left(q_{1}\right)}{3}-\frac{1}{6 M_{\pi}}, \\
Z_{1}\left(q_{1}\right) & =2 A\left(q_{1}\right)\left(2 M_{\pi}^{2}+q_{1}^{2}\right)+2 M_{\pi}, \\
Z_{3}\left(q_{1}\right) & =\frac{1}{2} A\left(q_{1}\right)\left(4 M_{\pi}^{2}+q_{1}^{2}\right)+\frac{M_{\pi}}{2} .
\end{aligned}
$$

In strong contrast with our results, we note that due to the appearance of the function $W_{3}\left(q_{1}\right)$, the current operator of Baroni et al. does not exist in the chiral limit. Even relaxing all matching and renormalizability constraints on the unitary phases and requiring only the factorization constraints of Eq. (2.117), we are unable to find a choice of unitary phases which would bring our results in agreement with the ones of Baroni et al.. ${ }^{12}$ In the restrictive kinematics with $\vec{k}=0$, the dependence of only one unitary phase, namely $\alpha_{1}^{a x}$, survives for the axial-vector currents. Subtracting our result from the one of Baroni et al., we obtain for the difference

$$
\vec{j}_{a}^{\mathrm{N} 4 \mathrm{LO}}\left(\mathrm{MPE}, \vec{q}_{1}\right)-\vec{A}_{2 \mathrm{~N}: 2 \pi}^{a(Q)}-\vec{A}_{2 \mathrm{~N}: 1 \pi}^{a(Q)}=A\left(q_{1}\right)\left(\frac { \alpha _ { 1 } ^ { \mathrm { ax } } g _ { A } ^ { 5 } } { 2 5 6 \pi F _ { \pi } ^ { 4 } q _ { 1 } ^ { 2 } } \left[4 q_{1}^{2}\left[\boldsymbol{\tau}_{1} \times \boldsymbol{\tau}_{2}\right]^{a}\left(-\vec{q}_{1} \vec{q}_{1} \cdot \vec{\sigma}_{1} \times \vec{\sigma}_{2}-\vec{q}_{1} \times \vec{\sigma}_{2} \vec{q}_{1} \cdot \vec{\sigma}_{1}\right)\right.\right.
$$

\footnotetext{
10 Notice that in order to define the static two-pion exchange current operator via matching to the $S$-matrix, one needs to specify the $1 / m$-corrections to the one-pion exchange current. Given that the authors of [56] have neglected the non-static corrections to the energy denominators when calculating time-ordered diagrams, it is not clear to us that their result for the static two-pion exchange contributions is complete.

11 The separation into one- and two-pion exchange terms is ambiguous.

12 The constraints of Eq. (2.117) have to be imposed since the results Baroni et al. are given in a factorizable form.
} 


$$
\begin{aligned}
& +\tau_{1}^{a}\left(-2 \vec{q}_{1}\left(8 M_{\pi}^{2}-q_{1}^{2}\right) \vec{q}_{1} \cdot \vec{\sigma}_{2}+\left(8 M_{\pi}^{2}+q_{1}^{2}\right)\left(2 q_{1}^{2} \vec{\sigma}_{2}+\vec{q}_{1} \vec{q}_{1} \cdot \vec{\sigma}_{1}\right)\right. \\
& \left.\left.\left.-\vec{\sigma}_{1}\left(24 M_{\pi}^{2} q_{1}^{2}+11 q_{1}^{4}\right)\right)\right]-\frac{g_{A}^{5}\left(\vec{\sigma}_{2} \tau_{1}^{a} q_{1}^{4}+2 \vec{q}_{1}\left(6 M_{\pi}^{2}+q_{1}^{2}\right) \vec{q}_{1} \cdot \vec{\sigma}_{2} \tau_{1}^{a}\right)}{96 \pi F_{\pi}^{4} q_{1}^{2}}\right) \\
& + \text { rational function of } \vec{q}_{1}+1 \leftrightarrow 2 .
\end{aligned}
$$

As one can see from the above equation, we have to set $\alpha_{1}^{a x}=0$ in order to eliminate the term proportional to $\left[\boldsymbol{\tau}_{1} \times \boldsymbol{\tau}_{2}\right]^{a}$, which in turn is consistent with the renormalizability constraints. However, after setting $\alpha_{1}^{a x}=0$, we still obtain a difference:

$$
\begin{aligned}
\vec{j}_{a}^{\mathrm{N} 4 \mathrm{LO}}\left(\mathrm{MPE}, \vec{q}_{1}\right)-\vec{A}_{2 \mathrm{~N}: 2 \pi}^{a(Q)}-\left.\vec{A}_{2 \mathrm{~N}: 1 \pi}^{a(Q)}\right|_{\alpha_{1}^{a x}=0} & =-\frac{g_{A}^{5} A\left(q_{1}\right)\left(\vec{\sigma}_{2} \tau_{1}^{a} q_{1}^{4}+2 \vec{q}_{1}\left(6 M_{\pi}^{2}+q_{1}^{2}\right) \vec{q}_{1} \cdot \vec{\sigma}_{2} \tau_{1}^{a}\right)}{96 \pi F_{\pi}^{4} q_{1}^{2}} \\
& + \text { rational function of } \vec{q}_{1}+1 \leftrightarrow 2 .
\end{aligned}
$$

Thus, no choice of unitary phases makes our results agree with the ones of Baroni et al.. We conclude that our current operator and that of Baroni et al. are unitary non-equivalent (within the set of unitary transformations employed in our analysis). ${ }^{13}$ Since the loop function $A\left(q_{1}\right)$ affects the long-range behavior of the current, we get a disagreement even for the long-range terms.

Concerning the axial charge operator, our results for the two-pion exchange and short-range contributions $A_{2 \mathrm{~N}: 2 \pi}^{0, a}$ and $A_{2 \mathrm{~N} \text { : cont }}^{0, a}$ at order $Q$ agree with the ones of Ref. [56]. For the one-pion exchange contributions, we find the same expressions for the chiral logarithms, i.e. those terms in Eq. (5.14) which involve the loop function $L(q)$. This is not surprising given that they originate solely from the irreducible topologies (26) and (28) in Fig. 11 and thus can be obtained by evaluating the corresponding Feynman diagrams. On the other hand, the contributions to the scalar functions $h_{6,7,8}$ in Eq. (5.14), which are given by rational functions of momenta and the pion mass, differ from the corresponding terms found by Baroni and collaborators.

Finally, what concerns the three-nucleon axial current operator, whose leading terms emerge at order $Q$, Baroni et al. only consider in Ref. [76] the contributions of diagrams (21) and (25) in Fig. 20. To the best of our knowledge, our results in Eqs. (6.2) and (6.6) represent the first complete derivation of the dominant contributions to the three-nucleon axial current operator $\vec{A}_{3 \mathrm{~N}: \pi}^{a}+\vec{A}_{3 \mathrm{~N} \text { : cont }}^{a}$.

\section{SUMMARY AND CONCLUSIONS}

In this paper, we have analyzed in detail the nuclear axial-vector charge and current operators as well as the pseudoscalar currents in the framework of heavy-baryon chiral effective field theory. The main results of our study can be summarized below.

- First, we have worked out the general form of the continuity equation for the nuclear iso-triplet vector and axial-vector current operators based on the effective chiral Lagrangian involving (first-order) time derivatives of external sources. The resulting continuity equations (2.42) and (2.42) differ from their commonly assumed form by terms involving energy-transfer-dependent contributions to the charge and current operators.

- We have worked out Poincaré invariance constraints on the axial-vector charge and current operators which manifest themselves in the on-shell relation (2.77) between the effective Hamiltonian, boost and current operators. We have extended a formal proof of Ref. [64] that the generators of the Poincaré group acting in the Fock space of nucleons and mesons are simultaneously block diagonalized by the Okubo unitary transformation to the case of general interactions between the particles. This makes the proof valid for the effective operators derived in the framework of chiral EFT. We have also proposed an efficient way of calculating the effective boost operator which acts on the nucleonic part of the Fock space. Using this approach, we were able to explicitly verify Poincaré invariance constraints for the derived current operators.

${ }^{13}$ We can, however, not exclude the possibility of existence of a different set of unitary transformations, which would relate the two expressions for the current operator. 
- We have performed a complete derivation of the nuclear axial-vector charge and current operators to order $Q$ in the chiral expansion, i.e. to fourth order relative to the dominant one-body contribution, using the method of unitary transformation. The resulting currents are, per construction, consistent with the nuclear forces worked out in Refs. $[9,10,12-16,36]$ within the same approach. To render the loop integrals finite by the counterterms in the effective Lagrangian, we had to exploit the unitary ambiguity of the current operator in a systematic way. To this aim, we have considered a large class of unitary transformations on the nucleonic subspace of the Fock space, which are compatible with the chiral order we are working at and reduce to the identity operation when the external axial sources are switched off. The renormalized expressions for the current operators are found to feature a substantial degree of unitary ambiguity. We have argued that it is advantageous to choose the unitary phases, undetermined by the renormalizability constraint, in such a way that the pion-pole contributions to the axial current operator match the corresponding irreducible pion production amplitudes in the nuclear potentials. This particular choice of the unitary phases, which we refer to as standard, does not only appear natural, but is expected to simplify the regularization of the current operators in the way consistent with regularization of the nuclear forces. After matching to the nuclear forces, we end up with unambiguous expressions for the axial charge and current operators which are summarized in Tables I and II. To the best of our knowledge, the energy-transfer-dependent terms and relativistic corrections to the exchange operators have never been studied before in the framework of chiral EFT. Furthermore, for three-body operators, only irreducible-like topologies have been considered in the past, which, in fact, only constitute a small subset of the relevant diagrams.

- Using the same approach, we have independently derived the iso-triplet pseudoscalar current operator to fourth order relative to the dominant one-body contribution. After renormalization and matching to the nuclear forces, the expressions for the pseudoscalar currents do not show any unitary ambiguity. Our final results for the pseudoscalar current operator are summarized in Table III. To the best of our knowledge, the pseudoscalar nuclear current operators have never been studied before in the framework of chiral EFT.

- We have explicitly verified that the continuity equation (2.42) is valid for all derived contributions to the charge and current operators.

- We have compared our results for energy-transfer-independent static contributions to the two-body axial charge and current operators with the recent calculation by Baroni et al. [56, 76]. While our expressions for the static two-pion exchange and short-range contributions to the $2 \mathrm{~N}$ charge operator at order $Q$ agree with the ones of Ref. [56], there are differences in terms of the one-pion range. For the axial current operator, our static expressions differ strongly from the ones found by Baroni et al. We have illustrated this by considering a particular case of the threshold kinematics with $\vec{k}=0$. Notice that we have not succeeded to reproduce the expressions of Ref. [76] even by relaxing our constraints on the unitary phases. We further emphasize that in contrast to our results, the current operator derived by Baroni et al. does not exist in the chiral limit.

The results of our work provide a solid basis for theoretical investigations of weak processes in few- and many-nucleon systems. The derived expressions for the currents are consistent with the chiral $2 \mathrm{~N}$ potentials of Refs. $[5,21,77]^{14}$ and $3 \mathrm{~N}$ forces given in Refs. [12-15]. It would be interesting to test the novel current operators by calculating the triton $\beta$-decay and muon capture on ${ }^{3} \mathrm{He}$, for which precise experimental data are available. Work along these lines is in progress, see also Ref. [40, 76] for related recent studies. Notice further that the tritium half-life would come out as a parameter-free prediction up to $\mathrm{N}^{3} \mathrm{LO}$ once the LEC $D$, which governs the short-range behavior of both the axial current and three-nucleon force at $\mathrm{N}^{2} \mathrm{LO}$, is determined in the strong sector. It would also be interesting to analyze muon capture on the deuteron which is currently being measured by the MuSun experiment at PSI [53]. We also expect our findings to be relevant for a better understanding of the quenching of $g_{A}$ in nuclei, which is important for analyzing the ongoing and future experiments on neutrinoless double beta decay [78].

\section{Acknowledgments}

We would like to thank Vadim Baru, Arseniy Filin, Jacek Golak, Fred Myhrer and Roman Skibiński for sharing their insights into the discussed topics and useful comments on the manuscript. This work was supported by DFG

${ }^{14}$ To maintain consistency at the level of relativistic corrections, one will have to refit the $\mathrm{N}^{3} \mathrm{LO}$ and $\mathrm{N}^{4} \mathrm{LO}$ potentials of Refs. [5, 21] using the relativistic version of the Schrödinger equation (3.5) similar to what has been done in Ref. [77]. This work is in progress. 
(SFB/TR 110, "Symmetries and the Emergence of Structure in QCD") and BMBF (contract No. 05P2015 - NUSTAR R\&D). Further, this research was supported in part by the National Science Foundation under Grant No. NSF PHY1125915. The work of UGM was supported in part by The Chinese Academy of Sciences (CAS) President's International Fellowship Initiative (PIFI) grant no. 2015VMA076.

\section{Appendix A: Block diagonalization of the generators of the Poincaré group}

In this Appendix we will sketch the proof that Poincaré algebra gets simultaneously block diagonalized by the Okubo unitary transformation. The main steps are already described in [64]. However, in [64] only a special case of Yukawalike interactions has been considered. Here, we extend the proof to the case of arbitrary interactions. We will not repeat the proof of translational and rotational invariance of the operator $A$, which parametrizes the Okubo UT. These statements are independent of the form of the interactions and, for this reason, the proof can be taken from [64].

We begin with decomposing the boost and Hamiltonian operators into the free and interacting parts

$$
K^{j}=K_{0}^{j}+K_{I}^{j}, \quad H_{s}=H_{0}+H_{I} .
$$

From Poincaré algebra, one immediately obtains the relations

$$
\left[K_{0}^{j}, H_{0}\right]=i P^{j}, \quad\left[K_{I}^{j}, H_{0}\right]+\left[K_{0}^{j}, H_{I}\right]=0, \quad\left[K_{I}^{j}, H_{I}\right]=0, \quad\left[K_{0}^{j}, F\left(H_{0}\right)\right]=i P^{j} \frac{\partial}{\partial H_{0}} F\left(H_{0}\right),
$$

where $F$ is some (analytic) function of $H_{0}$ only (see [64] for details). The difficult part is to show the simultaneous block diagonalization of the boost and Hamilton operators.

Starting from the Okubo block-diagonalization condition

$$
\lambda\left(H_{I}+\left[H_{0}, A\right]+\left[H_{I}, A\right]-A H_{I} A\right) \eta=0,
$$

we have to show that the block-diagonalization condition is also valid for the boost operator:

$$
\lambda\left(K_{I}^{j}+\left[K_{0}^{j}, A\right]+\left[K_{I}^{j}, A\right]-A K_{I}^{j} A\right) \eta=0 .
$$

Our proof makes use of a perturbative expansion. For this reason, we rescale all couplings $c_{j}$ in a given Hamiltonian by

$$
c_{j} \rightarrow g c_{j}
$$

and write the operator $A$ in terms of the expansion in powers of the universal coupling constant $g$ via

$$
A=\sum_{n=1}^{\infty} g^{n} A_{n} .
$$

At leading order in $g$, we get

$$
A_{1} \eta|\beta\rangle=\frac{1}{E_{\beta}-H_{0}} \lambda H_{I} \eta|\beta\rangle \quad \text { and } \quad\langle\alpha| \lambda A_{1}=\langle\alpha| \lambda H_{I} \eta \frac{1}{H_{0}-E_{\alpha}},
$$

where we assume that the states $|\alpha\rangle$ and $|\beta\rangle$ are eigenstates of the free Hamiltonian,

$$
H_{0}|\alpha\rangle=E_{\alpha}|\alpha\rangle \text { and } H_{0}|\beta\rangle=E_{\beta}|\beta\rangle .
$$

In the following, we will often make use of the relation

$$
\left\langle\alpha\left|\lambda\left(K_{0}^{j} \frac{1}{E_{\beta}-H_{0}} X-X \frac{1}{H_{0}-E_{\alpha}} K_{0}^{j}\right) \eta\right| \beta\right\rangle=\frac{1}{E_{\beta}-E_{\alpha}}\left\langle\alpha\left|\lambda\left[K_{0}^{j}, X\right] \eta\right| \beta\right\rangle,
$$

where $X$ is some translationally invariant operator. We used here the last relation of Eq. (A.2) along with the translational invariance of the operators $H_{0}$ and $X$. With these relations we are ready to consider the commutator of the free boost with $A$ :

$$
\left\langle\alpha\left|\lambda\left[K_{0}^{j}, A_{1}\right] \eta\right| \beta\right\rangle=\frac{1}{E_{\beta}-E_{\alpha}}\left\langle\alpha\left|\lambda\left[K_{0}^{j}, H_{I}\right] \eta\right| \beta\right\rangle=\frac{1}{E_{\beta}-E_{\alpha}}\left\langle\alpha\left|\lambda\left[H_{0}, K_{I}^{j}\right] \eta\right| \beta\right\rangle=-\left\langle\alpha\left|\lambda K_{I}^{j} \eta\right| \beta\right\rangle,
$$


which is the desired relation of Eq. (A.4) to order $g$. So far, there was no difference to [64]. The difference starts to show up from order $g^{2}$. To verify Eq. (A.4) at order $g^{2}$, we start with Eq. (A.3). At order $g^{2}$, we get

$$
\lambda\left(\left[H_{0}, A_{2}\right]+\left[H_{I}, A_{1}\right]\right) \eta=0
$$

which is equivalent to

$$
A_{2} \eta|\beta\rangle=\frac{1}{E_{\beta}-H_{0}} \lambda\left[H_{I}, A_{1}\right] \eta|\beta\rangle \quad \text { and } \quad\langle\alpha| \lambda A_{2}=\langle\alpha| \lambda\left[H_{I}, A_{1}\right] \eta \frac{1}{H_{0}-E_{\alpha}} .
$$

Starting from these relations we consider the commutator

$$
\begin{aligned}
\left\langle\alpha\left|\lambda\left[K_{0}^{j}, A_{2}\right] \eta\right| \beta\right\rangle & =\frac{1}{E_{\beta}-E_{\alpha}}\left\langle\alpha\left|\lambda\left[K_{0}^{j},\left[H_{I}, A_{1}\right]\right] \eta\right| \beta\right\rangle \\
& =\frac{1}{E_{\beta}-E_{\alpha}}\left(\left\langle\alpha\left|\lambda\left[H_{I},\left[K_{0}^{j}, A_{1}\right]\right] \eta\right| \beta\right\rangle+\left\langle\alpha\left|\lambda\left[A_{1},\left[H_{I}, K_{0}^{j}\right]\right] \eta\right| \beta\right\rangle\right) .
\end{aligned}
$$

Using Eq. (A.10) and the second relation in Eq. (A.2), we get

$$
\begin{aligned}
\left\langle\alpha\left|\lambda\left[K_{0}^{j}, A_{2}\right] \eta\right| \beta\right\rangle & \left.=\frac{1}{E_{\beta}-E_{\alpha}}\left(\langle\alpha| \lambda\left[\lambda K_{I}^{j} \eta, H_{I}\right]\right] \eta|\beta\rangle+\left\langle\alpha\left|\lambda\left[A_{1},\left[K_{I}^{j}, H_{0}\right]\right] \eta\right| \beta\right\rangle\right) \\
& \left.=\frac{1}{E_{\beta}-E_{\alpha}}\left(\langle\alpha| \lambda\left[\lambda K_{I}^{j} \eta, H_{I}\right]\right] \eta|\beta\rangle+\left\langle\alpha\left|\lambda\left[K_{I}^{j},\left[A_{1}, H_{0}\right]\right] \eta\right| \beta\right\rangle+\left\langle\alpha\left|\lambda\left[H_{0},\left[K_{I}^{j}, A_{1}\right]\right] \eta\right| \beta\right\rangle\right) .
\end{aligned}
$$

Using Eq. (A.3), restricted to order $g$, we get

$$
\begin{aligned}
\left\langle\alpha\left|\lambda\left[K_{0}^{j}, A_{2}\right] \eta\right| \beta\right\rangle & \left.=\frac{1}{E_{\beta}-E_{\alpha}}\left(\langle\alpha| \lambda\left[\lambda K_{I}^{j} \eta, H_{I}\right]\right] \eta|\beta\rangle+\left\langle\alpha\left|\lambda\left[K_{I}^{j}, \lambda H_{I} \eta\right] \eta\right| \beta\right\rangle+\left\langle\alpha\left|\lambda\left[H_{0},\left[K_{I}^{j}, A_{1}\right]\right] \eta\right| \beta\right\rangle\right) \\
& \left.=\frac{1}{E_{\beta}-E_{\alpha}}\left(\langle\alpha| \lambda\left[K_{I}^{j}, H_{I}\right]\right] \eta|\beta\rangle+\left\langle\alpha\left|\lambda\left[H_{0},\left[K_{I}^{j}, A_{1}\right]\right] \eta\right| \beta\right\rangle\right) .
\end{aligned}
$$

Using now the third relation in Eq. (A.2) we finally get

$$
\left\langle\alpha\left|\lambda\left[K_{0}^{j}, A_{2}\right] \eta\right| \beta\right\rangle=-\left\langle\alpha\left|\lambda\left[K_{I}^{j}, A_{1}\right] \eta\right| \beta\right\rangle .
$$

Note that in Ref. [64], the authors assumed that $\eta H_{I} \eta=0$, which leads to a simplified version of Eq. (A.11) and Eq. (A.16) (see Eqs. (40) and (43) of [64]). It is also important to note that for the derivation of Eq. (A.16), the relation $\left[K_{I}^{j}, H_{I}\right]=0$ is essential.

For orders higher than $g^{3}$, Eq. (A.3) becomes

$$
\lambda\left(\left[H_{0}, A_{n+1}\right]+\left[H_{I}, A_{n}\right]-\sum_{\nu=1}^{n-1} A_{\nu} H_{I} A_{n-\nu}\right) \eta=0, \quad n \geq 2 .
$$

Starting from this relation we have to show the validity of the corresponding equation for the boost operator:

$$
\lambda\left(\left[K_{0}^{j}, A_{n+1}\right]+\left[K_{I}^{j}, A_{n}\right]-\sum_{\nu=1}^{n-1} A_{\nu} K_{I}^{j} A_{n-\nu}\right) \eta=0, \quad n \geq 2 .
$$

We will show this by induction. To start the proof by induction, we need to consider order- $g^{3}$ terms which corresponds to the case of $n=2$. We rewrite Eq. (A.17) for $n=2$ to

$$
A_{3} \eta|\beta\rangle=\frac{1}{E_{\beta}-H_{0}} \lambda X_{3} \eta|\beta\rangle \quad \text { and } \quad\langle\alpha| \lambda A_{3}=\langle\alpha| \lambda X_{3} \eta \frac{1}{H_{0}-E_{\alpha}}
$$

with

$$
X_{3}=\left[H_{I}, A_{2}\right]-A_{1} H_{I} A_{1}
$$


For the commutator of the free boost with $A_{3}$ we get

$$
\left\langle\alpha\left|\lambda\left[K_{0}^{j}, A_{3}\right] \eta\right| \beta\right\rangle=\frac{1}{E_{\beta}-E_{\alpha}}\left\langle\alpha\left|\lambda\left[K_{0}^{j}, X_{3}\right] \eta\right| \beta\right\rangle,
$$

where we used Eq. (A.9). For further simplification, we make use of the general commutation-relation identities valid for arbitrary operators $A, B, C, D$

$$
\begin{aligned}
{[A,[B, C]] } & =[B,[A, C]]+[C,[B, A]], \\
{[A, B C D] } & =[A, B] C D+B[A, C] D+B C[A, D],
\end{aligned}
$$

to get

$$
\lambda\left[K_{0}^{j}, X_{3}\right] \eta=\lambda\left(\left[H_{I},\left[K_{0}^{j}, A_{2}\right]\right]+\left[A_{2},\left[H_{I}, K_{0}^{j}\right]\right]-\left[K_{0}^{j}, A_{1}\right] H_{I} A_{1}-A_{1}\left[K_{0}^{j}, H_{I}\right] A_{1}-A_{1} H_{I}\left[K_{0}^{j}, A_{1}\right]\right) \eta .
$$

Using Eq. (A.16), Eq. (A.10) and the second relation in Eq. (A.2), we get

$$
\lambda\left[K_{0}^{j}, X_{3}\right] \eta=\lambda\left(-\left[H_{I}, \lambda\left[K_{I}^{j}, A_{1}\right] \eta\right]+\left[A_{2},\left[K_{I}^{j}, H_{0}\right]\right]+K_{I}^{j} \eta H_{I} A_{1}+A_{1}\left[K_{I}^{j}, H_{0}\right] A_{1}+A_{1} H_{I} \lambda K_{I}^{j}\right) \eta .
$$

Using Eq. (A.22) and Eq. (A.11), we get

$$
\left[A_{2},\left[K_{I}^{j}, H_{0}\right]\right]=\left[K_{I}^{j},\left[A_{2}, H_{0}\right]\right]+\left[H_{0},\left[K_{I}^{j}, A_{2}\right]\right]=\left[K_{I}^{j}, \lambda\left[H_{I}, A_{1}\right] \eta\right]+\left[H_{0},\left[K_{I}^{j}, A_{2}\right]\right] .
$$

Using Eq. (A.23) and the order-g restriction of Eq. (A.3), we obtain

$$
\begin{aligned}
A_{1}\left[K_{I}^{j}, H_{0}\right] A_{1} & =\left[A_{1} K_{I}^{j} A_{1}, H_{0}\right]+A_{1} K_{I}^{j}\left[H_{0}, A_{1}\right]+\left[H_{0}, A_{1}\right] K_{I}^{j} A_{1} \\
& =\left[A_{1} K_{I}^{j} A_{1}, H_{0}\right]-A_{1} K_{I}^{j} \lambda H_{I} \eta-\lambda H_{I} \eta K_{I}^{j} A_{1} .
\end{aligned}
$$

Putting Eq. (A.26) and Eq. (A.27) in Eq. (A.25) and rearranging the summands we get

$$
\lambda\left[K_{0}^{j}, X_{3}\right] \eta=\lambda\left(\left[H_{0},\left[K_{I}^{j}, A_{2}\right]\right]+\left[A_{1} K_{I}^{j} A_{1}, H_{0}\right]+\left[A_{1},\left[H_{I}, K_{I}^{j}\right]\right]\right),
$$

where the last term vanishes due to $\left[H_{I}, K_{I}^{j}\right]=0$. With this we finally get

$$
\left\langle\alpha\left|\lambda\left[K_{0}^{j}, A_{3}\right] \eta\right| \beta\right\rangle=-\left\langle\alpha\left|\lambda\left[K_{I}^{j}, A_{2}\right] \eta\right| \beta\right\rangle+\left\langle\alpha\left|\lambda A_{1} K_{I}^{j} A_{1} \eta\right| \beta\right\rangle .
$$

This completes the proof for starting the induction.

We now make an induction assumption, that Eq. (A.18) is valid for some arbitrary $n \geq 3$. In the induction step, we have to proof the same relation for $n+1$. As before, we start with the application of $A_{n+2}$ to the initial and final states

$$
A_{n+2} \eta|\beta\rangle=\frac{1}{E_{\beta}-H_{0}} \lambda X_{n+2} \eta|\beta\rangle \quad \text { and } \quad\langle\alpha| \lambda A_{n+2}=\langle\alpha| \lambda X_{n+2} \eta \frac{1}{H_{0}-E_{\alpha}}
$$

with

$$
X_{n+2}=\left[H_{I}, A_{n+1}\right]-\sum_{\nu=1}^{n} A_{\nu} H_{I} A_{n+1-\nu}
$$

For the matrix element of the free boost commutator with $A_{n+2}$, we get, as previously,

$$
\left\langle\alpha\left|\lambda\left[K_{0}^{j}, A_{n+2}\right] \eta\right| \beta\right\rangle=\frac{1}{E_{\beta}-E_{\alpha}}\left\langle\alpha\left|\lambda\left[K_{0}^{j}, X_{n+2}\right] \eta\right| \beta\right\rangle .
$$

We now use Eq. (A.22) to obtain

$$
\lambda\left[K_{0}^{j}, X_{n+2}\right] \eta=\lambda\left(\left[H_{I},\left[K_{0}^{j}, A_{n+1}\right]\right]+\left[A_{n+1},\left[H_{I}, K_{0}^{j}\right]\right]-\sum_{\nu=1}^{n}\left(\left[K_{0}^{j}, A_{\nu}\right] H_{I} A_{n+1-\nu}+A_{\nu}\left[K_{0}^{j}, H_{I}\right] A_{n+1-\nu}\right.\right.
$$




$$
\left.\left.+A_{\nu} H_{I}\left[K_{0}^{j}, A_{n+1-\nu}\right]\right)\right) \eta
$$

Using the second relation in Eq. (A.2), Eq. (A.22) and Eq. (A.23), we get

$$
\begin{aligned}
{\left[A_{n+1},\left[H_{I}, K_{0}^{j}\right]\right] } & =\left[K_{I}^{j},\left[A_{n+1}, H_{0}\right]\right]+\left[H_{0},\left[K_{I}^{j}, A_{n+1}\right]\right] \\
A_{\nu}\left[K_{0}^{j}, H_{I}\right] A_{n+1-\nu} & =\left[H_{0}, A_{\nu} K_{I}^{j} A_{n+1-\nu}\right]+\left[A_{\nu}, H_{0}\right] K_{I}^{j} A_{n+1-\nu}+A_{\nu} K_{I}^{j}\left[A_{n+1-\nu}, H_{0}\right] .
\end{aligned}
$$

Putting these relations back into Eq. (A.33), we obtain

$$
\lambda\left[K_{0}^{j}, X_{n+2}\right] \eta=\lambda\left(\left[H_{0},\left[K_{I}^{j}, A_{n+1}\right]\right]-\sum_{\nu=1}^{n}\left[H_{0}, A_{\nu} K_{I}^{j} A_{n+1-\nu}\right]+R_{1}+R_{2}\right) \eta
$$

with

$$
\begin{aligned}
& R_{1}=\left[H_{I},\left[K_{0}^{j}, A_{n+1}\right]\right]+\left[K_{I}^{j},\left[A_{n+1}, H_{0}\right]\right] \\
& R_{2}=-\sum_{\nu=1}^{n}\left(\left[K_{0}^{j}, A_{\nu}\right] H_{I} A_{n+1-\nu}+\left[A_{\nu}, H_{0}\right] K_{I}^{j} A_{n+1-\nu}+A_{n+1-\nu} K_{I}^{j}\left[A_{\nu}, H_{0}\right]+A_{n+1-\nu} H_{I}\left[K_{0}^{j}, A_{\nu}\right]\right) .
\end{aligned}
$$

In the following, we need to show that

$$
\lambda\left(R_{1}+R_{2}\right) \eta=0
$$

The sum in $R_{2}$ has been rearranged in order to consider separately the cases $\nu=1,2$ and the rest of the sum, where we can apply the induction assumption. After application of the induction assumption, we obtain a double-sum contribution to $R_{2}$, which, however, vanishes:

$$
\begin{aligned}
& \sum_{\nu=3}^{n} \sum_{\mu=1}^{\nu-2}\left(A_{\mu} K_{I}^{j} A_{\nu-1-\mu} H_{I} A_{n+1-\nu}-A_{\mu} H_{I} A_{\nu-1-\mu} K_{I}^{j} A_{n+1-\nu}-A_{n+1-\nu} K_{I}^{j} A_{\mu} H_{I} A_{\nu-1-\mu}\right. \\
& \left.+A_{n+1-\nu} H_{I} A_{\mu} K_{I}^{j} A_{\nu-1-\mu}\right)=0 .
\end{aligned}
$$

Due to the vanishing of the double sum, we can take the $\nu=2$ case into the rest sum, such that we need to consider only the $\nu=1$ and $\nu \geq 2$ cases separately. With this we get

$$
\begin{aligned}
R_{2} & =\lambda K_{I}^{j} \eta H_{I} A_{n}-\lambda H_{I} \eta K_{I}^{j} A_{n}-A_{n} K_{I}^{j} \lambda H_{I} \eta+A_{n} H_{I} \lambda K_{I}^{j} \eta \\
& +\sum_{\nu=1}^{n-1}\left(\lambda\left[K_{I}^{j}, A_{\nu}\right] \eta H_{I} A_{n-\nu}-\lambda\left[H_{I}, A_{\nu}\right] \eta K_{I}^{j} A_{n-\nu}-A_{n-\nu} K_{I}^{j} \lambda\left[H_{I}, A_{\nu}\right] \eta+A_{n-\nu} H_{I} \lambda\left[K_{I}^{j}, A_{\nu}\right] \eta\right)
\end{aligned}
$$

After applying the induction assumption to $R_{1}$, we get

$$
R_{1}=-\left[H_{I}, \lambda\left[K_{I}^{j}, A_{n}\right] \eta\right]+\left[K_{I}^{j}, \lambda\left[H_{I}, A_{n}\right] \eta\right]+\sum_{\nu=1}^{n-1}\left(\left[H_{I}, A_{\nu} K_{I}^{j} A_{n-\nu}\right]-\left[K_{I}^{j}, A_{\nu} H_{I} A_{n-\nu}\right]\right)
$$

Adding Eqs. (A.40) and (A.41) together, we obtain

$$
\lambda\left(R_{1}+R_{2}\right) \eta=\lambda\left[A_{n},\left[H_{I}, K_{I}^{j}\right]\right] \eta+\sum_{\nu=1}^{n-1} A_{\nu}\left[H_{I}, K_{I}^{j}\right] A_{n-\nu}=0
$$

For the last equation, we again used the third relation in Eq. (A.2). With this, we get for Eq. (A.32)

$$
\left\langle\alpha\left|\lambda\left[K_{0}^{j}, A_{n+2}\right] \eta\right| \beta\right\rangle=-\left\langle\alpha\left|\lambda\left[K_{I}^{j}, A_{n+1}\right] \eta\right| \beta\right\rangle+\sum_{\nu=1}^{n}\left\langle\alpha\left|\lambda A_{\nu} K_{I}^{j} A_{n+1-\nu} \eta\right| \beta\right\rangle .
$$

This completes the proof of the induction step. 
It is instructive to discuss the consequence of the simultaneous block diagonalization in the symmetric energy momentum tensor (Belinfante) notation. In this notation the boost operator has a simple form at $x_{0}=0$

$$
K^{j}=-\int d^{3} x x^{j} \Theta^{00}\left(x_{0}=0, \vec{x}\right)
$$

The Okubo block-diagonalization condition for the Hamiltonian, expressed in terms of symmetric energy momentum tensor, is given by

$$
\int d^{3} x\left\langle\alpha\left|\lambda\left(\Theta^{00}(x)-\left[A, \Theta^{00}(x)\right]-A \Theta^{00}(x) A\right) \eta\right| \beta\right\rangle=0
$$

Here we used the relation

$$
H_{s}=\int d^{3} x \Theta^{00}(x)
$$

Using translational invariance of the operator $A$, we can rewrite Eq. (A.45) to

$$
\int d^{3} x\left\langle\alpha\left|\exp (-i \vec{P} \cdot \vec{x}) \lambda\left(\Theta^{00}(0)-\left[A, \Theta^{00}(0)\right]-A \Theta^{00}(0) A\right) \eta \exp (i \vec{P} \cdot \vec{x})\right| \beta\right\rangle=0,
$$

which means that

$$
(2 \pi)^{3} \delta\left(\vec{P}_{\alpha}-\vec{P}_{\beta}\right)\left\langle\alpha\left|\lambda\left(\Theta^{00}(0)-\left[A, \Theta^{00}(0)\right]-A \Theta^{00}(0) A\right) \eta\right| \beta\right\rangle=0 .
$$

We follow that for $\vec{P}_{\alpha}=\vec{P}_{\beta}$, we have

$$
\left\langle\alpha\left|\lambda\left(\Theta^{00}(0)-\left[A, \Theta^{00}(0)\right]-A \Theta^{00}(0) A\right) \eta\right| \beta\right\rangle=0 .
$$

On the other hand, due to the simultaneous block diagonalization of the boost, we get also

$$
(2 \pi)^{3}\left(\frac{\partial}{\partial P_{\alpha}^{j}} \delta\left(\vec{P}_{\alpha}-\vec{P}_{\beta}\right)\right)\left\langle\alpha\left|\lambda\left(\Theta^{00}(0)-\left[A, \Theta^{00}(0)\right]-A \Theta^{00}(0) A\right) \eta\right| \beta\right\rangle=0 .
$$

Integrating this equation over $\vec{P}_{\alpha}$, we get at $\vec{P}_{\alpha}=\vec{P}_{\beta}$

$$
\frac{\partial}{\partial P_{\alpha}^{j}}\left\langle\alpha\left|\lambda\left(\Theta^{00}(0)-\left[A, \Theta^{00}(0)\right]-A \Theta^{00}(0) A\right) \eta\right| \beta\right\rangle=0 .
$$

So, if we would denote

$$
f\left(\vec{P}_{\alpha}\right)=\left\langle\alpha\left|\lambda\left(\Theta^{00}(0)-\left[A, \Theta^{00}(0)\right]-A \Theta^{00}(0) A\right) \eta\right| \beta\right\rangle
$$

the simultaneous block diagonalization means that the function $f\left(\vec{P}_{\alpha}\right)$ is not only zero at $\vec{P}_{\alpha}=\vec{P}_{\beta}$ but has also an extremum at this momentum:

$$
f\left(\vec{P}_{\alpha}=\vec{P}_{\beta}\right)=\left.\frac{\partial}{\partial P_{\alpha}^{j}} f\left(\vec{P}_{\alpha}\right)\right|_{\vec{P}_{\alpha}=\vec{P}_{\beta}}=0, \quad j=1,2,3 .
$$

\section{Appendix B: Additional unitary transformations}

At leading order in the chiral expansion, there is one possibility for an additional unitary transformation

$$
\exp \left(S_{16}^{a x, \mathrm{LO}}-\text { h.c. }\right)=1+S_{16}^{a x, \mathrm{LO}}-\text { h.c. }+\mathcal{O}\left(\left(S_{16}^{a x, \mathrm{LO}}\right)^{2}\right)
$$

with

$$
S_{16}^{a x, \mathrm{LO}}=\alpha_{16}^{a x, \mathrm{LO}} \eta A_{0,1}^{(-1)} \lambda^{1} \frac{1}{E_{\pi}^{2}} H_{2,1}^{(1)} \eta
$$


Here $H_{a, b}^{(\kappa)}$ denotes an interaction from the Hamiltonian with $a$ nucleon and $b$ pion fields with the index $\kappa$ specified in Eq. (2.10). The kinetic energy operator in this notation is denoted by $H_{2,0}^{(2)}$. Further, $A_{a, b}^{(\kappa)}$ denotes an interaction from the Hamiltonian with one axial vector current, $a$ nucleon and $b$ pion fields.

At $\mathrm{N}^{2} \mathrm{LO}$, the following unitary transformations are possible:

$$
\exp \left(\sum_{i=1}^{30} S_{i}^{a x}-\text { h.c. }\right)=1+\sum_{i=1}^{30} S_{i}^{a x}-\text { h.c. }+\mathcal{O}\left(\left(S_{i}^{a x}\right)^{2}\right)
$$

where

$$
\begin{aligned}
& S_{1}^{a x}=\alpha_{1}^{a x} \eta A_{2,0}^{(0)} \eta H_{2,1}^{(1)} \lambda^{1} \frac{1}{E_{\pi}^{3}} H_{2,1}^{(1)} \eta, \\
& S_{2}^{a x}=\alpha_{2}^{a x} \eta H_{2,1}^{(1)} \lambda^{1} \frac{1}{E_{\pi}^{2}} A_{2,0}^{(0)} \lambda^{1} \frac{1}{E_{\pi}} H_{2,1}^{(1)} \eta, \\
& S_{3}^{a x}=\alpha_{3}^{a x} \eta H_{2,1}^{(1)} \lambda^{1} \frac{1}{E_{\pi}^{2}} A_{2,1}^{(1)} \eta, \\
& S_{4}^{a x}=\alpha_{4}^{a x} \eta H_{2,1}^{(1)} \lambda^{1} \frac{1}{E_{\pi}^{2}} A_{0,1}^{(-1)} \lambda^{2} \frac{1}{E_{\pi}} H_{2,1}^{(1)} \lambda^{1} \frac{1}{E_{\pi}} H_{2,1}^{(1)} \eta, \\
& S_{5}^{a x}=\alpha_{5}^{a x} \eta H_{2,1}^{(1)} \lambda^{1} \frac{1}{E_{\pi}} A_{0,1}^{(-1)} \lambda^{2} \frac{1}{E_{\pi}^{2}} H_{2,1}^{(1)} \lambda^{1} \frac{1}{E_{\pi}} H_{2,1}^{(1)} \eta, \\
& S_{6}^{a x}=\alpha_{6}^{a x} \eta H_{2,1}^{(1)} \lambda^{1} \frac{1}{E_{\pi}} A_{0,1}^{(-1)} \lambda^{2} \frac{1}{E_{\pi}} H_{2,1}^{(1)} \lambda^{1} \frac{1}{E_{\pi}^{2}} H_{2,1}^{(1)} \eta, \\
& S_{7}^{a x}=\alpha_{7}^{a x} \eta A_{0,1}^{(-1)} \lambda^{1} \frac{1}{E_{\pi}} H_{2,1}^{(1)} \lambda^{2} \frac{1}{E_{\pi}} H_{2,1}^{(1)} \lambda^{1} \frac{1}{E_{\pi}^{2}} H_{2,1}^{(1)} \eta, \\
& S_{8}^{a x}=\alpha_{8}^{a x} \eta A_{0,1}^{(-1)} \lambda^{1} \frac{1}{E_{\pi}} H_{2,1}^{(1)} \lambda^{2} \frac{1}{E_{\pi}^{2}} H_{2,1}^{(1)} \lambda^{1} \frac{1}{E_{\pi}} H_{2,1}^{(1)} \eta, \\
& S_{9}^{a x}=\alpha_{9}^{a x} \eta A_{0,1}^{(-1)} \lambda^{1} \frac{1}{E_{\pi}^{2}} H_{2,1}^{(1)} \lambda^{2} \frac{1}{E_{\pi}} H_{2,1}^{(1)} \lambda^{1} \frac{1}{E_{\pi}} H_{2,1}^{(1)} \eta, \\
& S_{10}^{a x}=\alpha_{10}^{a x} \eta H_{2,1}^{(1)} \lambda^{1} \frac{1}{E_{\pi}^{3}} A_{0,1}^{(-1)} \eta H_{2,1}^{(1)} \lambda^{1} \frac{1}{E_{\pi}} H_{2,1}^{(1)} \eta, \\
& S_{11}^{a x}=\alpha_{11}^{a x} \eta H_{2,1}^{(1)} \lambda^{1} \frac{1}{E_{\pi}^{2}} A_{0,1}^{(-1)} \eta H_{2,1}^{(1)} \lambda^{1} \frac{1}{E_{\pi}^{2}} H_{2,1}^{(1)} \eta, \\
& S_{12}^{a x}=\alpha_{12}^{a x} \eta H_{2,1}^{(1)} \lambda^{1} \frac{1}{E_{\pi}} A_{0,1}^{(-1)} \eta H_{2,1}^{(1)} \lambda^{1} \frac{1}{E_{\pi}^{3}} H_{2,1}^{(1)} \eta, \\
& S_{13}^{a x}=\alpha_{13}^{a x} \eta A_{0,1}^{(-1)} \lambda^{1} \frac{1}{E_{\pi}^{3}} H_{2,1}^{(1)} \eta H_{2,1}^{(1)} \lambda^{1} \frac{1}{E_{\pi}} H_{2,1}^{(1)} \eta, \\
& S_{14}^{a x}=\alpha_{14}^{a x} \eta A_{0,1}^{(-1)} \lambda^{1} \frac{1}{E_{\pi}^{2}} H_{2,1}^{(1)} \eta H_{2,1}^{(1)} \lambda^{1} \frac{1}{E_{\pi}^{2}} H_{2,1}^{(1)} \eta, \\
& S_{15}^{a x}=\alpha_{15}^{a x} \eta A_{0,1}^{(-1)} \lambda^{1} \frac{1}{E_{\pi}} H_{2,1}^{(1)} \eta H_{2,1}^{(1)} \lambda^{1} \frac{1}{E_{\pi}^{3}} H_{2,1}^{(1)} \eta, \\
& S_{16}^{a x}=\alpha_{16}^{a x} \eta A_{0,1}^{(-1)} \lambda^{1} \frac{1}{E_{\pi}^{2}} H_{2,1}^{(3)} \eta, \\
& S_{17}^{a x}=\alpha_{17}^{a x} \eta A_{0,1}^{(-1)} \lambda^{1} \frac{1}{E_{\pi}^{2}} H_{2,0}^{(2)} \lambda^{1} \frac{1}{E_{\pi}} H_{2,1}^{(1)} \eta, \\
& S_{18}^{a x}=\alpha_{18}^{a x} \eta A_{0,1}^{(-1)} \lambda^{1} \frac{1}{E_{\pi}} H_{2,0}^{(2)} \lambda^{1} \frac{1}{E_{\pi}^{2}} H_{2,1}^{(1)} \eta, \\
& S_{19}^{a x}=\alpha_{19}^{a x} \eta H_{2,0}^{(2)} \eta A_{0,1}^{(-1)} \lambda^{1} \frac{1}{E_{\pi}^{3}} H_{2,1}^{(1)} \eta, \\
& S_{20}^{a x}=\alpha_{20}^{a x} \eta A_{0,1}^{(-1)} \lambda^{1} \frac{1}{E_{\pi}^{3}} H_{2,1}^{(1)} \eta H_{2,0}^{(2)} \eta, \\
& S_{21}^{a x}=\alpha_{21}^{a x} \eta A_{0,1}^{(-1)} \lambda^{1} \frac{1}{E_{\pi}^{2}} H_{4,0}^{(2)} \lambda^{1} \frac{1}{E_{\pi}} H_{2,1}^{(1)} \eta,
\end{aligned}
$$




$$
\begin{aligned}
S_{22}^{a x} & =\alpha_{22}^{a x} \eta A_{0,1}^{(-1)} \lambda^{1} \frac{1}{E_{\pi}} H_{4,0}^{(2)} \lambda^{1} \frac{1}{E_{\pi}^{2}} H_{2,1}^{(1)} \eta, \\
S_{23}^{a x} & =\alpha_{23}^{a x} \eta H_{4,0}^{(2)} \eta A_{0,1}^{(-1)} \lambda^{1} \frac{1}{E_{\pi}^{3}} H_{2,1}^{(1)} \eta, \\
S_{24}^{a x} & =\alpha_{24}^{a x} \eta A_{0,1}^{(-1)} \lambda^{1} \frac{1}{E_{\pi}^{3}} H_{2,1}^{(1)} \eta H_{4,0}^{(2)} \eta, \\
S_{25}^{a x} & =\alpha_{25}^{a x} \eta A_{0,1}^{(-1)} \lambda^{1} \frac{1}{E_{\pi}^{2}} H_{2,2}^{(2)} \lambda^{1} \frac{1}{E_{\pi}} H_{2,1}^{(1)} \eta, \\
S_{26}^{a x} & =\alpha_{26}^{a x} \eta A_{0,1}^{(-1)} \lambda^{1} \frac{1}{E_{\pi}} H_{2,2}^{(2)} \lambda^{1} \frac{1}{E_{\pi}^{2}} H_{2,1}^{(1)} \eta, \\
S_{27}^{a x} & =\alpha_{27}^{a x} \eta A_{0,1}^{(-1)} \lambda^{1} \frac{1}{E_{\pi}^{2}} H_{2,1}^{(1)} \lambda^{2} \frac{1}{E_{\pi}} H_{2,2}^{(2)} \eta, \\
S_{28}^{a x} & =\alpha_{28}^{a x} \eta A_{0,1}^{(-1)} \lambda^{1} \frac{1}{E_{\pi}} H_{2,1}^{(1)} \lambda^{2} \frac{1}{E_{\pi}^{2}} H_{2,2}^{(2)} \eta, \\
S_{29}^{a x} & =\alpha_{29}^{a x} \eta H_{2,1}^{(1)} \lambda^{1} \frac{1}{E_{\pi}} A_{0,1}^{(-1)} \lambda^{2} \frac{1}{E_{\pi}^{2}} H_{2,2}^{(2)} \eta, \\
S_{30}^{a x} & =\alpha_{30}^{a x} \eta H_{2,1}^{(1)} \lambda^{1} \frac{1}{E_{\pi}^{2}} A_{0,1}^{(-1)} \lambda^{2} \frac{1}{E_{\pi}} H_{2,2}^{(2)} \eta .
\end{aligned}
$$

The operator $S_{16}^{a x}$ can have three possible contributions: the first one is due to the interaction with pion-nucleon vertex from $\mathcal{L}_{\pi N}^{(3)}$ in the static limit, the second one is due to the interaction with the $1 / m$ correction to the leading order pion-nucleon vertex and the third one is due to the interaction with a tadpole. For these three possibilities, we have different unitary phases. For this reason, we introduce instead of a single parameter $\alpha_{16}^{a x}$ three different phases, which we denote by

$$
\alpha_{16}^{a x, \text { Static }}, \quad \alpha_{16}^{a x, 1 / m}, \quad \alpha_{16}^{a x, \text { Tadpole }} .
$$

We also considered all possible unitary transformations of the form

$$
\exp \left(i\left(\sum_{i} \tilde{\alpha}_{i} \tilde{S}_{i}^{a x}+\text { h.c. }\right)\right)
$$

where the operators $\tilde{S}_{i}^{a x}$ are again given by a sequence of vertices from the Hamiltonian and energy denominators. These transformations, however, lead to non-factorizable effective interactions. For this reason, we set all the phases of these transformations to zero. At the considered order, one exception is given by the unitary transformation considered in Appendix C, whose generator does not involve pion fields.

Last but not least, one may consider unitary transformations, whose generators involve a time derivative acting on the external axial source. At fourth order in the chiral expansion, such UTs would generate static contributions to the single-nucleon axial charge and current operators $\propto k_{0}^{2}$. We found, however, that such UTs lead to non-factorizable operators unless all corresponding phases are set to zero.

\section{Appendix C: Vertices with time-derivatives of the axial-vector source}

In this appendix we provide some details concerning our treatment of the $\bar{d}_{22}$-vertex which involves a time derivative of the axial-vector source

$$
-d_{22} N^{\dagger} S_{\mu}\left(\partial^{2} \boldsymbol{a}^{\mu}-\partial^{\mu} \partial_{\nu} \boldsymbol{a}^{\nu}\right) \cdot \boldsymbol{\tau} N=d_{22} N^{\dagger} S_{\mu}\left(\partial^{\mu} \dot{\boldsymbol{a}}^{0}\right) \cdot \boldsymbol{\tau} N+\ldots
$$

In the formulation presented so far, all contributions depending on the energy transfer were generated solely from time derivatives of the additional unitary transformations involving external axial-vector sources. Indeed, we are free to perform partial time-integration in the action and eliminate this kind of terms in favor of time-derivatives of the nucleon fields. In the next step, we can eliminate time derivatives of the nucleon fields in the effective Lagrangian 
by applying the equation of motion. After these two steps, there cannot be any $k_{0}$-dependent contributions to the nuclear axial charge operators stemming from the $\bar{d}_{22}$ vertex. However, we are still free to apply an additional unitary transformation of the form

$$
\exp \left(i \beta_{1}^{a x} \bar{d}_{22} N^{\dagger} S_{\mu}\left(\partial^{\mu} \boldsymbol{a}^{0}\right) \cdot \boldsymbol{\tau} N\right)
$$

on the effective Hamiltonian. Here, $\beta_{1}^{a x}$ is an arbitrary dimensionless parameter. Due to its explicit time dependence through the appearance of the external source $\boldsymbol{a}^{0}$, this unitary transformation induces a $k_{0}$-dependent contribution to the single-nucleon charge operator of the form

$$
A_{1 \mathrm{~N}: \text { static, } \mathrm{UT}_{1}^{\prime}}^{0, a(Q)}=\beta_{1}^{a x} \bar{d}_{22} k_{0} \frac{\tau_{i}^{a}}{2} \vec{k} \cdot \vec{\sigma}_{i}
$$

In addition, when applied to the free Hamiltonian, it generates a relativistic correction to the single-nucleon charge operator

$$
A_{1 \mathrm{~N}: 1 / m, \mathrm{UT}_{2}}^{0, a(Q)}=\beta_{1}^{a x} \bar{d}_{22} \frac{\tau_{i}^{a}}{2} \vec{k} \cdot \vec{\sigma}_{i} \frac{p_{i}^{2}-p_{i}^{\prime 2}}{2 m}
$$

Further, when acting on the one-pion exchange and the leading contact interaction potential, it also induces the contributions to the $2 N$ charge operator of the kind

$$
\begin{aligned}
& A_{2 \mathrm{~N}: \text { static }, \mathrm{UT}_{3}}^{0, a(Q)}=\beta_{1}^{a x} \frac{\bar{d}_{22}}{2}\left[\tau_{1}^{a} \vec{k} \cdot \vec{\sigma}_{1}, V_{2 \mathrm{~N}: 1 \pi}^{\left(Q^{0}\right)}\right]+1 \leftrightarrow 2, \\
& A_{2 \mathrm{~N}: \text { static, } \mathrm{UT}_{4}}^{0, a(Q)}=\beta_{1}^{a x} \frac{\bar{d}_{22}}{2}\left[\tau_{1}^{a} \vec{k} \cdot \vec{\sigma}_{1}, V_{2 \mathrm{~N}: \mathrm{cont}}^{\left(Q^{0}\right)}\right]+1 \leftrightarrow 2 .
\end{aligned}
$$

On the other hand, it is instructive to trace back the contributions of the $\bar{d}_{22}$-vertex to the nuclear axial charge operator. To eliminate the dependence on the time derivative in the term

$$
-\bar{d}_{22} N^{\dagger} S_{\mu}\left(\partial^{\mu} \boldsymbol{a}^{0}\right) \cdot \boldsymbol{\tau} \dot{N}+\text { h.c. }
$$

we make a redefinition of the nucleon field via

$$
N \rightarrow N-i \bar{d}_{22} S_{\mu}\left(\partial^{\mu} \boldsymbol{a}^{0}\right) \cdot \boldsymbol{\tau} N
$$

This eliminates the term in Eq. (C.7) from the effective Lagrangian at the cost of introducing the new vertices

$$
\begin{aligned}
& i \frac{\bar{d}_{22}}{F_{\pi}} g_{A} N^{\dagger} S_{\mu}\left(\partial^{\mu} \boldsymbol{a}^{0}\right) \cdot \boldsymbol{\tau} S_{\nu}\left(\partial^{\nu} \boldsymbol{\pi}\right) \cdot \boldsymbol{\tau} N-i \bar{d}_{22} N^{\dagger} S_{\mu}\left(\partial^{\mu} \boldsymbol{a}^{0}\right) \cdot \boldsymbol{\tau} \frac{\nabla^{2}}{2 m} N \\
& +i C_{S} \bar{d}_{22} N^{\dagger} S_{\mu}\left(\partial^{\mu} \boldsymbol{a}^{0}\right) \cdot \boldsymbol{\tau} N N^{\dagger} N-4 i C_{T} \bar{d}_{22} N^{\dagger} S_{\mu}\left(\partial^{\mu} \boldsymbol{a}^{0}\right) \cdot \boldsymbol{\tau} S_{\nu} N N^{\dagger} S^{\nu} N+\text { h.c. }
\end{aligned}
$$

which generate contributions to the $1 \mathrm{~N}$ and $2 \mathrm{~N}$ charge operators. Adding the resulting $1 \mathrm{~N}$ terms to the one specified in Eq. (C.4), we finally get the corresponding relativistic correction:

$$
\left(1+\beta_{1}^{a x}\right) \bar{d}_{22} \frac{\tau_{i}^{a}}{2} \vec{k} \cdot \vec{\sigma}_{i} \frac{p_{i}^{2}-p_{i}^{\prime 2}}{2 m} .
$$

Further, adding the $2 \mathrm{~N}$ contributions to the static one-pion exchange and contact axial charge operator generated by the vertices in Eq. (C.9) to the terms in Eqs. (C.6) and (C.5) we obtain

$$
\left(1+\beta_{1}^{a x}\right) \frac{\bar{d}_{22}}{2}\left[\tau_{1}^{a} \vec{k} \cdot \vec{\sigma}_{1}, V_{2 \mathrm{~N}: 1 \pi}^{\left(Q^{0}\right)}+V_{2 \mathrm{~N}: \mathrm{cont}}^{\left(Q^{0}\right)}\right]+1 \leftrightarrow 2
$$

We see that choosing the unitary phase $\beta_{1}^{a x}$ according to $\beta_{1}^{a x}=-1$ results in the absence of contributions $\propto \bar{d}_{22}$ to the nuclear axial charge operator apart from the $1 \mathrm{~N}$ term

$$
-\bar{d}_{22} k_{0} \frac{\tau_{i}^{a}}{2} \vec{k} \cdot \vec{\sigma}_{i}
$$

Thus, for this particular choice, the expression for the single-nucleon charge operator agrees with the on-shell result. This is the choice of the unitary phase $\beta_{1}^{a x}$ we adopt in our derivation, see Eq. (4.14). It is conceivable that the choice 
$\beta_{1}^{a x}=-1$ is compatible with renormalizability of the $1 \mathrm{~N}$ current operator at the two-loop level which, however, goes beyond the scope of our work.

[1] S. Weinberg, Phys. Lett. B 251, 288 (1990).

[2] U.-G. Meißner, Nucl. Phys. A 737, 110 (2004) [nucl-th/0307091].

[3] E. Epelbaum, H. W. Hammer and U-G. Meißner, Rev. Mod. Phys. 81, 1773 (2009) [arXiv:0811.1338 [nucl-th]].

[4] R. Machleidt and D. R. Entem, Phys. Rept. 503, 1 (2011) [arXiv:1105.2919 [nucl-th]].

[5] E. Epelbaum, H. Krebs and U.-G. Meißner, Phys. Rev. Lett. 115, no. 12, 122301 (2015) [arXiv:1412.4623 [nucl-th]].

[6] D. R. Entem, N. Kaiser, R. Machleidt and Y. Nosyk, Phys. Rev. C 91, no. 1, 014002 (2015) [arXiv:1411.5335 [nucl-th]].

[7] D. R. Entem, N. Kaiser, R. Machleidt and Y. Nosyk, Phys. Rev. C 92, no. 6, 064001 (2015) [arXiv:1505.03562 [nucl-th]].

[8] U. van Kolck, Phys. Rev. C 49, 2932 (1994).

[9] E. Epelbaum, A. Nogga, W. Glöckle, H. Kamada, U.-G. Meißner and H. Witała, Phys. Rev. C 66, 064001 (2002) [nuclth/0208023].

[10] E. Epelbaum, Phys. Lett. B 639, 456 (2006) [nucl-th/0511025].

[11] S. Ishikawa and M. R. Robilotta, Phys. Rev. C 76, 014006 (2007) [arXiv:0704.0711 [nucl-th]].

[12] V. Bernard, E. Epelbaum, H. Krebs and U.-G. Meißner, Phys. Rev. C 77, 064004 (2008) [arXiv:0712.1967 [nucl-th]].

[13] V. Bernard, E. Epelbaum, H. Krebs and U.-G. Meißner, Phys. Rev. C 84, 054001 (2011) [arXiv:1108.3816 [nucl-th]].

[14] H. Krebs, A. Gasparyan and E. Epelbaum, Phys. Rev. C 85, 054006 (2012) [arXiv:1203.0067 [nucl-th]].

[15] H. Krebs, A. Gasparyan and E. Epelbaum, Phys. Rev. C 87, no. 5, 054007 (2013) [arXiv:1302.2872 [nucl-th]].

[16] E. Epelbaum, A. M. Gasparyan, H. Krebs and C. Schat, Eur. Phys. J. A 51, no. 3, 26 (2015) [arXiv:1411.3612 [nucl-th]].

[17] L. Girlanda, A. Kievsky and M. Viviani, Phys. Rev. C 84, 014001 (2011) [arXiv:1102.4799 [nucl-th]].

[18] H.-W. Hammer, A. Nogga and A. Schwenk, Rev. Mod. Phys. 85, 197 (2013) [arXiv:1210.4273 [nucl-th]].

[19] A. Ekström, B. D. Carlsson, K. A. Wendt, C. Forssen, M. Hjorth-Jensen, R. Machleidt and S. M. Wild, J. Phys. G 42, no. 3, 034003 (2015) [arXiv:1406.6895 [nucl-th]].

[20] R. J. Furnstahl, D. R. Phillips and S. Wesolowski, J. Phys. G 42, no. 3, 034028 (2015) [arXiv:1407.0657 [nucl-th]].

[21] E. Epelbaum, H. Krebs and U.-G. Meißner, Eur. Phys. J. A 51, no. 5, 53 (2015) [arXiv:1412.0142 [nucl-th]].

[22] S. Binder et al. [LENPIC Collaboration], Phys. Rev. C 93, no. 4, 044002 (2016) [arXiv:1505.07218 [nucl-th]].

[23] R. J. Furnstahl, N. Klco, D. R. Phillips and S. Wesolowski, Phys. Rev. C 92, no. 2, 024005 (2015) [arXiv:1506.01343 [nucl-th]].

[24] R. N. Perez, J. E. Amaro, E. Ruiz Arriola, P. Maris and J. P. Vary, Phys. Rev. C 92, no. 6, 064003 (2015) [arXiv:1510.02544 [nucl-th]].

[25] E. Epelbaum, PoS CD 15, 014 (2016) [arXiv:1510.07036 [nucl-th]].

[26] L. E. Marcucci, Few Body Syst. 55, 615 (2014) [arXiv:1311.0693 [nucl-th]].

[27] S. Bacca and S. Pastore, J. Phys. G 41, no. 12, 123002 (2014) [arXiv:1407.3490 [nucl-th]].

[28] T. S. Park, D. P. Min and M. Rho, Nucl. Phys. A 596, 515 (1996) [nucl-th/9505017].

[29] S. Pastore, R. Schiavilla and J. L. Goity, Phys. Rev. C 78, 064002 (2008) [arXiv:0810.1941 [nucl-th]].

[30] S. Pastore, L. Girlanda, R. Schiavilla, M. Viviani and R. B. Wiringa, Phys. Rev. C 80, 034004 (2009) [arXiv:0906.1800 [nucl-th]].

[31] S. Pastore, L. Girlanda, R. Schiavilla and M. Viviani, Phys. Rev. C 84, 024001 (2011) [arXiv:1106.4539 [nucl-th]].

[32] S. Kölling, E. Epelbaum, H. Krebs and U.-G. Meißner, Phys. Rev. C 80, 045502 (2009) [arXiv:0907.3437 [nucl-th]].

[33] S. Kölling, E. Epelbaum, H. Krebs and U.-G. Meißner, Phys. Rev. C 84, 054008 (2011) [arXiv:1107.0602 [nucl-th]].

[34] M. Taketani, S. Machida and S. Ohnuma, Prog. Theor. Phys. (Kyoto) 7, 45 (1952).

[35] S. Okubo, Prog. Theor. Phys. 12, 603 (1954).

[36] E. Epelbaum, Eur. Phys. J. A 34, 197 (2007) [arXiv:0710.4250 [nucl-th]].

[37] M. Piarulli, L. Girlanda, L. E. Marcucci, S. Pastore, R. Schiavilla and M. Viviani, Phys. Rev. C 87, no. 1, 014006 (2013) [arXiv:1212.1105 [nucl-th]].

[38] D. Rozpedzik, J. Golak, S. Kolling, E. Epelbaum, R. Skibiński, H. Witała and H. Krebs, Phys. Rev. C 83, 064004 (2011) [arXiv:1103.4062 [nucl-th]].

[39] S. Kölling, E. Epelbaum and D. R. Phillips, Phys. Rev. C 86, 047001 (2012) [arXiv:1209.0837 [nucl-th]].

[40] R. Skibiński et al., Phys. Rev. C 93, no. 6, 064002 (2016) [arXiv:1604.03395 [nucl-th]].

[41] T. S. Park, D. P. Min and M. Rho, Phys. Rept. 233, 341 (1993) [hep-ph/9301295].

[42] T. S. Park et al., Phys. Rev. C 67, 055206 (2003) [nucl-th/0208055].

[43] R. Lazauskas, Y. H. Song and T. S. Park, Phys. Rev. C 83, 034006 (2011) [arXiv:0905.3119 [nucl-th]].

[44] L. E. Marcucci, A. Kievsky, S. Rosati, R. Schiavilla and M. Viviani, Phys. Rev. Lett. 108, 052502 (2012) [arXiv:1109.5563 [nucl-th]].

[45] L. E. Marcucci, R. Schiavilla and M. Viviani, Phys. Rev. Lett. 110, no. 19, 192503 (2013) [arXiv:1303.3124 [nucl-th]].

[46] A. Ekström, G. R. Jansen, K. A. Wendt, G. Hagen, T. Papenbrock, S. Bacca, B. Carlsson and D. Gazit, Phys. Rev. Lett. 113, no. 26, 262504 (2014) [arXiv:1406.4696 [nucl-th]].

[47] D. Gazit, S. Quaglioni and P. Navratil, Phys. Rev. Lett. 103, 102502 (2009) [arXiv:0812.4444 [nucl-th]]. 
[48] C. Hanhart, U. van Kolck and G. A. Miller, Phys. Rev. Lett. 85, 2905 (2000) [nucl-th/0004033].

[49] V. Baru, E. Epelbaum, J. Haidenbauer, C. Hanhart, A. E. Kudryavtsev, V. Lensky and U.-G. Meißner, Phys. Rev. C 80, 044003 (2009) [arXiv:0907.3911 [nucl-th]].

[50] V. Lensky, V. Baru, E. Epelbaum, C. Hanhart, J. Haidenbauer, A. E. Kudryavtsev and U.-G. Meißner, Eur. Phys. J. A 33, 339 (2007) [arXiv:0704.0443 [nucl-th]].

[51] A. Gardestig and D. R. Phillips, Phys. Rev. C 73, 014002 (2006) [nucl-th/0501049].

[52] S. X. Nakamura, Phys. Rev. C 77, 054001 (2008) [arXiv:0709.1239 [nucl-th]].

[53] V. A. Andreev et al. [MuCap Collaboration], Phys. Rev. Lett. 99, 032002 (2007) [arXiv:0704.2072 [nucl-ex]].

[54] L. E. Marcucci, Int. J. Mod. Phys. A 27, 1230006 (2012) [arXiv:1112.0113 [nucl-th]].

[55] J. Adam, Jr., M. Tater, E. Truhlik, E. Epelbaum, R. Machleidt and P. Ricci, Phys. Lett. B 709, 93 (2012) [arXiv:1110.3183 [nucl-th]].

[56] A. Baroni, L. Girlanda, S. Pastore, R. Schiavilla and M. Viviani, Phys. Rev. C 93, no. 1, 015501 (2016) Erratum: [Phys. Rev. C 93, no. 4, 049902 (2016)] [arXiv:1509.07039 [nucl-th]].

[57] J. L. Friar, Phys. Rev. C 60, 034002 (1999) [nucl-th/9901082].

[58] J. Gasser, M. E. Sainio and A. Svarc, Nucl. Phys. B 307, 779 (1988).

[59] N. Fettes, U.-G. Meißner, M. Mojžiš and S. Steininger, Annals Phys. 283, 273 (2000) [Annals Phys. 288, 249 (2001)] [hep-ph/0001308].

[60] E. Epelbaum, arXiv:1001.3229 [nucl-th].

[61] J. Gasser and H. Leutwyler, Annals Phys. 158 (1984) 142.

[62] V. V. Kotlyar, Y. P. Melnik and A. V. Shebeko, Phys. Part. Nucl. 26, 79 (1995) [Fiz. Elem. Chast. Atom. Yadra 26, 192 (1995)].

[63] K. Ohta and M. Ichimura, Nucl. Phys. A 491 (1989) 509.

[64] A. Krüger and W. Glöckle, nucl-th/9712043.

[65] W. Glöckle and L. Müller, Phys. Rev. C 23 (1981) 1183.

[66] J. L. Friar and S. A. Coon, Phys. Rev. C 49, 1272 (1994).

[67] J. Gasser, M. A. Ivanov, E. Lipartia, M. Mojžiš and A. Rusetsky, Eur. Phys. J. C 26, 13 (2002) [hep-ph/0206068].

[68] V. Bernard, N. Kaiser and U.-G. Meißner, Nucl. Phys. A 611, 429 (1996) [hep-ph/9607428].

[69] H. W. Fearing, R. Lewis, N. Mobed and S. Scherer, Phys. Rev. D 56, 1783 (1997) [hep-ph/9702394].

[70] V. Bernard, N. Kaiser, T. S. H. Lee and U.-G. Meißner, Phys. Rept. 246, 315 (1994), [hep-ph/9310329].

[71] M. R. Schindler, T. Fuchs, J. Gegelia and S. Scherer, Phys. Rev. C 75, 025202 (2007) [nucl-th/0611083].

[72] N. Kaiser, Phys. Rev. C 67, 027002 (2003) [nucl-th/0301034].

[73] E. Epelbaum and U.-G. Meißner, Phys. Rev. C 72, 044001 (2005) [nucl-th/0502052].

[74] M. Hoferichter, P. Klos and A. Schwenk, Phys. Lett. B 746, 410 (2015) [arXiv:1503.04811 [hep-ph]].

[75] G. Shen, L. E. Marcucci, J. Carlson, S. Gandolfi and R. Schiavilla, Phys. Rev. C 86, 035503 (2012) [arXiv:1205.4337 [nucl-th]].

[76] A. Baroni, L. Girlanda, A. Kievsky, L. E. Marcucci, R. Schiavilla and M. Viviani, Phys. Rev. C 94, no. 2, 024003 (2016) [arXiv:1605.01620 [nucl-th]].

[77] E. Epelbaum, W. Glöckle and U.-G. Meißner, Nucl. Phys. A 747, 362 (2005) [nucl-th/0405048].

[78] J. Menendez, D. Gazit and A. Schwenk, Phys. Rev. Lett. 107, 062501 (2011) [arXiv:1103.3622 [nucl-th]]. 